\title{
RÉPERTOIRE SYSTÉMATIQUE
}

\section{DES NOMS DE GENRES DE VERTÉBRÉS}

\author{
(suite)
}

Par Yves J. GOLVAN

Osculatia BONAPARTE 1854: Av. COLUMBIF. Columbid. (Columbin.).

Oshimia JORDAN 1919 : P.-PERCIF. (Anabantoid.) Anabantid.

Osmerus F O R S K A L L 1775 : P.CLUPEIF. (Salmonoid.) Osmerid.

Osmotreron BONAPARTE 1854: Av. COLUMBIF. Columbid. (Treronin.).

Ospatulus HERRE 1924 : P.-CYPRINIF. (Cyprinoid.) Cyprinid.

Osphranter GOULD 1842 : M.-MARSUP. Macropodid. (Macropodin.).

Osphronemus de LACEPEDE 1802: P.-PERCIF. (Anabantoid.) Osphromenid.

Ossifraga HOMBRON et JACQUINOT 1844 nec WOOD 1835: syn. de Macronectes RICHEMOND 1905.

Osteobagrus JAYARAM $1953: s . / g$. de Mystrus.

Osteoblepharon SCHMIDT 1919 : syn. d'Osteolaemus COPE.

Osteobrama HECKEL 1843 : P.-CYPRINIF. (Cyprinoid.) Cyprinid.

Osteocheilichthys HORA 1942 : P.CYPRINIF. (Cyprinoid.) Cyprinid.
Osteochilus GUNTHER 1868 : syn. de Rohita CUVIER et VALENCIENNES 1842.

Osteochromis FRANZ 1910: P.PERCIF. (Percoid.) Chaetodontid.

Osteogaster COPE 1893 : P.-CYPRINIF. (Siluroid.) Callichthyid.

Osteogeneiosus BLEEKER 1846 : P.-CYPRINIF. (Siluroid.) Bagrid.

Osteoglossum VANDELLI 1797 : P. CLUPEIF. (Osteoglossoid.) Osteoglossid.

Osteolaemus COPE 1860 : R.-LORICAT. Crocodilid.

Osteomystax : P.-CYPRINIF. (Siluroid.) Doradid.

Osteosternum WU 1929: syn. de Ooeidozyga KUHL et von HAS. SELT.

Ostinops CABANIS 1851: Av, PASSERIF. (Acromyod.) Icterid. (Cacicin.).

Ostophycephalus OGILBY 1899 : P.-CYPRINIF. (Siluroid.) Plotosid.

Ostorhynchus SWAINSON 1839: P.-PERCIF. (Percoid.) Serranid.

Ostracion LIN NE 1758: P.-TE. TRAODONTIF. (Ostracionoid.) Ostracionid. 
Ostracoberyx FOWLER 1934 : P.BERYCIF. Berycid. (ou Ostracoberycid.).

Ostralegus Mc GILLAVRAY 1842 : syn. de Haematopus LINNE 1758.

Ostreoblennius WHITLEY 1930 : s./g. de Petroscirtes.

Ostreogobius WHITLEY 1930 : P.PERCIF. (Gobioid.) Gobiid.

Otakia JORDAN et SNYDER 1900 : P.-CYPRINIF. (Cyprinoid.) Cyprinid.

Otalis de BUEN 1945 : P.-MUGILIF. Atherinid.

Otaria PERON 1816 : M.-PINNIP. Otariid. (Otariin.).

Otelaphus FITZINGER 1874: syn. de Odocoileus RAFINESQUE 1832.

Othonias JORDAN et THOMPSON 1911: P.-PERCIF. (Percoid.) Sciaenid.

Othonocheirodus MYERS 1927 : P.CYPRINIF. (Characoid.) Characid.

Othonophanes EIGENMANN 1903 : P.CYPRINIF. (Characoid.) Characid.

Othonops SMITH 1881: P.-PERCIF. (Gobioid.) Gobiid.

Othos CASTELNAU 1875 : P.-PERCIF. (Percoid.) Serranid.

Othyphantes SHELLEY 1896: Av. PASSERIF. (Acromyod.) Ploceid.

Otidiphaps GOULD 1870 : Av. COLUMBIF. Columbid. (Columbin.).

Otis LINNE 1758: Av. RALLIF. otidid.

Otisorex DEKA Y 1842: syn. de Sorex LINNE 1735.

Otocinclus COPE 1871 : P.-CYPRINIF. (Siluroid.) Bagrid.

Otocolobus BRANDT 1841 : s./g. de Felis LINNE 1758.

Otocompsa CABANIS 1851: Av. PASSERIF. (Acromyod.) Pycnonotid.

(Otocorydopsis BIANCHI 1907 : Av. PASSERIF. (Acromyod.) Alaudid.

Otocorys BONAPARTE 1838 : syn. de Chionophilus BREHM.
Otocryptis W A GLER 1830 : R.SQUAMAT. (Saur.) Agamid.

Otocyon MULLER 1836 : M.-FISSIP. Canid. (Otocyonin.).

Otoes FISCHER 1817: composite, non valide, vide Callirhinus GRAY 1859.

Otogale GRAY 1863 : syn. de Euoticus.

Otogyps GRAY 1841: Av. FALCONIF. Falconid. (Aegypiin.).

Otohime JORDAN et STARKS 1907: P.-PERCIF. (Scorpaenoid.) Triglid.

Otolithoides FOWLER 1933 : P.PERCIF. (Percoid.) Sciaenid.

Otolithus CUVIER 1829: P.-PERCIF. (Percoid.) Sciaenid.

Otomela BONAPARTE 1853: Av. PASSERIF. (Acromyod.) Laniid.

Otomops THOMAS 1913 : M.-CHIROPT. (Microchiropt.) Molossid.

Otomys CUVIER 1823: M.-RODENT. (Myomorph.) Murid. (Otomyin.).

Otonycteris PETERS 1859 : M.-CHIROPT. (Microchiropt.) Vespertilionid. (Vespertilionin.).

Otonyctomys ANTHONY 1932 : M.RODENT. (Myomorph.) Cricetid. (Cricetin.).

Otoperca BOULENGER 1915 : P. PERCIF. (Percoid.) Lutjanid.

Otophanes BREWSTER 1888: Av. CAPRIMULGIF. Caprimulgid. (Caprimulgin.).

Otopharynx REGAN 1920 : P.-PERCIF. (Percoid.) Cichlid.

Otophidium (GILL) JORDAN 1887 : P.-PERCIF. (Ophidioid.) Ophidiid.

Otophryne BOULENGER 1900 : Amph. ANOUR. Microhylid.

Otopithecus TROUESSART 1897 : syn. de Leontocebus WAGNER 1839.

Otopterus LYDEKKER 1891 : syn. de Macrotus GRAY.

Otosaurus GRAY 1845 : R.-SQUAMAT. (Saur.) Scincid.

Otosciurus NELSON 1899 : s./g. de Sciurus LINNE 1758. 
Otospermophilus BRANDT 1844: s./g. de Citellus OKEN 1816.

Otothyris MYERS 1927 : P.-CYPRINIF. (Siluroid.) Loricariid.

Ototylomys MERRIAM 1901: M.RODENT. (Myomorph.) Cricetid. (Hesperomyin.).

Otrynter JORDAN et EVERMANN 1896 : P. - PERCIF. (Percoid.) Sparid.

Otus PENNANT 1769: Av. STRIGIF. Strigid. (Bubonin.).

Otyphantes : errat. pro Othyphantes SHELLEY 1896.

Oulodon von HAAST 1876 : syn. de Mesoplodon GERVAIS 1850.

Ourebia LAURILLARD 1841 : M.PARAX. (Solenodont.) Bovid. (Reduncin.).

Ovibos de BLAINVILLE 1816 : M.PARAX. (Solenodont.) Bovid. (Bovin.).

Ovis LINNE 1758 : M.-PARAX. (Solenodont.) Bovid. (Ovin.).

Ovoides CUVIER 1800 : P.-TETRAODONTIF. (Tetraodontoid.) Tetraodontid.

Owenavis MATHEWS 1912: Av. CUCULLIF. Cucullid.

Owsianka DYBOWSKY 1864 : P.CYPRINIF. (Cyprinoid.) Cyprinid.

Owstonia TANAKA 1908: P.-PERCIF. (Percoid.) Cepolid.

Oxuderces VALENCIENNES 1842 : P.-PERCIF. (Gobioid.) Gobiid.

Oxyanthias JORDAN et EVERMANN 1896 : P.-PERCIF. (Percoid.) Serranid.

Oxybarbus VAILLANT 1893: P.CYPRINIF. (Cyprinoid.) Cyprinid.

Oxybeles RICHARDSON 1846 : P.PERCIF. (Ophidioid.) Fierasferid.

Oxybelis WAGLER 1830 : R.-SQUAMAT. (Serpent.) Boigid.

Oxycephas RAFINESQUE 1810 : P.-GADIF. Macrurid.

Oxyceros HUME: Av. CORACIADIF. Bucerotid.

Oxychaetodon BLEEKER 1876 : P.PERCIF. (Percoid.) Chaetodontid.
Oxycheilinus GILL 1863 : P.-PERCIF. (Labroid.) Labrid.

Oxycirrhites BLEEKER 1857 : P.PERCIF. (Percoid.) Cirrhitid.

Oxyconger BLEEKER 1865: P.ANGUILLIF. (Congroid.) Congrid. (ou Muraenesocid.).

Oxycottus JORDAN et EVERMANN 1898 : P. - PERCIF. (Cottoid.) Cottid.

Oxydactyla van KAMPEN 1913 : syn. de Sphenophryne PETERS et DORIA.

Oxydoras KNERR 1855 : P.-CYPRINIF. (Siluroid.) Doradid.

Oxydozyga TSCHUDI 1838 : Amph. ANOUR. Ranid.

Oxyechus REICHENBACH 1852 : Av. CHARADRIIF. Charadriid. (Charadriin.).

Oxyeleotris BLEEKER 1874: P.PERCIF. (Gobioid.) Eleotrid.

Oxygadus GILBERT et $H U B B S$ 1920 : P.-GADIF. Macrurid.

Oxygaster van HASSELT 1823 : P.CYPRINIF. (Cyprinoid.) Cobitid.

Oxygeneum FORBES 1885 : P.-CYPRINIF. (Cyprinoid.) Cyprinid.

Oxyglanis VINCIGUERRA 1898 : P.-CYPRINIF. (Siluroid.) Loricariid.

Oxyglossus TSCHUDI 1838 : Amph. ANOUR. Ranid.

Oxygous HODGSON 1841: syn. de Canis LINNE 1758.

Oxyjulis GILL 1864 : P.-PERCIF. (Labroid.) Labrid.

Oxylabes SHARPE 1870 : Av. PASSERIF. (Acromyod.) Timaliid.

Oxylabrax B L E E K E R 1876 : P.PERCIF. (Percoid.) Serranid.

Oxylebius GILL 1863 : P.-PERCIF. (Scorpaenoid.) Hexagrammid.

Oxyloricaria BLEEKER 1863 : P.CYPRINIF. (Siluroid.) Loricariid.

Oxymacrurus BLEEKER 1874 : P.GADIF. Macrurid.

Oxymetopon BLEEKER 1860 : P.PERCIF. (Gobioid.) Eleotrid.

Oxymormyrus BLEEKER 1874 : P.CLUPEIF. (Mormyroid.) Mormyrid. 
Oxymugil WHITLEY 1948 : P.-MUGILIF. Mugilid.

Oxymycterus WATERHOUSE 1837 : M.-RODENT. (Myomorph.) Cricetid. (Hesperomyin.).

Oxynotus RAFINESQUE 1810 : P. SQUALIF. (Squaloid.) Squalid.

Oxyodon BRAUER 1906 : P.-PERCIF. (Percoid.) Serranid.

Oxypelia SALVADORI 1893: Av. COLUMBIF. Columbid. (Columbin.).

Oxypogon GOULD 1848 : Av. APODIF. Trochilid.

Oxyporhamphus GILL 1864: P.BELONIF. (Exocoetoid.) Exocoetid.

Oxyrhabdium BOULENGER 1892 : R.-SQUAMAT. (Serpent.) Colubrid.

Oxyrhamphus STRICHLAND 1841 : syn. d'Oxyrhynchus TEMMINCK.

Oxyrhin KAUP 1829 : syn. de Sorex LINNE 1735.

Oxyrhina AGASSIZ 1835 : P.-GALEIF. (Isuroid.) Isurid.

Oxyrhopus WAGLER 1830 : syn. de Pseudoboa SCHNEIDER.

Oxyrhynchus TEMMINCK 1822 : Av. PASSERIF. (Mesomyod.) Oxyrhynchid. (ou Tyrannid.).

Oxyropsis EIGENMANN 1889 : P.CYPRINIF. (Siluroid.) Loricariid.

Oxyruncus TEMMINCK 1820: errat. pro Oxyrhynchus TEMMINCK 1822 .

Oxystomus RAFINESQUE 1810 (nec FISCHER 1803): syn. de Bertinulus WHITLEY 1948.

Oxyura BONAPARTE 1831: Av. ANATIF. Anatid. (Anatin.).

Oxyuranus KINGHORN 1923 : R.SQUAMAT. (Serpent.) Elapid.

Oxyurichthys BLEEKER 1874: P.PERCIF. (Goboid.) Gobiid.

Oxyurus SWAINSON: syn. d'Aphrastura OBERHOLSER 1899.

Oxyurus RAFINESQUE 1810 : P.ANGUILLIF. (Congroid.) Congrid.

Ozanna REICHENBACH 1845 : syn.
d'Hippotragus SUNDEVALL 1844.

Ozotoceros AMEGHINO 1891 : M.PARAX. (Solenodont.) Cervid.

Pachycalamus GUNTHER 1881: R.-SQUAMAT. (Saur.) Tejid.

Pachycara ZUGMAYER 1911: P.PERCIF. (Blennioid.) Zoarcid.

Pachycare GOULD 1876: Av. PASSERIF. (Acromyod.) Pachycephalid.

Pachycarichthys WHITLEY 1931 : syn. de Pachycara ZUGMAYER 1911.

Pachycephala VIGORS 1825: Av. PASSERIF. (Acromyod.) Pachycephalid.

Pachycephalixus BUTTIKOFER 1896: Av. PASSERIF. (Acromyod.) Pachycephalid.

Pachycephalopsis $S A L V A D O R I$ 1881: Av. PASSERIF. (Acromyod.) Pachycephalid.

Pachyceros GROMOVA $1936: \mathrm{s} . / \mathrm{g}$. de Ovis LINNE 1758.

Pachychilon STEINDACHNER 1882: P. - CYPRINIF. (Cyprinoid.) Cyprinid.

Pachycilodryas MATHEWS 1925 : Ãv. PASSERIF. (Acromyod.) Muscicapid.

Pachycoccyx CABANIS 1882: Av. CUCULLIF. Cucullid.

Pachydactylus WIEGMANN 1834: R.SQUAMAT. (Saur.) Geckonid.

Pachylabrus GIBBONS 1854: P. PERCIF. (Labroid.) Embiotocid.

Pachymetopon GUNTHER 1859: P.-PERCIF. (Percoid.) Sparid.

Pachynus REICHENOW 1881: Av. PSITTACIF. Psittacid.

Pachyomus GRAY : syn. d'Eptesicus RAFINESQUE 1820.

Pachyophis WERNER 1924: syn. de Trachyophis WERNER 1926.

Pachyophis NOPCSA 1923: R.SQUAMAT. (Serpent.) Colubrid.

Pachyotus GRA Y 1831: syn. de Scotophilus LEACH 1821. 
Pachypalminus THOMPSON 1912 : Amph. UROD. Ambystomid.

Pachypanchax MYERS 1933: P. CYPRINODONTIF. Cyprinodontid.

Pachyphantes SHELLEY 1896: Av. PASSERIF. (Acromyod.) Ploceid. (Plocein.).

Pachypops GILL 1861 : P.-PERCIF. (Percoid.) Sciaenid.

Pachypterus SWAINSON 1839 : P.CYPRINIF. (Siluroid.) Schilbeid.

Pachyptila IL L I G E R 1811: Av. PROCELLARIIF. Procellariid.

Pachypus SPIX: Amph. ANOUR. Leptodactylid.

Pachyrhamphus GRAY 1839: Av. PASSERIF. (Mesomyod.) Cotingid.

Pachyrhynchus SPIX 1825: Av. PASSERIF. (Mesomyod.) Cotingid.

Pachysoma GEOFFROY-ST-HILAIRE 1828 : syn. de Cynopterus CUVIER 1824.

Pachystila : errat. pro Pachyptila ILLIGER 1811.

Pachystomias GUNTHER 1887 : P.CLUPEIF. (Stomiatoid.) Melanostomiatoid.

Pachystomus HECKEL 1843 : P.CYPRINIF. (Cyprinoid.) Cyprinid.

Pachysylvia BONAPARTE 1850 : Av. PASSERIF. (Acromyod.) Vireonid.

Pachytila : errat. pro Pachyptila ILLIGER 1811.

Pachytriton BOULENGER 1879 : Amph. UROD. Salamnadrid.

Pachyula OGILBY 1898 : P.-CYPRINIF. (Siluroid.) Bagrid.

Pachyura SELYS-LONGCHAMPS 1839 : syn. de Suncus EHRENBERG 1833.

Pachyuromys LATASTE 1880 : M.RODENT. (Myomorph.) Gerbillid.

Pachyurus SWAINSON 1839: P.ANGUILLIF. (Anguilloid.) Moringuid.

Pachyurus AGASSIZ 1831 : P.-PERCIF. (Percoid.) Sciaenid.
Pacificolarus von BOETTICHER 1935: s./g. de Larus LINNE 1758.

Pactia KISHIDA et MORI 1931 : nom. nud. pro Myotis KAUP 1829.

Pacu SPIX 1829: P.-CYPRINIF, (Characoid.) Characid.

Padda REICHENBACH 1850: Av. PASSERIF. (Acromyod.) Ploceid. (Estrildin.).

Padogobius BERG 1932 : P.-PERCIF. (Gobioid.) Gobiid.

Paecilonetta EYTON 1838: errat. pro Poecilonitta EYTON 1838.

Paeneapocryptes HERRE 1927: s./g. d'Apocryptes CUVIER et VALENCIENNES.

Pagellus CUVIER et VALENCIENNES 1830 : P.-PERCIF. (Percoid.) Sparid.

Pagetopsis REGAN 1913 : P.-PERCIF. (Trachinoid.) Trachinid.

Pagoa MATHEWS 1913: syn. de Charadrius LINNE 1758.

Pagodroma BONAPARTE 1855 : Av. PROCELLARIIF. Procellariid.

Pagomys GRAY 1864: syn. de Phoca LINNE 1758.

Pagophila KAUP 1829: Av. LARIF. Larid. (Larin.).

Pagophilus GRAY 1844: syn. de Phoca LINNE 1758.

Pagophoca TROUESSART 1904: s./g. de Phoca LINNE 1758.

Pagothenia NICHOLS et LAMONTE 1936: P.-PERCIF. (Trachinoid.) Nototheniid.

Pagrichthys BLEEKER 1860 : P.PERCIF. (Percoid.) Sparid.

Pagrosomus GILL 1839 : P.-PERCIF. (Percoid.) Sparid.

Pagrus CUVIER 1817 : P.-PERCIF. (Percoid.) Sparid.

Paguma GRAY 1831: M.-FISSIP. Viverrid. (Viverrin.).

Paikea OLIVER 1922 : syn. de Mesoplodon GERVAIS 1850.

Painterius OBERHOLSER 1930 : Av. PASSERIF. (Acromyod.) Sturnid. (Graculin.).

Palaeaspis GRAY 1870 (nec CLAYPOLE 1885): R. -TESTUDIN. (Thecoph.) Pelomedusid. 
Palaenichthys KAUP 1872 : syn. de Lepidotrigla GUNTHER.

Palaeornis VIGORS 1825: Av. PSITTACIF. Psittacid.

Palamedaea LINNE 1766: syn. d'Anhima.

Palamita BONAPARTE 1831: P.PERCIF. (Scombroid.) Thunnid.

Paleosuchus GRAY 1862 : R.-LORICAT. Alligatorid.

Palinurichthys BLEEKER 1859: P. - PERCIF. (Stromateoid.) Stromateid.

Palinurus de KAY 1842 : P.-PERCIF. (Stromateoid.) Stromateid.

Pallasia NARDO 1840 : P.-PERCIF. (Cottoid.) Agonid.

Pallasina JORDAN et STARKS 1895: P.-PERCIF. (Cottoid.) Agonid.

Pallasiomys HEPTNER $1933: s . / g$. de Meriones ILLIGER 1811.

Pallidogobius HERRE 1953: P.PERCIF. (Gobioid.) Gobiid.

Palmatogecko ANDERSSON 1908 : R.-SQUAMAT. (Saur.) Geckonid.

Palmatorappia AHL 1927 : Amph. ANOUR. Hylid.

Palmatus LYDEKKER 1898: syn. de Dama FRISCH 1775.

Palmeria ROTSCHILD 1893: Av. PASSERIF. (Acromyod.) Drepanidid.

Palmichthys de BUEN $1946: s . / g$. de Chirostoma.

Paloa HERRE 1927 : P.-PERCIF. (Gobioid.) Eleotrid.

Palometa JORDAN et EVERMANN 1896 : P. - PERCIF. (Stromateoid.) Stromateid.

Palsartia WHITLEY 1943 : P.PERCIF. (Percoid.) Serranid.

Paludicola WAGLER 1830: Amph. ANOUR. Leptodactylid.

Paludipasser NEA VE 1909: Av. PASSERIF. (Acromyod.) Ploceid. (Passerin.).

Palumboena BONAPARTE 1854: Av. COLUMBIF. Columbid. (Columbin.).

Palumbus MOEHRING 1758: syn. de Columba LINNE 1758.
Palunolepis BARNARD 1927 : P.PERCIF. (Percoid.) Cheilodactylid.

Pama FOWLER 1933 : P.-PERCIF. (Percoid.) Sciaenid.

Pammeles GUNTHER 1860 : P.PERCIF. (Percoid.) Carangid.

Pampa REICHENBACH 1854: Av. APODIF. Trochilid.

Pampanoa FO WLER 1906 : P.PERCIF. (Percoid.) Carangid.

Pampatestudo LINDHOLM 1929: s./g. de Testudo LINNE 1758.

Pamphoria REGAN 1913 : P.-CYPRINODONTIF. Poeciliid.

Pamphorichthys REGAN 1913 : P.CYPRINODONTIF. Poeciliid.

Pampus BONAPARTE 1841: P.PERCIF. (Stromateoid.) Stromateid.

Pan OKEN 1816: M.-PRIMAT. (Simioid.) Pongid.

Panamichthys HUBBS 1925 : P.CYPRINODONTIF. Poeciliid.

Panaque EIGENMANN 1889: P.CYPRINIF. (Siluroid.) Loricariid.

Panchax CUVIER et VALENCIENNES 1846 : P.-CYPRINODONTIF. Cyprinodontid.

Pandaka HERRE 1927 : P.-PERCIF. (Gobioid.) Eleotrid.

Pandarctos GERVAIS 1870 : syn. d'Ailuropoda MILNEEDWARDS 1870.

Pandion SAVIGNY 1809 : Av. FALCONIF. Falconid. (Pandionin.).

Pangasianodon CHEVEY 1930 : P.CYPRINIF. (Siluroid.) Schilbeid. ou Silurid.

Pangasius CUVIER et VALENCIENNES 1840 : P.-CYPRINIF. (Siluroid.) Schilbeid.

Pangio BLYTH 1860 : P.-CYPRINIF. (Cyprinoid.) Cyprinid.

Pangolin GRAY 1873 : syn. de Manis LINNE 1758.

Pangolinus RAFINESQUE 1815 : syn. de Manis LINNE 1758.

Panolia GRAY 1843: s./g. de Cervus LINNE 1758.

Panolopus COPE 1861 : R.-SQUAMAT. (Saur.) Anguid.

Panoplites GOULD 1854: Av. APODIF. Trochilid. 
Panotus RAFINESQUE 1815 : P.PERCIF. (Scorpaenoid.) Scorpaenid.

Panterpe CABANIS et HEINE 1860 : Av. APODIF. Trochilid.

Panthera OKEN 1816: M.-FISSIP. Felid.

Pantholops HODGSON 1834: M.PARAX. (Solenodont.) Bovid. (Antilopin.).

Pantodactylus DUMERIL et BIBRON 1839: R.-SQUAMAT. (Saur.) Tejid.

Pantodon PETERS 1876 : P.-CLUPEIF. (Osteoglossoid.) Pantodontid.

Pantolabrus WHITLEY 1931: P. PERCIF. (Percoid.) Carangid.

Pantophos JORDAN et HUBBS 1925: P. -CLUPEIF. (Myctophoid.) Myctophid.

Pantosteus COPE 1875 : P.-CYPRINIF. (Cyprinoid.) Catostomid.

Panturichthys PELLEGRIN 1913 : P. - ANGUILLIF. (Anguilloid.) Hetrenchelyid.

Panugo KOLENATI 1856 : syn. de Nyctalus BOWDICH 1825.

Panurus KOCH 1816: Av. PASSERIF. (Acromyod.) Paradoxornithid.

Panychlora CABANIS et HEINE 1860 : Av. APODIF. Trochilid.

Panyptila CABANIS 1847: Av. APODIF, Trochilid.

Papagomys SODY 1941: syn. de Rattus FISCHER 1803.

Papenua HERRE 1935: P.-PERCIF. (Gobioid.) Gobiid.

Paphosia MULSANT et VERREAUX 1866 : syn. de Lophornis LES. SON.

Papillocheilus SMITH 1945 : P.-CYPRINIF. (Cyprinoid.) Cyprinid.

Papio MULLER 1773 : M.-PRIMAT. (Simioid.) Cercopithecid. (Cercopithecin.).

Pappogeomys MERRIAM 1895 : M.RODENT. (Myomorph.) Geomyid.

Papuservus WHITLEY 1943 : P.PERCIF. (Percoid.) Serranid.

Papyrichthys SMITH 1934 : syn.
d'Icticus JORDAN et THOMPSON 1914.

Papyrocephalus GILBERT et HUBBS 1920 : P.-GADIF. Macrurid.

Parabagrus BLEEKER 1862 : P.CYPRINIF. (Siluroid.) Bagrid.

Parabarbatula MIRANDO-RIBEIRO 1926: Av. PICIF. Capitonid.

Parabarbus FRANZ 1910 : P.-CYPRINIF. (Cyprinoid.) Cyprinid.

Parabelonichthys FOWLER 1943 : P.-SYNGNATHIF. Syngnathid.

Parabembras BLEEKER 1874 : P.PERCIF. (Scorpaenoid.) Platycephalid.

Parablennius MIRANDO-RIBEIRO 1915: syn. de Blennius.

Parabothus NORMAN 1931: P.PLEURONECTIF. (Pleuronectoid.) Bothid.

Parabrachirus MATSUBARA: syn. de Parapterois BLEEKER.

Parabramis BLEEKER 1864: P.CYPRINIF. (Cyprinoid.) Cyprinid.

Parabranchioica DEVINCENZI 1939 : P.-CYPRINIF. (Siluroid.) Trichomyctreid.

Parabrotula ZUGMAYER 1911 : P.PERCIF. (Ophidioid.) Brotulid.

Parabuteo RIDGWAY 1874: syn. d'Erythrocnema SHARPE.

Paracaesio BLEEKER 1874 : P.PERCIF. (Percoid.) Lutjanid.

Paracallionymus BARNARD 1927 : P.-PERCIF. (Callionymoid.) Callionymid.

Paracalotes : R.-SQUAMAT. (Saur.) Agamid.

Paracanthistius BLEEKER 1873 : P.-PERCIF. (Percoid.) Serranid.

Paracanthobrama BLEEKER 1863: P.-CYPRINIF. (Cyprinoid.) Cyprinid.

Paracanthochaetodon SCHMIDT et LINDBERG 1930: P.-PERCIF. (Percoid.) Chaetodontid.

Paracanthopoma GILTAY 1935 : P.-CYPRINIF. (Siluroid.) Trichomycterid.

Paracanthostracion WHITLEY 1933: P.-TETRAODONTIF. (Ostracionoid.) Ostracionid. 
Paracanthurus BLEEKER 1863 : P.-PERCIF. (Acanthuroid.) Acanthurid.

Paracassina PERACCA 1907: Amph. ANOUR. Rhacophorid.

Paracentropogon BLEEKER 1876 : P. - PERCIF. (Scorpaenoid.) Scorpaenid.

Paracentropristes KLUNZINGER 1884: P.-PERCIF. (Percoid.) Serranid.

Paracentroscyllium ALCOCK 1889 : P.-SQUALIF. (Squaloid.) Squalid.

Paraceratias TANAKA 1908 : P.LOPHIIF. (Ceratoid.) Ceratiid.

Paracetopsis (GUICHENOT) BLEEKER 1862 : P.-CYPRINIF. (Siluroid.) Silurid.

Parachaenichthys BOULENGER 1902: P.-PERCIF. (Trachinoid.) Nototheniid.

Parachalcides BOULENGER 1899 : R.-SQUAMAT. (Saur.) Scincid.

Parachela STEINDACHNER 1881: P.-CYPRINIF. (Cyprinoid.) Cyprinid.

Parachaetodon BLEEKER 1874: P.-PERCIF. (Percoid.) Chaetodontid.

Parachaeturichthys BLEEKER 1874: P.-PERCIF. (Gobioid.) Gobiid.

Paracheilognathus BLEEKER 1863: P.-CYPRINIF. (Cyprinoid.) Cyprinid.

Parachromis REGAN 1922 : syn. de Tristamella TREWAVAS 1942.

Paracirrhites STEINDACHNER 1883 : P.-PERCIF. (Percoid.) Cirrhitid.

Paracitharus REGAN 1920: P.PLEURONECTIF. (Pleuronectoid.) Bothid.

Paraclinus MOCQUARD 1889 : P.PERCIF. (Blennioid.) Clinid.

Paracobitis BLEEKER 1863: P.CYPRINIF. (Cyprinoid.) Cobitid.

Paracoelops DORST: M.-CHIROPT. (Microchiropt.) Hipposiderid.

Paraconodon BLEEKER 1876 : P. PERCIF. (Percoid.) Lutjanid.

Paracontias MOCQUARD 1894 : R.-SQUAMAT. (Saur.) Scincid.
Paracophyla MILLOT et GUIBF 1951: Amph. ANOUR. Microhylid.

Parocosia WHITLEY 1958: s./g. d'Amblyapistus.

Paracristiceps HERRE 1939 : P.PERCIF. (Blennioid.) Clinid.

Paracubiceps : P.-PERCIF. (Stromateoid.) Stromateid.

Paracynictis POCOCK 1916 : s./g. de Cynictis OGILBY.

Paradanio BLEEKER 1863 : P.-CYPRINIF. (Cyprinoid.) Cyprinid.

Paradelma KINGHORN 1926 : R.SQUAMAT. (Saur.) Pygopodid.

Parademansia KINGHORN 1955: R.SQUAMAT. (Serpent.) Elapid.

Paradicichthys WHITLEY 1930 : P.-PERClF. (Percoid.) Lutjanid.

Paradicrolene ALCOCK 1889 : P.PERCIF. (Ophidioid.) Brotulid.

Paradicula WHITLEY 1931: P.PLEURONECTIF. (S o l e o $i d$.) Soleid.

Paradigalla LESS ON 1835:Av. PASSERIF. (Acromyod.) Paradiseid. (Paradisein.).

Paradiplomystes BLEEKER 1862: P.-CYPRINIF, (Siluroid.) Bagrid.

Paradiplomystax GUNTHER 1864: emend. pro Paradiplomystes $B$. 1862.

Paradipus VINOGRADOV 1930 : M.-RODENT. (Myomorph.) Dipodid. (Dipodin.).

Paradiretmus WHITLEY 1948 : P.BER YCIF. Diretmid.

Paradisea LINNE 1758: Av. PASSERIF. (Acromyod.) Paradiseid. (Paradisein.).

Paradisornis FINSCH et MEYER 1885: Av. PASSERIF. (Acromyod.) Paradiseid. (Paradisein.).

Paradistichodus PELLEGRIN 1922: P.-CYPRINIF. (Characoid.) Characid.

Paradolichotis KRAGLIEVITCH 1927: s./g. de Dolichotis DESMAREST 1819.

Paradon DESMAREST 1874 : vide Parodon C. et V. 1849. 
Paradoxichthys GIGLIOLI 1882 : P. - NOTACANTHIF. Notacanthid.

Paradoxodon WAGLER 1855 : syn. de Suncus EHRENBERG 1833.

Paradoxornis GOULD 1836: Av. PASSERIF. (Acromyod.) Paradoxornithid.

Paradoxurus CUVIER 1821: M.FISSIP. Viverrid. (Paradoxurin.).

Paradules BLEEKER 1863: P.PERCIF. (Percoid.) Serranid.

Paraechinus TROUESSART 1879 : M.-INSECTIV. Erinaceid. (Erinacein.).

Paragalaxias SCOTT 1934: P.CLUPEIF. (Galaxioid.) Galaxiid.

Paragaleus BUDKER 1935 : P.-GALEIF. (Carcharhinoid.) Carcharhinid.

Paragambusia MEEK 1904 : P.-CYPRINODONTIF. Poeciliid.

Paragehyra ANGEL 1929: R.SQUAMAT. (Saur.) Geckonid.

Parageophis BOCOURT 1883 : R.SQUAMAT. (Serpent.) Colubrid.

Paraglossa HODGSON : Av. PASSERIF. (Acromyod.) Sturnid. (Graculin.).

Paraglyphidodon BLEEKER 1876 : P.-PERCIF. (Labroid.) Pomacentrid.

Paragobiodon BLEEKER 1874 : P.PERCIF. (Gobioid.) Gobiid.

Paragobioides KENDALL et GOLDSBOROUGH 1911 : P.PERCIF. (Gobioid.) Gobiid. (ou Microdesmid.).

Paragobiopsis KOUMANS 1941: P.-PERCIF. (Gobioid.) Gobiid.

Paragobius BLEEKER 1873: P.PERCIF. (Gobioid.) Gobiid.

Paragonatodes NOBLE 1921: syn. de Cnemaspis STRAUCH 1887.

Paragoniates STEINDACHNER 1877: P.-CYPRINIF. (Characoid.) Characid.

Paragonus GILL 1862 : P.-PERCIF. (Cottoid.) Agonid.

Parahelicops BOURRET 1934 : R.SQUAMAT. (Serpent.) Colubrid.
Parahemiodon BLEEKER 1863: P.-CYPRINIF. (Siluroid.) Loricariid.

Paraheminodus : P. PERCIF. (Blennioid.) Blenniid.

Parahemiodus : P.-CYPRINIF. (Characoid.) Characid.

Parahollardia FRASER-BRUNNER 1941 : P.-PERCIF. (Balistoid.) Triacanthid.

Parahydromys POCHE 1906: M.RODENT. (Myomorph.) Murid. (Hydromyin.).

Parahynnodus BARNARD 1927: P.-PERCIF, (Percoid.) Serranid.

Parailia BOULENGER 1899 : P.CYPRINIF. (Siluroid.) Schilbeid.

Parakuhlia PELLEGRIN 1913 : P.PERCIF. (Percoid.) Serranid.

Parakysis HERRE 1940 : P.-CYPRINlF. (Siluroid.) Bagrid.

Paralabidochromis GREENWOOD 1956 : P.-PERCIF. (Percoid.) Cichlid.

Paralabrax GIRARD 1856 : P.PERCIF. (Percoid.) Serranid.

Paralates SAUVAGE 1883 : syn. de Lates CUVIER et VALENCIENNES.

Paralaubuca BLEEKER 1865 : P.CYPRINIF. (Cyprinoid.) Cyprinid.

Paralces ALLEN 1902: syn. de Alces GRAY 1821.

Paralepidocephalus TCHANG 1935: P.-CYPRINIF. (Cyprinoid.) Cobitid.

Paralepis RISSO 1820: P.-CLUPEIF. (Myctophoid.) Sudid. ou Paralepidid.

Paraleptomys : M.-RODENT. (Myomorph.) Murid. (Hydromyin.).

Paraleucogobio BERG 1907: P.CYPRINIF. (Cyprinoid.) Cyprinid.

Paralichthodes GILCHRIST 1902: P.-PERCIF. (Percoid.) Maenid.

Paralichthys GIRARD 1858: P.PLEURONECTIF. (Pleuronectoid.) Bothid.

Paralimanda BREDER 1927: syn. de Poecilopsetta GUNTHER 1880. 
Paralinurichthys : P.-PLEURONECTIF. (Pleuronectoid.) Bothid.

Paraliparis COLLETT 1878: P.PERCIF. (Cottoid.) Cyclopterid.

Paralonchurus BOCOURT 1869 : P.-PERCIF. (Percoid.) Sciaenid.

Paraluteres BLEEKER 1866 : P.PERCIF. (Balistoid.) Balistid.

Paralygosoma CHABANAUD 1917 : s.jg. de Lygosoma GRAY.

de Varanus RAFINESQUE

Paramacrurus BLEEKER 1874: P.-GADIF. Macrurid.

Paramanis POCOCK $1924: s . / g$. de Manis LINNE 1758.

Parambassis BLEEKER 1874 : P.PERCIF. (Percoid.) Serranid.

Parameles : errat. pro Perameles GEOFFROY-ST-HILAIRE.

Paramelomys RUMMLER 1936 : s. $/ g$. de Melomys THOMAS 1922.

Parameriones HEPTNER 1937: $s . / g$. de Meriones ILLIGER 1811.

Paramesotriton CHANG 1935 : Amph. UROD. Salamandrid.

Paramia BLEEKER 1863 : P.-PERCIF. (Percoid.) Serranid.

Paramicrogale GRANDIDIER et PETIT: syn. de Microgale THOMAS 1882.

Paraminous FOWLER 1943 : P.PERCIF. (Scorpaenoid.) Scorpaenid.

Paramisgurnus SAUVAGE 1878: P.-CYPRINIF. (Cyprinoid.) Cobitid.

Paramonacanthus STEINDACHNER 1867: syn. de Laputa WHITLEY 1930.

Paramonacanthus BLEEKER 1866: P.-PERCIF. (Balistoid.) Balistid. (ou Monacanthid.).

Paramphilius PELLEGRIN 1907: P.-CYPRINIF. (Siluroid.) Silurid.

Paramphiprion : P.-PERCIF. (Labroid.) Pomacentrid.

Paramyloplus NORMAN 1929 : P.CYPRINIF. (Characoid.) Characid.
Paramyomyrus PELLEGRIN 1927 : P.-CLUPEIF. (Mormyroid.) Mormyrid.

Paramyotis BIANCHI 1916: s./g. de Myotis KAUP 1829.

Paramyrus GUNTHER 1870 : P.ANGUILLIF. (Congroid.) Echelid.

Paramyxine DEAN 1904: Cyclost. $M Y X I N I F$. Myxinid.

Paranaja LOVERIDGE 1944: R.SQUAMAT. (Serpent.) Elapid.

Paranandus DAY 1865: P.-PERCIF. (Percoid.) Nandid.

Paranectris IREDALE 1930 : s./g. de Neonectris.

Paraneltroplus REGAN 1905: P.PERCIF. (Percoid.) Cichlid.

Paranotropis FOWLER 1904 : P.CYPRINIF. (Cyprinoid.) Cyprinid.

Parantechinus TATE 1947: M.MARSUP. Dasyurid. (Dasyurin.).

Paranthias GUICHENOT 1868 : P.PERCIF. (Percoid.) Serranid.

Paranyctemene TATE : M.-CHIROPT. (Megachiropt.) Pteropid. (Nyctemenin.).

Paraonyx HINTON 1921 : M.-FISSIP. Mustelid. (Lutrin.).

Paraoxyrhopus : errat. pro Paroxyrhopus SCHENKEL 1901.

Parapegasus (BLEEKER) DUMERIL 1870 : P.-PERCIF. (Pegasoid.) Pegasid.

Parapelecus GUNTHER 1889 : P.CYPRINIF. (Cyprinoid.) Cyprinid.

Parapercis STEINDACHNER 1884: P.-PERCIF. (Trachinoid.) Pinguipedid.

Parapetenia BOULENGER 1905: P.-PERCIF. (Percoid.) Cichlid.

Paraphago BOULENGER 1899 : P.CYPRINIF. (Characoid.) Characid.

Paraphotichthys WHITLEY 1931: P.-CLUPEIF. (Stomiatoid.) Gonostomid.

Paraphoxinus BLEEKER 1863: syn. de Phoxinellus HECKEL 1843.

Paraphractura BOULENGER 1902: P.-CYPRINIF. (Siluroid.) Schilbeid. (ou Bagrid.). 
Paraphrynonax LUTZ et MELLO 1920: syn. de Synchalinus COPE 1894.

Paraphya MUNRO 1949: P.-PERCIF. (Gobioid.) Gobiid.

Parapimelodus LAMONTE 1933 : P.-CYPRINIF. (Siluroid.) Pimelodid.

Parapistocalamus ROUX 1934 : R.SQUAMAT. (Serpent.) Elapid.

Paraplagusia BLEEKER 1866 : P.PLEURONECTIF. (Soleoid.) Cynoglossid.

Paraplatyglossus BLEEKER 1878 : P.-PERCIF. (Labroid.) Labrid.

Paraplectes WOLTERS 1943: $s . / g$. d'Euplectes SWAINSON.

Paraplesiops BLEEKER 1875 : P.PERCIF. (Percoid.) Plesiopid.

Paraplotosus : P.-CYPRINIF. (Siluroid.) Plotosid.

Parapocyptes BLEEKER 1874 : P.PERCIF. (Gobioid.) Gobiid.

Parapoecilia HUBBS 1925 : P.-CYPRINODONTIF. Poeciliid.

Parapomacentrus BLEEKER 1877: P.-PERCIF. (Labroid.) Pomacentrid.

Parapostolepis AFRANIO do AMARAL 1930 : R.-SQUAMAT. (Serpent.) Boigid.

Parapriacanthus STEINDACHNER 1870: P.-PERCIF. (Percoid.) Pempherid.

Parapristipoma BLEEKER 1873 : P.-PERCIF. (Percoid.) Lutjanid.

Parapristiurus FOWLER 1934: s./g. de Pristiurus BONAPARTE 1831.

Paraprocypris : P.-CYPRINIF. (Cyprinoid.) Cyprinid.

Paraprotomyzon PELLEGRIN et F A N G 1935 : P.-CYPRINIF. (Cyprinoid.) Homalopterid.

Paraprotosalanx FANG 1934: P.CLUPEIF. (Salmonoid.) Salangid.

Parapsettus STEINDACHNER 1876 : P.-PERCIF. (Percoid.) Chaetodontid.

Parapseudecheneis HORA 1930 : P.-CYPRINIF, (Siluroid.) Bagrid.
Parapterois BLEEKER 1876: P.PERCIF. (Scorpaenoid.) Scorpaenid.

Parapterygotrigla : P.-P ERCIF. (Scorpaenoid.) Triglid.

Paraptychodus CARTER: syn. de Alloptychodus WHITLEY 1953.

Pararasbora REGAN 1908: P.-CYPRINIF. (Cyprinoid.) Cyprinid.

Parargyrops TANAKA 1916: syn. d'Argyrops.

Pararhabdophis BOURRET 1934 : R.-SQUAMAT. (Serpent.) Colubrid.

Pararhadinea BOETTEGER 1898 : R.-SQUAMAT. (Serpent.) Colubrid.

Pararhodeus BERG 1907: s./g. de Phoxinellus HECKEL 1843.

Pararhynchobdella BLEEKER 1874 : P. - MASTACEMBELIF. Mastacembelid.

Pararius WHITLEY 1940 : P.-CYPRINIF. (Siluroid.) Bagrid.

Parartedius HUBBS 1926 : P.-PERCIF. (Cottoid.) Cottid.

Pararthroleptis AHL 1923 : Amph. ANOUR. Ranid.

Pararutilus BERG 1912 : P.-CYPRINIF. (Cyprinoid.) Cyprinid.

Parasalanx REGAN 1908: P.-CLUPEIF. (Salmonoid.) Salangid.

Parascalops TRUE 1894: M.-INSECTIV. Talpid. (Scalopin.).

Parascapirhynchus FORBES et RICHARDSON 1905: P.-ACIPENSERIF. Acipenserid.

Parascaptor GILL 1875: syn. de Talpa LINNE 1766.

Paraschizothorax BLEEKER 1863 : P.-CYPRINIF. (Cyprinoid.) Cyprinid.

Parasciurus TROUESSART 1880 : s./g. de Sciurus LINNE 1758.

Parascolopsis BOULENGER 1901: P.-PERCIF. (Percoid.) Lutjanid.

Parascombrops ALCOCK 1890 : P.-PERCIF. (Percoid.) Serranid.

Parascorpaena BLEEKER 1876 : P.-PERCIF. (Scorpaenoid.) Scorpaenid. 
Parascorpis BLEEKER 1875: P.PERCIF. (Percoid.) Chaetodontid.

Parascotomanes BOURRET 1942 : syn. de Scotophilus LEACH 1821.

Parascyllium GILL 1861: P.-GALEIF. (Isuroid.) Orectolobid.

Paraserranus BLEEKER 1874: P.PERCIF. (Percoid.) Serranid.

Paraserrivomer ROULE et ANGEL 1931: syn. de Serrivomer GILL et RYDER 1883.

Paraserrivomer ROULE et BERTIN 1933 : P.-ANGUILLIF. (Nemichthyoid.) Serrivomerid.

Parasilurus BLEEKER 1862: P.CYPRINIF. (Siluroid.) Silurid.

Parasinilabeo : P.-CYPRINIF. (Cyprinoid.) Cyprinid.

Parasphaerichthys PRASHAD et MUKERJI 1929: P.-PERCIF. (Anabantoid.) Anabantid.

Parasphenanthias GILCHRIST 1923: P.-PERCIF. (Percoid.) Serranid. (ou Opisthognathid.).

Parasphyraenops BEAN 1912 : P.PERCIF. (Percoid.) Serranid.

Parastathmonotus CHABANAUD 1942: P.-PERCIF. (Blennioid.) Clinid.

Parastegophilus de MIRANDO-ROBEIRO 1946 : P. - CYPRINIF. (Siluroid.) Trichomycterid.

Parastomias ROULE et BERTIN 1931: P. -CLUPEIF. (Stomiatoid.) Melanostomiatid.

Parastrolytes HUBBS 1926 : P.PERCIF. (Cottoid.) Cottid.

Parastromateus BLEEKER 1865 : P.-PERCIF. (Stromateoid.) Stromateid.

Parasyngnathus : s./g. de Syngnathus LINNE 1758.

Parataeniophorus BERTELSEN et MARSHALL 1956: P.-CLUPEIF. (Miripinnoid.) Eutaeniophorid.

Paratapinophis ANGEL 1929 : R.SQUAMAT. (Serpent.) Colubrid.

Parateleopus RADCLIFFE 1912 : P.-CLUPEIF. (Ateleopoid.) Ateleopid.

Paratelmatobius LUTZ et LEITAO de CARVALHO 1958: Amph. ANOUR. Leptodactylid.
Paratetradactylus ANGEL 1922 : R.-SQUAMAT. (Saur.) Gerrhosaurid.

Paratherina A URICH 1935 : P.MUGILIF. Atherinid.

Parathunnus KISHINOUYE 1923 : P.-PERCIF. (Scombroid.) Thunnid.

Paratilapia B L E E K E R 1868 : P.PERCIF. (Percoid.) Cichlid.

Paratrachichthys WAITE 1899 : P.BERYCIF. Trachichthyid.

Paratractus GILL 1863: P.-PERCIF. (Percoid.) Carangid.

Paratriacanthodes FOWLER 1934 : P.-PERCIF. (Balistoid.) Triacanthid.

Paratrigla OGILBY 1911 : P.-PERCIF. (Scorpaenoid.) Triglid.

Paratylognathus SAUVAGE 1880 : P.-CYPRINIF. (Cyprinoid.) Cyprinid.

Paratyntastes GILTAY 1935: P.PERCIF. (Gobioid.) Gobiid.

Parauchenipterus BLEEKER 1862 : P.-CYPRINIF. (Siluroid.) Doradid.

Parauchenoglanis BOULENGER 1911 : P.-CYPRINIF. (Siluroid.) Bagrid.

Paravacara : errat. pro Parvacara.

Paravandellia RIBEIRO 1912 : P.CYPRINIF. (Siluroid.) Trichomycterid.

Paraxenomystax REID 1940 : P.ANGUILLIF. (Congroid.) Congrid. (ou Muraenesocid.).

Paraxerus FORSYTH MAJOR 1893: M. -RODENT. (Sciuromorph.) Sciurid. (Sciurin.).

Parazenopsis CLIGNY 1909: P.ZEIF. Zeid.

Pardachirus GUNTHER 1862 : syn. d'Achirus de LACEPEDE 1802.

Pardaliparus SELYS-LONGCHAMPS 1884 : Av. PASSERIF. (Acromyod.) Parid.

Pardalotus VIEILLOT 1816: Av. PASSERIF. (Acromyod.) Dicaeid.

Pardictis THOMAS 1925 : s./g. de Prionodon HORSFIELD 1822.

Pardina KAUP 1829 : syn. de Felis LINNE 1758. 
Pardirallus BONAPARTE 1856 : Av. RALLIF. Rallid.

Pardofelis SEVERTZOV $1858: \mathrm{s} . / \mathrm{g}$. de Felis LINNE 1758.

Parduliparus : errat. pro Pardaliparus SELYS-LONGCHAMPS.

Pardus FITZINGER 1868 : syn. de Panthera OKEN 1816.

Pareas WAGLER 1830 : syn. d'Amblycephalus KUHL.

Parecbasis EIGENMANN 1914 : P.CYPRINIF. (Characoid.) Characid.

Pareiodon KNER 1855 : P.-CYPRINIF. (Siluroid.) Trichomycterid.

Pareiorhaphis RIBEIRO 1918 : P.CYPRINIF. (Siluroid.) Loricariid.

Pareiorhina GOSLINE 1848 : P.-CYPRINIF. (Siluroid.) Loricariid.

Paremblemaria LONGLEY 1928: P.-PERCIF. (Blennioid.) Blenniid.

Parenephelus : errat. pro Parepinephelus.

Parephippus GILL 1862: P.-PERCIF. (Percoid.) Ephippid.

Parepinenhelus BLEEKER 1876: P.-PERCIF. (Percoid.) Serranid.

Pareptesicus BIANCHI 1916: syn. d'Eptesicus RAFINESQUE 1820.

Pareques (GILL) GOODE 1876 : P.PERCIF. (Percoid.) Sciaenid.

Parequula STEINDACHNER 1879 : P.-PERCIF. (Percoid.) Leiognathid.

Paretroplus BLEEKER 1868: P.PERCIF. (Percoid.) Cichlid.

Pareudiastes HARTLAUB et FINSCH 1871: Av, RALLIF, Rallid.

Pareustomias BAILLY 1930 : P.CLUPEIF. (Stomiatoid.) Stomiatid.

Pareutropius REGAN 1920 : P.-CYPRINIF. (Siluroid.) Schilbeid.

Parexocoetoides : $P .-B E L O N I F$. (Exocoetoid.) Exocoetid.

Parexocoetus BLEEKER 1866 : P.BELONIF. (Exocoetoid.) Exocoetid.
Parexoglossum HUBBS 1931: P.CYPRINIF. (Cyprinoid.) Cyprinid.

Parexostoma REGAN 1905 : P.-CYPRINIF. (Siluroid.) Bagrid.

Parhaplodactylus THOMINOT 1883: P.-PERCIF. (Percoid.) Aplodactylid.

Parhomaloptera VAILLANT 1902 : P.-CYPRINIF. (Cyprinoid.) Homalopterid.

Parhoplophryne BARBOUR et LOVERIDGE 1928： Amph. ANOUR. Microhylid.

Paricelinus EIGENMANN 1889 : P.PERCIF. (Cottoid.) Cottid.

Parika WHITLEY 1955: P.-PERCIF. (Balistoid.) Balistid.

Pariodon GUNTHER 1864 : P.-CYPRINIF. (Siluroid.) Trichomycterid.

Parioglossus REGAN 1912 : P.-PERCIF. (Gobioid.) Eleotrid.

Pariolius COPE 1872: P.-CYPRINIF. (Siluroid.) Trichomycterid.

Parisoma SWAINSON 1832: Av. PASSERIF. (Acromyod.) Muscicapid.

Paristiopterus BLEEKER 1876 : P.PERCIF. (Percoid.) Lutjanid. (ou Histiopterid.).

Parkerophis BARBOUR et AFRANIO do AMARAL 1927: syn. de Chilorhinophis WERNER 1908 .

Parkia FOWLER 1904 : P.-PERCIF. (Percoid.) Lutjanid.

Parma GUNTHER 1862: P.-PERCIF. (Labroid.) Pomacentrid.

Parmaturus GARMAN 1906 : P.GALEIF. (Carcharhinoid.) Scyliorhinid.

Parmoptila CASSIN 1859 : Av. PASSERIF. (Acromyod.) Dicaeid.

Paroaria BONAPARTE 1831: Av. PASSERIF. (Acromyod.) Fringillid. (Pitylin.).

Parochusus WHITLEY 1930 : P.PERCIF. (Percoid.) Leiognathid.

Parodon CUVIER et VALENCIENNES 1849 : P.-CYPRINIF. (Characoid.) Characid.

Parodontops SCHULTZ et MILES 
1943 : P.-CYPRINIF. (Characoid.) Characid.

Paroligosarcus AMARAL et TREWAVAS 1949 : s./g. d'Oligosarcus GUNTHER.

Paroneirodes ALCOCK 1890: P.LOPHIIF. (Ceratoid.) Oneirodid.

Parophidion TORTONESE 1954: P.-PERCIF. (Ophidioid.) Ophidiid.

Parophiocephalus POPTA 1905: P.-PERCIF. (Anabantoid.) Anabantıd.

Parophrys GIRARD 1856: P.-PLEURONECTIF. (Pleuronectoid.) Pleuronectid.

Paropsis JENYNS 1841: P.-PERCIF. (Percoid.) Carangid.

Parosphromenus BLEEKER 1879 : P.-PERCIF. (Anabantoid.) Anabantid.

Parosteobrama TCHANG 1930 : P.CYPRINIF. (Cyprinoid.) Cyprinid.

Parotia VIEILLOT 1816 : Av. PASSERIF. (Acromyod.) Paradiseid. (Paradisein.).

Parotocinclus EINGENMANN 1889: P.-CYPRINIF. (Siluroid.) Loricariid.

Parotomys THOMAS 1918 : M.-RODENT. (Myomorph.) Murid. (Otomyin.).

Paroxyrhopus SCHENKEL 1901: R.-SQUAMAT. (Serpent.) Boigid.

Paroxyurichthys BLEEKER 1875 : P.-PERCIF. (Gobioid.) Gobiid.

Parra LINNE 1766 : syn. de Jacana BRISSON.

Parrella GINSBURG 1938 : P.-PERCIF. (Gobioid.) Gobiid.

Parrichthys BARBOUR 1941: P. LOPHIIF. (Ceratoid.) Ceratiid.

Parula BONAPARTE 1838: Av. PASSERIF. (Acromyod.) Parulid.

Parulus RAFINESQUE 1815: Av. PASSERIF. (Acromyod.) Parulid.

Parupeneus KLUNZINGER 1887 : P.-PERCIF. (Percoid.) Mullid.

Paruromys ELLERMAN $1954: s . / g$. de Rattus FRISCH 1803.
Parus LINNE 1758: Av. PASSERIF. (Acromyod.) Parid.

Parvacara : P.-PERCIF. (Percoid.) Cichlid.

Parviclinus FRASER-BRUNNER 1933 : P.-PERCIF. (Blennioid.) Clinid.

Parvicrepis WHITLEY 1931: P.PERCIF. (Gobiesoxoid.) Gobiesocid.

Parvigobius WHITLEY 1930: P.PERCIF. (Gobioid.) Gobiid.

Parvimolge TAYLOR: Amph. UROD. Plethodontid.

Parviparma HERRE 1927 : P.-PERCIF. (Gobioid.) Eleotrid.

Parvoraja: P.-RAJIF. (Rajoid.) Rajid.

Parvulus LUTZ 1930 : s./g. de Leptodactylus FITZINGER.

Paryphephorus MEYER 1891: Av. PASSERIF. (Acromyod.) Paradiseid. (Paradisein.).

Paschalestes G IS T E L 1848 : P.PERCIF. (Percoid.) Serranid.

Passer BRISSON 1760 : Av. PASSERIF. (Acromyod.) Ploceid (Passerin.).

Passer WALBAUM 1792: syn. de Platessa.

Passerculus SPIX 1824: Av. PASSERIF. (Acromyod.) Ploceid. (Passerin.).

Passerella SWAINSON 1837: Av. PASSERIF. (Acromyod.) Fringillid. (Emberizin.).

Passerherbulus MAYNARD 1895 : Av. PASSERIF. (Acromyod.) Fringillid.

Passerina VIEILLOT 1816: Av. PASSERIF. (Acromyod.) Fringillid. (Pitylin.).

Passerita GRAY 1825 : syn. d'Ahaetulla LINK.

Pastinaca SWAINSON 1838: syn. de Dasyatis.

Pastinachus RUPPELL 1828: syn. de Dasyatis.

Pastor TEMMINCK 1815 : Av. PASSERIF. (Acromyod.) Sturnid. (Sturnin.).

Pataecus RICHARDSON 1844: P.PERCIF. (Scorpaenoid.) Pataecid. 
Patagona GRAY 1840: Av. APODIF. Trochilid.

Paulicea IHERING 1898: P.-CYPRINIF. (Siluroid.) Bagrid.

Pauloscirtes WHITLEY 1935 : P.PERCIF. (Blennioid.) Blenniid.

Paurodus SCHULZE 1897 : syn. de Crocidura WAGLER 1832.

Pauxis SCLATER 1875: Av. PHASIANIF. Cracid.

Pavo LINNE 1758: Av. PHASIANIF. Phasianid. (Phasianin.).

Pavoclinus : P.-PERCIF. (Blennioid.) Clinid.

Pavoncella LEACH 1816 : syn. de Philomachus.

Pawnurus PARR 1946 : s./g. de Malacocephalus GUNTHER.

Pealea RIDGWAY 1886: Av. PROCELLARIIF. Hydrobatid.

Pealeornis MATHEWS 1932: Av. PROCELLARIIF. Hydrobatid.

Peaolopesia SMITH 1949 : P.-PERCIF. (Labroid.) Labrid.

Pecari REICHENBACH 1835 : syn. de Dicotyles CUVIER.

Pectinator BLYTH 1855: M.-RODENT. (Sciuromorph.) Ctenodactylid.

Pedalibrycon FOWLER 1943 : P.CYPRINIF. (Characoid.) Characid.

Pedetes ILLIGER 1811 : M.-RODENT. (Theridomyomorph.) Pedetid.

Pedilorhynchus REICHENOW 1891: Av. PASSERIF. (Acromyod.) Muscicapid.

Pediocaetes BAIRD 1858: Av. PHASIANIF. Tetraonid.

Pediolagus MARELLI 1927: syn. de Dolichotis DESMAREST 1819 .

Pedionomus GOULD 1840: Av. RALLIF. Turnicid.

Pediopipo SUNDEVALL 1866: Av. PASSERIF. (Mesomyod.) Piprid.

Pediporus ROUX 1931: R.-SQUAMAT. (Saur.) Scincid.

Pedites GISTEL 1848 : P.-PERCIF. (Anabantoid.) Anabantid.

Pedomys BAIRD 1857: M.-RODENT. (Myomorph.) Microtid.
Pedostibes GUNTHER 1876 : Amph. ANOUR. Bufonid.

Pegasus LINNE 1758 : P.-PERCIF. (Pegasoid.) Pegasid.

Pegedictis RAFINESQUE 1820 : P.PERCIF. (Cottoid.) Cottid.

Pegusa GUNTHER 1862 : P.-PLEURONECTIF. (Soleoid.) Soleid.

Pelagios CUVIER 1824: syn. de Monachus FLEMMING 1822.

Pelaxocyon GLOGER 1841 : syn. de Monachus FLEMMING 1822.

Pelagodroma REICHENBACH 1852: Av. PROCELLARIIF. $\mathrm{Hy}$ drobatid.

Pelamis DAUDIN 1803: syn. de Hydrus SCHNEIDER.

Pelamydrus STEJNEGER 1910 : R.-SQUAMAT. (Serpent.) Hydrophiid.

Pelamys CUVIER et VALENCIENNES : P.-PERCIF. (Scombroid.) Scombrid.

Pelargopsis GLOGER 1841: Av. CORACIADIF. Alcedinid.

Pelates CUVIER et VALENCIENNES 1829: P.-PERCIF. (Percoid.) Serranid.

Pelea GRAY 1850 : M.-PARAX. (Solenodont.) Bovid. (Reduncin.).

Pelecanichthys GILBERT et CRAMER 1896 : P.-PLEURONECTIF. (Pleuronectoid.) Bothid.

Pelecanoides de LACEPEDE 1799 : Av. PROCELLARIIF. Pelecanoidid.

Pelecanus LINNE 1758: Av. PELECANIF. Pelecanid.

Pelecinomimus : syn. de Cetomimus GOODE et BEAN 1894.

Pelecus AGASSIZ 1836 : P.-CYPRINIF. (Cyprinoid.) Cyprinid.

Peleunomimus : errat. pro Pelecinomimus.

Pelgadis : errat. pro Plegadis KAUP.

Pelias MERREM 1820 : syn. de Vipera GRONOV.

Pelidna CUVIER 1817 : syn. de Calidris ILLIGER 1811.

Peliocichla CABANIS 1882: Av. PASSERIF. (Acromyod.) Turdid. 
Pellegrinina FOWLER 1906 : P.-CYPRINIF. (Characoid.) Characid.

Pellisolus PARR 1951: P.-CLUPEIF. (Alepocephaloid.) Alepocephalid.

Pellochromis FOWLER et BEAN 1928: P.-PERCIF. (Labroid.) Pomacentrid.

Pellona CUVIER et VALENCIENNES 1847: syn. de Ilisha (GRAY) RICHARDSON 1845.

Pellonula GUNTHER 1868 : P.-CLUPEIF. (Clupeoid.) Clupeid.

Pellonulops SMITH 1949 : P.-CLUPEIF. (Clupeoid.) Clupeid.

Pellorneum SWAINSON 1832: Av. PASSERIF. (Acromyod.) Timaliid.

Pelmatochromis STEINDACHNER 1894: P.-PERCIF. (Percoid.) Cichlid.

Pelobates WAGLER 1830: Amph. ANOUR. Pelobatid.

Pelochelys GRAY 1864 : R.-TESTUDIN. (Thecoph.) Trionychid.

Pelodytes BONAPARTE 1841: Amph. ANOUR. Pelobatid.

Pelomedusa WAGLER 1830 : R.TESTUDIN. (Thecoph.) Pelomedusid.

Pelomys PETERS 1852: M.-RODENT. (Myomorph.) Murid. (Murin.).

Pelophilus DUMERIL et BIBRON $1844:$ R.-SQUAMAT. (Serpent.) Boid.

Pelophryne: Amph. ANOUR. Bufonid.

Pelopsia FACCIOLA 1883 : P.-CLUPEIF. (Myctophoid.) Synodid.

Pelor CUVIER et VALENCIENNES 1829 : P. -PERCIF. (Scorpaenoid.) Synancejid.

Peloria COCCO 1844 : P.-PLEURONECTIF. (Pleuronectoid.) Bothia.

Peloropsis GILBERT 1905 : P.-PERCIF. (Scorpaenoid.) Synancejid.

Pelothrophus GUNTHER 1864: voir Peletrophus.

Pelotretis WAITE 1911 : P.-PLEURONECTIF. (Pleuronectoid.) Pleuronectid.
Pelsartia : P.-PERCIF. (Percoid.) Serranid.

Peltapleura FOWLER 1906 : P.CYPRINIF. (Characoid.) Characid.

Peltatetraops FOWLER 1931 : s./g. d'Anableps BLOCH.

Pelteobagrus BLEEKER 1864 : P.CYPRINIF. (Siluroid.) Bagrid.

Peltharpodon FOWLER 1934 : P.CLUPEIF. (Myctophoid.) Synodid.

Peltohyas SHARPE 1896 : Av. CHARADRIIF. Charadriid. (Peltohyatin.).

Peltops WAGLER 1829: Av. PASSERIF. (Acromyod.) Muscicapid.

Peltorhamphus GUNTHER 1862 : P. - PLEURONECTIF. (Pleuronectoid.) Pleuronectid.

Peltorhinus PETERS 1876 : syn. de Stenoderma GEOFFROY-STHILAIRE.

Peltorneum : vide Pellorneum SWAINSON.

Peltrypops : errat. pro Plectrypops.

Pelusios WAGLER 1830 : R.-TESTUDIN. (Thecoph.) Pelomedusid.

Pempherichthys KLUNZINGER 1871: P.-PERCIF. (Percoid.) Pempherid.

Pempheris CUVIER 1829 : P.-PERCIF. (Percoid.) Pempherid.

Peneapocryptes : P.-PERCIF. (Gobioid.) Gobiid.

Penelope LINNE 1758: Av. PHASIANIF. Cracid.

Penelopides REICHENBACH 1849 : Av. CORACIADIF. Bucerotid.

Penelopina REICHENBACH 1862 : Av. PHASIANIF, Cracid.

Peneoenanthe MATHEWS 1920 : Av. PASSERIF. (Acromyod.) Muscicapid.

Peneothello: Av. PASSERIF. (Acromyod.) Muscicapid.

Penesilurus HERRE 1924 : P.-CYPRINIF. (Siluroid.) Silurid.

Penetopteryx LUNEL 1881 : P.SYNGNATHIF. Syngnathid.

Penetroptery : errat pro Penetopteryx LUNEL 1881. 
Penguinus BRUNNICH 1772 : syn. de Pinguinus.

Penicipelta : P.-PERCIF. (Balistoid.) Balistid.

Pennon WHITLEY 1941 : P.-PERCIF. (Percoid.) Mullid.

Pennula DOLE 1879: Av. RALLIF. Rallid.

Penopus GOODE et BEAN 1896 : P.-PERCIF. (Ophidioid.) Brotulid.

Pentaceropsis STEINDACHNER et DODERLEIN 1903: P.-PERCIF. (Percoid.) Lutjanid.

Pentaceros CUVIER et VALENCIENNES 1829 : P.-PERCIF. (Percoid.) Lutjanid.

Pentalagus LYON 1903: M.-LAGOMORPH. Leporid. (Palaeolagin.).

Pentanchus SMITH et RADCLIFFE 1912: P.-GALEIF. (Carcharhinoid.) Scyliorhinid.

Pentanemus GUNTHER 1860: P.POLYNEMIF. Polynemid.

Pentapodus QUOY et GAIMARD 1824: P.-PERCIF. (Percoid.) Lutjanid.

Pentaprion BLEEKER 1850 : P.-PERCIF. (Percoid.) Leiognathid.

Pentapus CUVIER et VALENCIENNES 1830: P.-PERCIF. (Percoid.) Lutjanid.

Pentaroge GUNTHER 1860: P.PERCIF. (Percoid.) Chaetodontid.

Pentherichthys REGAN et TREWAVAS 1932: P. - LOPHIIF. (Ceratoid.) Himantolophid.

Penthesthes REICHENBACH 1850 : syn. de Parus LINNE 1758.

Penthetor ANDERSEN 1912: M.CHIROPT. (Megachiropt.) Pteropid. (Pteropin.).

Penthetria CABANIS 1847: Av. PASSERIF. (Acromyod.) Ploceid. (Viduin.).

Penthetriopsis SHARPE 1890: Av. PASSERIF. (Acromyod.) Ploceid. (Viduin.).

Penthoceryx CABANIS et HEINE 1863: Av. CUCULLIF. Cucullid.

Pentholaea CABANIS 1850: Av.
PASSERIF. (Acromyod.) Turdid.

Pephraeops : errat. pro Tephraeops GUNTHER 1859.

Pepoasa LESSON 1837: Av. PASSERIF. (Mesomyod.) Tyrannid.

Peprilus CUVIER 1829: P.-PERCIF. (Stromat.) Stromateid.

Peradorcas THOMAS 1904: M.MARSUP. Macropodid. (Macropodin.).

Peragalia GRAY 1841 : syn. de Perameles GEOFFROY-ST-HILAIRE 1803.

Perameles GEOFFROY-ST-HILAIRE 1803 : M.-MARSUP. Peramelid.

Peramys LESSON 1842: syn. de Monodelphis BURNETT 1830.

Perca LINNE 1758 : P.-PERCIF. (Percoid.) Percid.

Percalabrax TEMMINCK 1850 : P.PERCIF. (Percoid.) Serranid.

Percalates RAMSAY et OGILBY 1887: P.-PERCIF. (Percoid.) Serranid.

Percamia BLEEKER 1876 : P.-PERCIF. (Percoid.) Serranid.

Percanthias TANAKA 1922 : P.PERCIF. (Percoid.) Serranid.

Percarina NORDMANN 1840 : P.PERCIF. (Percoid.) Percid.

Percichthys GIRARD 1854 : P.-PERCIF. (Percoid.) Serranid.

Percilia GIRARD 1854 : P.-PERCIF. (Percoid.) Serranid.

Percina HALDEMAN 1842 : P.-PERCIF. (Percoid.) Percid.

Percis SCOPOLI 1777 : P.-PERCIF. (Cottoid.) Agonid.

Percnopterus RAFINESQUE 1815 : syn. de Neophron SAVIGNY 1809.

Percnostola CABANIS et HEINE 1860: Av. PASSERIF. (Mesomyod.) Formicariid. (Formicariin.).

Percocypris CHU 1935 : P.-CYPRINIF. (Cyprinoid.) Cyprinid.

Percophis QUOY et GAIMARD 1824: P.-PERCIF. (Trachinoid.) Percophiid.

Percopsis AGASSIZ 1849 : P.-PERCOPSIF. Percopsid. 
Percosoma GILL 1861 : P.-PERCIF. (Percoid.) Serranid.

Percostoma AGASSIZ 1845 : P.PERCIF. (Percoid.) Sparid.

Percottus DYBOWSKI 1877: P. PERCIF. (Gobioid.) Eleotrid.

Perdicula HODGSON 1837: Av. PHASIANIF. Phasianid. (Phasianin.).

Perdix BRISSON 1760: Av. PHASIANIF. Phasianid. (Phasianin.).

Perealates : errat. pro Precalates RAMSAY et OGILBY 1887.

Pericrocotus BOIE 1826 : Av. PASSERIF. (Acromyod.) Campephagid.

Perigrammatolepis GEINITZ 1868 : P.-ZEIF. Zeid.

Perilampus Mc CLELLAND 1839 : P.-CYPRINIF. (Cyprinoid.) Cyprinid.

Periophthalmodon BLEEKER 1874 : s./g. de Periophthalmus SCHNEIDER 1801.

Periophthalmus SCHNEIDER 1801: P.-PERCIF. (Gobioid.) Periophthalmid.

Periops WAGLER 1830: syn. de Zamenis WAGLER 1830.

Periparus SELYS 1884: s./g. de Parus LINNE 1758.

Perisoreus BONAPARTE 1831: Av. PASSERIF. (Acromyod.) Corvid.

Perissias JORDAN et EVERMANN 1898 : P.-PLEURONECTIF. (Pleuronectoid.) Bothid.

Perissodus BOULENGER 1898 : P.PERCIF. (Percoid.) Cichlid.

Perissoglossa BAIRD 1864: Av. PASSERIF. (Acromyod.) Parulid.

Perissornis OBERHOLSER 1899 : Av. PASSERIF. (Acromyod.) Sturnid.

Perissospiza OBERHOLSER 1900 : Av. PASSERIF. (Acromyod.) Fringillid.

Perissotriccus OBERHOLSER 1903: Av. PASSERIF. (Mesomyod.) Tyrannid.

Peristedion de LACEPEDE 1802 : P.-PERCIF. (Scorpaenoid.) Triglid,
Peristera SWAINSON 1827:Av. COLUMBIF. Columbid. (Columbin.).

Peristethus KAUP 1858: syn. de Peristedion de LACEPEDE 1802.

Peristrominous WHITLEY 1952 : P. - PERCIF. (Scorpaenoid.) Scorpaenid.

Perkinsia EIGENMANN 1891: P.CLUPEIF. (Clupeoid.) Dussumierid.

Pernis CUVIER 1817: Av. FALCONIF. Falconid. (Milvin.).

Perochirus BOULENGER 1885 : R.SQUAMAT. (Saur.) Geckonid.

Perodactylus REINHARDT et LUTKIN 1862: R. $-S Q U A M A T$. (Saur.) Tejid.

Perodicticus BENNETT 1831: M.PRIMAT. (Lemuroid.) Lorisid.

Perodipus FITZINGER 1867: syn. de Dipodomys GRAY 1841.

Peroechinus FITZINGER 1866 : syn. d'Atelerix POMEL 1848.

Perognathus WIED 1839: M.-RODENT. Heteromyid. (Perognathin.).

Peromyscus GLOGER 1841 : M.-RODENT. Cric etid. (Hesperomyin.).

Peronymus PETERS 1868 : s./g. de Saccopteryx ILLIGER 1811.

Peropodum MULLER 1878: R.SQUAMAT. (Serpent.) Boid.

Peroptera GISTEL 1848: syn. de Cephaleutherus RAFINESQUE 1810.

Peropteryx PETERS $1867: \mathrm{s} . / \mathrm{g}$. de Saccopteryx ILLIGER 1811.

Peropus LAY et BENNETT 1839 : syn. de Blepsias CUVIER et VALENCIENNES 1829.

Peroryctes THOMAS 1906 : M.MARSUP. Peramelid.

Perosuchus COPE 1868: syn. de Jacaretinga SPIX 1825.

Perrunichthys SCHULTZ 1944 : P.CYPRINIF. (Siluroid.) Bagrid.

Perryena WHITLEY 1940 : P.-PERCIF. (Scorpaenoid.) Congiopid.

Persparsia PARR 1951: P.-CLUPEIF. (Alepocephaloid.) Alepocephalid. 
Pertica FOWLER 1904: P.-PERCIF. (Percozd) Leiognathid.

Perugia EIGENMANN et NORRIS 1901 : P.-CYPRINIF. (Siluroid.) Bagrid.

Pervagor WHITLEY 1930 : P.-PERCYF. (Balistoid.) Balistid. (ou Monacanthid.).

Pervicauda MATHEWS 1945: Av. ANATIF. Anatid. (Anatin.).

Pescadorichthys TOMIYAMA 1955 : P.-PERCIF. (Blennioid.) Blenniid.

Petacara BOHLKE 1959: P.-CYPRINIF. (Siluroid.) Bunocephalid.

Petalia GRAY 1838 : syn. de Nycteris GEOFFROY et CUVIER 1795.

Petalichthys REGAN 1904: P.-CYPRINODONTIF. Poeciliid.

Petalognathus DUMERIL et BI$B R O N$ 1854: R.-SQUA MAT. (Serpent.) Colubrid.

Petalosoma REGAN 1908: P.-CYPRINODONTIF. Poeciliid.

Petalurichthys REGAN 1912 : nom. nov. pro Petalosoma REGAN 1908.

Petasophora GRAY 1840 : Av. APODIF. Trochilid.

Petaurella MATSCHIE $1916: s . / g$. de Petaurus SHAW 1791.

Petaurillus THOMAS 1908 : M.-RODENT. (Sciuromorph.) Sciurid. (Petauristin.).

Petaurista LINK 1795: M.-RODENT. (Sciuromorph.) Sciurid. (Petauristin.).

Petaurista WATERHOUSE : syn. de Schoinobates LESSON 1842.

Petauroides THOMAS 1888: syn. de Schoinobates LESSON 1842.

Petaurus SHAW 1791 : M.-MARSUP. Phalangerid. (Phalangerin.).

Petenia GUNTHER 1862: P.-PERCIF. (Percoid.) Cichlid.

Petersialestes HOEDEMAN 1951: P.-CYPRINIF. (Characoid.) Characid.

Petersius HILGENDORF 1894 : P.CYPRINIF. (Characoid.) Characid.

Petinomys THOMAS 1908: M.-RODENT. (Sciuromorph.) Sciurid. (Petauristin.).
Petraites OGILBY 1885: P.-PERCIF. (Blennioid.) Clinid.

Petridium : errat. pro Petridium SCOPOLI 1777.

Petrocephalus MARCUSEN 1854: P.-CLUPEIF. (Mormyroid.) Mormyrid.

Petrochelidon CABANIS 1851: Av. PASSERIF. (Acromyod.) Hirundinid.

Petrochromis BOULENGER 1898 : P.-PERCIF. (Percoid.) Cichlid.

Petrocichla BLASIUS 1862 : syn. de Monticola BOIE 1822.

Petrodromus PETERS 1846: M.INSECTIV. Macroscelidid. (Macroscelidin.).

Petroeca AGASSIZ 1846: emend. pro Petroica SWAINSON 1829.

Petroica SWAINSON 1829: Av. PASSERIF. (Acromyod.) Muscicapid.

Petrogale GRAY 1837: M.-MARSUP. Macropodid. (Macropodin.).

Petrometopon GILL 1865 : P.-PERCIF. (Percoid.) Serranid.

Petromus SMITH 1831: M.-RODENT. (Hystricomorph.) Echimyid. (Petromyin.).

Petromys MARTINO 1935: syn. d'A podemus KAUP 1829.

Petromyscus THOMAS 1926: M.RODENT. (Myomorph.) Murid. (Dendromyin.).

Petromyzon LINNE 1758: Cycl. PETROMYZONIF. Petromyzonid.

Petronason SWAINSON 1839: P.PERCIF. (Labroid.) Scarid.

Petronia KAUP 1929: Av. PASSERIF. (Acromyod.) Ploceid. (Passerin.).

Petropedetes REICHENOW 1874 : Amph. ANOUR. Ranid.

Petrophassa GOULD 1841: Av. COLUMBIF. Columbid. (Columbin.).

Petrophila SWAINSON 1837: Av. PASSERIF. (Acromyod.) Turdid.

Petropseudes THOMAS 1923 : M.MARSUP. Phalangerid. (Phascolarctin.).

Petrorhynchus GRAY 1865 : syn. de Ziphius CUVIER 1823. 
Petrornis ROBERTS 1922: Av. PASSERIF. (Acromyod.) Turdid.

Petrosaurus BOULENGER 1885 : R.-SQUAMAT. (Saur.) Iguanid.

Petroschmidtia TARANETS et ANDRYASHEV 1924 : P.-PERCIF. (Blennioid.) Zoarcid.

Petroscirtes RUPPELL 1830 : P.PERCIF. (Blennioid.) Blenniid.

Petrotyx HELLER et SNODGRASS 1903 : P. - PERCIF. (Ophioid.) Brotulid.

Petrus SMITH 1938: P.-PERCIF. (Percoid.) Sparid.

Peucaea AUDUBON 1839: Av. PASSERIF. (Acromyod.) Fringillid.

Peucedramus C OUES 1875: Av. PASSERIF. (Acromyod.) Parulid.

Peyeria WEILER 1935 : P.-CYPRINIF. (Siluroid.) Bagrid.

Pezites CABANIS 1851: syn. de Sturnella VIEILLOT 1816.

Pezophaps STRICKLAND 1848: Av. COLUMBIF. Columbid. (Raphiin.) (éteint).

Pezoporus ILLIGER 1811: Av. PSITTACIF. Psittacid.

Pfrille JORDAN 1924 : P.-CYPRINIF. (Cyprinoid.) Cyprinid.

Phabotreron WALDEN 1875: errat. pro Phapitreron BONAPARTE 1854.

Phacellodomus REICHENBACH 1853: Av. PASSERIF. (Mesomyod.) Furnariid.

Phacellodromus B ONAPARTE 1854: errat. pro Phacellodomus REICHENBACH 1853.

Phacelodomus SALVIN 1882: errat. pro Phacellodomus REICHENBACH 1853.

Phaceloscenus RIDGWAY 1909: Av. PASSERIF. (Mesomyod.) Furnariid.

Phacochoerus CUVIER 1817: M.PARAX. (Suif.) Suid. (Suin.).

Phaenicircus SWAINSON 1832: Av. PASSERIF. (Mesomyod.) Cotingid.

Phaenicocercus BONAPARTE 1850: emend. pro Phaenicircus SWAINSON 1832 .
Phaenicophaeus STEPHENS 1915 : Av. CUCULLIF. Cucullid.

Phaenicophilus STRICKLAND 1851: Av. PASSERIF. (Acromyod.) Tanagrid.

Phaenicornis : errat. pro Phoenicornis BOIE 1827.

Phaenodon LOWE 1850 : P.-CLUPEIF. (Stomiatoid.) Astronesthid.

Phaenomonas MYERS 1941 : P.$A N G U I L L I F . \quad$ (Congroid.) Ophichthyid.

Phaenomys THOMAS 1917 : M.RODENT. (Myomorph.) Cricetid. (Hesperomyin.).

Phaenopogon HERRE 1935 : syn. de Cirrhigaleus TANAKA 1912.

Phaenorhina GRAY 1859: Av. COLUMBIF. Columbid. (Treronin.).

Phaenostictus RIDGWAY 1909: Av. PASSERIF. (Mesomyod.) Formicariid.

Phaeochroa GOULD 1861: Av. APODIF. Trochilid.

Phaeolaima REICHENBACH 1854 : Av. APODIF. Trochilid.

Phaeomyias BERLEPSCH et HARTERT 1902: Av. PASSERIF. (Mesomyod.) Tyrannid.

Phaeoprogne BAIRD 1865: Av. PASSERIF. (Acromyod.) Hirundinid.

Phaeoptila GOULD 1861 : Av. APODIF. Trochilid.

Phaeornis SCLATER 1869: Av. PASSERIF. (Acromyod.) Turdid. (Myadestin.).

Phaeothlypis TODD 1929 : Av. PASSERIF. (Acromyod.) Parulid.

Phaeotriccus RIDGWAY 1907: Av. PASSERIF. (Mesomyod.) Tyrannid.

Phaëton LINNE 1758: Av. PELECANIF. Phaëtontid.

Phaetonichthys BLEEKER 1876 : P.-PERCIF. (Percoid.) Serranid.

Phaetornis (SWAINSON 1827) CUVIER 1829: Av, APODIF. Trochilid.

Phaëtusa WAGLER 1832: Av. LARIF. Larid. (Sternin.). 
Phago GUNTHER 1865 : P.-CYPRINIF. (Characoid.) Characid.

Phagoborus MYERS 1924: P.-CYPRINIF. (Characoid.) Characid.

Phagorus Mc CLELLAND 1845 : P.CYPRINIF. (Siluroid.) Clariid.

Phagrus MARCUSEN 1864 (nec HECKEL 1849) : P.CLUPEIF. (Mormyroid.) Mormyrid.

Phainopepla BAIRD 1858: Av. PASSERIF. (Acromyod.) Bombycillid.

Phaiolaima REICHENBACH 1854: syn. de Heliodoxa GOULD 1850.

Phaiomys BLYTH 1863: M.-RODENT. (Myomorph.) Microtid.

Phalacrocorax BRISSON 1760: Av. PELECANIF. Phalacrocoracid. (Phalacrocoracin.).

Phalacromacrurus : P.-GADIF. Macrurid.

Phalacronotus BLEEKER 1858 : P.CYPRINIF. (Siluroid.) Silurid.

Phalacrotreron BONAPARTE 1854: Av. C OL UMBIF. Columbid. (Treronin.).

Phalaenoptilus RIDGWAY 1880 : Av. CAPRIMULGIF. Caprimulgid. (Caprimulgin.).

Phalanger STORR 1780: M.-MARSUP. Phalangerid. (Phalangerin.).

Phalangista GEOFFROY-ST-HILAI$R E$ et CUVIER 1795: syn. de Phalanger STORR 1780.

Phalangistes PALLAS 1814: P.PERCIF. (Cottoid.) Agonid.

Phalaropus BRISSON 1760: Av. CHARADRIIF. Charadriid. (Phalaropin.).

Phalcobaenus d'ORBIGNY 1835 : Av. FALCONIF, Falconid.

Phalerebus WHITLEY 1929: P.PERCIF. (Labroid.) Pomacentrid.

Phaleris TEMMINCK 1820 : Av. ALCIF. Alcid. (Fraterculin.).

Phallichthys HUBBS 1925 : P.-CYPRINODONTIF. Poeciliid.

Phalloceros EIGENMANN 1907 : P.CYPRINODONTIF. Goodeid.

Phallocottus SCHULTZ 1938: P.DERCIF. (Cottoid.) Cottid.
Phalloptychus EIGENMANN 1907 : P. - CYPRINODONTIF. Poeciliid.

Phallostethus REGAN 1913: P.PHALLOSTETHIF, Phallostethid.

Phallotorvnus HENN 1916 : P.-CYPRINODONTIF. Poeciliid.

Phanagoniates : voir Phenagoniates EIGENMANN et WILSON 1914.

Phaner GRAY 1872: M.-PRIMAT. (Lemuroid.) Lemurid. (Cheirogalein.).

Phanerobranchus COCCO 1846 : syn. de Myristibranchus WHITLEY 1939.

Phanerobranchus LEUCKART 1821: Amph.

Phanerocephalus GRATZIANOV 1906: P. - RAJIF. (Dasyatoid.) Brachyopterid.

Phanerodon GI R A R D 1854: P. PERCIF. (Labroid.) Embiotocid.

Phanerotis BOULENGER 1890 : Amph. ANOUR. Leptodactylid.

Phanerotokeus DUNCKER 1940: P.-SYNGNATHIF. Syngnathid.

Phapitreron BONAPARTE 1854: Av. C OLU MBIF. Columbid. (Treronin.).

Phaps SELBY 1835: Av. COLUMBIF. Columbid. (Columbin.).

Pharomachrus de la LLAVE 1832 : Av. TROGONIF. Trogonid.

Pharopteryx RUPPELL 1828: P.PERCIF. (Percoid.) Plesiopid.

Pharotis THOMAS 1914: s./g. de Nyctophilus LEACH 1821.

Phascogale TEMMINCK 1824: M.MARSUP. Dasyurid. (Phascogalin.).

Phascolarctos de BLAINVILLE 1816: M.-MARSUP. Phalangerid. (Phascolarctin.).

Phascolarctus BROOKES 1828 : errat. pro Phascolarctos de BLAIN VILLE 1816.

Phascologale LENZ 1831: errat. pro Phascogale TEMMINCK 1824.

Phascolomys DUMERIL 1806 : syn. de Vombatus GEOFFROY-ST. HILAIRE 1803. 
Phascolosorex MATSCHIE 1916 : M.-MARSUP. Dasyurid. (Dasyurin.).

Phasianus LINNE 1758: Av. PHASIANIF. Phasianid. (Phasianin.).

Phasidus CASSIN 1856: Av. PHASIANIF. Phasianid. (Munidin.).

Phasmatocottus BOLIN 1936: P.PERCIF. (Cottoid.) Cottid.

Phasmatostoma MYERS 1940 : P.MONOGNATHIF. Monognathid.

Phasmichthys JORDAN et HUBBS 1925: P. - HOLOCEPH. Callorhynchid.

Phasmostoma : errat. pro Phasmatostoma MYERS 1940.

Phatages SUNDEWALL 1843 : syn. de Manis LINNE 1758.

Phataginus RAFINESQUE 1815: syn. de Manis LINNE 1758.

Phaulomys THOMAS 1905 : syn. de Clethrionomys TILESIUS 1850.

Phedina BONAPARTE 1855: Av. PASSERIF. (Acromyod.) Hirundinid.

Phegornis GRAY 1847: Av. CHARADRIIF. Charadriid. (Scolopacin.).

Phelsuma GRAY 1825: R.-SQUAMAT. (Saur.) Geckonid.

Phenacobius COPE 1867: P.-CYPRINIF. (Cyprinoid.) Cyprinid.

Phenacobrycon EIGENMANN 1922: P.-CYPRINIF. (Characoid.) Characid.

Phenacogaster EIGENMANN 1916 : P.-CY PR I NIF. (Characoid.) Characid.

Phenacogrammus EIGENMANN 1907: P.-CYPRINIF. (Characoid.) Characid.

Phenacomys MERRIAM 1889: M.RODENT. (Myomorph.) Microtid.

Phenascosaurus BARBOUR 1920 : R.-SQUAMAT. (Saur.) Lacertid.

Phenacoscorpius FOWLER 1938: P. - PERCIF. (Scorpaenoid.) Scorpaenid.

Phenacostethus MYERS 1928: P.PHALLOSTETHIF. Phallostethid.

Phenagoniates E I G ENM A N N et
WILSON 1914 : P.-CYPRINIF. (Characoid.) Characid.

Pherallodiscus BRIGGS 1955 : P.PERCIF. (Gobiesocoid.) Gobiesocid.

Pherallodus B R I G GS 1955 : P.PERCIF. (Gobiesocoid.) Gobiesocid.

Pherocraspedon MATHEWS 1931 : Av. PASSERIF. (Acromyod.) Paradiseid. (Paradisein.).

Pheucticus REICHENBACH 1850 : Av. PASSERIF. (Acromyod.) Fringillid. (Coccothrausthin.).

Pheugopedius CABANIS 1851 : syn. de Thriothorus VIEILLOT 1816.

Phibalura VIEILLOT 1816: Av. PASSERIF. (Mesomyod.) Cotingid.

Phigyps AGASSIZ 1842: Av. PSITTACIF. Psittacid.

Philacte BANNISTER 1870: Av. ANATIF. Anatid. (Anserin.).

Philetairus SMITH 1837 : Av. PASSERIF. (Acromyod.) Ploceid. (Passerin.).

Philander B RIS S ON 1762: M.MARSUP. Didelphid.

Philantomba BLYTH 1840 : M.-PARAX. Bovid. (Cephalophin.).

Philautus GIS T E L 1848: Amph. ANOUR. Racophorid.

Philemon VIEILLOT 1816: Av. PASSERIF. (Acromyod.) Meliphagid.

Philemonopsis SALVADORI 1880: Av. PASSERIF. (Acromyod.) Meliphagid.

Philentoma EYTON 1845: Av. PASSERIF. (Acromyod.) Muscicapid.

Philepitta GEOFFROY - ST - HILAIRE 1838:Av. PASSERIF. (Mesomyod.) Philepittid.

Philesturnus GEOFFROY-ST-HILAIRE 1832: Av. PASSERIF. (Acromyod.) Sturnid. (Graculin.).

Philetairus SMITH 1837: Av. PASSERIF. (Acromyod.) Ploceid. $\cdot\left(\cdot u ! . l \partial s s D_{d}\right)$

Philetor THOMAS 1902: M.-CHIROPT. (Microchiropt.) Vespertilionid. (Vespertilionin.). 
Philipinnia : Av. PASSERIF. (Acromyod.) Nectariniid.

Philippinopterus TAYLOR 1934: syn. de Tadarida RAFINESQUE 1814.

Philippsichthys WHITLEY 1937 : P.-PERCIF. (Blennioid.) Clinid.

Philochortus MATSCHIE 1893 : R.SQUAMAT. (Saur.) Lacertid.

Philodice MULSANT et VERREAUX 1866: Av. APODIF. Trochilid.

Philodryas WAGLER 1830: R.SQUAMAT. (Serpent.) Boigid.

Philohela GRAY 1841: Av. CHARADRIIF. Charadriid. (Scolopacin.).

Philomachus GRAY 1840: Av. CHARADRIIF. Charadriid. (Scolopacin.).

Philomela LINK 1806 : syn. de Luscinia KAUP 1829.

Philomyias : errat. pro Phyllomyias CABANIS et HEINE 1860.

Philoria SPENCER 1901: Amph. ANOUR. Leptodactylid.

Philortyx GOULD 1846: Av. PHASIANIF. Phasianid. (Phasianin.).

Philothamnus SMITH 1847 : R.SQUAMAT. (Serpent.) Colubrid.

Philydor SPIX 1824: Av, PASSERIF. (Mesomyod.) Furnariid.

Philypnodon BLEEKER 1874 : P.PERCIF. (Gobioid.) Eleotrid.

Philypnoides BLEEKER 1849: P. PERCIF. (Ophiocephaloid.) Ophiocephalid.

Philypnus CUVIER et VALENCIENNES 1837: P.-PERCIF, (Gobioid.) Eleotrid.

Phimosus WAGLER 1832: Av. ARDEIF. Plataleid. (Threskiornithin.).

Phlegoenas REICHENBACH 1852 : Av. COL UMBIF. Columbid. (Columbin.).

Phlegopsis REICHENBACH 1852: Av. PASSERIF. (Mesomyod.) Formicariid.

Phloeoceastes CABANIS 1862: Av. PICIF. Picid.

Phloeocryptes CABANIS et HEINE 1859: Av. PASSERIF. (Mesomyod.) Furnariid.
Phleomys WATERHOUSE 1839: M.-RODENT. (Myomorph.) Murid. (Murin.).

Phloeotomus CABANIS et HEINE 1863: Av. PICIF. Picid. (Picin.).

Phlogophilus GOULD 1860: Av. APODIF. Trochilid.

Phlogoplectes WOLTERS 1943 : s./g. d'Euplectes SWAINSON 1829 .

Phlogopsis SCLATER 1858 : errat. pro Phlegopsis REICHENBACH 1850.

Phlogothraupis SCLATER et SALVIN 1873: Av. PAS SERIF. (Acromyod.) Tanagrid.

Phlyctimantis : Amph. ANOUR. Racophorid.

Phobetor KROYER 1844 : P.-PERCIF. (Cottoid.) Cottid.

Phoca LINNE 1758: M.-PINNIP. Phocid. (Phocin.).

Phocaegadus JENSEN : syn. d'Arctogadus DRJAGIN 1932.

Phocaena CUVIER 1817: M.-CETAC. (Odontocet.) Phocaenid.

Phocoena CUVIER 1816: syn. de Phocaena CUVIER 1817.

Phocarctos PETERS 1866 : M.PINNIP. Phocid. (Phocin.).

Phocena GRAY 1821 : syn. de Phocaena CUVIER 1817.

Phocoenoides ANDREWS 1911: $s . / g$. de Phocaena CUVIER 1817.

Phodilus GEOFFROY-ST-HILAIRE 1830 : Av. STRIGIF. Strigid. (Tytonin.).

Phodopus MILLER 1910: M.-RODENT. (Myomorph.) Cricetid. (Cricetin.).

Phodotes MILLER 1906: s./g. de Natalus GRAY 1838.

Phoeaythia DELACOUR 1937 : Av. ANATIF. Anatid. (Anatin.).

Phoebastria REICHENBACH 1852 : Av. PROCELLARIF. Diomedeid.

Phoebetria REICHENBACH 1852 : Av. PROCELLARIF. Diomedeid.

Phoenicercus STRICKLAND 1841: emend. pro Phoenicircus SWAINSON 1832. 
Phoenicircus SWAINSON 1832 : Av. PASSERIF. (Mesomyod.) Cotingid.

Phoenicocercus CABANIS 1847: emend. pro Phoenicircus SWAINSON 1832.

Phoenicocichla CABANIS et HEINE 1860: Av. PASSERIF. (Mesomyod.) Pittid.

Phoeniconaias GRAY 1869: Av. ANATIF. (Phoenicopterif.) Phaenicopterid.

Phoenicoparrus BONAPARTE 1856: Av. ANATIF. (Phoenicopterif.) Phaenicopterid.

Phoenicophaes CABANIS et HEINE 1863: emend. pro Phaenicophaeus STEPHENS 1815.

Phoenicophaeus : vide Phaenicophaeus STEPHENS 1815.

Phoenicophaius GRAY 1849 : emend. pro Phaenicophaeus STEPHENS 1815.

Phoenicophanes NITZSCH 1829 : emend. pro Phaenicophaens STEPHENS 1815.

Phoenicophaus VIEILLOT 1816: emend. pro Phaenicophaeus STEPHENS 1815.

Phoenicophous SCLATER 1854: emend. pro Phaenicophaeus STEPHENS 1815.

Phoenicophilus : vide Phaenicophilus STRICKLAND 1851.

Phoenicopterus LINNE 1758: Av. ANATIF. (Phoenicopterif.) Phaenicopterid.

Phoenicornis BOIE 1827: Av. PASSERIF. (Acromyod.) Tanagrid.

Phoenicothraupis CABANIS 1851 : Av. PASSERIF. (Acromyod.) Tanagrid.

Phoeniculus JAROCKI 1821: Av. CORACIADIF. Upupid. (Phoeniculin.).

Phoenicuroides ROBERTS 1922 : Av. PASSERIF. (Acromyod.) Turdid. (Phoenicurin.).

Phoenicurus FORSTER 1817: Av. PASSERIF. (Acromyod.) Turdid. (Phoenicurin.).

Phoeoaythia : vide Phoeaythia DELACOUR 1937.

Phoetornis FERUSSAC 1831 : syn. de Phaethornis SWAINSON 1827 (vide Phaetornis CUVIER).

Pholidauges CABANIS 1851: Av PASSERIF. (Acromyod.) Sturnid. (Graculin.).

Pholidichthys BLEEKER 1856 : P.PERCIF. (Ophidioid.) Pholidichthyid.

Pholidobolus PETERS 1862: R.SQUAMAT. (Saur.) Tejid.

Pholidornis HARTLAUB 1857: Av. PASSERIF. (Acromyod.) Dicaeid.

Pholidotus BRISSON 1762 : syn. de Manis LINNE 1758.

Pholis GRONOV 1760 : P.-PERCIF. (Blennioid.) Pholid.

Phoniscus MILLER 1905: s./g. de Kerivoula GRAY 1842.

Phonygammus LESSON et GARNOT 1826: Av. PASSERIF. (Acromyod.) Paradiseid. (Paradisein.).

Phormoplectes REICHENOW 1903: Av. PASSERIF. (Acromyod.) Ploceid.

Photichthys HUTTON 1873: P.CLUPEIF. (Stomiat.) Gonostomid.

Photoblepharon WEBER 1902 : P.PERCIF. (Percoid.) Carangid.

Photocorynus RE GAN 1925: P. LOPHIIF. (Ceratioid.) Linophrynid.

Photogenis COPE 1869 : P.-CYPRIN7F. (Cyprinoid.) Cyprinid.

Photonectes GUNTHER 1887 : P.CLUPEIF. (Stomiatoid.) Melanostomiatid.

Photonectoides KOEFOED 1956 : P. - CL UPE I F. (Stomiatoid.) Melanostomiatid.

Photonectops CHAPMAN 1939 : P.CLUPEIF. (Stomiatoid.) Melanostomiatid.

Photostomias COLLETT 1889 : P.CLUPEIF. (Stomiatoid.) Malacosteid.

Photostylus BEEBE 1933 : P.-CLUPEIF. (Alepocephaloid.) Alepocephalid.

Phoxargyrea FOWLER 1903 : P.MUGILIF. Atherinid.

Phoxinellus HECKEL 1843 : P.-CYPRINIF. (Cyprinoid.) Cyprinid. 
Phoxinus HERMANN 1804 : P.-CYPRINIF. (Cyprinoid.) Cyprinid.

Phoxinopsis REGAN 1907 : P.-CYPRINIF. (Characoid.) Characid.

Phoxiscus OSHIMA 1919 : P.-CYPRINIF. (Cyprinoid.) Cyprinid.

Phoxonopsis INNES 1938 : syn. de Spintherobolus EIGENMANN 1911.

Phoxophrys HUBRECHT 1881 : R.SQUAMAT. (Saur.) Agamid.

Phoyx STEJNEGER 1887 : Av. ARDEIF. Ardeid.

Phractocenhalus AGASSIZ 1829: P.-CYPRINIF. (Siluroid.) Bagrid.

Phractolaemus BOULENGER 1901: P. - CLUPEIF. (Channoid.) Phractolaemid.

Phractura BOULENGER 1900 : P.AULOSTOMIF. Amphisiliid.

Phragmaticola JERDON 1844: Av. PASSERIF. (Acromyod.) Sylviid.

Phreatichthys VINCIGUERRA 1924: P. - CYPRINIF. (Cyprinoid.) Cyprinid.

Phreatobius GOELDI 1905 : P.-CYPRINIF. (Siluroid.) Trichomycterid.

Phrenotriccus RICHEMOND 1927 : Av. PASSERIF. (Mesomyod.) Tyrannid.

Phrydops BOULENGER 1905 : syn. d'Amastridium COPE 1860.

Phrygilus CABANIS 1844: Av. PASSERIF. (Acromyod.) Fringillid. (Emberizin.).

Phrynanodus AHL 1933: Amph. ANOUR. Leptodactylid.

Phrynella BOULENGER 1887 : syn. de Kaloula GRAY 1831.

Phrynelox WHITLEY 1931 : P.-LO PHIIF. (Antennarioid.) Antennariid.

Phrynichthys AGASSIZ 1846: P.LOPHIIF. (Ceratioid.) Himantolophid.

Phrynichthys PIETSCHMANN 1926: syn. de Bufoceratias WHITLEY 1931.

Phryniscus WIEGMANN 1835 : syn. d'Atelopus DUMERIL et BIBRON 1841.
Phrynixalus BOETTEGER 1894: Amph. ANOUR. Microhylid.

Phrynobatrachus GUNTHER 1862: Amph. ANOUR. Ranid.

Phrynocara PETERS 1883 : Amph. ANOUR. Microhylid.

Phrynocephalus KAUP 1827: R.SQUAMAT. (Saur.) Agamid.

Phrynoderma BOULENGER 1893: syn. de Rhacophorus KUHL et von HASSELT 1822.

Phrynodon PARKER 1935: Amph. ANOUR. Ranid.

Phrynoglossus PETERS 1867: Amph. ANOUR. Ranid.

Phrynohyas FITZINGER 1843 : Amph. ANOUR. Hylid.

Phrynomantis PETERS 1867: Amph. ANOUR. Microhylid.

Phrynomerus NOBLE 1926 : Amph. ANOUR. Microhylid.

Phrynonax COPE 1862: syn. de Synchalinus COPE 1894.

Phrynops WAGLER 1830 : syn. de Hydraspis BELL 1828.

Phrynopsis PFEFFER 1893 : Amph. ANOUR. Ranid.

Phrynorhombus GUNTHER 1862 : P. - PLEURONECTIF. (Pleuronectoid.) Bothid.

Phrynosaura WERNER 1907: R.SQUAMAT. (Saur.) Iguanid.

Phrynosoma WIEGMANN 1828 : R.SQUAMAT. (Saur.) Iguanid.

Phtheirichthys GILL 1862 : P.-PERCIF. (Echenoid.) Echeneid.

Phycis WALBAUM 1792 : P.-GADIF. Gadid.

Phycodurus GILL 1896 : P.-SYNGNATHIF. Syngnathid.

Phyllastrephus SWAINSON 1832 : Av. PASSERIF. (Acromyod.) Pycnonotid.

Phyllergates SHARPE 1883: Av. PASSERIF. (Acromyod.) Timaliid.

Phyllichthys Mc CULLOCH 1916 : P.-PLEURONECTIF. (Soleoid.) Soleid.

Phyllobates SHARPE 1883: Amph. ANOUR. Brachycephalid.

Phyllodactylus GRAY 1828: R.SQUAMAT. (Saur.) Geckonid. 
Phylloderma PETERS 1865: M.CHIROPT. (Microchiropt.) Phyllodermatid. (Phyllodermatin.).

Phyllodromus ESPADA 1875 : Amph. ANOUR. Ranid.

Phyllodryas : errat. pro Philodryas WAGLER 1830.

Phyllogephyra : errat. pro Phylogephyra BOULENGER 1898.

Phyllogramma PELLEGRIN 1934: P.-ANGUILLIF. (Congroid.) Congrid.

Phyllolais HARTLAUB 1881: Av. PASSERIF. (Acromyod.) Timaliid.

Phyllomedusa WAGLER 1830 : Amph. ANOUR. Phyllomednsid.

Phyllomyias CABANIS et HEINE 1860: Av. PASSERIF. (Mesomyod.) Tyrannid. (Spizornithin.).

Phyllomys LUND 1841: syn. d'Echimys CUVIER 1809.

Phyllonemus BOULENGER 1906 : P.-CYPRINIF. (Siluroid.) Bagrid.

Phyllonycteris GUNDLACH 1860 : M. -CHIROPT. (Microchiropt.) Phyllostomatid. (Phyllonycterin.).

Phyllooecia BERTONI 1901 : syn. de Leptotriccus CABANIS et HEINE 1860.

Phyllopezus PET ER S 1877 : R.SQUAMAT. (Saur.) Geckonid.

Phyllophichthys GOSLINE 1952: P.-ANGUILLIF. (Congroid.) Ophichthyid.

Phyllops PETERS 1876: s./g. de Stenoderma OKEN 1816.

Phyllopseute MEYER 1815: syn. de Phylloscopus BOIE 1826.

Phyllopteryx SWAINSON 1839 : P.SYNGNATHIF. Syngnathid.

Phyllorhina BONAPARTE 1837: syn. de Hipposideros GRAY 1931.

Phyllorhynchus STEJNEGER 1890: R.-SQUAMAT. (Serpent.) Colubrid.

Phyllornis TEMMINCK 1829: Av. PASSERIF. (Acromyod.) Pycnonotid.
Phylloscartes CABANIS et HEINE 1860: Av. PASSERIF. (Mesomyod.) Tyrannid. (Platyrhynchid.).

Phylloscopus BOIE 1826 : Av. PASSERIF. (Acromyod.) Sylviid.

Phyllosphus : Av. PASSERIF. (Acromyod.) Pycnonotid.

Phyllostoma CUVIER 1800 : syn. de Phyllostomus de LACEPEDE 1799.

Phyllostomus de LACEPEDE 1899 : M.-CHIROPT. (Microchiropt.) Phyllostomatid. (Phyllostomatin.).

Phyllostrephus AGASSIZ 1846 : emend. pro Phyllastrephus SWAINSON 1832.

Phyllotis GRAY 1867 : syn. de Rhinolophus de LACEPEDE 1799.

Phyllotis WATERHOUSE 1837 : M.-RODENT. (Myomorph.) Cricetid. (Hesperomyin.).

Phyllurus OKEN 1817: R.-SQUAMAT. (Saur.) Geckonid.

Phyllogephyra BOULENGER 1898 : P.-CLUPEIF. (Salmonoid.) Salmonid.

Phymaturus GRAVENHORST 1838: R.-SQUAMAT. (Saur.) Iguanid.

Physailia BOULENGER 1901: P.CYPRINIF, (Siluroid.) Schilbeid.

Physalaemus FITZINGER 1826: Amph. ANOUR. Leptodactylid.

Physalus de LACEPEDE 1804: syn. de Physeter LINNE 1758.

Physeter LINNE 1758: M.-CETAC. (Odontocet.) Physeterid. (Physeterin.).

Physiculus KAUP 1858 : P.-GADIF. Morid.

Physignathus CUVIER 1829: R.SQUAMAT. (Saur.) Agamid.

Physocorax BONAPARTE 1853: Av. PASSERIF. (Acromyod.) Corvid.

Physodon MULLER et HENLE 1841: P.-GALEIF. (Carcharhinoid.) Carcharhinid.

Physopyxis COPE 1871: P.-CYPRINIF. (Siluroid.) Doradid.

Phytichthys JORDAN 1923: P.PERCIF. (Blennioid.) Pholid. 
Phytotoma MOLINA 1782: Av. PASSERIF. (Mesomyod.) Phytotomid.

Piabarchus MYERS 1928: P.-CYPRINIF. (Characoid.) Characid.

Piabina REINHARDT 1866 : P.-CYPRINIF. (Characoid.) Characid.

Piabuca MULLER 1843: emend. pro Piabucus OKEN 1817.

Piabucidium MYERS 1929 : P.-CYPRINIF. (Characoid.) Characid.

Piabucina CUVIER et VALENCIENNES 1849 : P.-CYPRINIF. (Characoid.) Characid.

Piabucus OKEN 1817 : P.-CYPRINIF. (Characoid.) Characid.

Piaractus EIGENMANN 1905: P.CYPRINIF. (Characoid.) Characid.

Piaya LESSON 1830 : Av. CUCULLIF. Cucullid.

Pica BRISSON 1760: Av. PASSERIF. (Acromyod.) Corvid.

Picathartes LESSON 1828: Av PASSERIF. (Acromyod.) Sturnid. (Picathartin.).

Picicorvus BONAPARTE 1850 : syn. de Nucifraga BRISSON 1760.

Picoides de LACEPEDE 1760: Av. PICIF. Picid. (Picin.).

Picolaptes LESSON 1830 : syn. de Lepidocolaptes REICHEN$B A C H 1853$.

Pictiblennius WHITLEY 1930 : P.PERCIF. (Blennioid.) Blenniid.

Pictilabrus GILL 1891 : P.-PERCIF. (Laboid.) Labrid.

Piculus SPIX 1824: Av. PICIF. Picid. (Picin.).

Picumnoides BONAPARTE 1854: Av. PICIF. Picid. (Picumnin.).

Picumnus TEMMINCK 1825: Av. PICIF. Picid. (Picumnin.).

Picus LINNE 1758: Av. PICIF. Picid. (Picin.).

Piezorhina LAFRESNAYE 1843 : Av. PASSERIF. (Acromyod.) Fringillid. (Pitylin.).

Piezorhynchus GOULD 1841: Av. PASSERIF. (Acromyod.) Muscicapid.

Piezormona MATHEWS 1925 : syn. de Monarcha VIGORS et HORSFIELD 1826.

Pigus BONAPARTE 1845: P.-CYPRINIF. (Cyprinoid.) Cyprinid.

Pika de LACEPEDE 1799: s./g. d'Ochotona LINK 1795.

Pikea STEINDACHNER 1875 : P.PERCIF. (Percoid.) Serranid.

Pileoma DEKAY 1842 : P.-PERCIF. (Percoid.) Etheostomid.

Pilherodius REICHENBACH 1852 : Av. ARDEIF. Ardeid.

Pilicolobus ROCHEBRUNE 1886: syn. de Colobus ILLIGER 1811.

Pilodictis : vide Pylodictis RAFINESQUE 1819.

Pilorhinus CABAN IS 1851: Av. PASSERIF. (Acromyod.) Sturnid. (Graculin.).

Pimelepterus de LACEPEDE 1802: P.-PERCIF. (Percoid.) Kyphosid.

Pimelodella EIGENMANN 1888 : P.-CYPRINIF. (Siluroid.) Bagrid.

Pimelodus de LACEPEDE 1803: P.-CYPRINIF. (Siluroid.) Bagrid.

Pimelometopon GILL 1864: P. PERCIF. (Labroid.) Labrid.

Pimelonotus GILL 1858: P.-CYPRINIF. (Siluroid.) Bagrid.

Pimelotropis GILL 1859: P.-CYPRINIF. (Siluroid.) Bagrid.

Pimephales RAFINESQUE 1820 : P.-CYPRINIF. (Cyprinoid.) Cyprinid.

Pinalia GRAY 1838 : syn. de Neomys GRAY 1873.

Pinarochroa SUNDEVALL 1872 : Av. PASSERIF. (Acromyod.) Timaliid.

Pinarocichla SHARPE 1881: Av. PASSERIF. (Acromyod.) Pycnonotid.

Pinarocorys SHELLEY 1902: Av. PASSERIF. (Acromyod.) Alaudid.

Pinarolestes SHARPE 1877: syn. de Clytorhynchus ELLIOT 1870 .

Pinaroloxias SHARPE 1885: Av. PASSERIF. (Acromyod.) Fringillid. (Geospizin.). 
Pinarornis SHARPE 1876: Av. PASSERIF. (Acromyod.) Timaliid.

Pingalla WHITLEY 1954: P.-PERCIF. (Percoid.) Serranid.

Pingia CHANG 1935: Amph. UROD. Salamandrid.

Pinguilabrum : P.-PERCIF. (Percoid.) Serranid.

Pinguinus BONNATERRE 1790: Av. ALCIF. Alcid. (Alcin.).

Pinguipes CUVIER et VALENCIENNES 1829: P.-PERCIF. (Trachinoid.) Pinguipedid.

Pinicola VIEILLOT 1807 : Av. PASSERIF. (Acromyod.) Fringillid. (Fringillin.).

Pinirampus BLEEKER 1857: P.CYPRINIF. (Siluroid.) Bagrid.

Pinjalo BLEEKER 1875: P.-PERCIF. (Percoid.) Lutjanid.

Pintoichthys FOWLER 1954: P.PERCIF. (Percoid.) Cichlid.

Pionias FINSCH 1867 : syn. de Pionus WAGLER 1832.

Pionites HEINE 1890 : Av, PSITTACIF. Psittacid. (emend. pro Caica LESSON 1830).

Pionopsitta BONAPARTE 1854: Av. PSITTACIF. Psittacid.

Pionopsittacus SUNDEVALL 1872 : emend. pro Pionopsitta BONAPARTE 1854.

Pionus WAGLER 1832: Av. PSITTACIF. Psittacid.

Pipa LA URENTI 1868: Amph. ANOUR. (Aglos.) Pipadid.

Pipastes KAUP 1829: s./g. d'Anthus BECHSTEIN 1805.

Pipetella WHITLEY: syn. de Nigracus WHITLEY 1953.

Pipettella HA E C K EL 1887 : P.SYNGNATHIF. Syngnathid.

Pipidonia SMITH 1931 : P.-PERCIF. (Gobioid.) Gobiid.

Pipile B ONAPARTE 1856: Av. PHASIANIF. Cracid.

Pipilo VIEILLOT 1816: Av. PASSERIF. (Acromyod.) Tanagrid.

Pipilopsis BONAPARTE $1850: \mathrm{s} . / \mathrm{g}$. de Buarremon BONAPARTE 1850.

Pinistrellus KAUP 1829: M.-CHIROPT. (Microchiropt.) Vespertilionid. (Vespertilionin.).
Pipra LINNE 1766 : Av. PASSERIF. (Mesomyod.) Piprid.

Pipraeidea SWAINSON 1827: Av. PASSERIF. (Acromyod.) Tanagrid.

Pipreola SWAINSON 1837: Av. PASSERIF. (Mesomyod.) Cotingid.

Pipridea SCLATER 1857 : emend. pro Pipraeidea SWAINSON 1827.

Piprisoma BLYTH 1844 : Av. PASSERIF. (Acromyod.) Dicaeid.

Piprites CABANIS 1847: Av. PASSERIF. (Mesomyod.) Piprid.

Pipromorpha BONAPARTE 1854: Av. PASSERIF. (Mesomyod.) Tyrannid.

Piramutana BLEEKER 1863: P.CYPRINIF. (Siluroid.) Bagrid.

Piranga VIEILLOT 1807 : Av. PASSERIF. (Acromyod.) Tanagrid.

Pirarara SPIX 1829 : P.-CYPRINIF. (Siluroid.) Bagrid.

Piratinga BLEEKER 1863 : P.-CYPRINIF. (Siluroid.) Bagrid.

Pirellinus WHITLEY 1928 : syn. de Carapus RAFINESQUE 1810.

Pirene GISTEL 1848 : emend. pro Dascyllus CUVIER 1829.

Pirenestes SWAINSON 1837 : vide Pyrenestes SWAINSON 1837.

Pirinampus GUNTHER 1864: errat. pro Pinirampus BLEEKER 1857.

Pisciregia ABBUTT 1899 : P.-MUGILIF. Atherinid.

Pisobia BILLBERG 1828 : Av. CHARADRIIF. Charadriid. (Scolopacin.).

Pisoodonophis KA UP 1860: P.ANGUILLIF. (Congroid.) Ophichthyid.

Pisorhina KAUP 1848: syn. de Otus PENNANT 1769.

Pitanga : errat. pro Pitangus SWAINSON 1827.

Pintangus SWAINSON 1827: Av. PASSERIF. (Mesomyod.) Tyrannid. (Tyrannin.).

Pithecheir CUVIER 1838: M.-RODENT. (Myomorph.) Murid. (Murin.).

Pithechir JENTINK 1892: errat. pro Pithecheir CUVIER 1838. 
Pithecia DESMAREST 1804: M.PRIMAT. (Simioid.) Cebid. (Pithecin.).

Pithecocharax FOWLER 1906 : P.CYPRINIF. (Characoid.) Characid.

Pithecomyzon FOWLER 1913 : P.CYPRINIF. (Cyprinoid.) Catostomid.

Pithecophaga OGILVIE-GRANT 1896: Av. FALCONIF. Falconid. (Aquilin.).

Pithecopsis DUMERIL et BIBRON 1840 : syn. de Telmatobius WIEGMANN 1835.

Pithecopsis GUNTHER: syn. de Grypiscus COPE 1867.

Pithecopus COPE 1866: s./g. de Phyllomedusa WAGLER 1830.

Pithecus ELLIOT 1913: syn. de Macaca de LACEPEDE 1799.

Pithes BURNETT 1828: syn. de Macaca de LACEPEDE 1799.

Pithys VIEILLOT 1818: Av. PASSERIF. (Mesomyod.) Formicariid. (Formicariin.).

Pitohui LESSON 1831: Av. PASSERIF. (Acromyod.) Pachycephalid.

Pitta VIEILLOT 1816 : Av. PASSERIF. (Mesomyod.) Pittid.

Pittasoma CASSIN 1860: Av. PASSERIF. (Mesomyod.) Formicariid. (Grallarin.), serait syn. de Calobamon HEINE 1890.

Pituophis HOLBROOK 1842 : R.SQUAMAT. (Serpent.) Colubrid.

Pitylus CUVIER 1817: Av. PASSERIF. (Acromyod.) Fringillid. (Pitylin.).

Pitymys Mc MURTRIE 1831 : M.RODENT. (Myomorph.) Microtid.

Pityriasis LESSON 1839: Av. PASSERIF. (Acromyod.) Cracticid. ou Laniid.

Pityriopsis REICHENBACH 1850 : Av. PASSERIF. (Acromyod.) Laniid.

Pizonyx MILLER 1906: M.-CHIROPT. (Microchiropt.) Vespertilionid. (Vespertilionin.).

Placopharynx COPE 1870 : P.-CYPRINIF. (Cyprinoid.) Catostomid.
Placophorus MULSANT et VERREAUX 1874: Av. APODIF. Trochilid.

Placosoma FITZINGER 1847 : R.SQUAMAT. (Saur.) Tejid.

Placostomus RIDGWAY 1907 : syn. de Platyrinchus DESMAREST 1805 .

Placovaranus FEJEVARY 1927 : R.-SQUAMAT. (Saur.) Varanid.

Plagiodontia CUVIER 1836 : M. RODENT. (Caviomorph.) Echimyid. ou Myocastorid.

Plagiogenion FORBES 1891: P. PERCIF. (Percoid.) Serranid.

Plagiognathops BERG 1907: nom. nov. pro Plagiognathus DYBOWSKI 1872.

Plagiognathus DYBOWSKI 1872 : P.-CYPRINIF. (Cyprinoid.) Cyprinid.

Plagiopholis BOULENGER 1892 : R.-SQUAMAT. (Serpent.) Colubrid.

Plagiopsetta FRANZ 1910 : syn. de Samariscus GILBERT 1905.

Plagioscion GILL 1861 : P.-PERCIF. (Percoid.) Sciaenid.

Plagiospiza RIDGWAY 1898: Av. PASSERIF. (Acromyod.) Fringillid.

Plagiotremus GILL 1867 : P.-PERCIF. (Blennioid.) Blenniid.

Plagopterus COPE 1874: P.-CYPRINIF. (Cyprinoid.) Cyprinid.

Plagusia JAROCKI 1822 : P.-PLEURONECTIF. (Soleoid.) Cynoglossid. (serait syn. de Euporista GISTEL 1848).

Plagyodus STELLER 1814: syn. d'Alepisaurus LOWE 1833.

Planaltina : P.-CYPRINIF, (Characoid.) Characid.

Plancthanthias FOWLER 1935 : P.PERCIF. (Percoid.) Serranid.

Plancterus GARMAN : P.-PHALLOSTETHIF. Phallostethid.

Planesticus BONAPARTE 1854: Av. PASSERIF. (Acromyod.) Turdid. (Turdin.).

Planigale TROUGHTON 1928 : syn. de Phascogale TEMMIN CK 1824.

Planiprora WHITLEY 1931: P.- 
PERCIF. (Scorpaenoid.) Platycephalid.

Plargyrus RAFINESQUE 1820 : P.CYPRINIF. (Cyprinoid.) Cyprinid.

Platacanthomys BLYTH 1859 : M.RODENT. (Myomorph.) Platacanthomyid. (Platacanthomyin.).

Platacanthus FISHER de WALDHEIM 1849 : P.-CYPRINIF. (Cyprinoid.) Cobitid.

Platalea LINNE 1759 : Av. ARDEIF. Plataleid. (Platalein.).

Platalina THOMAS 1928: M.-CHIROPT. (Microchiropt.) Phyllostomatid. (Glossophagin.).

Platanista WAGLER 1830 nec CUVIER 1824: M.-CETAC. (Odontocet.) Platanistid. (Platanistin.).

Plataplochilus AHL 1928: P.-CYPRINODONTIF. Cyprinodontid.

Platax CUVIER 1817 : P.-PERCIF. (Percoid.) Ephippiid.

Plataxoides CASTELNAU 1855 : P.PERCIF. (Percoid.) Chaetodontid.

Plateibis BONAPARTE 1855: Av. ARDEIF. Plataleid. (Threskiornithin.).

Platemys WAGLER 1830: R.-TESTUDIN. (Thecophor.) Chelydid.

Platessa CUVIER 1817: P.-PLEURONECTIF. Pleuronectid.

Platichthys GIRARD 1856: P.PLEURONECTIF. (Pleuronectoid.) Pleuronectid.

Platinematichthys : vide Platyne matichthys BLEEKER 1863.

Platirostra LESUEUR 1818: syn. de Polyodon SCHNEIDER 1801.

Platistoma : vide Platystoma AGASSIZ 1829.

Platistus RAFINESQUE 1815: P.CYPRINIF. (Siluroid.) Bunocephalid.

Platophrys SWAINSON 1829 : syn. de Bothus BONAPARTE 1841.

Platopterus RAFINESQUE 1815 : P.-RAJIF. (Rajoid.) Rajid.

Platotichthys NICHOLS 1921: P.-
PLEURONECTIF. (Pleuronectoid.) Bothid.

Platuronides ROULE et BERTIN 1924: P.-CLUPEIF. (Nemichthyoid.) Serrivomerid.

Platurus LATREILLE 1801 : R.SQUAMAT. (Serpent.) Hydrophiid.

Platybelone FOWLER 1919 : P.BELONIF. (Belonoid.) Belonid.

Platyberyx ZUGMAYER 1911 : P.BER YCIF. Caristiid.

Platycara Mc CLELLAND 1839 : P.CYPRINIF. (Cyprinoid.) Cyprinid.

Platycephalops SMITH 1957 : P.PERCIF. (Gobioid.) Eleotrid.

Platycephalus BLOCH 1795 : P.PERCIF. (Scorpaenoid.) Platycephalid.

Platyceps BLYTH 1860 nec STEPHENS 1887 : R.-SQUAMAT. (Serpent.) Colubrid.

Platycercomys BRANDT 1844 : syn. de Pygeretmus GLOGER 1841.

Platycercus VIGORS 1825: Av. PSITTACIF. Psittacid.

Platyceros WAGNER 1844 : syn. de Dama GRAY 1825.

Platyceros ZIMMERMANN 1780 : syn. de Dama GRAY 1825.

Platycichla BAIRD 1864 : Av. PASSERIF. (Acromyod.) Turdid. (Myadestin.).

Platycranius KASCENKO 1901 : s. $/ g$. d'Alticola B L A N F O R D 1881.

Platydactylus GOLDFUSS 1820 : syn. de Tarentola GRAY 1825.

Platydoras BLEEKER 1862 : P.-CYPRINIF. (Siluroid.) Doradid.

Platygaster SWAINSON 1838: P.CLUPEIF. (Clupeoid.) Clupeid.

Platygeomys MERRIAM 1895 : M.RODENT. (Myomorph.) Geomyid.

Platyglossus BLEEKER 1862 : P.PERCIF. (Labroid.) Labrid.

Platvgobio GILL 1863: P.-CYPRINIF. (Cyprinoid.) Cyprinid.

Platyhyla BOULE NGER 1889 : Amph. ANOUR. Microhylid.

Platyinion AMARAL 1923 : R.SQUAMAT. (Serpent.) Boigid.

Ann. de Parisitologie, t. XXXVII, N ${ }^{\circ}$ 5-6. - 1962. 
Platyinius GILL 1863 : P.-PERCIF. (Percoid.) Lutjanid.

Platylepes SWAINSON 1839: P.PERCIF. (Percoid.) Lectariid.

Platylophus SWAINSON 1832: Av. PASSERIF. (Acromyod.) Prionopid.

Platymantis GUNTHER 1858 : Amph. ANOUR. Ranid.

Platymops THOMAS 1906 : syn. de Tadarida RAFINESQUE 1814.

Platynematichthys BLEEKER 1863: P.-CYPRINIF. (Siluroid.) Bagrid.

Platypanchax AHL 1928: P.-CYPRINODONTIF. Cyprinodontid.

Platypelis BOULEN GE R 1882 : Amph. ANOUR. Microhylid.

Platypharodon HERTZENSTEIN 1891: P.-CYPRINIF. (Cyprinoid.) Cyprinid.

Platypholis BOULENGER 1890 nec DUGES 1891 : R.-SQUAMAT. (Saur.) Geckonid.

Platyplectrurus GUNTHER 1868: R.-SQUAMAT. (Serpent.) Uropeltid.

Platypodon GILL 1862: P.-GALEIF. (Carcharhinoid.) Carcharhinid.

Platypodus de LACEPEDE 1804: P.-PERCIF. (Anabantoid.) Anabantid.

Platypoecilus GUNTHER 1866 : P.CYPRINODONTIF. Poecilid.

Platypogon STARKS 1913 : P.-CYPRINIF. (Siluroid.) Bagrid.

Platypsaris BONAPARTE 1854: Av. PASSERIF. (Mesomyod.) Cotingid.

Platyptera CUVIER 1829 : P.-PERCIF. (Gobioid.) Gobiid.

Platypus SHAW 1799 : syn. d'Ornithorhynchus BLUMENBACH 1800.

Platyrhina MULER et HENLE 1838: P.-RAJIF. (Rhinobatoid.) Rhinobatid.

Platyrinchus DESMAREST 1805: Av. PASSERIF. (Mesomyod.) Tyrannid. (Platyrhynchin.).

Platyrhinoides JORDAN 1881 : P.RAJIF. (Rhinobatoid.) Rhinobatid.
Platyrhynchus SWAINSON 1820 : emend. pro Platyrinchus DESMAREST 1805.

Platysaurus SMITH 1849: R.-SQUAMAT. (Saur.) Iguanid. ou Zonurid.

Platyschista OTTO 1835: syn. de Paradoxurus CUVIER 1821.

Platysilurus HASEMAN 1911: P.CYPRINIF. (Siluroid.) Bagrid.

Platysmurus REICHENBACH 1850 : Av. PASSERIF. (Acromyod.) Corvid.

Platysomatichthys BLEEKER 1862 : P. - PLEURONECTIF. (Pleuronectoid.) Bothid.

Platysqualus SWAINSON 1839 : P.$G A L E I F . \quad$ (Carcharhinoid.) Sphyrnid.

Platystacus BLOCH 1794: P.-CYPRINIF. (Siluroid.) Bunocephalid.

Platysteira JARDINE et SELBY 1829: Av. PASSERIF. (Acromyod.) Muscicapid.

Platysternum (GRAY 1831) AGASSIZ 1846 : R.-TESTUDIN. (Thecoph.) Platysternid.

Platystethus GUNTHER 1860 : P.PERCIF. (Stromateoid.) Stromateid.

Platystoma AGASSIZ 1829: P.-CYPRINIF. (Siluroid.) Bagrid.

Platystomatichthys BLEEKER 1862 : P.-CYPRINIF. (Siluroid.) Bagrid.

Platystomus FISHER de WALDHEIM 1803: syn. de Dugong de LACEPEDE 1799.

Platystylopterus REICHENBACH 1854 : syn. de Campylopterus SWAINSON 1827.

Platytaeniodus BOULENGER 1906 : P.-PERCIF. (Percoid.) Cichlid.

Platytriccus RIDGWAY 1905 : syn. de Platyrinchus DESMAREST 1805 .

Platytroctegen LLOYD 1909: P.CLUPEIF. (Alepocephaloid.) Alepocephalid.

Platytroctes GUNTHER 1878: P.CLUPEIF. (Alepocephaloid.) Alepocephalid.

Platvtropius HORA 1937: P.-CYPRINIF. (Siluroid.) Schilbeid. 
Platyuraspis FOWLER 1939 : P.PERCIF. (Percoid.) Carangid.

Plautus BRUNNICH 1772 : syn. de Alle LINK 1806.

Plecodus BOULENGER 1898: P.PERCIF. (Percoid.) Cichlid.

Plecoglossus SCHLEGEL 1850 : P.CLUPEIF. (Salmonoid.) Plecoglossid.

Plecostomus GRONOV 1763: P.-CYPRINIF. (Siluroid.) Loricariid.

Plecotus OKEN 1816: M.-CHIROPT. (Microchiropt.) Vespertilionid. (Vespertilionin.).

Plectodus : errat. pro Plecodus BOULENGER 1898.

Plectorhinchus de LA CEPEDE 1802: P.-PERCIF. (Percoid.) Lufjanid.

Plectorrhynchus BERTHOLD 1827: emend. pro Plectorhinchus de LACEPEDE 1802.

Plectranthias BLEEKER 1876 : P.PERCIF. (Percoid.) Serranid.

Plectrochilus MIRANDA-RIBEIRO 1917 : P.-CYPRINIF. (Siluroid.) Trichomycterid.

Plectrogenium GILBERT 1905 : P.PERCIF. (Scorpaenoid.) Scorpaenid.

Plectroglyphidodon FOWLER et BALL 1924:P.-

Plectrohyla BROCCHI 1877 : Amph. ANOUR. Hylid.

Plectromantis PETERS 1862: Amph. ANOUR. Leptodactylid.

Plectromus GILL 1883 : P.-STEPHANOBERYCIF. Melamphaeid.

Plectroperca PETERS 1864: P.PERCIF. (Percoid.) Serranid.

Plectrophallus F $O$ WL ER 1932: s./g. de Panamichthys HUBBS 1925.

Plectrophanes MEYER 1815: Av. PASSERIF. (Acromyod.) Fringillid. (Emberizin.).

Plectrophenax STEJNEGER 1882: Av. PASSERIF. (Acromyod.) Fringillid. (Emberizin.).

Plectropoma FLEMMING 1822 : emend. pro Plectropomus OKEN 1817.
Plectropomus OKEN 1817 : P.-PERCIF. (Percoid.) Serranid.

Plectropterus STEPHENS 1824: Av. ANATIF. Anatid. (Anserin.).

Plectrorhynchus WIEGMANN 1838: Av. PASSERIF. (Acromyod.) Zosteropid.

Plectrostethus MYERS 1935: P. PHALLOSTETHIF. Phallostethid.

Plectrostoma GISTEL 1848 : P.GALEIF. (Isuroid.) Isurid.

Plectrurus DUMERIL 1851： R.SQUAMAT. (Serpent.) Uropeltid.

Plectrypops GILL 1863 : P.-BERYCIF. Holocentrid.

Plegadis KAUP 1829: Av. ARDEIF. Plataleid. (Threskiornithin.).

Plerodus SCHULTZ 1897 : syn. de Suncus EHRENBERG 1833.

Plerotes A N D ER S E N 1910 : M.CHIROPT. (Megachiropt.) Pteropid. (Pteropin.).

Plesiodryas : Av. PASSERIF. (Acromyod.) Muscicapid.

Plesiogale POCOCK 1921 : syn. de Mustela LINNE 1758.

Plesionerca VAILLANT 1873 : P.PERCIF. (Percoid.) Etheostomid.

Plesiops OKEN 1817: P.-PERCIF. (Percoid.) Plesiopid.

Plesiositagra IREDALE et BANNERMAN 1921: Av. PASSERIF. (Acromyod.) Ploceid. (Plocein.).

Plestiodon DUMERIL et BIBRON 1839: R.-SQUAMAT. (Saur.) Scincid.

Plestiodontoides GIGLIOLI 1874: R.-SQUAMAT. (Saur.) Scincid.

Plethodectes COPE 1870 : P.-CYPRINIF. (Characoid.) Characid.

Plethodon TSCHUDI 1838 : Amph. UROD. Plethodontid.

Plethodontohyla BOULENGER 1882: Amph. ANOUR. Microhylid.

Pletholax COPE 1864: R.-SQUAMAT. (Saur.) Pygopodid.

Plethopsis BISHOP 1937: Amph. UROD. Plethodontid.

Pleuracromylodon GILL 1864 : P.- 
GALEIF. (Carcharhinoid.) Carcharhinid.

Pleurodeles MICHAHELLES 1830 : Amph. UROD. Salamandrid.

Pleurodema TSCHUDI 1838 : Amph. ANOUR. Leptodactylid.

Pleuroderma TSCHUDI 1838 : corrig. pro Pleurodema TSCHUDI 1838.

Pleurogadus JORDAN 1887: P.GADIF. Gadid.

Pleurogobius : P.-PERCIF. (Gobioid.) Eleotrid.

Pleurogrammus GILL 1861: P.PERCIF. (Scorpaenoid.) Hexagrammid.

Pleurolepis QUENSTEDT 1852 : P.PERCIF. (Percoid.) Etheostomid.

Pleuronectes MOREAU: syn. de Psettodes BENNETT 1831.

Pleuronectes LIN N E 1758: P.PLEURONECTIF. (Pleuronectoid.) Pleuronectid.

Pleuronichthys GIRARD 1856 : P.PLEURONECTIF. (Pleuronectoid.) Pleuronectid.

Pleuroperca FOWLER et BEAN 1930: s./g. de Plectropomus OKEN 1817.

Pleurophysus RIBEIRO 1918 : P.CYPRINIF. (Siluroid.) Trichomycterid.

Pleuroscopus BARNARD 1927 : P.PERCIF. (Trachinoid.) Uranoscopid.

Pleurosicya WEBER 1913 : P.-PERCIF. (Gobioid.) Gobiid.

Pleurothyris LOWE 1860 : P.-CLUPEIF. (Stomiatoid.) Sternoptychid.

Plica GRAY 1830 : R.-SQUAMAT. (Saur.) Iguanid.

Plicomugil SCHULTZ 1953 : P.-MUGILIF. Mugilid.

Plioambystoma ADAMS et MARTIN 1929 : Amph. UROD. Ambystomatid.

Pliocercus COPE 1860 : R.-SQUA$M A T$. (Serpent.) Colubrid.

Pliotrema REGAN 1906 : P.-SQUALIF. (Squalioid.) Pristiophorid.

Plitops FRASER-BRUNNER 1933 : P.-PERCIF. (Percoid.) Chaetodontid. ou Pomacanthid.
Ploceella OATES 1882: Av. PASSERIF. (Acromyod.) Ploceid. (Plocein.).

Plocepasser SMITH 1836 : Av. PASSERIF. (Acromyod.) Ploceid. (Euplectin.).

Ploceolus REICHENBACH 1863 : Av. PASSERIF. (Acromyod.) Ploceid.

Ploceus CUVIER 1817 : Av. PASSERIF. (Acromyod.) Ploceid. (Plocein.).

Plotosus de LACEPEDE 1803 : P. CYPRINIF. (Siluroid.) Plotosid.

Plumantennatus SCHULTZ 1957: s./g. d'Antennarius de LACEPEDE 1798.

Pluvialis BRISSON 1760 : Av. CHARADRIIF. Charadriid. (Charadriin.).

Pluvianellus (HOMBRON et JACQULNOT) GRAY 1846:Av. CHARADRIIF. Charadriid. (Charadriin.).

Pluvianus VIEILLOT 1816: Av. CHARADRIIF. Glareolid.

Pluviopsetta T A N A K A 1915 : P.PLEURONECTIF. (Pleuronectoid.) Pleuronectid.

Plyctolophus VIEILLOT 1816: Av. PSITTACIF. Psittacid.

Pneumabranchus Mc CLELLAND 1843 : P.-SYMBRANCHIF, Amphipnoid.

Pneumatophorus JORDAN et GILBERT 1883: P.-PERCIF. (Scombroid.) Scombrid.

Pnictes JORDAN 1919 : P.-PLEURONECTIF. (Soleoid.) Soleid.

Pnoepyga HODGSON 1844: Av. PASSERIF. (Acromyod.) Troglodytid.

Poblana de BUEN 1945 : P.-MUGILIF. Atherinid.

Pocockictis KRETZOI 1947: syn. de Mustela LINNE 1758.

Podager WAGLER 1832: Av. CAPRIMULGIF. Caprimulgid. (Caprimulgin.).

Podager GISTEL 1848 : syn. de Gerres QUOY et GAIMARD 1824.

Podanomalus WAITE 1898: syn. de Notomys LESSON 1842. 
Podarcis WAGLER $1830: s . / g$. de Lacerta LINNE 1758.

Podargus VIEILLOT 1818: Av. CAPRIMULGIF. Podargid.

Podasocys COUES 1866 : Av. CHARADRIIF. Charadriid. (Charadriin.).

Podica LESSON 1831: Av. RALLIF. Heliornithid.

Podiceps LATHAL 1787: Av. PYGOPOD. (Podicipitif.) Podicipitid.

Podicipes OKEN 1839 : syn. de Podiceps LATHAM 1787.

Podilimbus GRAY 1846: syn. de Podilymbus LESSON 1831.

Podilymbus LESSON 1831: Av. PYGOPOD. (Podicipitif.) Podicipitid.

Podoces FISHER de WALDHEIM 1821: Av. PASSERIF. (ACrOmyod.) Corvid.

Podocnemis WAGLER 1830: R.TESTUDIN. (Thecoph.) Pelomedusid.

Podogymnura MEARNS 1905 : M.INSECTIV. Erinaceid. (Echinosoricin.).

Podoleptus RAFINESQUE 1815 : P.-PERCIF. (Scorpaenoid.) Aploactid.

Podomys OSGOOD 1909 : s./g. de Peromyscus GLOGER 1841.

Podothecus GILL 1861: P.-PERCIF. (Cottoid.) Agonid.

Podoxymys ANTHONY 1929: M.RODENT. (Myomorph.) Cricetid. (Hesperomyin.).

Poecile KAUP 1829 : s./g. de Parus LINNE 1758.

Poecilia SCHNEIDER 1801 : P.-CYPRINODONTIF. Poeciliid.

Poecilichthys AGASSIZ 1854: P. PERCIF. (Percoid.) Etheostomid.

Poecilictis THOMAS et HINTON 1920 : M.-FIS SIP. Mustelid. (Mustelin.).

Poeciliopsis REGAN 1913 : P.-CYPRINODONTIF. Poeciliid.

Poecilistes HUBBS 1926 : P.-CYPRINODONTIF. Poeciliid.

Poecilobrycon EIGENMANN 1909 : P.-CYPRINIF. (Characoid.) Characid.
Poecilocephalus KAUP 1856: P.ANGUILLIF. (Congroid.)

Ophichthyid.

Poecilocharax EIGENMANN 1909 : P.-CYPRINIF. (Characoid.) Characid.

Poeciloconger GUNTHER 1872 : P.-ANGUILLIF. (Congroid.) Congrid.

Poecilodes STEINDACHNER 1863 : P.-CYPRINODONTIF, Poeciliid.

Poecilodryas GOULD 1865 : Av. PASSERIF. (Acromyod.) Muscicapid.

Poecilogale THOMAS 1895: M.FISSIP. Mustelid. (Mustelin.).

Poecilonetta STRICKLAND 1842: Av. ANATIF. Anatid. (Anatin.).

Poecilophis KAUP 1856: P.-ANGUILLIF. (Anguilloid.) Muraenid.

Poecilopsetta GUNTHER 1880 : P.PLEURONECTIF. (Pleuronectoid.) Pleuronectid.

Poecilopsis : errat. pro Poeciliopsis REGAN 1913.

Poecilornis KAUP 1844: Av. FALCONIF. Falconid. (Falconin.).

Poecilosomatops FOWLER 1906 : P.-CYPRINIF. (Characoid.) Characid.

Poecilothraupis CABANIS 1851: syn. d'Anisognathus REICHEN$B A C H 1850$.

Poecilothrissa REGAN 1917: P.CLUPEIF. (Clupeoid.) Clupeid.

Poecilotriccus B ERLEPSCH et TACZANOWSKI 1884: Av. PASSERIF. (Mesomyod.) Tyrannid.

Poecilurichthys GILL $1858: s . / g$. d'Astyanax BAIRD et GIRARD 1854.

Poëlagus ST-LEGER 1932: M.-LAGOMORPH. Leporid. (Leporin.).

Poemys THOMAS 1916 : syn. de Dendromus SMITH 1829.

Poeoptera BONAPARTE 1854: Av. PASSERIF. (Acromyod.) Sturnid. (Graculin.).

Poëphagus GRAY 1843: s./g. de BOS LINNE 1758.

Poephila GOULD 1842: Av. PAS- 
SERIF. (Acromyod.) Ploceid. (Estrildin.).

Poescopia GRAY 1864 : syn. de Megaptera GRAY 1946.

Pogonathus de LACEPEDE 1803 : P.-PERCIF. (Percoid.) Sciaenid.

Pogonathus BONAPARTE 1831: emend. pro Pogonias de LACEPEDE 1802.

Pogonatus BOSC 1818 : errat. pro Pogonathus de LACEPEDE 1803.

Pogoneleotris BLEEKER 1875 : P.PERCIF. (Gobioid.) Eleotrid.

Pogonia LEACH 1815: Av. PICIF. Capitonid.

Pogonias ILLIGER 1811: syn. de Pogonia LEACH 1815.

Pogonias de LACEPEDE 1802 : P.PERCIF. (Percoid.) Sciaenid.

Pogonichthys GIRARD 1854: $P$.CYPRINIF. (Cyprinoid.) Cyprinid.

Pogoniulus LAFRESNAYE 1842 : Av. PICIF. Capitonid.

Pogonocharax REGAN 1907: syn. de Nuria CUVIER et VALENCIENNES 1842.

Pogonocichla CABANIS 1847: Av. PASSERIF. (Acromyod.) Turdid. (Phoeniculin.).

Pogonoculius SMITH 1931 : P.PERCIF. (Gobioid.) Eleotrid.

Pogonogobius SMITH 1931: P. PERCIF. (Gobioid.) Gobiid.

Pogonolycus NORMAN 1937: P.PERCIF. (Gobioid.) Zoarcid.

Pogonomelomys RUMMLER 1936 : syn. de Melomys THOMAS 1922.

Pogonomys MILNE-E DW A R D S 1877 : M.-RODENT. (Myomorph.) Murid. (Murin.).

Pogonoperca GUNTHER 1859 : P.PERCIF. (Percoid.) Serranid.

Pogonophis MYERS 1941: P.-ANGUILLIF. (Congroid.) Ophichthyid.

Pogonopoma REGAN 1904 : P.-CYPRINIF. (Siluroid.) Loricariid.

Pogonopomoides : P.-CYPRINIF. (Siluroid.) Loricariid.

Pogonorhynchus HOEVEN 1833 : Av. PICIF. Capitonid,
Pogonornis BILLBERG 1828 : syn. de Pogonias ILLIGER 1811.

Pogonornis GRAY 1846: Av. PASSERIF. (Acromyod.) Meliphagid.

Pogonoscorpius REGAN 1908 : P.PERCIF. (Scorpaenoid.) Scorpaenid.

Pogonotriccus CABANIS et HEINE 1860: Av. PASSERIF. (Mesomyod.) Tyrannid. (Platyrhynchin.).

Pogonymus GOSLINE 1959 : P.PERCIF. (Callionymoid.) Callionymid.

Poiana GRAY 1865 : M.-FISSIP. Viverrid. (Viverrin.).

Poicephalus SWAINSON 1837 : Av. PSITTACIF. Psittacid.

Polemaëtus HEINE 1886 : syn. de Spizaëtus VIEILLOT 1816.

Polemistria CABANIS et HEINE 1860 : syn. de Lophornis LESSON 1829.

Polemius KAUP 1858 : P.-PERCIF (Scorpaenoid.) Scorpaenid.

Polemon JAN 1858: R.-SQUAMAT. (Serpent.) Boigid.

Poliailurus LONNBERG 1925 : syn. de Felis LINNE 1758.

Polidacnis CASSIN 1864: Av. PASSERIF. (Acromyod.) Coerebid.

Polioaëtus KAUP 1847: Av. FALCONIF. Falconid. (Pandionin.).

Poliocephalus SELBY 1840: Av. PYGOPOD. (Colymbif.) Colymbid. ou Podicipitid.

Poliocitellus HOWELL $1938: s . / g$. de Citellus OKEN 1816.

Poliohierax KAUP 1847: Av. FALCONIF. Falconid. (Falconin.).

Poliolais ALEXANDER 1903 : Av. PASSERIF. (Acromyod.) Sylviid.

Poliolimnas SHARPE 1893: Av. RALLIF. Rallid.

Poliolophus SHARPE 1877: Av. PASSERIF. (Acromyod.) Pycnonotid. ou Parid.

Poliomyias SHARPE 1879: Av. PASSERIF. (Acromyod.) Muscicapid.

Polionetta OATES 1899 : s./g. de Anas LINNE 1758, 
Poliopsar SHARPE 1888 : Av. PASSERIF. (Acromyod.) Sturnid. (Sturnin.).

Polioptila SCLATER 1855: Av. PASSERIF. (Acromyod.) Sylviid. (Polioptilin.).

Poliospiza BONAPARTE 1850 : Av. PASSERIF. (Acromyod.) Fringillid. (Fringillin.).

Polipicus CASSIN 1863 : Av. PICIF. Picid.

Polisornis Mc GREGOR 1927 : s./g. de Prionochilus STRICKLAND 1841.

Polistonemus GILL 1862 : P.-POLYNEMIF. Polynemid.

Polistotrema JORDAN et GILBERT 1881: s./g. de Bdellostoma MULLER 1835.

Prllachius NILSSON 1832 : P.-GADIF. Gadid.

Polutus : P.-PERCIF. (Percoid.) Lutjanid.

Polyacanthonotus BLEEKER 1874: P.-Notacanthif. Notacanthid.

Polyacanthus CUVIER 1829: syn. de Macropodus de LACEPEDE 1802.

Polyamblyodon NORMAN 1935 : P.-PERCIF. (Percoid.) Sparid.

Polyboroides SMITH 1830: Av. FALCONIF. Falconid. (Accipitrin.).

Polyborus VIEILLOT 1816: Av. FALCONIF. Falconid. (Falconin. ou Milvin.).

Polycaulus GUNTHER 1860: P.PERCIF. (Scorpaenoid.) Synancejid.

Polycentropsis BOULENGER 1901 : P.-PERCIF. (Percoid.) Nandid. ou Polycentrid.

Polycentrus MULLER et TROSCHEL 1848 : P.-PERCIF. (Percoid.) Nandid. ou Polycentrid.

Polychrus CUVIER 1817 : R.-SQUAMAT. (Saur.) Iguanid.

Polycirrhus BOCOURT 1868 : P.PERCIF. (Percoid.) Sciaenid.

Polyclemus BERG 1895 : nom. nov. pro Polycirrhus BOCOURT 1868.

Polydactylus de LACEPEDE 1803 : P.-POLYNEMIF. Polynemid.
Polydaedalus WALILER $1830: s . / g$. de Varanus RAFINESQUE 1815 .

Polyerata HEINE 1863: Av. APODIF. Trochilid.

Polyipnus GUNTHER 1887: P.CLUPEIF. (Stomiatoid.) Sternoptychid.

Polymeton Mc CULLOCH 1926: syn. de Yarrella GOODE et BEAN 1895.

Polymixia LOWE 1833 : P.-BERYCIF. Polymixinid.

Polynemus LINNE 1758 : P.-POLY NEMIF. Polynemid.

Polyodon SCHNEIDER 1801: P.ACIPENSERIF. Polyodontid.

Polyodontes LESSON 1832: R.SQUAMAT. (Serpent.) Colubrid.

Polyodontophis BOULENGER 1890 : R.-SQUAMAT. (Serpent.) Colubrid.

Polypedates ou Polypedotes TSCHUDI 1838 : Amph. ANOUR. Racophorid.

Polypera BURKE 1912: P.-PERCIF. (Cottoid.) Cyclopterid.

Polyplancta HEINE 1863 : Av. APODIF. Trochilid.

Polyplectron TEMMINCK 1807 : Av. PHASIANIF. Phasianid. (Phasianin.).

Polyprion OKEN 1817 : P.-PERCIF. (Percoid.) Serranid.

Polyprosopus COUCH 1862: P.GALEIF. (Isuroid.) Cetorhinid.

Polypterichthys BLEEKER 1853 : P.-AULOSTOMIF. Aulostomid.

Polynterus GEOFFROY-ST-HILAI$R E$ in de LACEPEDE 1803: P.-POLYPTERIF. Polypterid.

Polyspinus : P.-CLUPEIF. (Stomiatoid.) Sternoptychid.

Polysteganus KLUNZINGER 1870 : P.-PERCIF. (Percoid.) Sparid.

Polystictus REICHENBACH 1850 : Av. ANATIF. Anatid. (Anatin.).

Polystotrema : Cyclost. MYXINIF. Myxinid.

Polystrictus : Av. PASSERIF. (Mesomyod.) Tyrannid.

Polytelis WAGLER 1832 : Av. PSITTACIF. Psittacid.

Polytmus BRISSON 1760 : Av. APODIF. Trochilid. 
Polyuranodon KAUP 1856 : P.-ANGUILLIF. (Anguilloid.) Muraenid.

Pomacantha DUMERIL 1805: errat. pro Pomacanthus de LACEPEDE 1802.

Pomacanthis RAFINESQUE 1815 : nom. nov. pro Pomacanthus de LACEPEDE 1802.

Pomacanthodes GILL 1863 : P.PERCIF. (Percoid.) Pomacanthid. (ou Chaetodontid.).

Pomacanthops SMITH 1955: P.PERCIF. (Percoid.) Pomacanthid. (ou Chaetodontid.).

Pomacanthus de LACEPEDE 1802 : P.-PERCIF. (Percoid.) Pomacanthid. (ou Chaetodontid.).

Pomacentrus de LACEPEDE 1802 : P.-PERCIF. (Labroid.) Pomacentrid.

Pomadasyna FOWLER 1931 : s./g. de Pomadasys de LACEPEDE 1802.

Pomadasys de LACEPEDE 1802 : P.-PERCIF. (Percoid.) Lutjanid.

Pomanotis GUICHENOT 1847 : P.PERCIF. (Percoid.) Nandid.

Pomarea BONAPARTE 1854: Av. PASSERIF. (Acromyod.) Muscicapid.

Pomareopsis OUSTALET 1880 : Av. PASSERIF. (Acromyod.) Muscicapid.

Pomataprion GILL 1864 : P.-PERCIF. (Labroid.) Pomacentrid.

Pomatias SCHNEIDER 1801: P. CLUPEIF. (Clupeoid.) Clupeid.

Pomatoichthys : errat. pro Pomatomichthys GIGLIOLI 1880.

Pomatomichthys GIGLIOLI 1880 : P.-PERCIF. (Percoid.) Pomatomid.

Pomatomus de LACEPEDE 1802 : P.-PERCIF. (Percoid.) Pomatomid.

Pomatomus RISSO 1810 : syn. de Telescops BLEEKER 1876.

Pomatopsetta GILL 1864: P.PLEURONECTIF. (Pleuronectoid.) Pleuronectid.

Pomatorhinus HORSFIELD 1821: Av. PASSERIF. (Acromyod.) Timaliid. (Turdoidin.).
Pomatorhynchus HORS F IELD 1826: Av. PASSERIF. (Acromyod.) Laniid.

Pomatoschistus GILL 1864 : P.PERCIF. (Gobioid.) Gobiid.

Pomatostomus CABANIS 1851 : Av. PASSERIF. (Acromyod.) Timaliid. (Turdoidin.).

Pomatotis AGASSIZ 1846 : emend. pro Pomotis RAFINESQUE 1819.

Pomatotomus AGASSIZ 1846: emend. pro Pomatomus de LACEPEDE 1802.

Pomobolus : errat. pro Pomolobus RAFINESQUE 1820.

Pomodon BOULENGER 1894 : P.PERCIF. (Percoid.) Serranid.

Pomolobus RAFINESQUE 1820: P.-CLUPEIF. (Clupeoid.) Clupeid.

Pomotis RAFINESQUE 1819: P.PERCIF. (Percoid.) Centrarchid.

Pomoxis RAFINESQUE 1818: P. PERCIF. (Percoid.) Centrarchid.

Pomnilus MINDING 1832 : P.-PERCIF. (Stromateoid.) Stromateid.

Ponerodon ALCOCK 1890 : P.-PERCIF. (Chiasmodontoid.) Chiasmodontid.

Ponerovsar OBERHOLSER 1905 : Av. PASSERIF. (Acromyod.) Sturnid. (Graculin.).

Pongo de LACEPEDE 1799: M.PRIMAT. (Simioid.) Pongid.

Ponticola ILJIN 1927 : s./g. de Chaparrudo de BUEN 1931.

Pontinus POEY 1860 : P.-PERCIF. (Scorpaenoid.) Scorpaenid.

Pontodactylus : errat. pro Pantodactylus DUMERIL et BIBRON 1839.

Pontoporia GRAY 1846: syn. de Stenodelphis GERVAIS 1847.

Poocaetes : vide Pooecetes BAIRD 1858.

Pooecetes BAIRD 1858: Av. PASSERIF. (Acromyod.) Fringillid. (Emberizin.).

Poospiza CABANIS 1847 : Av. PASSERIF. (Acromyod.) Fringillid. (Fringillin.). 
Poospizopsis BERLEPSCH 1893 : Av. PASSERIF. (Acromyod.) Fringillid. (Fringillin.).

Pophiobagrus : P.-CYPRINIF. (Siluroid.) Bagrid.

Popelairia REICHENBACH 1854 : syn. de Lophornis LESSON 1829.

Poptella EIGENMANN 1909: P.CYPRINIF. (Characoid.) Characid.

Porcostoma : errat. pro Percostoma AGASSIZ 1845.

Porcula HODGSON 1847 : s./g. de Sus LINNE 1758.

Porcus GEOFFROY - ST - HILAIRE 1827 : P.-CYPRINIF. (Siluroid.) Bagrid.

Porichthys GIRARD 1854 : P.-PERCIF. (Batrachoidoid.) Batrachoidid.

Poroalticus F O W L E R 1931 : P.PERCIF. (Blennioid.) Blenniid.

Porochilus WEBER 1913: P.-CYPRINIF. (Siluroid.) Plotosid.

Poroclinus BEAN 1890: P.-PERCIF. (Blennioid.) Clinid.

Porocottus GILL 1859 : P.-PERCIF. (Cottoid.) Cottid.

Poroderma SMITH 1838 : P.-GALEIF. (Carcharhinoid.) Scyliorhinid.

Porogadus GOODE et BEAN 1885 : P.-PERCIF. (Ophidioid.) Brotulid.

Porogobius BLEEKER 1874 : P.PERCIF. (Gobioid.) Gobiid.

Porogrammus GILCHRIST et THOMPSON 1916: P.-PERCIF. (Blennioid.) Congrogadid.

Poromera BOULENGER 1887 : R.SQUAMAT. (Saur.) Lacertid.

Poromitra GOODE et BEAN 1883 : P. - STEPHANOBERYCIF. Melamphaeid.

Poromitrella ZUGMAYER 1911 : P.STEPHANOBERYCIF. Melamphaeid.

Poronotus GILL 1861 : P.-PERCIF. (Stromateoid.) Stromateid.

Poropuntius SMITH 1931: P.-CYPRINIF. (Cyprinoid.) Cyprinid.

Porotergus EIGENMANN 1912 : P.CYPRINIF. (Gymnotoid.) Sternarchid.
Porphyreocephalus REICHENOW 1880 : emend. pro Purpureicephalus BONAPARTE 1854.

Porphyrio BRISSON 1760: Av. RALLIF. Rallid.

Porphyriola SUNDEVALL 1873 : emend. pro Porphyrula BLYTH 1852 .

Porphyriops PUCHERAN 1845 : Av. RALLIF. Rallid.

Porphyriornis ALLEN 1892: Av. RALLLIF. Rallid.

Porphyrocephalus HEINE 1890 : syn. de Purpureicephalus $\mathrm{BO}$ NAPARTE 1854.

Pornhyrolaema BONAPARTE 1854: Av. PASSERIF. (Mesomyod.) Cotingid.

Porphyrospiza SCLATER 1873 : Av. PASSERIF. (Acromyod.) Fringillid.

Porphyrula BLYTH 1849: Av. RALLLIF. Rallid.

Portax SMITH 1827 : syn. de Boselaphus de BLAINVILLE 1816.

Porteridia FOWLER 1945 : P.-PERCIF. (Trachinoid.) Pinguipedid.

Porthmeus CUVIER et VALENCIENNES 1833: P. - PERCIF. (Percoid.) Carangid.

Porzana VIEILLOT 1816 : Av. RALLIF. Rallid.

Porzanula FROHAWK 1892: Av. RALLIF. Rallid.

Potamalosa OGILBY 1896 : P.-CLUPEIF. (Clupeoid.) Clupeid.

Potamochelidon HEINE 1890 : Av. PASSERIF. (Acromyod.) Hirundinid.

Potamochoerus GRAY 1852: M.PARAX. (Suif.) Suid. (Suin.).

Potamocottus GILL 1861: P.-PERCIF. (Cottoid.) Cottid.

Potamogale ALLEMAN 1865: M.INSECTIV. Tenrecid. (Potamogalin.).

Potamonhilus MULLER 1838 : syn. de Cynogale GRAY 1837.

Potamophis CANTOR 1836 : R.SQUAMAT. (Serpent.) Colubrid.

Potamorhina COPE 1878: P.-CYPRINIF. (Characoid.) Characid.

Potamorrhapis GUNTHER 1866 : P.-BELONIF. Belonid. 
Potamothrissa REGAN 1917: P. CLUPEIF. (Clupeoid.) Clupeid.

Potamotrygon GARMAN 1877: P. RAJIF. (Dasyatoid.) Dasyatid.

Potoroops MATSCHIE : M. -MARSUP. Macropodid. (Potoroin.).

Potorous DESMAREST 1804: M.MARSUP. Macropodid. (Potoroin.).

Potos GEOFFROY-ST-HILAIRE et CUVIER 1795 : M.-FISSIP. Procyonid. (Procyonin.).

Poutawa GRIFFIN 1936: P.-ANGUILLIF. (Congroid.) Congrid.

Praedo NELSON 1912: syn. d'Aphanotriccus RIDGWAY 1905.

Praeformosania FANG 1935: P.CYPRINIF. (Cyprinoid.) Homalopterıd.

Praesorex THOMAS 1913: M.-INSECTIV. Soricid. (Crocidurin.).

Pradjahipokia FOWLER 1934 : P.CYPRINIF. (Siluroid.) Bagrid.

Pranesella WHITLEY 1934: P.MUGILIF. Atherinid.

Pranesus WHITLEY 1930 : P.-MUGILIF. Atherinid.

Praomys THOMAS 1915: s./g. de Rattus FISCHER 1803.

Prasitis CABANIS et HEINE 1860 : Av. APODIF. Trochilid.

Praslinia BOULENGER 1909 : Amph. APOD. Caeciliid.

Pratincola DEGLAND 1849: syn. de Saxicola BECHSTEIN 1802.

Pratincola KOCH 1816: Av. PASSERIF. (Acromyod.) Muscicapid.

Premnas CUVIER 1817: P.-PERCIF. (Labroid.) Pomacentrid.

Premnocopus CABANIS 1847: Av. PASSERIF. (Mesomyod.) Dendrocolaptid.

Premnoplex CHERRIE 1891: Av. PASSERIF. (Mesomyod.) Dendrocolaptid.

Premnornis RIDGWAY 1909: Av. PASSERIF. (Mesomyod.) Dendrocolaptid.

Presbypithecus TROUESSART 1879: syn. de Semnopithecus CUVIER 1821.

Presbytis ESCHSCHOLTZ 1821: syn. de Semnopithecus CUVIER 1821.
Presbytiscus POCOCK 1924: s./g. de Rhinopithecus MILNE-EDWARDS 1872.

Prevagor: errat. pro Pervagor WHITLEY 1930.

Priacanthichthys DAY 1868: P.PERCIF. (Percoid.) Serranid.

Priacanthopsis FOWLER 1906 : P.PERCIF. (Percoid.) Pempherid.

Priacanthus OKEN 1817 : P.-PERCIF. (Percoid.) Serranid.

Prianella REGAN 1913 : P.-CYPRINODONTIF. Poeciliid.

Priapichthys REGAN 1913 : P.-CYPRINODONTIF. Poeciliid.

Prietella CARRANZA 1954 : P.-CYPRINIF. (Siluroid.) Bagrid.

Primospina EIGENMANN et BEESON $1893: s . / g$. de Sebastodes GILL 1861.

Prinia HORSFIELD 1821 : Av. PASSERIF. (Acromyod.) Sylviid.

Priniops ROBERTS 1922: Av. PASSERIF. (Acromyod.) Sylviid.

Priocella HOMBRON et JACQUINOT 1844: Av. PROCELLARIIF. Procellariid.

Priodon QUOY et GAIMARD 1824: P. - PERCIF. (Acanthuroid.) Acanthurid.

Priodonophis KAUP 1860 : P.-ANGUILLIF. (Anguilloid.) Muraenid.

Priodontes CUVIER 1822: M.EDENT. (Xenarthra.) Dasypodid. (Dasypodin.).

Priodontes LESSON 1842 : syn. de Prionodon HORSFIELD 1822.

Priodontichthys AGASSIZ 1846 : P.PERCIF. (Acanthuroid.) Acanthurid.

Priofinus HOMBRON et JACQUINOT 1844: Av. PROCELLARIIF. Procellariid.

Priolepis CUVIER et VALENCIENNES 1837: P.-PERCIF. (Gobioid.) Gobiid.

Prion de LACEPEDE 1799:Av. PROCELLARIIF. Procellariid.

Prionace CANTOR 1850 : nom. nov. pro Prionodon MULLER et HENLE 1841.

Prionailurus SEVERTZOV 1858: s./g. de Felis LINNE 1758. 
Prionirhynchus SCLATER 1858 : Av. CORACIADIF. Momotid.

Prionistius BEAN 1884: P.-PERCIF. (Cottoid.) Cottid.

Prioniturus WAGLER 1832: Av. PSITTACIF. Psittacid.

Prionobrama FOWLER 1913 : P.CYPRINIF. (Characoid.) Characid.

Prionobutis BLEEKER 1874: P.PERCIF. (Gobioid.) Eleotrid.

Prionochilus STRICKLAND 1841 : Av. PASSERIF. (Acromyod.) Dicaeid.

Prionodactylus O'SHAUGHNESSY 1881: syn. de Euspondylus TSCHUDI 1845.

Prionodes JENYNS 1840 : P.-PERCIF. (Percoid.) Serranid.

Prionodon HORSFIELD 1822 : M.FISSIP. Viverrid. (Viverrin.).

Prionodon MULLER et HENLE 1841: s./g. de Carcharhias RAFINESQUE 1810.

Prionodura de VIS 1883 : Av. PASSERIF. (Acromyod.) Paradiseid. (Paradisein.).

Prionolepis SMITH 1931: syn. de Monoceros de LACEPEDE 1798.

Prionolepis EGERTON 1850 : P.ZEIF. Zeid.

Prionomys DOLLMAN 1910: M.RODENT. (Myomorph.) Murid. (Dendromyin.).

Prionops VIEILLOT 1816: Av. PASSERIF. (Acromyod.) Prionopid.

Prionorhynchus : errat. pro Prionirhynchus SCLATER 1868.

Prionotelus REICHENBACH 1850 : Av. TROGONIF. Trogonid.

Prionotus de LACEPEDE 1802: P.-PERCIF. (Scorpaenoid.) Triglid.

Prionurus de LACEPEDE 1804: P. - PERCIF. (Acanthuroid.) Acanthurid.

Priopidichthys WHITLEY 1935 : P.-PERCIF. (Percoid.) Serranid.

Priopis KUHL et von HASSELT 1830 : syn. d'Ambassis CUVIER et VALENCIENNES 1828.
Pristella EIGENMANN 1909: P.CYPRINIF. (Characoid.) Characid.

Pristhocantharus : errat. pro Pristocantharus GILL 1863.

Pristhoplichthys : P.-PERCIF. (Scorpaenoid.) Platycephalid.

Pristiancistrus FOWLER 1945 : P.CYPRINIF. (Siluroid.) Loricariid.

Pristiapogon KLUNZINGER 1870 : P.-PERCIF, (Percoid.) Serranid.

Pristibatis de BLAINVILLE 1825 : P.-RAJIF. (Rhinobatoid.) Pristid.

Pristicharax : P.-CYPRINIF, (Characoid.) Characid.

Pristidactylus FITZINGER 1843 : R.-SQUAMAT. (Saur.) Iguanid.

Pristidoryrhamphus FOWLER 1944: P.SYNGNATHIF. Syngnathid.

Pristigaster CUVIER 1817 : P.CLUPEIF. (Clupeoid.) Clupeid.

Pristiodon DYBOWSKI 1877 : P.CYPRINIF. (Cyprinoid.) Cyprinid.

Pristiophorus MULLER et HENLE 1837 : P.-SQUALIF. (Squalioid.) Pristiophorid.

Pristiopsis LEICHARDT 1845 : P.RAJIF. (Rhinobatoid.) Pristid.

Pristipoma CUVIER 1829 : syn. de Pomadasys de LACEPEDE 1802.

Pristipomus SCHINZ 1822 : P.PERCIF. (Percoid.) Serranid.

Pristipomoides BLEEKER 1852: P.-PERCIF. (Percoid.) Lutjanid.

Pristis LINCK 1790: P.-R A JIF. (Rhinobatoid.) Pristid.

Pristiurus BONAPARTE 1831 : P.GALEIF. (Carcharhinoid.) Scyliorhinid.

Pristobrycon EIGENMANN 1915 : P.-CYPRINIF. (Characoid.) Characid.

Pristocantharus GILL 1863 : P.PERCIF. (Percoid.) Lutjanid.

Pristolepis JERDON 1849 : P.-PERCIF. (Percoid.) Nandid.

Pristorhamphus FINSCH 1876 : Av. 
PASSERIF. (Acromyod.) Drepanidid.

Pristotis RUPPELL 1838 : P.-PERCIF. (Labroid.) Pomacentrid.

Pristurus RUPPELL 1835 : R.SQUAMAT. (Saur.) Geckonid.

Proamblys GILL 1863 : P.-PERCIF. (Percoid.) Lutjanid.

Probarbus SAUVAGE 1880 : P.-CYPRINIF. (Cyprinoid.) Cyprinid.

Probassariscus MERRIAM 1911: M.-FISSIP. Procyonid. (Procyonin.).

Probolodus EIGENMANN 1911 : P.CYPRINIF. (Characoid.) Characid.

Proboscidiophis TSANG HOW FAN 1931 : R.-SQUAMAT. (Serpent.) Boigid.

Probosciger KUHL 1820 : Av. PSITTACIF. Psittacid.

Probreviceps PARKER 1931: Amph. ANOUR. Microhylid.

Procapra HODGSON 1846: M.-PARAX. Bovid. (Antilopin.).

Procapromys CHAPMAN 1901 : M.-RODENT. (Caviomorph.) Schimyid. (Capromyin.).

Procarduelis HODGSON 1844 : syn. de Carpodacus KAUP 1829.

Procatopus BOULENGER 1904 : P.CYPRINODONTIF, C y p rinodontid.

Procavia STORR 1780: M.-HYRACOID. Procaviid.

Procellaria LINNE 1758: Av. PROCELLARIIF. Procellariid.

Procellarus BONAPARTE 1854: Av. LARIF. Larid.

Procelsterna LAFRESNAYE 1842 : Av. LARIF. Larid. (Sternin.).

Proceriolotes MATHEWS 1925: Av. PASSERIF. (Acromyod.) Meliphagid.

Prochilodus AGASSIZ 1829 : P.-CYPRINIF. (Characoid.) Characid.

Prochilus ILLIGER 1811: syn. de Melursus MEYER 1793.

Prochilus WALBAUM 1792: P.PERCIF. (Labroid.) Pomacentrid.

Procinura COPE 1879: R.-SQUAMAT. (Serpent.) Boigid.
Procnias ILLIGER 1811: Av. PASSERIF. (Mesomyod.) Cotingid.

Procnopsis CABANIS 1844: Av. PASSERIF. (Acromyod.) Tanagrid.

Procolobus ROCHEBRUNE 1887 : syn. de Colobus ILLIGER 1811.

Procops POCOCK 1923: syn. de Muntiacus RAFINESQUE 1815.

Procottus GRATZIANOV 1902 : P.PERCIF. (Cottoid.) Cottid.

Proctoporus TSCHUDI 1845: R.SQUAMAT. (Saur.) Tejid.

Proctostegus NARDO 1827 : P.BRAMIF. Bramid.

Proctotretus DUMERIL et BIBRON 1837 : R.-SQUAMAT. (Saur.) Iguanid.

Procutropichthys : errat. pro Proeutropichthys HORA.

Procyllium : errat. pro Proscyllium HILGENDORF 1904.

Procyon STORR 1780 : M.-FISSIP. Procyonid. (Procyonin.).

Procypris LIN SHU YEN 1933 : P.CYPRINIF. (Cyprinoid.) Cyprinid.

Prodelphinus van BENEDEN et GERVAIS 1877 : syn. de Stenella GRAY 1866.

Prodelphinus GERVAIS 1880 : M.CETAC. (Odontocet.) Delphinid.

Proditor WHITLEY 1940 : P.-CLUPEIF. (Alepocephaloid.) Alepocephalid.

Prodontocharax PEARSON 1924: P. C Y PRINIF. (Characoid.) Characid.

Prodorcas POCOCK 1918 : syn. de Procapra HODGSON 1846.

Prodotiscus SUNDEVALL 1850 : Av. PICIF. Indicatorid.

Proechidna GERVAIS 1877: syn. de Zaglossus GILL 1877.

Proechimys ALLEN 1899: M.-RODENT. (Caviomorph.) Echimyid. (Echimyin.).

Proedromys THOMAS 1911: M.RODENT. (Myomorph.) Microtid.

Proeutropichthys HORA : P.-CYPRINIF. (Siluroid.) Schilbeid.

Proeutropius FOWLER $1936: \mathrm{s} . / \mathrm{g}$. 
de Eutropius MULLER et TROSCHEL 1849.

Profelis SEVERTZOV 1858: s./g. de Felis LINNE 1758.

Profundisudis HARRY $1953: \mathrm{s} . / \mathrm{g}$. de Notolepis DOLLO 1908.

Profundulus HUBBS 1925 : P.-CYPRINODONTIF. Cyprinodontid.

Progastromyzon HORA et JAYARAM 1952: P.-CYPRINIF. (Cyprinoid.) Homalopterid.

Prognathodus EGERTON 1872 : P.PERCIF. (Percoid.) Chaetodontid.

Progne BOIE 1826 : Av. PASSERIF. (Acromyod.) Hirundinid.

Prognichthys BREDER 1928: P.BELONIF. (Exoetoid.) Exocoetid.

Prognurus JORDAN et EVERMANN 1898: P.-PERCIF. (Cottoid.) Cyclopterid.

Prolabeo NORMAN 1932: P.-CYPRINIF. (Cyprinoid.) Cyprinid.

Prolabeops SCHULTZ 1941 : P.-CYPRINIF. (Cyprinoid.) Cyprinid.

Prolatilus GILL 1865 : P.-PERCIF. (Percoid.) Latilid.

Promacheon WEBER 1913 : P.CLUPEIF. (Alepisauroid.) Scopelarchid.

Promecocephalus TROSCHEL 1856 : P. - TETRAODONTIF. (Tetraodontoid.) Tetraodontid.

Promerluccius BOGHACHEV 1938 : P.-GADIF. Gadid.

Promerops BRISSON 1760: Av. PASSERIF. (Acromyod.) Meliphagid.

Prometheomys SATUNIN 1901: M.-RODENT. (Myomorph.) Microtid.

Promethichthys GILL 1893 : P.PERCIF. (Trichiuroid.) Gempylid.

Promicrops POEY 1868: P.-PERCIF. (Percoid.) Serranid.

Promicropterus GILL 1861: P.PERCIF. (Percoid.) Serranid.

Promops GERVAIS 1855: M.-CHIROPT. (Microchiropt.) Molossid.
Promyllantor ALCOCK 1890 : P.ANGUILLIF. (Congroid.) Congrid.

Pronolagus LYON 1904 : M.-LAGOMORPH. Leporid. (Palaeolagin.).

Pronotogrammus GILL 1864: P. PERCIF. (Percoid.) Serranid.

Proodus FOWLER 1944 : P.-

Proparoides BIANCHI 1902: Av. PASSERIF. (Acromyod.) Timaliid.

Proparus $H O D$ G S N 1845: Av. PASSERIF. (Acromyod.) Timaliid.

Propasser HODGSON 1844: syn. de Carpodacus KAUP 1829.

Prophagorus SMITH 1939 : P.-CYPRINIF, (Siluroid.) Clariid.

Prophetes VIGORS et HORSFIELD: Av. PASSERIF. (Acromyod.) Timaliid.

Propithecus BENNETT 1837: M.PRIMAT. (Lemur.) Indrid.

Propoma GUNTHER 1880 : P.-PERCIF. (Percoid.) Lutjanid.

Proportheus JAECKEL 1909 : P.CYPRINIF. (Characoid.) Characid.

Propseudecheneis HORA 1937 : P.CYPRINIF. (Siluroid.) Bagrid.

Propterygia OTTO 1820 : P.-RAJIF, (Rajoid.) Rajid.

Propus COPE 1874 : syn. de Ophiognomon COPE 1868.

Propyrrhula HODGSON 1844: Av. PASSERIF. (Acromyod.) Fringillid. (Fringillin.).

Propyrrhura RIBEIRO 1920: Av. PSITTACIF. Psittacid.

Proscelotes de WITTE et LAURENT 1942: R.-SQUAMAT. (Saur.) Scincid.

Proscyllium HILGENDORF 1904: P.-GALEIF. (Carcharhinoid.) Scyliorhinid.

Proscymnodon FOWLER 1934 : P.SQUALIF. (Squalioid.) Squalid.

Prosoarchus COHEN 1958 : s./g. de Glossanodon GUICHENOT 1867.

Prosobonia BONAPARTE 1850: Av. CHARADRIIF. Charadriid. (Scolopacin.). 
Prosonium JORDAN 1878 : P.-CLUPEIF. (Salmonoid.) Salmonid.

Prosoplismus WAITE 1903: P.PERCIF. (Percoid.) Lutjanid.

Prostheacanthus BLYTH 1860 : P.CYPRINIF. (Cyprinoid.) Cobitid.

Prosthemadera GRAY 1840: Av. PASSERIF. (Acromyod.) Meliphagid.

Prostherapis COPE 1868: Amph. ANOUR. Ranid.

Prosymma GRAY 1849 : R.-SQUAMAT. (Serpent.) Colubrid.

Proteles GEOFFROY-ST - HILAIRE 1824: M.-FISSIP. Hyaennid. (Protelin.).

Protemnodon $O W E N$ 1873 : M.MARSUP. Macropodid. (Macropodin.).

Proteocephalus : P.-PERCIF. (Scorpaenoid.) Platycephalid.

Proteracanthus GUNTHER 1859: P.-PERCIF. (Percoid.) Sparid.

Proterorhinus SMITT 1899: P.PERCIF. (Gobioid.) Gobiid.

Proteus LAURENTI 1768: Amph. UROD. Proteid.

Protistius COPE 1874: P.-MUGILIF. Atherinid.

Protocampus GUNTHER 1879 : P.SYNGNATHIF. Syngnathid.

Protocephalus : P.-CLUPEIF. (Mormyroid.) Mormyrid.

Protockus ROBERTS 1931 : syn. de Lophoceros HEMPRICH et EHRENBERG 1833.

Protomelus HOGG 1841: syn. de Protopterus OWEN 1837.

Protomyzon HORA 1932: P.-CYPRINIF. (Cyprinoid.) Homalopterid.

Protonotaria BAIRD 1858: Av. PASSERIF. (Acromyod.) Parulid.

Protopipa NOBLE 1925: Amph. ANOUR. Pipadid.

Protoporus COPE 1872 : P.-CYPRINIF. (Cyprinoid.) Cyprinid.

Protopsetta SCHMIDT 1904: P.PLEURONECTIF. (Pleuronectoid.) Pleuronectid.

Protopterus OWEN 1837: P.-CERATODIF. Lepidosirenid.
Prototroctes GUNTHER 1864 : P.CLUPEIF. (Galaxioid.) Aplochitonid.

Protoxerus FORSYTH-MAJOR 1893: M.-RODENT. (Sciuromorph.) Sciurid. (Sciurin.).

Protozygaena WHITLEY 1940 : syn. de Physodon MULLER et HENLE 1839.

Proturacopsis von BOETTICHER $1947:$ s./g. de Touraco de LACEPEDE 1799.

Protucarus BATES 1923: Av. CUCULLIF. Musophagid.

Prox OGILBY 1837 : syn. de Muntiacus RAFINESQUE 1815.

Prunella VIEILLOT 1816: Av. PASSERIF. (Acromyod.) Prunellid.

Prymnomiodon C O P E 1861 : R.SQUAMAT. (Serpent.) Colubrid.

Prymnothonus RICHARDSON 1845: P.-CLUPEIF. (Myctophoid.) Sudid.

Prymnothonoides WHITLEY et PHILLIPPS 1939: P. -CLUPEIF. (Myctophoid.) Sudid.

Przewalskia KOZLOVA 1946: Av. COLUMBIF. (Pteroclidif.) Pteroclidid.

Przewalskium FLEROV $1930: s . / g$. de Cervus LINNE 1758.

Psalidodon EIGENMANN 1911 : P.CYPRINIF. (Characoid.) Characid.

Psalidoprocne CABANIS 1851: Av. PASSERIF. (Acromyod.) Hirundinid.

Psalidoprymna CABANIS et HEINE 1860: Av. APODIF. Trochilid.

Psalidostoma KNER 1865: P.-CYPRINIF. (Characoid.) Characid.

Psaltria TEMMINCK 1836: Av. PASSERIF. (Acromyod.) Parid.

Psaltriparus BONAPARTE 1850 : Av. PASSERIF. (Acromyod.) Parid.

Psammichthys REGAN 1908: P.PERCIF. (Gobioid.) Gobiid.

Psammoaëtus ROBERTS 1924: Av. FALCONIF. Falconid. (Aquilin.).

Psammobates FITZINGER 1835: 
R. - TESTUDIN. (Thecophor.) Testudinid.

Psammobatis GUNTHER 1870 : P.-RAJIF. (Rajioid.) Rajiid. (serait syn. de Malacorhina GAR$M A N$ 1877).

Psammocrex OUSTALET 1886: Av. RALLIF. Rallid.

Psammodiscus GUNTHER 1862: P. - PLEURONECTIF. (Pleuronectoid.) Pleuronectid.

Psammodromus FITZINGER 1826: R.-SQUAMAT. (Saur.) Lacertid.

Psammodynastes GUNTHER 1858 : R.-SQUAMAT. (Serpent.) Boigid.

Psammoenas BLYTH 1845: Av. COLUMBIF. Columbid.

Psammogobius SMITH 1935: P.PERCIF. (Gobioid.) Gobiid.

Psammomys CRETZCHMAR 1828: M. -RODENT. (Myomorph.) Gerbillid.

Psammoperca RICHARDSON 1848: P.-PERCIF. (Percoid.) Serranid.

Psammôphilus FITZINGER 1843 : R.-SQUAMAT. (Saur.) Agamid.

Psammophis FITZINGER 1826 : R.SQUAMAT. (Serpent.) Boigid.

Psammophylax FITZINGER 1843 : R.-SQUAMAT. (Serpent.) Boigid.

Psammosaurus FITZINGER 1826 : syn. de Varanus MERREM 1820.

Psaris CUVIER 1817: Av. PASSERIF. (Mesomyod.) Cotingid.

Psarisomus ou Psarisoma SWAINSON 1837: Av. PASSERIF. (Desmodactyl.) Eurylaimid.

Psarocolius WAGLER 1827: Av. PASSERIF. (Acromyod.) Icterid.

Psaropholus JARDINE et SELBY 1838: s./g. d'Oriolus LINNE 1766.

Psectrogaster EIGENMANN 1889 : P.-CY P RINIF. (Characoid.) Characid.

Psednos BARNARD 1927 : P.-PERCIF. (Cottoid.) Cyclopterid.

Pselaphias JORDAN et SEALE 1906: P.-PERCIF. (Gobioid.) Gobiid.
Pselaphon GRAY 1870 : syn. de Pteropus BRISSON 1762.

Pselliophorus RIDGWAY 1898 : Av. PASSERIF. (Acromyod.) Fringillid.

Psellogrammus EIGENMANN 1909: P.-CY PR I N IF. (Characoid.) Characid.

Psenes CUVIER et VALENCIENNES 1833: P.-PERCIF. (Stromateoid.) Stromateid.

Psephotellus MATHEWS 1913: Av. PSITTACIF. Psittacid.

Psephotus GOULD 1845 : Av. PSITTACIF. Psittacid.

Psephurus GUNTHER 1873 : P.ACIPENSERIF. Polyodontid.

Psetta SWAINSON 1839 : P.-PLEURONECTIF. (Pleuronectoid.) Pleuronectid.

Psettias JORDAN 1906 : P.-PERCIF. (Percoid.) Monodactylid.

Psettichthys GIRARD 1856 : P.PLEURONECTIF. (Pleuronectoid.) Pleuronectid.

Psettina HUBBS 1915 : P.-PLEURONECTIF. (Pleuronectoid.) Bothid.

Psettodes BENNETT 1831: P.PLEURONECTIF. (Psettodoid.) Psettodid.

Psettus CUVIER 1817 : syn. de Monodactylus de LACEPEDE 1802.

Psettylis ALCOCK 1890 : P.-PLEURONECTIF. (Pleuronectoid.) Bothid.

Pseudablabes BOULENGER 1896 : R.-SQUAMAT. (Serpent.) Boigid.

Pseudacontias HEWITT 1929 : R.SQUAMAT (Saur.) Scincid.

Pseudacris FIT ZINGER 1843: Amph. ANOUR. Hylid.

Pseudaëton BUTURLIN 1910: Av.

Pseudaesopia CHABANAUD 1934 : s./g. de Zebrias JORDAN et SNYDER 1900.

Pseudaëtus BONAPARTE 1854: syn. de Hieraëtus GRAY 1845.

Pseudagelaeus RIDGWAY 1901: Av. PASSERIF. (Acromyod.) Icterid.

Pseudageniosus BLEEKER 1863 : 
P.-CYPRINIF. (Siluroid.) Doradid.

Pseudagkistrodon van DENBURG 1910:R.-SQUAMAT. (Serpent.) Crotalid.

Pseudalaemon PHILLIPS 1898: Av. PASSERIF. (Acromyod.) Alaudid.

Pseudalaudula BIANCHI 1906 : Av. PASSERIF. (Acromyod.) Alaudid.

Pseudalopex BURMEISTER 1856 : s./g. de Dusicyon SMITH 1839.

Pseudalphestes BOULENGER 1894: P.-PERCIF. (Percoid.) Serranid.

Pseudambassis BLEEKER 1876: P.-PERCIF. (Percoid.) Serranid.

Pseudamia BLEEKER 1865: P. PERCIF. (Percoid.) Serranid.

Pseudamiatus WHITLEY 1954 : P.PERCIF. (Percoid.) Serranid.

Pseudamiops SMITH 1954 : P.-PERCIF. (Percoid.) Serranid.

Pseudammomanes BIANCHI 1905 : Av. PASSERIF. (Acromyod.) Alaudid.

Pseudanampses BLEEKER : P.PERCIF. (Labroid.) Labrid.

Pseudancistrus BLEEKER 1862: P.-CYPRINIF. (Siluroid.) Loricariid.

Pseudantechinus TATE 1947: M. MARSUP. Dasyurid. (Dasyurin.).

Pseudanthias BLEEKER 1873 : P.PERCIF. (Percoid.) Serranid.

Pseudanthropos REICHENBACH 1862 : syn. de Pan OKEN 1816.

Pseudaphya ILJIN 1930 : P.-PERCIF. (Gobioid.) Gobiid.

Pseudaphritis CASTELNAU 1872 : P.-PERCIF. (Trachinoid.) Trachinid.

Pseudapistocalamus LONNBERG 1900: syn. de Ultrocalamus STERNFELD 1913.

Pseudapocryptes BLEEKER 1874 : P.-PERCIF. (Gobioid.) Gobiid.

Pesudariodes BLEEKER 1862 : P.CYPRINIF. (Siluroid.) Bagrid.

Pseudarius BLEEKER 1862: P.CYPRINIF. (Siluroid.) Bagrid.
Pseudarthroleptis DECKERT 1938: Amph. ANOUR. Ranid.

Pseudaspis FITZINGER 1843 : R.SQUAMAT (Serpent.) Colubrid.

Pseudaspius DYBOWSKI 1869 : P.CYPRINIF. (Cyprinoid.) Cyprinid.

Pseudastrapia ROTSCHILD 1907: Av. PASSERIF. (Acromyod.) Paradiseid. (Paradisein.).

Pseudattila ZIM MER 1936: Av. PASSERIF. (Mesomyod.) Cotingid.

Pseudauchenipterus B LE E K E R 1862 : P.-CYPRINIF. (Siluroid.) Doradid.

Pseudaxis GRAY 1872: syn. de Cervus LINNE 1758.

Pseudecheneis BLYTH 1860 : P.CYPRINIF. (Siluroid.) Bagrid.

Pseudechidna BLEEKER 1863 : P.ANGUILLIF. (Anguilloid.) $\mathrm{Mu}$ raenid.

Pseudechis W A GLER 1830: R.SQUAMAT. (Serpent.) Elapid.

Pseudelaps DUMERIL 1853: R.SQUAMAT. (Serpent.) Elapid.

Pseudemydura SIEBENROCK 1901: R. - TESTUDIN. (Thecoph.) Chelyid.

Pseudemys GRAY 1855 : R.-TESTUDIN. (Thecoph.) Emydid.

Pseudengystoma de WITTE 1930 : syn. de Asterophrys TSCHUDI 1838.

Pseudeos PETERS 1935 : Av. PSITTACIF. Psittacid.

Pseudepapterus STEINDACHNER 1917 : P.-CYPRINIF. (Siluroid.) Doradid.

Pseuderemias BOETTEGER 1883 : R.-SQUAMAT. (Saur.) Lacertid.

Pseuderythrinus : P.-CYPRINIF. (Characoid.) Characid.

Pseudeutropichthys KOLLER 1926: syn. de Cranoglanis PETERS 1881.

Pseudeutropius BLEEKER 1863 : P.-CYPRINIF. (Siluroid.) Schilbeid.

Pseudhymenochirus CHABANAUD 1920: Amph. ANOUR. Pipadid.

Pseudibis HODGSON 1844: Av. ARDEIF. Plataleid. (Threskiornithin.). 
Pseudis WAGLER 1830: Amph. ANOUR. Hylid.

Pseudoacontias du BOCAGE 1889 : vide Pseudacontias HEWITT 1929.

Pseudoalcippe BANNERMAN 1923 : Av. PASSERIF. (Acromyod.) Timaliid.

Pseudoambassis CASTELNAU 1878: syn. de Blandowskiella IREDALE et WHITLEY 1932.

Pseudoammomanes : vide Pseudammomanes BIANCHI 1905.

Pseudobagrichthys BLEEKER 1862 : P.-CYPRINIF. (Siluroid.) Bagrid.

Pseudobagrus BLEEKER 1858 : P.CYPRINIF. (Siluroid.) Bagrid.

Pseudobarbus BIELZ 1853 : P.-CYPRINIF. (Cyprinoid.) Cyprinid.

Pseudobatrachus CASTELNAU 1875: P. - PERCIF. (Batrachoid.) Batrachoidid.

Pseudobias SHARPE 1870: Av. PASSERIF. (Acromyod.) Muscicapid.

Pseudoblennius T EMMINCK et SCHLEGEL 1850: P.-PERCIF. (Blennioid.) Blenniid.

Pseudoboa SCHNEIDER 1801 : R.SQUAMAT. (Serpent.) Boigid.

Pseudoboodon PERACCA 1897 : R.-SQUAMAT. (Serpent.) Colubrid.

Pseudobrama BLEEKER 1870 : P.CYPRINIF. (Cyprinoid.) Cyprinid.

Pseudobranchus GRAY 1825: Amph. UROD. Sirenid.

Pseudobucco des MURS 1853: Av. PICIF. Capitonid.

Pseudobufo TSCHUDI 1838: Amph. ANOUR. Bufonid.

Pseudobulweria MATHEWS 1936 : Av. PROCELLARIIF. Procellariid.

Pseudobythites MEEK et HILDEBRAND 1928 : syn. de Petrotyx HELLER et SNODGRASS 1903.

Pseudocadia LINDHOLM 1931 : R.TESTUDIN. (Thecoph.) Emydid.

Pseudocallophysus $B L E E K E R$ 1863 : P.-CYPRINIF. (Siluroid.) Bagrid.
Pseudocalyptomena ROTSCHILD 1909 : Av. PASSERIF. (Desmodactyl.) Eurylaimid.

Pseudocaranx BLEEKER : P.-PERCIF. (Percoid.) Carangid.

Pseudocepola KAMOHARA 1935 : P.-PERCIF. (Percoid.) Cepolid.

Pseudoceramodactylus HAAS 1957: R.-SQUAMAT. (Saur.) Geckonid.

Pseudocerastes BOULENGER 1896: R.-SQUAMAT. (Serpent.) Viperid.

Pseudocervus HODGSON 1841 : syn. de Cervus LINNE 1758.

Pseudocetopsis BLEEKER 1862 : P.-CYPRINIF. (Siluroid.) Silurid.

Pseudochaenichthys NORMAN 1937 : P.-PERCIF. (Trachinoid.) Trachinid.

Pseudochalceus KNER et STEINDACHNER 1863: P.-C Y PRINIF. (Characoid.) Characid.

Pseudocheilinus BLEEKER 1862 : P.-PERCIF. (Labroid.) Labrid.

Pseudocheirodon MEEK et HILDEBRAND 1916: P. - CYPRINIF. (Characoid.) Characid.

Pseudocheirus OGILBY 1838: M.MARSUP. Phalangerid. (Phascolarctin.).

Pseudochelidon HARTLAUB 1861: Av. PASSERIF. (Acromyod.) Vangid.

Pseudochirops MATSCHIE 1915: M. - MARSUP. Phalangerid. (Phascolarctin.).

Pseudochlamydera MATHEWS 1926: Av. PASSERIF. (Acromyod.) Paradiseid. (Ptilonorhynchin.).

Pseudochloris SHARPE 1888: Av. PASSERIF. (Acromyod.) Fringillid. (Fringillin.).

Pseudochromichthys SCHMIDT 1931: P.-PERCIF. (Percoid.) Serranid.

Pseudochromis RUPPELL 1835: P.-PERCIF. (Percoid.) Serranid.

Pseudociccaba KELSO 1932: s./g. de Ciccaba WAGLER 1832.

Pseudocirrhites NOWBRAY 1927: P.-PERCIF. (Percoid.) Cirrhitid. 
Pseudocitharichthys WEBER 1913: P. - PLEURONECTIF. (Pleuronectoid.) Bothid.

Pseudoclinus SCHLEGEL 1850: errat. pro Pseudoblennius TEMMINCK et SCHLEGEL 1850.

Pseudoclelia RENDHAL et VESTERGREN 1941： R.-SQUAMAT. (Serpent.) Boigid.

Pseudocolaptes REICHENBACH 1853: Av. PASSERIF. (Mesomyod.) Furnariid.

Pseudocolopteryx LILLE 1905: Av. PASSERIF. (Mesomyod.) Tyrannid. (Platyrhinchin.).

Pseudoconomys RHOADS 1896 : syn. de Mus LINNE 1758.

Pseudocordylus SMITH 1838: R.SQUAMAT. (Saur.) Zonurid.

Pseudocoris BLEEKER 1862 : P.PERCIF. (Labroid.) Labrid.

Pseudocorynopoma PERUGIA 1891: P.-CYPRINIF. (Characoid.) Characid.

Pseudocossyphus SHARPE 1883 : Av. PASSERIF. (Acromyod.) Timaliid.

Pseudocrenilabrus FOWLER 1934: P.-PERCIF. (Percoid.) Serranid.

Pseudoculter BLEEKER 1860 : P.CYPRINIF. (Cyprinoid.) Cyprinid.

Pseudocurimata : P.-CYPRINIF. (Characoid.) Characid.

Pseudocyclophis BOETTE GER 1888 : R.-SQUAMAT. (Serpent.) Colubrid.

Pseudocyttus GILCHRIST 1907: P.-ZEIF. Zeid.

Pseudodacnis SCLATER 1887: Av. PASSERIF. (Acromyod.) Tanagrid.

Pseudodax BLEEKER 1861: P.PERCIF. (Labroid.) Odacid.

Pseudodipsas PETERS 1860:R.SQUAMAT. (Serpent.) Boigid.

Pseudodistira KINGHORN 1926 : R. -SQUAMAT. (Serpent.) Hydrophiid.

Pseudodon KESSLER 1874 : P.-CYPRINIF. (Cyprinoid.) Cobitid.

Pseudodoras BLEEKER 1858: P.CYPRINIF. (Siluroid.) Doradid.
Pseudoechidna : vide Pseudechidna $B L E E K E R 1863$.

Pseudoeryx FITZINGER 1826 : R.SQUAMAT. (Serpent.) Colubrid.

Pseudoeurycea TAYLOR 1944: Amph. UROD. Plethodontid.

Pseudoficimia BOCOURT 1883 : R.SQUAMAT. (Serpent.) Colubrid.

Pseudofringilla H U M E 1873 : Av. PASSERIF. (Acromyod.) Fringillid.

Pseudogallirex von BOETTICHER $1947: s . / g$. de Touraco de LACEPEDE 1799.

Pseudogastromyzon NICHOLS 1925: P. -CYPRINIF. (Cyprinoid.) Homalopterid.

Pseudogecko: vide Pseudogekko TAYLOR 1922.

Pseudogekko TAYLOR 1922: R.SQUAMAT. (Saur.) Geckonid.

Pseudogenetta DEKEYSER 1949 : M.-FISSIP. Viverrid. (Viverrin.).

Pseudogeranus SHARPE 1883: Av. FALCONIF, Falconid.

Pseudogerygone SHARPE 1878: syn. de Gerygone GOULD 1841.

Pseudoglottis STEJNEGER 1884: Av. CHARADRIIF. Charadriid. (Scolopacin.).

Pseudogobio BLEEKER 1860 : P.CYPRINIF. (Cyprinoid.) Cyprinid.

Pseudogobiodon BLEEKER 1874: P.-PERCIF. (Gobioid.) Gobiid.

Pseudogobiops BERG 1914 : P.-CYPRINIF. (Cyprinoid.) Cyprinid.

Pseudogobiopsis KOUMANS 1935 : P.-PERCIF. (Gobioid.) Gobiid.

Pseudogobius (?) : P.-PERCIF. (Gobioid.) Gobiid.

Pseudogonatodes RUTHVEN 1915: R.-SQUAMAT. (Saur.) Geckonid.

Pseudogramma BLEEKER 1875 : P.-PERCIF. (Percoid.) Serranid.

Pseudogyps SHARPE 1873: Av. FALCONIF. Falconid. (Aegypiin.). 
Pseudogyrinocheilus FANG 1933 : P.-CYPRINIF. (Cyprinoid.) Cyprinid.

Pseudohelotes GUIMARAES 1882: P.-PERCIF. (Percoid.) Serranid.

Pseudohemiculter NICHOLS et POPE 1927: syn. de Hainania KOLLER 1926.

Pseudohemiodon BLEEKER 1862 : P.-CYPRINIF. (Siluroid.) Loricartid.

Pseudohemisus MOCQUARD 1895 : Amph. ANOUR. Microhylid.

Pseudohomaloptera SILAS 1953: P.-CYPRINIF. (Cyprinoid.) Homalopterid.

Pseudohydromys RUMMLER 1934 : M.-RODENT. (Myomorph.) Murid. (Hydromyin.).

Pseudohyla ANDERSSON 1945: Amph. ANOUR. Hylid.

Pseudohymenochirus : vide Pseudhymenochirus CHABANAUD 1920.

Pseudois HODGSON 1846 : M.-PARAX. Bovid. (Caprin.).

Pseudojulis BLEEKER 1862: P.PERCIF. (Labroid.) Labrid.

Pseudojuloides FOWLER 1949 : P.PERCIF. (Labroid.) Labrid.

Pseudojulops FOWLER 1941 : P.PERCIF. (Labroid.) Labrid.

Pseudolabrus BLEEKER 1862 : P.PERCIF. (Labroid.) Labrid.

Pseudolais VAILLANT 1902: P.CYPRINIF. (Siluroid.) Schilbeid.

Pseudolalage BL Y T H 1861: Av. PASSERIF. (Acromyod.) Campephagid.

Pseudolalage MATHEWS 1928: syn. de Neolalage MATHEWS 1928.

Pseudolaubuca BLEEKER 1863 : P.-CYPRINIF. (Cyprinoid.) Cyprinid.

Pseudoleistes SCLATER 1862: Av. PASSERIF. (Acromyod.) Icterid. (Agelaein.).

Pseudoleptodeira TAYLOR 1938: R.-SQUAMAT. (Serpent.) Boigid.

Pseudoloricaria BLEEKER 1862 : P.-CYPRINIF. (Siluroid.) Loricariid.
Pseudomnila OATES 1894: syn. d'Alcippe BLYTH 1844.

Pseudomoho MATHEWS 1925: Av. PASSERIF. (Acromyod.) Meliphagid.

Pseudomonacanthus $B L E E K E R$ 1866: P.-PERCIF. (Balistoid.) Balistid. ou Monacanthid.

Pseudomopus HEWITT 1931 : syn. de Homopus DUMERIL et BIBRON 1835.

Pseudomoringua BLEEKER 1865 : P. - ANGUILLIF. (Anguilloid.) Moringuid.

Pseudomugil KNER 1866 : P.-MUGILIF. Atherinid.

Pseudomulloides RIBEIRO 1915: P.-PERCIF. (Percoid.) Mullid.

Pseudomuraena JOHNSON 1862 : P. - ANGUILLIF. (Anguilloid.) Muraenid.

Pseudomycterus OGILBY 1908 : P.PERCIF. (Percoid.) Sciaenid.

Pseudomyiobius SALVADORI et FESTA 1899: Av. PASSERIF. (Mesomyod.) Tyrannid.

Pseudomyrophis WADE 1946 : P.ANGUILLIF. (Congroid.) Echelid.

Pseudomys GRAY 1832: M.-RODENT. (Myomorph.) Murid. (Murin.).

Pseudonaja GUNTHER 1858: syn. de Demansia GRAY 1842.

Pseudonestor ROTSCHILD 1893: Av. PASSERIF. (Acromyod.) Drepanidid.

Pseudonigrita REICHENOW 1903 : Av. PASSERIF. (Acromyod.) Ploceid.

Pseudopaludicola MIRANRA-RIBEIRO 1926: Amph. ANOUR. Leptodactylid.

Pseudopangasius BLEEKER 1862 : P.-CYPRINIF. (Siluroid.) Schilbeid.

Pseudopareas BOULENGER 1896 : R.SQUAMAT. (Serpent.) Colubrid.

Pseudopentaceros BLEEKER 1876: P.-PERCIF. (Percoid.) Lutjanid.

Pseudopercis RIBEIRO 1903 : P.PERCIF. (Percoid.) Cichlid.

Pseudoperilampus BLEEKER 
1863 : P.-CYPRINIF. (Cyprinoid.) Cyprinid.

Pseudophallus HERALD 1940 : P.SYNGNATHIF. Syngnathid.

Pseudophichthys ROULE 1915 : P.ANGUILLIF. (Congroid.) Congrid.

Pseudophilautus LAURENT 1943 : Amph. ANOUR. Racophorid.

Pseudophiops JERDON 1870 : R.SQUAMAT. (Saur.) Lacertid.

Pseudophoxinus BLEEKER 1860: P.-CYPRINIF. (Cyprinoid.) Cyprinid.

Pseudophryne FITZINGER 1843 : Amph. ANOUR. Leptodactylid.

Pseudophycis GUNTHER 1862 : syn. de Physiculus KAUP 1858.

Pseudopimelodus BLEEKER 1858 : P.-CYPRINIF. (Siluroid.) Bagrid.

Pseudoplatichthys HIKITA 1934: P. -PLEURONECTIF. (Pleuronectoid.) Pleuronectid.

Pseudoplatystoma BLEEKER 1862: P.-CYPRINIF. (Siluroid.) Bagrid.

Pseudoplectrurus BOULENGER $1890: R .-S Q U A M A T$. (Serpent.) Uropeltid.

Pseudoplesiops BOULENGER 1899: P.-PERCIF. (Percoid.) Serranid. ou Plesiopid.

Pseudopleuronectes BLEE KER 1862 : P.-PLEURONECTIF. (Pleuronectoid.) Pleuronectid.

Pseudopodoces LARUDNY et LOVDON 1902: Av. PASSERIF. (Acromyod.) Corvid.

Pseudopoëtus von BOETTICHER 1947: s./g. de Touraco de LACEPEDE 1799.

Pseudopomacentrus $B L E E K E R$ 1868: P.-PERCIF. (Labroid.) Pomacentrid.

Pseudopriacanthus BLEEKER 1869 : P.-PERCIF. (Percoid.) Serranid.

Pseudoprion COUES 1866: Av. PROCELLARIIF. Procellariid.

Pseudopristipoma SAUVAGE 1880: P.-PERCIF. (Percoid.) Lutjanid.

Pseudoprosymna LINDHOLM
1902 : R.-SQUAMAT. (Serpent.) Colubrid.

Pseudopsectrogaster : P. - CYPRINIF. (Characoid.). Characid.

Pseudopterus WALBAUM 1792 : P. - PERCIF. (Scorpaenoid.) Scorpaenid.

Pseudoptynx KAUP 1848: Av. STRIGIF. Strigid. (Bubonin.).

Pseudopungtungia MORI 1935 : P.CYPRINIF. (Cyprinoid.) Cyprinid.

Pseudopus MERREM 1820 : syn. de Ophisaurus DAUDIN 1803.

Pseudoraja BIGELOW et SCHROEDER 1954 : P.-RAJIF. (Rajoid.) Rajid.

Pseudorasbora BLEEKER 1860 : P.-CYPRINIF. (Cyprinoid.) Cyprinid.

Pseudorca REINHARDT 1863 : M.CETAC. (Odontocet.) Delphinid.

Pseudorhabdion BOULENGER 1885: R.-SQUAMAT. (Serpent.) Colubrid.

Pseudorhadinea BOETTEGER 1898: syn. de Pararhadinea BOETTEGER 1898.

Pseudorhectes SHARPE 1877 : Av. PASSERIF. (Acromyod.) Prionopid.

Pseudorhinelepis : vide Pseudorinelepis BLEEKER 1862.

Pseudorhombus BLEEKER 1862 : P. - PLEURONECTIF. (Pleuronectoid.) Bothid.

Pseudorinelepis BLEEKER 1862 : P.-CYPRINIF. (Siluroid.) Loricaritd.

Pseudoryzomys HERSHKOWITZ 1959: M.-RODENT. (Myomorph.) Cricetid. (Cricetin.).

Pseudosalamandra TSCHUDI 1838: Amph. UROD. Salamandrid.

Pseudoscapirhynchus NIKOLSKI 1900 : P.-ACIPENSERIF. Acipenserid.

Pseudoscarus BLEEKER 1861 : syn. de Callyodon GRONOV 1763.

Pseudosciaena BLEEKER 1863 : P. -PERCIF. (Percoid.) Sciaenid. 
Pseudoscopelus LUTKEN 1892 : P.PERCIF. (Chiasmodontoid.) Chiasmodontid.

Pseudoscops KAUP 1848: Av. STRIGIF. Strigid. (Strigin.).

Pseudoscymnus HERRE 1935 : P.SQUALIF. (Squalioid.) Scymnorhinid.

Pseudosebastes SAUVAGE 1878 : P.-PERCIF. (Scorpaenoid.) Scorpaenid.

Pseudoserranus KLUNZINGER 1870: P.-PERCIF. (Percoid.) Serranid.

Pseudosilurus BLEEKER 1858 : P.CYPRINIF. (Siluroid.) Silurid.

Pseudospermestes DUBOIS 1905: syn. de Spermestes SWAINSON 1837.

Pseudosphromenus BLEEKER 1879 : s./g. de Macropodus de LACEPEDE 1802.

Pseudospingus BERLEPSCH et STOLZMANN 1896: Av. PASSERIF. (Acromyod.) Fringillid.

Pseudospiza SHARPE 1876: Av. PASSERIF. (Acromyod.) Fringillid.

Pseudostegophilus EIGENMANN et EINGENMANN 1889 : P.CY. PRINIF. (Siluroid.) Trichomycterid.

Pseudostomias FOWLER 1934 : P.CLUPEIF. (Stomiatoid.) Stomiatid.

Pseudosynanceja DAY 1875 : P.PERCIF. (Scorpaenoid.) Synancejid.

Pseudosyngnathus KNER et STEINDACHNER 1863 : P.SYNGNATHIF. Syngnathid.

Pseudosynodontis BLEEKER 1862: P.-CYPRINIF. (Siluroid.) Loricariid.

Pseudotadorna KURODA 1917 : Av. ANATIF. Anatid. (Anatin.).

Pseudotantalus RIDGWAY 1883 : Av. ARDEIF. Ciconid.

Pseudotestudo LOVERIDGE et WILLIAMS $1957 \mathrm{~s}$ s./g. de Testudo LINNE 1758.

Pseudothecadactylus BRONGERSMA 1936: R.-SQUAMAT. (Saur.) Geckonid.

Pseudothyrina RIBEIRO 1915 : P.MUGILIF. Atherinid.
Pseudotocinclus NICHOLS 1919 : P.-CYPRINIF. (Siluroid.) Loricariid.

Pseudotolithus BLEEKER 1863 : P.-PERCIF. (Percoid.) Sciaenid.

Pseudotomodon KOSLOWSKY 1897 : R.-SQUAMAT. (Serpent.) Boigid.

Pseudototanus HUME 1878: Av. CHARADRIIF. Charadriid. (Scolopacin.).

Pseudotrachinus BLEEKER 1861: P.-PERCIF. (Trachinoid.) Trachinid.

Pseudotriacanthus FRASER-BRUNNER 1940:P.-PERCIF. (Balistoid.) Triacanthid.

Pseudotriacis GUNTHER 1870 : emend. pro Pseudotriakis CAPELLO 1868.

Pseudotriakis CAPELLO 1868 : P.GALEIF. (Carcharhinoid.) Scyliorhinid.

Pseudotriccus TACZANOWSKI 1885: Av. PASSERIF. (Mesomyod.) Tyrannid.

Pseudotrionyx DOLLO 1886 : R.TESTUDIN. (Thecoph.) Trionychid.

Pseudotriton TS CHUDI 1838 : Amph. UROD. Plethodontid.

Pseudotropheus REGAN 1921 : P.PERCIF. (Percoid.) Cichlid.

Pseudotrypauchen HARDENBERG 1931: P.-PERCIF. (Gobioid.) Gobiid.

Pseudotylosurus FERNANDEZYPEZ 1948 : P.-BELONIF. (Belonoid.) Belonid.

Pseudovis GILL 1872: errat. pro Pseudois HODGSON 1846.

Pseudoxenicus FINSCH 1900: Av. PASSERIF. (Acromyod.) Sylviid.

Pseudoxenodon BOULENGER $1890: R .-S Q U A M A T$. (Serpent.) Colubrid.

Pseudoxenomystax BREDER 1927 : syn. de Congrina JORDAN et HUBBS 1925.

Pseudoxenopus BARBOUR et LOVERIDGE 1927 : syn. de Rana LINNE 1758.

Pseudoxiphophorus BLEEKER 
1860 : P. -CYPRINODONTIF. Poeciliid.

Pseudoxyrhopus GUNTHER 1881: R.-SQUAMAT. (Serpent.) Colubrid.

Pseudozosterops FINSCH 1901 : Av. PASSERIF. (Acromyod.) Zosteropid.

Pseudupeneus BLEEKER 1863: P.-PERCIF. (Percoid.) Mullid.

Pseuduromacer WERNER 1924 : R.-SQUAMAT (Serpent.) Colubrid. ou Boigid.

Psilocephalus SWAINSON 1839 : P.-PERCIF. (Balistoid.) Balistid.

Psilocranium MACLEAY 1884 : P.PERCIF. (Percoid.) Cirrhitid.

Psilodactylus GRAY 1864: R.SQUAMAT. (Saur.) Geckonid.

Psilodraco NORMAN 1937 : P.-PERCIF. (Trachinoid.) Nototheniid.

Psiloides FISHER de WALDHEIM 1813 : P.-PERCIF. (Gobioid.) Eleotrid.

Psilomycter HARTERT 1899: Av.

Psilopentapodus FOWLER 1933 : s./g. de Pentapodus QUOY et GAIMARD 1824.

Psilopogon MULLER 1835 : Av. PICIF. Capitonid.

Psilopornis RIDGWAY 1914: Av. PICIF. Galbulid.

Psilopsiagon RIDGWAY 1912: Av. PSITTACIF. Psittacid.

Psilorhamphus SCLATER 1955: Av. PASSERIF. (Mesomyod.) Petroptochid. (Rhinocryptin.).

Psilorhinus RUPPELL 1837: Av. PASSERIF. (Acromyod.) Corvid.

Psilorhynchus Mc CLELLAND 1839: P.-CYPRINIF. (Cyprinoid.) Cyprinid.

Psilorhynchus SAUVAGE et DA$B R Y$ : syn. d'Hemimyzon REGAN 1911.

Psilosomus SWAINSON 1839 : P.PERCIF. (Gobioid.) Gobiid.

Psilus FISHER de WALDHEIM 1813 : P. - PERCIF (Gobioid.) Eleotrid.

Psitodos MATHEWS 1929 : syn. de Todopsis BONAPARTE 1854.
Psittacara VIGORS 1825 : Av. PSITTACIF. Psittacid.

Psittacella SCHLEGEL 1871: Av. PSITTACIF. Psittacid.

Psittacirostra TEMMINCK 1820 : Av. PASSERIF. (Acromyod.) Drepanidid. ou Dicaeid.

Psittacula CUVIER 1800 : Av. PSITTACIF. Psittacid.

Psittacus LINNE 1758: Av. PSITTACIF. Psittacid.

Psitteuteles BONAPARTE 1854: Av. PSITTACIF. Psittacid.

Psittinus BLYTH 1842: Av. PSITTACIF. Psittacid.

Psittiparus HELLMAYR 1903 : syn. de Paradoxornis GOULD 1836.

Psittospiza BONAPARTE 1850: Av. PASSERIF. (Acromyod.) Tanagrid.

Psittrichas LESSON 1831: Av. PSITTACIF. Psittacid.

Psomocolax PETERS 1929: Av. PASSERIF. (Acromyod.) Icterid. (Cacicin.).

Psomophilus ROBERTS 1922: Av. PASSERIF. (Acromyod.) Motacillid.

Psophia LINNE 1758 : Av. RALLIF. Psophiid.

Psophocichla CABANIS 1860: Av. PASSERIF. (Acromyod.) Timaliid.

Psophodes VIGORS et HORSFIELD 1826: Av. PASSERIF. (Acromyod.) Timaliid.

Psychichthys FOWLER 1907 : P.-HOLOCEPHAL. Chimaerid.

Psychrolutes GUNTHER 1861 : P.PERCIF. (Cottoid.) Cottid.

Psychromaster JORDAN et EVERMANN 1896 : P.-PERCIF. (Percoid.) Etheostomid.

Psychropoecilia MYERS 1935 : P.CYPRINODONTIF. Poeciliid.

Ptarmus : P.-PERCIF. (Scorpaenoid.) Scorpaenid.

Ptax JORDAN et EVERMANN 1927: P.-PERCIF. (Trachinoid.) Gempylid.

Ptenochirus PETERS 1861: M.CHIROPT. (Megachiropt.) Pteropid. (Pteropin.).

Ptenotus OGLLBY : syn. de Parexocoetus BLEEKER 1866. 
Ptenopus GRAY 1866: R.-SQUAMAT. (Saur.) Geckonid.

Pteraclidus RAFINESQUE 1815: P.-BRAMIF. Bramid.

Pteraclis GRONOV 1763 : P.-BRAMIF. Bramid. ou Petraclid.

Petracryptis : P.-CYPRINIF. (Siluroid.) Silurid.

Pteraëtus KAUP 1847: Av. FALCONIF. Falconid. (Aquilin.).

Pteragogus PETERS 1855 : P.-PERCIF. (Labroid.) Labrid.

Pteralopex THOMAS 1888 : syn. de Pteropus BRISSON 1762.

Pteranthias WEBER 1913 : P.-PERCIF. (Percoid.) Serranid.

Pterapogon KOUMANS 1933: P.PERCIF. (Percoid.) Serranid.

Pterapon GRAY 1835 : emend. ou errat. pro Therapon CUVIER et VALENCIENNES 1829.

Pterarchis : P.-BRAMIF. Bramid.

Pteraspis KNER 1847 : P.-

Ptereleotris GILL 1864: P.-PERCIF. (Gobioid.) Eleotrid.

Pterengraulis GUNTHER 1868 : P.CLUPEIF. (Clupeoid.) Engraulid.

Ptererythrius STRICKLAND 1841 : emend. pro Pteruthius SWAINSON 1832.

Pterichthyodes BLEEKER 1959 : P.-PERCIF. (Scorpaenoid.) Scorpaenid.

Pterichthys SWAINSON 1839 : P.PERCIF. (Scorpaenoid.) Scorpaenid.

Pteridium SCOPOLI 1777 : syn. de Grammonus GOODE et BEAN 1895.

Pteridophora MEYER 1894: Av. PASSERIF. (Acromyod.) Paradiseid. (Paradisein.).

Pternistis WA GLER 1832: Av. PHASIANIF. Phasianid. (Phasianin.).

Pternohyla BOULENGER 1882: Amph. ANOUR. Hylid.

Pternopterus PETERS 1867: syn. de Myotis KAUP 1829.

Pterobalaena ESCHRICHT 1849 : syn. de Balaenoptera de LACEPEDE 1804.

Pterobrycon EIGENMANN 1913 :
P.-CYPRINIF. (Characoid.) Characid.

Pterocaesio BLEEKER 1875 : P.PERCIF. (Percoid.) Lutjanid.

Pterocapoeta GUNTHER 1902 : P.CYPRINIF. (Cyprinoid.) Cyprinid.

Pterocephala SWAINSON 1839: P.-RAJIF. (Dasyatoid.) Mobulid.

Pterochelidon : errat. pro Petrochelidon CABANIS 1851.

Pterocles TEMMINCK 1815: Av. COLUMBIF. (Pteroclidif.) Pteroclidid.

Pteroclurus BONAPARTE 1856: Av. COLUMBIF, (Pteroclidif.) Pteroclidid.

Pterocnemia GRAY 1871: Av. STRUTHIONIF. Rheid.

Pterocorys STEJNEGER 1884: Av. PASSERIF. (Acromyod.) Alaudid.

Pteroculiops FOWLER 1938 : P.PERCIF. (Gobioid.) Eleotrid.

Pterocyclostoma FOWLER 1941: P.-PERCIF. (Labroid.) Pomacentrid.

Pterocyon PETERS 1861: syn. de Eidolon RAFINESQUE 1815.

Pterodiscus EIGENMANN 1909 : P.CYPRINIF. (Characoid.) Characid.

Pterodoras BLEEKER 1862 : P.CYPRINIF. (Siluroid.) Doradid.

Pterodroma BONAPARTE 1955 : syn. de Aestrelata BONAPARTE 1856.

Pteroglanis FOWLER 1934: syn. de Pteropsoglanis FOWLER 1934.

Pteroglanis EIGENMANN et PEARSON 1924 : P.-CYPRINIF. (Siluroid.) Schilbeid.

Pteroglossus ILLIGER 1811: Av. PICIF. Rhamphastid.

Pterogobius GILL 1864: P.-PERClF. (Gobioid.) Gobiid.

Pterogymnus SMITH 1938 : P.PERCIF. (Percoid.) Sparid.

Pterohemiodus FOWLER 1940 : P.-CYPRINIF. (Characoid.) Characid.

Pteroidichthys BLEEKER 1856 ; 
P.-PERCIF. (Scorpaenoid.)

Scorpaenid.

Pteroidonus GUNTHER 1887 : P.PERCIF. (Ophidioid.) Brotulid.

Pterois OKEN 1817: P.-PERCIF. (Scorpaenoid.) Scorpaenid.

Pterolamia BREUNING 1942 : P.GALEIF. (Carcharhinoid.) Carcharhinid.

Pterolamia SPRINGER 1950 : syn. de Petrolamiops SPRINGER 1951.

Pterolamiops SPRINGER 1951 : P.GALEIF. (Carcharhinoid.) Carcharhinid.

Pterolebias GARMAN 1895 : P.-CYPRINODONTIF. Cyprinodontid.

Pteroleptus : errat. pro Chiroleptus SWAINSON 1839 (Scorpaenid.).

Pterolestes SUNDEVALL 1874 : Av. FALCONIF. Falconid.

Pteromugil : P.-MUGILIF. Mugilid.

Pteromylaeus GARMAN 1913: P.RAJIF. (Dasyatoid.) Myliobatid.

Pteromys CUVIER 1800: M.-RODENT, (Sciuromorph.) Sciurid. (Petauristin.).

Petromyscus THOMAS 1908: M.RODENT. (Sciuromorph.) Sciurid. (Petauristin.).

Pteronemus Van der HOE VEN 1833 : P.-PERCIF. (Percoid.) Cheilodactylid.

Pteronetta SALVADORI 1895: Av. ANATIF. Anatid. (Anatin.).

Pteronotropis FOWLER $1935: s . / g$. de Erogala JORDAN 1878.

Pteronotus SWAINSON 1839 : P.BELONIF. (Exocoetoid.) Exocoetid.

Petronotus GRAY 1838: M.-CHIROPT. (Microchiropt.) Phyllostomatid. (Phyllostomatin.).

Pteronura GRAY 1837 : M.-FISSIP. Mustelid. (Lutrin.).

Pteropangasius FOWLER 1938 : P.-CYPRINIF. (Siluroid.) Schilbeid.

Pteropelor F O WLER 1938 : P.PERCIF. (Scorpaenoid.) Scorpaenid.

Pterophanes GOULD 1849: Av. APODIF. Trochilid.
Pterophryne GILL 1864: P.-LOPHIIF. (Antennarioid.) Antennariid.

Pterophyllum HECKEL 1840 : P.PERCIF. (Percoid.) Cichlid.

Pteroplatea MULLER et HENLE 1837 : syn. de Gymnura KUHL 1823.

Pteroplatytrygon FOWLER 1910 : P.-RAJIF. (Dasyatoid.) Dasyatid.

Pteropodocys GOULD 1846: Av. PASSERIF. (Acromyod.) Campephagid.

Pteropodus EIGENMANN et BEESON 1893: s./g. de Sebastichthys GILL 1863.

Pteropsarion GUNTHER 1868 : P.CYPRINIF. (Cyprinoid.) Cyprinid.

Pteropsaron JORDAN et SNYDER 1902: P.-PERCIF. (Trachinoid.) Trachinid.

Pteropsoglanis FOWLER 1934 : P.CYPRINIF. (Siluroid.) Bagrid.

Pteropterus SWAINSON 1839 : P.PERCIF. (Scorpaenoid.) Scorpaenid.

Pteroptochus KITTLITZ 1830 : Av. PASSERIF. (Mesomyod.) Pteroptochid.

Pteropus BRISSON 1762 : M.-CHIROPT. (Megachiropt.) Pteropid. (Pteropin.).

Pterorhinus SWINHOE 1868: Av. PASSERIF. (Acromyod.) Timaliid. (Turdoidin.).

Petrorhombus FOWLER 1906 : P.PERCIF. (Stromateoid.) Stromateid.

Pteroscirtes : errat. pro Petroscirtes RUPPELL 1830.

Pterosmaris FOWLER 1925: P.PERCIF. (Percoid.) Dipterygonotid.

Pterothius : errat. pro Ptererythrius STRICKLAND 1841.

Pterothrissus HILGENDORF 1877 : P.-CLUPEIF. (Elopoid.) Pterothrissid.

Pterotolithus FOWLER $1933: s . / g$. d'Otolithus CUVIER 1829.

Pterozygus PYLAIE de FOUGERES 1835: P.-CYPRINIF. (Cyprinoid.) Cobitid. 
Pterurus RAFINESQUE 1810 : P.ANGUILLIF. (Anguilloid.) Moringuid.

Pteruthius SWAINSON 1832: Av. PASSERIF. (Acromyod.) Timaliid. ou Vangid.

Pterycombus FRIES 1838 : P.-BRAMIF. Bramid.

Pterygiocottus BEAN et WEED 1919: P.-PERCIF. (Cottoid.) Cottid.

Pterygistes KAUP 1829: M.-CHIROPT. (Microchiropt.) Vespertilionid. (Vespertilionin.).

Pterygocolobus ROCHEBRUNE 1886: syn. de Colobus ILLIGER 1811.

Pterygoplichthys GILL 1858 : P.CYPRINIF. (Siluroid.) Loricariid.

Pterygotrigla WAITE 1899 : P.PERCIF. (Scorpaenoid.) Triglid.

Pterythius : vide Pteruthius SWAINSON 1832.

Ptilinopus SWAINSON 1825: Av. COLUMBIF. Columbid. (Treronin.).

Ptilocercus GRAY 1848 : M.-INSECTIV. Tupaid. (Ptilocercin.).

Ptilochloris SWAINSON 1837: Av. PASSERIF. (Mesomyod.) Piprid.

Ptilocolpa BONAPARTE 1854: Av. COLUMBIF. Columbid. (Columbin.).

Ptilogonys SWAINSON 1837: Av. PASSERIF. (Acromyod.) Bombycillid.

Ptilolaemus GRANT 1892: Av. CORACIADIF. Bucerotid.

Ptilonorhynchus KUHL 1820 : Av. PASSERIF. (Acromyod.) Paradiseid. (Ptilonorhynchin.).

Ptilopachus SWAINSON 1837: Av. PHASIANIF. Phasianid. (Phasianin:).

Ptiloprora de VIS 1894: Av. PASSERIF. (Acromyod.) Meliphagid.

Ptilopus STRICKLAND 1841 : errat. pro Ptilinopus SWAINSON 1825.

Ptilopyga SHARPE 1883: syn. de Malacocincla BLYTH 1845.
Ptilorhis AGASSIZ 1845: Av. PASSERIF. (Acromyod.) Paradiseid. (Paradisein.).

Ptilorhynchus TEMMINCK 1826 : syn. de Ptilonorhynchus KUHL 1820.

Ptilorrhoa PETERS 1940: Av. PASSERIF. (Acromyod.) Timaliid.

Ptiloscelys BONAPARTE 1856: Av. CHARADRIIF. Charadriid. (Charadriin.).

Ptilosclera BONAPARTE 1857: Av. PSITTACIF. Psittacid.

Ptilostomus SWAINSON 1837: Av. PASSERIF. (Acromyod.) Corvid.

Ptilotis SWAINSON 1837 : Av. PASSERIF. (Acromyod.) Meliphagid.

Ptiloxena CHAPMAN 1892 : Av. PASSERIF. (Acromyod.) Icterid.

Ptistes GOULD 1865: Av. PSITTACIF. Psittacid.

Ptochoptera ELLIOTT 1874: Av. APODIF. Trochilid.

Ptyas FITZINGER 1843 : R.-SQUAMAT. (Serpent.) Colubrid.

Ptychadena BOULENGER 1917 : Amph. ANOUR. Hylid.

Ptychemys AGASSIZ 1857 : syn. de Pseudemys GRAY 1855.

Ptychidio MYERS 1930 : P.-CYPRINIF. (Cyprinoid.) Cyprinid.

Ptychobarbus STEINDACHNER 1866 : P.-CYPRINIF, (Cyprinoid.) Cyprinid.

Ptychocheilus AGASSIZ 1855: P.CYPRINIF. (Cyprinoid.) Cyprinid.

Ptychochromis STEINDACHNER 1880 : P.-PERCIF. (Percoid.) Cichlid.

Ptychoglossus BOULENGER 1890 : R.-SQUAMAT. (Saur.) Tejid.

Ptychoglossus RUIBAL: syn. de Proctoporus TSCHUDI 1845.

Ptychohyla TAYLOR 1944: Amph. ANOUR. Microhylid.

Ptycholepis RICHARDSON 1843 : P.-CYPRINIF. (Characoid.) Characid.

Ptychophis GOMES 1915 : R.-SQUAMAT. (Serpent.) Boigid. 
Ptychostomus A G A S I Z 1855: P.-CYPRINIF. (Cyprinoid.) Catostomid.

Ptychorhina PETERS 1871: syn. d'Hipposideros GRAY 1831.

Ptychozoon KUHL 1822 : R.-SQUAMAT. (Saur.) Geckonid.

Ptyctolaemus PETERS 1865 : R.SQUAMAT. (Saur.) Agamid.

Ptyobranchus Mc CLELLAND 1844: P. - ANGUILLIF. (Anguilloid.) Moringuid.

Ptyodactylus OKEN 1817: R.-SQUAMAT. (Saur.) Geckonid.

Ptyonoprogne REICHENBACH 1850: Av. PASSERIF. (Acromyod.) Hirundinid.

Ptyonotus GUN T H E R 1860 : P.PERCIF. (Cottoid.) Cottid.

Pucherhamphus MATHEWS 1935 : syn. de Pygoscelis WAGLER 1832.

Pucrasia GRAY 1841: Av. PHASIANIF. Phasianid. (Phasianin.).

Pudella THOMAS 1913 : M.-PARAX. Cervid.

Pudu GRAY 1850 : M.-PARAX. Cervid.

Puffinaria LESSON 1828: Av. PELECANOIDIF. Pelecanoidid.

Puffinus BRISSON 1760 : Av. PROCELLARIIF. Procellariid.

Pullomys SODY 1941 : M.-RODENT. (Myomorph.) Murid. (Murin.).

Pulsatrix KAUP 1848: Av. STRIGIF. Strigid. (Strigin.).

Puma JARDINE 1834: s./g. de Felis LINNE 1758.

Punanetta BONAPARTE 1856: s./g. de Peocilonetta STRICKLAND 1842.

Pungnaso WHITLEY 1948 : P.SYNGNATHIF. Syngnathid.

Pungitius ANNONE 1760 : syn. de Pygosteus GILL 1861.

Pungtungia HERTZENSTEIN 1892: P.-CYPRINIF. (Cyprinoid.) Cyprinid.

Puntazzo BLEEKER 1876 : P.-PERCIF. (Percoid.) Sparid.

Puntioplites SMITH 1929: P.-CYPRINIF. (Cyprinoid.) Cyprinid.
Puntius HAMILTON 1822 : P.-CYPRINIF. (Cyprinoid.) Cyprinid.

Purpureicephalus BONAPARTE 1854: Av. PSITTACIF. Psittacid.

Pusa SCOPOLI 1777 : s./g. de Phoca LINNE 1758.

Pusichthys SWAINSON 1838 : P.CYPRINIF. (Siluroid.) Schilbeid.

Putorius FRISCH 1775: syn. de Mustela LINNE 1758.

Putorius CUVIER 1817: s./g. de Mustela LINNE 1758.

Pycnochromis FOWLER 1941 : P.PERCIF. (Labroid.) Pomacentrid.

Pycnocraspedon ALCOCK 1889: P.-PERCIF. (Ophidioid.) Brotulid.

Pycnomma RUTTER 1904 : P.-PERCIF. (Gobioid.) Gobiid.

Pycnonotus BOIE 1826 : Av. PAS SERIF. (Acromyod.) Pycnonotid.

Pycnoptilus GOULD 1854: Av. PASSERIF. (Acromyod.) Timaliid.

Pycnopyoius SALVADORI 1881 : Av. PASSERIF. (Acromyod.) Meliphagid.

Pycnorhamphus HUME 1874 : Av. PASSERIF. (Acromyod.) Fringillid. (Coccothraustin.).

Pyctoris HODGSON 1844 : Av. PASSERIF. (Acromyod.) Timaliid.

Pygarrhichas BURMEISTER 1837 : Av. PASSERIF. (Mesomyod.) Dendrocolaptid.

Pygathrix GEOFFROY-ST-HILAIRE 1812: s./g. de Semnopithecus CUVIER 1821.

Pygeretmus GOLGER 1841: M.RODENT. (Myomorph.) Dipodid. (Dipodin.).

Pygidianops MYERS 1944 : P.-CYPRINIF. (Siluroid.) Trichomycterid.

Pygidium MEYEN 1834: syn. de Trichomycterus VALENCIENNES 1833 .

Pygiptila SCLATER 1858 : Av. PASSERIF. (Mesomyod.) Formicariid.

Pygmaeus SHERBON 1902 : syn. de Pongo de LACEPEDE 1799. 
Pygmornis BONAPARTE 1854:Av. APODIF. Trochilid.

Pygmura ANDERSON 1873: syn. d'Anourosorex MILNE-EDWARDS 1870.

Pygocentrus MULLER et TROSCHEL 1844: P.-CYPRINIF. (Characoid.) Characid.

Pygochelidon BAIRD 1865: Av. PASSERIF. (Acromyod.) Hirundinid.

Pygoderma PETERS 1865 : M.-CHIROPT. (Microchiropt.) Phyllostomatid. (Stenodermin.).

Pygomeles GRANDIDIER 1867 : R.SQUAMAT. (Saur.) Scincid.

Pygoplites FRASER-BRUNNER 1933: P. - PERCIF. (Percoid.) Chaetodontid.

Pygopristis MULLER et TROSCHEL 1844: P. - CYPRINIF. (Characoid.) Characid.

Pygoptila CABANIS et HEINE 1860: Av. PASSERIF. (Mesomyod.) Formicariid. (Thamnophilin.).

Pygopus MERREM 1820 : R.-SQUAMAT. (Saur.) Pygopodid.

Pygoscelis WA GLER 1832: Av. SPHENISCIF. Spheniscid.

Pygosteus GILL 1861: P.-GASTEROSTEIF. Gasterosteid.

Pylodictis RAFINESQUE 1819 : P.CYPRINIF. (Siluroid.) Bagrid.

Pyramodon SMITH et RADCLIFFE 1913 : P.-PERCIF. (Ophidioid.) Brotulid.

Pyranga VIEILLOT 1816 : Av. PASSERIF. (Acromyod.) Tanagrid.

Pyrenestes SWAINSON 1837: Av. PASSERIF. (Acromyod.) Ploceid. (Passerin.).

Pyrgilauda BONAPARTE 1850: Av. PASSERIF. (Acromyod.) Ploceid. (Passerin.).

Pyrgitopsis BONAPARTE 1850:Av. PASSERIF. (Acromyod.) Ploceid.

Pyriglena CABANIS 1847 : Av. PASSERIF. (Mesomyod.) Formicariid. (Formicariin.).

Pyrocephalus GOULD 1839: Av. PASSERIF. (Mesomyod.) Tyrannid. (Fluvicolin.).
Pyroderus GRAY 1840: Av. PASSERIF. (Mesomyod.) Cotingid.

Pyrofelis GRAY 1874 : syn. de Felis LINNE 1758.

Pyromelana BONAPARTE 1831 : Av. PASSERIF. (Acromyod.) Ploceid. (Estrildin.).

Pyromys THOMAS 1911: M.-RODENT. (Myomorph.) Murid. (Murin.).

Pyrotrogon BONAPARTE 1854: Av. TROGONIF. Trogonid.

Pyrrherodia FINSCH et HARTLAUB 1870 : syn. d'Ardea LINNE 1758.

Pyrrhocheira REICHENBACH 1850: Av. PASSERIF. (Acromyod.) Sturnid. (Graculin.).

Pyrrhococcyx CABANIS 1846: Av. CUCULLIF. Cucullid.

Pyrrhocoma CABANIS 1851: Av. PASSERIF. (Acromyod.) Tanagrid.

Pyrrhocorax TUNSTALL 1771 nec VIEILLOT 1816: Av. PASSERIF. (Acromyod.) Corvid.

Pyrrhoglaux MARQUIS et YAMASHINA 1938 : syn. de Otus PENNANT 1769.

Pyrrholaemus GOULD 1841: Av. PASSERIF. (Acromyod.) Sylviid.

Pyrrhomyias CABANIS et HEINE 1860: Av. PASSERIF. (Mesomyod.) Tyrannid.

Pyrrhoplectes HODGSON 1844: Av. PASSERIF. (Acromyod.) Fringillid. (Fringillin.).

Pyrrhospiza HODGSON 1845 : Av. PASSERIF. (Acromyod.) Fringillid. (Fringillin.).

Pyrrhula BRISSON 1760 : Av. PASSERIF. (Acromyod.) Fringillid. (Fringillin.).

Pyrrhulauda SMITH 1837: Av. PASSERIF. (Acromyod.) Alaudid.

Pyrrhulina CUVIER et VALENCIENNES 1846 : P.-CYPRINIF. (Characoid.) Characid.

Pyrrhulopsis REICHENBACH 1850 : Av. PSITTACIF. Psittacid.

Pyrrhulorhyncha GIGLIOLI 1865 : Av. PASSERIF. (Acromyod.) Fringillid. (Emberizin.). 
Pyrrhuloxia BONAPARTE 1850 : Av. PASSERIF. (Acromyod.) Fringillid. (Fringillin.).

Pyrrhuphonia BONAPARTE 1850 : Av. PASSERIF. (Acromyod.) Tanagrid.

Pyrrhura BONAPARTE 1856: Av. PSITTACIF. Psittacid.

Pyrrhurus CASSIN 1859: Av. PASSERIF. (Acromyod.) Pycnonotid.

Python DAUDIN 1803: R.-SQUAMAT. (Serpent.) Boid. (Pythonin.).

Pythonichthys POEY 1867 : P.-ANGUILLIF. (Anguilloid.) Muraenid.

Pytilia SWAINSON 1837: Av. PASSERIF. (Acromyod.) Ploceid.

Pyxicephalus TSCHUDI 1838: Amph. ANOUR. Ranid.

Pyxis BELL 1827 : R.-TESTUDIN. (Thecoph.) Testudinid.

\section{$\mathbf{Q}$}

Quadrarius JORDAN 1907: P.-PERCIF. (Percoid.) Lutjanid.

Quassilabia JORDAN et BRAYTON 1878: P.-CYPRINIF. (Cyprinoid.) Catostomid.

Quassiremus JORDAN 1892 : P.ANGUILLIF. (Congroid.) Ophichthyid.

Quedenfeltia BOETTEGER 1883 : R.-SQUAMAT. (Saur.) Geckonid.

Quelea REICHENBACH 1850: Av. PASSERIF. (Acromyod.) Ploceid. (Euplectin.).

Quenselia JORDAN et GROSS 1888 : P.-PLEURONECTIF. (Soleoid.) Soleid.

Querigalaxias WHITLEY 1935 : P.$C L U P E I F$. (Galaxioid.) Galaxiid.

Querimana JORDAN 1883 : P.-MUGILIF. Mugilid.

Querquedula OKEN 1817: Av. ANATIF. Anatid. (Anatin.),

Quesiparens IREDALE 1948: Av. PASSERIF. (Acromyod.) Paradiseid. (Paradisein.).

Querula VIEILLOT 1816 : Av. PASSERIF. (Mesomyod.) Cotingid.
Quietula JORDAN et EVERMANN 1895 : P.-PERCIF. (Gobioid.) Gobiid.

Quincuncia GILBERT et HUBBS 1920 : P.-GADIF. Macrurid.

Quintana HUBBS 1934 : P.-CYPRINODONTIF. Poeciliid.

Quinquarius JORDAN 1907 : P.PERCIF. (Percoid.) Lutjanid.

Quirichthys WHITLEY 1951: P.MUGILIF. Atherinid.

Quiris WHITLEY 1950: P.-CLUPEIF. (Stomiatoid.) Melanostomiatid.

Quiscalus VIEILLOT 1816: Av. PASSERIF. (Acromyod.) Icterid. (Quiscalin.).

Quisquilius JORDAN et EVERMANN 1903: P.-PERCIF. (Gobioid.) Gobiid.

Ouoyornis MATHEWS 1912: Av. PASSERIF. (Acromyod.) Laniid. ou Muscicapid.

\section{$\mathbf{R}$}

Ra WHITLEY 1931 : P.-CYPRINIF. (Characoid.) Characid.

Rabdophorus SWAINSON 1839 : P.-PERCIF. (Percoid.) Chaetodontid.

Rabidus JORDAN et EVERMANN 1896 : syn. de Schilbeodes BLEEKER 1858.

Rabirubia JORDAN et FESLER 1893 : P. - PERCIF. (Percoid.) Lutjanid.

Rabula JORDAN et DAVIS 1892 : P. - ANGUILLIF. (Anguilloid.) Muraenid.

Rachicentron: vide Rachycentron KAUP 1826.

Rachidelus : vide Rhachidelus BOULENGER 1908.

Rachinotus : vide Rhachinotus CANTOR 1850.

Rachiodon JOURDAN 1833 : syn. de Dasypeltis WAGLER 1830.

Rachovia MYERS 1927 : P.-CYPRINODONTIF. Cyprinodontid.

Rachoviscus MYERS 1926 : P.-CYPRINIF. (Characoid.) Characid.

Rachycentron KAUP 1826 : P.-PERCIF. (Percoid.) Carangid. ou Rachycentrid. 
Racoma Mc CLELLAND et GRIFFITH 1842 : P.-CYPRINIF. (Cyprinoid.) Cyprinid.

Raconda GRAY 1831 : P.-CLUPEIF. (Clupeoid.) Clupeid.

Racovitzia DOLLO 1900 : P.-PERCIF. (Trachinoid.) Nototheniid.

Radinulopsis SOLDATOV et LINDBERG 1930 : P.-PERCIF. (Cottoid.) Cottid.

Radjah REICHENBACH 1852: Av. ANATIF. Anatid. (Anatin.).

Radulinellus BOLIN $1950: s . / g$. de Radulinus GILBERT 1890.

Radulinus GILBERT 1890 : syn. de Prionistius BEAN 1884.

Rafinesquiellus JORDAN et EVERMANN 1896 : P.-PERCIF. (Percoid.) Etheostomid.

Raia : vide Raja LINNE 1758.

Raiamas JORDAN 1919 : P.-CYPRINIF. (Cyprinoid.) Cyprinid.

Raizero JORDAN et FESLER 1893 : P.-PERCIF, (Percoid.) Lutjanid.

Raja LINNE 1758 : P.-RAJIF. (Rajoid.) Rajid.

Rallicula SCHLEGEL 1871: Av. RALLIF. Rallid.

Rallina GRAY 1846 : Av. RALLIF. Rallid.

Rallus LINNE 1758: Av. RALLIF. Rallid.

Rama BLEEKER 1858 : P.-CYPRINIF. (Siluroid.) Bagrid.

Ramanella RAO 1925: Amph. ANOUR. Microhylid.

Rambaibarnia FOWLER 1934 : P.CYPRINIF. (Cyprinoid.) Cyprinid.

Ramnhocephalus : vide Rhamphocelus DESMAREST 1805.

Ramphocottus : vide Rhamphocottus GUNTHER 1874.

Ramsayornis MATHEWS 1912 : Av. PASSERIF. (Acromyod.) Meliphagid.

Ramularia JORDAN et EVERMANN 1898: P.-PLEURONECTIF. (Pleuronectoid.) Bothid.

Rana LINNE 1758 : Amph. ANOUR. Ranid.

Ranchovia MYERS 1927 : P.-CYPRINODONTIF. Cyprinodontid.
Randia DELACOUR et BERLIOZ 1931: Av. PASSERIF. (Acromyod.) Sylviid.

Rangifer FRISCH 1775 : nom. nud. syn. de Rangifer SMITH 1827.

Rangifer SMITH 1827 : M.-PARAX. Cervid.

Raniceps OKEN 1817 : P.-GADIF. Gadid.

Ranidens BOULENGER 1882 : syn. de Ranodon KESSLER 1866.

Ranodon KESSLER 1866 : Amph. UROD. Hynobiid. ou Ambystomatid.

Ranosoma AHL 1924 : syn. de Rana LINNE 1758.

Ranulina JORDAN et STARKS 1906: P.-PERCIF. (Gobioid.) Gobiid.

Ranzania NARDO 1840 : P.TETRAODONTIF. (Moloid.) Molid.

Raogobius M U K ER J I 1935 : P.PERCIF. (Gobioid.) Gobiid.

Raphicerus SMITH 1827: M.-PARAX. Bovid. (Raphicerin.).

Rhaphiobelone : P.-BELONIF. (Belonoid.) Belonid.

Raphiodonichthys : P. - CYPRINIF. (Characoid.) Characid.

Raphiolepis : P.-PERCIF. (Percoid.). Carangid.

Raphipterus GRAY 1844 : syn. de Merganetta GOULD 1842.

Raphus MOHRING 1758: Av. COLUMBIF. Columbid. (Raphin.) (éteint).

Rappia GUNTHER 1865: Amph. ANOUR. Rhacophorid.

Rarotonga: vide Rorotonga MATHEWS 1925.

Rasbora BLEEKER 1860: P.-CYPRINIF. (Cyprinoid.) Cyprinid.

Rasborichthys BLEEKER 1859 : P.CYPRINIF. (Cyprinoid.) Cyprinid.

Rasborinus OSHIMA 1920 : P.-CYPRINIF. (Cyprinoid.) Cyprinid.

Rastelliger JORDAN et STARKS 1908 : P.-PERCIF. (Scombroid.) Scombrid.

Rastrineobola FOWLER 1936 : s./g. d'Engraulicypris GUNTHER 1894. 
Rastrinus JORDAN et EVERMANN 1896: P. - PERCIF. (Cottoid.) Cottid.

Rataboura GRAY 1831: P.-ANGUILLIF. (Anguilloid.) Moringuid. ou Echelid.

Ratabulus JORDAN et HUBBS 1925 : P. - PERCIF. (Scorpaenoid.) Platycephalid.

Ratellus GRAY 1827 : syn. de Mellivora STORR 1780.

Rattus F I S C HE R 1803 : M.-RODENT. (Myomorph.) Murid. (Murin.).

Ratufa GRAY 1867: M.-RODENT. (Sciuromorph.) Sciurid. (Sciurin.).

Rectes REICHENBACH 1850: Av. PASSERIF. (Acromyod.) Prionopid.

Rectoris YIN 1935 : P.-CYPRINIF. (Cyprinoid.) Cyprinid.

Recurvirostra LINNE 1758: Av. CHARADRIIF. Charadriid. (Recurvirostrin.).

Redigobius HERRE 1927 : P.-PERCIF. (Gobioid.) Eleotrid.

Redunca SMITH 1827 : M.-PARAXON. Bovid. (Reduncin.).

Regalecus ASCANIUS 1772: P.LAMPRIDIF. Trachypterid.

Reganella EIGENMANN 1905 : P.CYPRINIF. (Siluroid.) Loricariid.

Regania JORDAN et STARKS 1904 : P.-GADIF. Macrurid.

Reganichthys BIGELOW et BARBOUR 1944 : P.-PERCIF. (Percoid.) Carangid.

Reganichthys OGILBY 1915 : syn. de Reganula BIGELOW 1944.

Reganina FOWLER 1907: P.-CYPRINIF. (Characoid.) Characid.

Reganisalanx FANG 1934 : P.-CLUPEIF. (Salmonoid.) Salangid.

Reganula BIGELOW 1944 : P.-LOPHIIF. (Ceratoid.) Ceratiid.

Regificola WHITLEY 1931: P.PERCIF. (Percoid.) Carangid.

Regilophotes WHITLEY 1933 : P.LAMPRIDIF. Lophotid.

Reguloides BLYTH 1847 : s./g. de Phylloscopus BOIE 1826.
Regulus BARTRAM 1791 : Av. PASSERIF. (Acromyod.) Regulid.

Reighardina CREASER et HUBBS 1922 : Cycl. PETROMYZONIF. Petromyzonid.

Reinarda HARTERT 1915: Av. APODIF. Apodid.

Reinholdia MATHEWS 1912: Av. PROCELLARIIF. Procellariid.

Reinwardtia TEMMINCK:Av. TROGONIF. Trogonid.

Reinwardtoenas $B O N A P A R T E$ 1854 : Av. COLUMBIF. Columbid. (Columbin.).

Reithrodon WATERHOUSE 1837 : M.-RODENT. (Myomorph.) Cricetid. (Cricetin. ou Hesperomyin.).

Reithrodontomys GIGLIOLI 1874: M.-RODENT. (Myomorph.) Cricetid. (Cricetin. ou Hesperomyin.).

Reithronycteris MILLER $1890: M$. CHIROPT. (Microchiropt.) Phyllostomatid. (Phyllonycterin.).

Reithrosciurus GRAY 1867 : M.-RODENT. (Sciuromorph.) Sciurid. (Sciurin.).

Relictivomer de CARVALHO 1954 : Amph. ANOUR. Microhylid.

Remilegia GILL 1863 : P.-PERCIF. (Echeneoid.) Echeneid.

Remiz JAROCKI 1819 : Av. PASSERIF. (Acromyod.) Paradiseid. (Paradisein.).

Remiza STEJNEGER 1886: Av. PASSERIF. (Acromyod.) Parid.

Remizus PARAZAK 1897 : syn. de Remiz JAROCKI 1819.

Remora GOUAN 1770 : P.-PERCIF. (Echeneoid.) Echeneid.

Remorina JORDAN et EVERMANN 1896 : P.-PERCIF. (Echeneoid.) Echeneid.

Remoropsis GILL 1863 : P.-PERCIF. (Echeneoid.) Echeneid.

Rendahlia CHABANAUD 1930 : P.PLEURONECTIF. (S ole o $i d$.) Soleid.

Reniceps GILL 1861: P.-GALEIF. (Carcharhinoid.) Sphyrnid.

Repomucenus WHITLEY 1931 : P.PERCIF. (Callionymoid.) Callionymid. 
Reporhamphus WHITLEY 1931: P.-BELONIF. (Exocoetoid.) Hemirhamphid.

Repotrudis WHITLEY $1930: s . / g$. de Platycephalus BLOCH 1795.

Retroculus EIGENMANN et BRAY 1894: P.-PERCIF. (Percoid.) Cichlid.

Retrovinna GILL 1863: P.-CLUPEIF. (Salmonoid.) Salangid.

Rewa WHITLEY 1950 : P.-PERCIF. (Gobioid.) Gobiid.

Rexea WAITE 1911 : P.-PERCIF. (Trichiuroid.) Gempylid.

Rhabdaethiops FOWLER 1936 : s./g. de Nannaethiops GUNTHER 1872.

Rhabdalestes : P.-CYPRINIF. (Characoid.) Characid.

Rhabdamia WEBER 1909 : P.-PERCIF. (Percoid.) Serranid.

Rhabdoblennius WHITLEY 1930 : P.-PERCIF. (Blennioid.) Blenniid.

Rhabdolichops EIGENMANN 1942 : P.-C Y P RIN I F. (Gymnotoid.) Sternarchid.

Rhabdomys THOMAS 1916 : M.-RODENT. (Myomorph.) Murid. (Murin.).

Rhabdopetersius HOEDEMAN 1951: P.-CYPRINIF. (Characoid.) Characid.

Rhabdophidium BOULENGER 1894 : R.-SQUAMAT. (Serpent.) Colubrid.

Rhabdophis FITZINGER 1843 : R.SQUAMAT. (Serpent.) Colubrid.

Rhabdophorus AGASSIZ 1846 : P.PERCIF. (Percoid.) Chaetodontid.

Rhabdops BOULENGER 1893 : R.SQUAMAT. (Serpent.) Colubrid.

Rhabdornis REICHENBACH 1853 : Av. PASSERIF. (Acromyod.) Certhiid.

Rhabdosargus FOWLER 1933 : s./g. de Diplodus RAFINESQUE 1810.

Rhabdosebastes FOWLER et BEAN 1922: P.-PERCIF. (Percoid.) Serranid.

Rhabdura OGILBY 1907 : P.-ANGUILLIF. (Anguilloid.) Muraenid.
Rhachianectes COPE 1869 : syn. de Eschrichtius GRAY 1864.

Rhachicentron: vide Rachycentron KAUP 1826.

Rhachidelus BOULENGER 1908 : R.-SQUAMAT. (Serpent.) Boigid.

Rhachinotus CANTOR 1850 : P.RAJIF. (Dasyatoid.) Dasyatid.

Rhachiodon: vide Rachiodon JOURDAN 1833.

Rhacochilus AGASSIZ 1854: P.PERCIF. (Labroid.) Embiotocid.

Rhacodactylus FITZINGER 1843 : R.SQUAMAT. (Saur.) Geckonid.

Rhacophorus KUHL et van HASSELT 1822: Amph. ANOUR. Rhacophorid.

Rhadinea COPE 1868 : syn. de Liophis WAGLER 1830.

Rhadinella SMITH 1941 : R.-SQUAMAT. (Serpent.) Colubrid.

Rhadinesthes REGAN et TREWAVAS 1929 : P.-CLUPEIF. (Stomiatoid.) Astronesthid.

Rhadinocentrus REGAN 1914 : P.MUGILIF. Atherinid.

Rhadinophis VOGT 1922: R.SQUAMAT. (Serpent.) Colubrid.

Rhagologus STRESEMANN et PALUDAN 1934: Av. PASSERIF. (Acromyod.) Laniid.

Rhagomys THOMAS 1917 : M.-RODENT. (Myomorph.) Cricetid. (Cricetin. ou Hesperomyin.).

Rhamdella EIGENMANN et EIGENMANN 1888: P.-CYPRINIF. (Siluroid.) Bagrid.

Rhamdioglanis IHERING 1907 : P.CYPRINIF. (Siluroid.) Bagrid.

Rhamdiopsis HASEMANN 1911 : P.-CYPRINIF. (Siluroid.) Bagrid.

Rhamnophis GUNTHER 1862 : R.SQUAMAT. (Serpent.) Colubrid.

Rhamphalcyon REICHENBACH 1851 : Av. CORACIADIF. Alcedinid.

Rhamphastos LIN NE 1758: Av. PICIF. Rhamphastid. 
Rhamphichthys MULLER 1845 : P. CYPRINIF. (Gymnotoid.) Sternarchid.

Rhamphiculus CAIN $1954:$ s./g. de Ptilinopus SWAINSON 1825.

Rhamphiophis PETERS 1854: R.SQUAMAT. (Serpent.) Boigid.

Rhamphobatis GILL 1862 : P.-RAJIF. (Rhinobatoid.) Rhinobatid.

Rhamphoberyx GILL 1863 : P.-BERYCIF. Holocentrid.

Rhamphocoenus VIEILLOT 1819 : Av. PASSERIF. (Mesomyod.) Formicariid.

Rhamphocephalus : $v i d e$ Rhamphocelus DESMAREST 1805.

Rhamphocharis SALVADORI 1875: Av. PASSERIF. (Acromyod.) Drepaniid.

Rhamphochromis REGAN 1922: P.-PERCIF. (Percoid.) Cichlid.

Rhamphocinclus de LAFRESNAYE 1843: Av. PASSERIF. (Acromyod.) Mimid.

Rhamphococcyx CABANIS et HEINE 1863: Av. CUCULLIF. Cucullid.

Rhamphocelus DESMAREST 1805 : Av. PASSERIF. (Acromyod.) Tanagrid.

Rhamphocorys BONAPARTE 1854 : Av. PASSERIF. (Acromyod.) Alaudid.

Rhamphocottus GUNTHER 1874: P.-PERCIF. (Cottoid.) Cottid.

Rhamphodermogenys FOWLER et $B E A N$ 1922: P.-BELONIF. (Exocoetoid.) Hemirhamphid.

Rhamphodon LESSON 1830: Av. APODIF. Trochilid.

Rhampholeon GUNTHER 1874: syn. de Brookesia GRAY 1864.

Rhamphomantis SALVADORI 1878: Av, CUCULLIF, Cucullid.

Rhamphomicron BONAPARTE 1850: Av. APODIF. Trochilid.

Rhamphosternarchus GUNTHER 1870 : P.-CYPRINIF. (Gymnotoid.) Sternarchid.

Rhamphostruthus MAYR 1931: s. g. d'Erythrura SWAINSON 1837.

Rhamphotrigon SNETHLAGE
1914: Av. PASSERIF. (Mesomyod.) Tyrannid.

Rhamphozosterops MAYR 1931 : syn. de Rukia MOMIYAMA 1922.

Rhaphiobelone FOWLER 1934 : P.BELONIF. (Belonoid.) Belonid.

Rhaphiodon AGASSIZ 1829 : P.-CYPRINIF. (Characoid.) Characid.

Rhea BRISSON 1760: Av. STRUTHIONIF. Rheid.

Rhechias JORDAN 1921: P.-ANGUILLIF. (Congroid.) Congrid.

Rhectes ROSENBERG 1864: emend. pro $R$ e ctes REICHENBACH 1850.

Rhectogramma NORMAN 1930 : P. PERCIF. (Percoid.) Serranid.

Rhegma JORDAN et EVERMANN 1900 : syn. de Pseudogramma BLEEKER 1875.

Rhegmatorhina RIDGWAY 1888 : Av. PASSERIF. (Mesomyod.) Formicariid.

Rheinhardtius OGILVIE-GRANT 1893: Av. PHASIANIF. Phasianid. (Phasianin.).

Rheithrosciurus : vide Reithrosciurus GRAY 1867.

Rhencus JORDAN et EVERMANN 1896 : P. - PERCIF. (Percoid.) Lutjanid.

Rheocles JORDAN 1919 : P.-MUGILIF. Atherinid.

Rheocloides NICHOLS et LAMONTE 1931: P.-MUGILIF. Atherinid.

Rheocrypta J ORDAN 1877 : P.PERCIF. (Percoid.) Etheostomid.

Rheomys THOMAS 1906: M.-RODENT. (Myomorph.) Cricetid. (Cricetin. ou Hesperomyin.).

Rheopresbe JORDAN et STARKS 1904: P.-PERCIF. (Cottoid.) Cottid.

Rhesus LESSON 1840 : nom. nud. syn. de Macaca de LACEPEDE 1799.

Rhina WALBAUM 1792: syn. de Squatina DUMERIL 1806.

Rhina SCHAEFFER 1760 : P.-RAJIF. (Rhinobatoid.) Rhinobatid.

Rhinatrema DUMERIL et BIBRON 1841 : Amph. APOD. Caeciliid. 
Rhincodon SMITH 1829: P.-GALEIF. (Isuroid.) Rhincodontid.

Rhinecanthus SWAINSON 1839: P.-PERCIF. (Balistoid.) Balistid.

Rhinechidna BARBOUR 1908: P.ANGUILLIF. (Anguilloid.) $\mathrm{Mu}$ raenid.

Rhinechis MICHAHELLES 1832 : R.-SQUAMAT. (Serpent.) Colubrid.

Rhinecodon AGASSIZ 1845: emend. pro Rhincodon SMITH 1829.

Rhinelaps GUNTHER 1872: R.SQUAMAT. (Serpent.) Elapid.

Rhinelepis SPIX 1829 : P.-CYPRINIF. (Siluroid.) Loricariid.

Rhineloricaria : P.-CYPRINIF. (Siluroid.) Loricariid.

Rhinemys WAGLER 1830 : R.-TESTUDIN. (Thecoph.) Chelydid.

Rhineodon $S$ WAINSON 1839 : emend. pro Rhincodon SMITH 1829.

Rhinemosus SWAINSON 1839 : P.TETRAODONTIF. (Ostracionoid.) Ostracionid.

Rhineura COPE 1861: R.-SQUAMAT. (Saur.) Amphisbaenid.

Rhinhoplocephalus BOULENGER 1896 : emend. pro Rhinoplocephalus MULLER 1885.

Rhinichthys AGASSIZ 1849 : P.-CYPRINIF. (Cyprinoid.) Cyprinid. ou Catostomid.

Rhinobagrus BLEEKER $1865: s . / g$. de Liocassis GUNTHER 1864.

Rhinobates SWAINSON 1838 : vide Rhinobatos LINCK 1790.

Rhinobatis de BLAINVILLE 1825 : vide Rhinobatos LINCK 1790.

Rhinobatos LINCK 1795: P.-RAJIF. (Rhinobatoid.) Rhinobatid.

Rhinobatus WALBAUM 1792 : vide Rhinobatos LINCK 1790.

Rhinoberyx GILL 1862: P.-BERYCIF. Holocentrid.

Rhinobothryum WAGLER 1830 : R.-SQUAMAT. (Serpent.) Boigid.

Rhinobrycon MYERS 1944 : P.-CYPRINIF. (Characoid.) Characid.
Rhinocalamus GUNTHER 1888 : R.-SQUAMAT. (Serpent.) Boigid.

Rhinoceros LINNE 1758: M.-PERISSODACT. (Ceratomorph.) Rhinocerotid. (Rhinocerotin.).

Rhinocheilus BAIRD et GIRARD $1853: R$.-

Rhinochetos VERREAUX et des MURS 1860 : Av. RALLIF. Rhinochetid.

Rhinochimaera GARMAN 1901 : P. HOLOCEPH. Rhinochimaerid.

Rhinochoerus WAGLER 1830 : syn. de Tapirus BRISSON 1762.

Rhinocichla SHARPE 1883: Av. PASSERIF. (Acromyod.) Paradoxiornithid.

Rhinococcyx SHARPE 1873: Av. CUCULLIF. Cucullid.

Rhinocorax SHARPE 1877: Av. PASSERIF. (Acromyod.) Corvid.

Rhinocrepis GERVAIS 1836: syn. de Rhinolopus de LACEPEDE 1799.

Rhinocrypta GRAY 1841 : Av. PASSERIF. (Mesomyod.) Pteroptochid. (Rhinocryptin.).

Rhinocryptis PETERS 1844: syn. de Protopterus OWEN 1837.

Rhinodactylus SMITH 1957 : P.PERCIF. (Acanthuroid.) Acanthurid.

Rhinodelphis WAGNER 1846 : syn. de Delphinus LINNE 1758.

Rhinoderma DUMERIL et BIBRON 1841: Amph. ANOUR. Microhylid.

Rhinodon SMITH 1839: syn. de Rhincodon SMITH 1829.

Rhinodoras BLEEKER 1862: P.CYPRINIF. (Siluroid.) Doradid.

Rhinodryas WERNER 1903: R.SQUAMAT. (Serpent.) Boigid.

Rhinogale GRAY 1865: s./g. de Rhynchogale THOMAS 1894.

Rhinoglanis GUNTHER 1864: P.CYPRINIF. (Siluroid.) Loricariid.

Rhinogobio BLEEKER 1870 : P.CYPRINIF, (Cyprinoid.) Cyprinid.

Rhinogobioides RENDHAL 1928 : 
syn. de Rhinogobio BLEEKER 1870.

Rhinogobiops $H U B B S 1926$ : P.PERCIF. (Gobioid.) Gobiid.

Rhinogobius GILL 1868 : P.-PERCIF. (Gobioid.) Gobiid.

Rhinoliparis GILBERT 1895: P. PERCIF. (Cottoid.) Cyclopterid.

Rhinolophus de LACEPEDE 1799 : M. -CHIROPT. (Microchiropt.) Rhinolophid. (Rhinolophin.).

Rhinomegalophus BOURRET 1951: M. - CHIROPT. (Microchiropt.) Rhinolophid.

Rhinomugil GILL 1864 : P.-MUGILIF. Mugilid.

Rhinomuraena GARMAN 1889 : P.ANGUILLIF. (Anguilloid.) $\mathrm{Mu}$ raenid.

Rhinomyias SHARPE 1879: Av. PASSERIF. (Acromyod.) Muscicapid.

Rhinonemus GILL 1864: P.-GADIF. Gadid.

Rhinonycteris GRAY 1866 : M.-CHIROPT. (Microchiropt.) Hipposiderid.

Rhinophis HEMPRICH 1830 : R.SQUAMAT. (Serpent.) Uropeltid.

Rhinophrynus DUMERIL et BIBRON 1841: Amph. ANOUR. Bufonid.

Rhinophylla PETERS 1865: M.CHIROPT. (Microchiropt.) Phyllostomatid. (Carolliin.).

Rhinophylla GRAY 1866: syn. d'Hipposideros GRAY 1831.

Rhinophyllotis IREDALE et TROUGHTON 1934: M.-CHIROPT. (Microchiropt.) Rhinolophid.

Rhinopias GILL 1905 : P.-PERCIF. (Scorpaenoid.) Synancejid.

Rhinopithecus MILNE-EDWARDS 1870: M.-PRIMAT. (Simioid.) Cercopithecid. (Colobin.).

Rhinoplagusia BLEEKER 1870 : P.PLEURONECTIF. (Pleuronectoid.) Pleuronectid.

Rhinoplax GLOGER 1842 : Av. CORACIADIF. Bucerotid.

Rhinoplichthys : P.-PERCIF. (Scorpaenoid.) Scorpaenid.
Rhinoplocephalus MULLER 1885 : R.-SQUAMAT. (Serpent.) Elapid.

Rhinopoma GEOFFROY-ST-HILAIRE 1812 : M.-CHIROPT. (Microchiropt.) Rhinopomatid.

Rhinopomastus JARDINE 1828: Av. CORACIADIF. U р и $i d$. (Phoeniculin.).

Rhinopsar DAVIS 1938: Av. PASSERIF. (Acromyod.) Sturnid. (Graculin.).

Rhinoptera CUVIER 1829 : P.-RAJIF. (Myliobatoid.) Rhinopterid.

Rhinopterus MILLER 1906: M. CHIROPT. (Microchiropt.) Vespertilionid. (Vespertilionin.).

Rhinoptilus STRICKLAND 1850 : Av. CHARADRIIF. Glareolid.

Rhinoptynx KAUP 1851: Av. STRIGIF. Strigid. (Bubonin.).

Rhinoraja : P.-RAJIF. (Rajoid.) Rajid.

Rhinorta VIGORS 1830: Av. CUCULLIF. Cucullid.

Rhinosardinia EIGENMANN 1912 : P.-CLUPEIF. (Clupeoid.) Clupeid.

Rhinoscion GILL 1861 : P.-PERCIF. (Percoid.) Sciaenid.

Rhinosciurus GRAY 1843: nom. nud. pro Rhinosciurus BLYTH 1855.

Rhinosciurus BLYTH 1855 : M.-RODENT. (Sciuromorph.) Sciurid. (Sciurin.).

Rhinoscopelus LUTKEN 1892 : P.CLUPEIF. (Myctophoid.) Myctophiid.

Rhinoscymnus GILL 1865 : P.SQUALIF. (Squaloid.) Scymnorhinid.

Rhinosimus DUMERIL 1853: R.SQUAMAT. (Serpent.) Boigid.

Rhinosolea F $O W L E R$ 1946 : P.PLEURONECTIF. (Soleo $i d$.) Soleid.

Rhinosomus : P.-TETRAODONTIF. (Ostracionoid.) Ostracionid.

Rhinostoma FITZINGER 1826 : R.SQUAMAT. (Serpent.) Boigid.

Rhinotriacis GILL 1863 : P.-GALEIF. (Carcharhinoid.) Carcharhinid. 
Rhipidomys TSCHUDI 1844: M.RODENT. (Myomorph.) Cricetid. (Hesperomyin.).

Rhipidornis SALVADORI 1877 : Av. PASSERIF. (Acromyod.) Paradiseid. (Paradisein.).

Rhipidura VIGORS et HORSFIELD 1826: Av. PASSERIF. (Acromyod.) Muscicapid.

Rhithrotriton NESTROV 1916 : Amph. UROD. Salamandrid.

Rhizomys GRAY 1831: M.-RODENT. (Hystricomorph.) Rhizomyid.

Rhizoprion OGILBY 1915 : syn. de Rhizoprionodon WHITLEY 1929.

Rhixoprion JORDAN 1861 : P.GALEIF. (Carcharhinoid.) Carcharhinid.

Rhizoprionodon WHITLEY 1929 : P.-GALEIF. (Carcharhinoid.) Carcharhinid.

Rhizosomichthys MILES 1943 : P.CYPRINIF. (Siluroid.) Trichomycterid.

Rhizotera GRAY 1841: Av. PHASIANIF. Phasianid. (Phasianin.).

Rhoadsia FOWLER 1911: P.-CYPRINIF, (Characoid.) Characid.

Rhodeoides THOMINOT 1884 : P.CYPRINIF. (Cyprinoid.) Cyprinid.

Rhodeops FOWLER 1910 : P.-CYPRINIF. (Cyprinoid.) Cyprinid.

Rhodeus AGASSIZ 1832: P.-CYPRINIF. (Cyprinoid.) Cyprinid.

Rhodichthys COLLETT 1879 : P.PERCIF. (Ophidioid.) Brotulid.

Rhodinocichla HARTLAUB 1853 : Av. PASSERIF. (Acromyod.) Mimid.

Rhodona GRAY $1839: s . / g$. de Lygosoma HARDWICKE et GRAY 1827.

Rhodonessa REICHENBACH 1852 : Av. ANATIF. Anatid. (Anatin.).

Rhodopechys CABANIS 1851: Av. PASSERIF. (Acromyod.) Fringillid. (Fringillin.).

Rhodophila JERDON 1863 : syn. de Saxicola BECHSTEIN 1802.
Rhodopis REICHENBACH 1854: Av. APODIF. Trochilid.

Rhodopleuriscus FOWLER 1944 : P.-CYPRINIF. (Cyprinoid.) Cyprinid.

Rhodospingus SHARPE 1888: Av. PASSERIF. (Acromyod.) Fringillid.

Rhodospiza SHARPE 1888: Av. PASSERIF. (Acromyod.) Fringillid. (Fringillin.).

Rhodostethia Mc GILLIVRAY 1842: Av. LARIF. Larid. (Larin.).

Rhodothraupis RIDGWAY 1893 : Av. PASSERIF. (Acromyod.) Fringillid. ou Tanagrid.

Rhogeessa ALLEN 1866: M.-CHIROPT. (Microchiropt.) Vespertilionid. (Vespertilionin.).

Rhombatractus GILL 1879 : P.MUGILIF. Atherinid.

Rhombiscus JORDAN et SNYDER 1901: P.-PLEURONECTIF. (Pleuronectoid.) Bothid.

Rhombochirus GILL 1863 : P.-PERCIF. (Echenoid.) Echeneid.

Rhomboidichthys BLEEKER 1856 : P. - PLEURONECTIF. (Pleuronectoid.) Bothid.

Rhombomys WAGNER 1841: M.RODENT. (Myomorph.) Gerbillid.

Rhombophryne BOETTEGER 1880: Amph. ANOUR. Microhylid.

Rhomboplites GILL 1863 : P.-PERCIF. (Percoid.) Lutjanid.

Rhomboplitoides FOWLER 1918 : P.-PERCIF. (Percoid.) Lutjanid.

Rhomboserranus FOWLER 1943 : P. - PERCIF. (Percoid.) Serranid.

Rhombosolea GUNTHER 1862 : P.PLEURONECTIF. (Pleuronectoid.) Pleuronectid.

Rhombosoma RE GAN 1914 : P.MUGILIF. Atherinid.

Rhombotides WALBAUM 1792 : P.PERCIF. (Acanthuroid.) Acanthurid.

Rhombus WALBAUM 1792: syn. de Scophthalmus RAFINESQUE 1810.

Rhonciscus JORDAN et EVER- 
MANN 1896 : P.-PERCIF. (Percoid.) Lutjanid.

Rhopias CABANIS et HEINE 1860 : syn. de Myrmotherula SCLATER 1858.

Rhopocichla OATES 1889: Av. PASSERIF. (Acromyod.) Timaliid.

Rhopocichla ALLEN 1891 : syn. de Rhopornis RICHEMOND 1902.

Rhopodytes CABANIS et HEINE 1863: syn. de Phoenicophaeus STEPHENS 1815.

Rhopophilus GIGLIOLI et SALVADORI 1870: Av. PASSERIF. (Acromyod.) Sylviid.

Rhopornis RICHEMOND 1902 : Av. PASSERIF. (Mesomyod.) Formicariid.

Rhopoterpe CABANIS 1847: Av. PASSERIF. (Mesomyod.) Formicariid. (Formicariin.).

Rhoptropella HEWITT 1937 : R.SQUAMAT. (Saur.) Geckonid.

Rhoptropus PETERS 1869: R.SQUAMAT. (Saur.) Geckonid.

Rhotoeca JORDAN 1887 : P.-PERCIF. (Percoid.) Etheostomid.

Rhycanthias JORDAN 1921: P.PERCIF. (Percoid.) Serranid.

Rhyacichthys BOULENGER 1901 : P.-PERCIF. (Gobioid.) Eleotrid.

Rhyacophilus KAUP 1829 : syn. de Tringa LINNE 1758.

Rhyacornis BLANFORD 1872 : Av. PASSERIF. (Acromyod.) Turdid.

Rhyacosideron DUNN 1928 : Amph. UROD. Ambystomatid.

Rhyacotriton DUNN 1920: Amph. UROD. Ambystomatid.

Rhynchactis REGAN 1925 : P.-LOPHIIF. (Ceratoid.) Ceratiid.

Rhynchana RICHARDSON 1844: P.-CLUPEIF. (Gonorhynchoid.) Gonorhynchid.

Rhynchelaps BOULENGER 1896 : R.SQUAMAT. (Serpent.) Elapid.

Rhyncherus OGILBY 1907 : P.-LOPHIIF. (Antennarioid.) Antennariid.

Rhynchiscus MILLER 1907: syn. de Rhynchonycteris PETERS 1867.
Rhynchobatus MULLER et HEINE 1837 : P.-RAJIF. (Rhinobatoid.) Rhinobatid.

Rhynchobdella SCHNEIDER 1801: P. - MASTACEMBELIF. Mastacembelid.

Rhynchoceratias REGAN 1925 : P.LOPHIIF. (Ceratoid.) Ceratiid.

Rhynchoceros GLOGER 1842 : syn. de Lophoceros HEMPRICH et EHRENBERG 1833.

Rhynchoconger JORDAN et HUBBS 1925 : P. - ANGUILLIF. (Congroid.) Congrid.

Rhynchocyclus CABANIS et HEINE 1860 : Av. PASSERIF. (Mesomyod.) Tyrannid. (Spizitornin.).

Rhynchocymba JORDAN et HUBBS 1925: P. - ANGUILLIF. (Congroid.) Congrid.

Rhynchocyon PETERS 1847 : M.INSECTIV. Macroscelid. (Rhynchocyonin.).

Rhynchocyon GISTEL 1848: syn. de Macroglossus CUVIER 1824.

Rhynchocypris GUNTHER 1889 : syn. de Phoxinus AGASSIZ 1835.

Rhynchodon NITZSCH $1829: s . / g$. de Falco LINNE 1758.

Rhynchoedura GUNTHER 1867 : R.-SQUAMAT. (Saur.) Geckonid.

Rhynchoelaps JAN 1859: vide Rhynchelaps BOULENGER 1896.

Rhynchofalco BAIRD, BREWER et RIDGWAY $1874: s . / \mathrm{g}$. de Falco LINNE 1758.

Rhynchogadus : P.-GADIF. Gadid.

Rhynchogale THOMAS 1894: M.FISSIP. Viverrid. (Herpestin.).

Rhynchohyalus BARNARD 1925 : P.-CLUPEIF. (Opisthoproctoid.) Argentinid.

Rhyncholestes OSGOOD 1925 : $M$.MARSUP. Caenolestid.

Rhynchomeles THOMAS 1920 : M.MARSUP. Peramelid.

Rhynchomys THOMAS 1895 : M.RODENT. (Myomorph.) Murid. (Rhynchomyin.).

Rhynchonax THOMAS 1912: syn. d'Uropsilus MILNE-EDWARDS 1872. 
Rhynchonvcteris PETERS 1867 : M. - CHIROPT. (Microchiropt.) Emballonurid.

Rhynchopelates FOWLER 1931: s./g. de Pelates CUVIER et VALENCIENNES 1829 .

Rhynchophanes BAIRD 1858: Av. PASSERIF. (Acromyod.) Fringillid.

Rhynchops LINNE 1760: Av. LARIF. Larid. (Rhynchopin.).

Rhynchopsitta BONAPARTE 1854 : Av. PSITTACIF. Psittacid.

Rhynchopsittacus SALVADORI 1891: syn. de Rhynchopsitta BONAPARTE 1854.

Rhynchorhamphus FOWLER 1928: s./g. d'Hemirhamphus CUVIER 1817.

Rhynchortyx OGILVIE-GRANT 1893: Av. PHASIANIF. Phasianid. (Phasianin.).

Rhynchostomias REGAN et TREWAVAS 1930: s./g. d'Eustomias VAILLANT 1888 (FILHOL 1884).

Rhynchostracion FRASER-BRUNNER 1935: P.-TETRAODONTIF. (Ostracionoid.) Ostracionid.

Rhynchostruthus SCLATER 1881: Av. PASSERIF. (Acromyod.) Fringillid. (Fringillin.).

Rhynchosuchus HUXLEY 1859: syn. de Tomistoma MULLER 1846.

Rhynchothraupis BERLIOZ 1939 : Av. PASSERIF. (Acromyod.) Tanagrid.

Rhynchotragus NEUMANN 1905: s./g. de Madoqua OGILBY 1837.

Rhynchotus SPIX 1825 : Av. TINAMIF. Tinamid.

Rhyneptesicus BIANCHI 1916 : syn. d'Eptesicus RAFINESQUE 1820.

Rhynopoma BOWDICH 1821 : vide Rhinopoma GEOFFROY-ST-HILAIRE 1812.

Rhypticus AGASSIZ 1846 : emend. pro Rypticus CUVIER et VALENCIENNES 1829.

Rhytejulis FOWLER et BEAN 1928 : s./g. de Stethojulis GUNTHER 1861.
Rhyticeros REICHENBACH 1849 : Av. CORACIADIF. Bucerotid.

Rhytidoceros CABANIS et HEINE 1860 : emend. pro Rhyticeros REICHENBACH 1849.

Rhytina ILLIGER 1811: M.-SIREN. Rhytinid. (éteint).

Rhytiodus KNER 1858 : P.-CYPRINIF. (Characoid.) Characid.

Rhytipterna REICHENBACH 1850 : Av. PASSERIF. (Mesomyod.) Cotingid.

Ribeirina PARKER 1934: Amph. ANOUR. Microhylid.

Ribeiroa JORDAN 1920: P.-PERCIF. (Cottoid.) Agonid.

Richardsonia STEINDACHNER 1866 : P.-PERCIF. (Percoid.) Lutjanid.

Richardsonius GIRARD 1856: P.CYPRINIF. (Cyprinoid.) Cyprinid.

Richemondena MATHEWS et IREDALE 1918: Av. PASSERIF. (Acromyod.) Fringillid. (Pitylin.).

Richia COKER 1927 : P.-PERCIF. (Percoid.) Etheostomid.

Richiella COKER 1927: syn. de Poecilichthys AGASSIZ 1854.

Rickettia BIANCHI 1916 : s./g. de Myotis KAUP 1829.

Rictugobius KOUMANS 1932 : syn. de Mahidolia SMITH 1932.

Ricuzenius JORDAN et STARKS 1904: P. - PERCIF. (Cottoid.) Cottid.

Ridgwayia STEJNEGER 1883: Av. PASSERIF. (Acromyod.) Turdid.

Ridgwayornis BERTONI 1926: Av. PASSERIF. (Mesomyod.) Tyrannid. (Platyrhinchid.).

Riekertia SMITH 1952 : P.-PERCIF. (Batrachoid.) Batrachoidid.

Rigoon GISTEL 1848 : syn. de Monachus FLEMMING 1822.

Rileyornis MATHEWS 1927: Av. PASSERIF. (Acromyod.) Muscicapid.

Rimator BLYTH 1847 : Av. PASSERIF. (Acromyod.) Timaliid.

Rimicola JORDAN et EVERMANN 1896 : P.-PERCIF. (Gobiesoxoid.) Gobiesocid. 
Rinoctes PARR 1952 : P.-CLUPEIF. (Alepocephaloid.) Alepocephalid.

Riopa GRAY 1839 : R.-SQUAMAT. (Saur.) Scincid.

Rioraja WHITLEY 1939: P.-RAJIF. (Rajoid.) Rajid.

Riparia FORSTER 1817: Av. PASSERIF. (Acromyod.) Hirundinid.

Ripidura CUVIER 1829: syn. de Rhipidura VIGORS et HORSFIELD 1826.

Risor GINSBURG 1933 : P.-PERCIF. (Gobioid.) Gobiid.

Rissa STEPHENS 1826: Av, LARIF. Larid. (Larin.).

Rissola JORDAN et EVERMANN 1896 : P.-PERCIF. (Ophidioid.) Ophidiid.

Ristella GRAY 1839 : R.-SQUAMAT. (Saur.) Scincid.

Rita BLEEKER 1853: P.-CYPRINIF. (Siluroid.) Bagrid.

Riukiuia FOWLER 1946 : P.-PERCIF. (Gobioid.) Eleotrid.

Rivasella : P.-CYPRINIF. (Characoid.) Characid.

Riverina CASTELNAU 1872: P. PERCIF. (Percoid.) Serranid.

Rivulichthys MYERS 1927 : P.-CYPRINODONTIF. Cyprinodontid.

Rivulus POEY 1860 : P.-CYPRINODONTIF. Cyprinodontid.

Robusqualus WHITLEY 1931: P. SQUALIF. (Squalioid.) Scymnorhinid.

Roccus MITCHILL 1814 : P.-PERCIF. (Percoid.) Serranid.

Roeboides GUNTHER 1864 : $P .-C Y$ PRINIF. (Characoid.) Characid.

Roeboexodon GERY 1959 : P.-CYPRINIF. (Characoid.) Characid.

Roestes GUNTHER 1864: P.-CYPRINIF. (Characoid.) Characid.

Rogadius JORDAN et RICHARDSON 1908: P.-PERCIF. (Scorpaenoid.) Platycephalid.

Rogenia CUVIER et VALENCIENNES 1847 : P.-CLUPEIF. (Clupeoid.) Clupeid.

Rogersornis MATHEWS 1912: Av. PASSERIF. (Acromyod.) Paradiseid. (Paradisein.),
Rohita CUVIER et VALENCIENNES 1842 : P.-CYPRINIF. (Cyprinoid.) Cyprinid.

Rohitichthys BLEEKER 1860 : P.CYPRINIF. (Cyprinoid.) Cyprinid.

Rohitodes BLEEKER 1860 : P.-CYPRINIF. (Cyprinoid.) Cyprinid.

Rohtee SYKES 1839 : P.-CYPRINIF. (Cyprinoid.) Cyprinid.

Rohteichthys BLEEKER 1860 : P.CYPRINIF. (Cyprinoid.) Cyprinid.

Rollulus BONNATERRE 1790 : Av. PHASIANIF. Phasianid. (Phasianin.).

Romerolagus MERRIAM 1896 : M.LAGOMORPH. Leporid. (Palaelolagin.).

Romicia GRAY 1838 : syn. de Pipistrellus KAUP 1829.

Roncador JORDAN 1881 : P.-PERCIF. (Percoid.) Sciaenid.

Ronchifex GISTEL 1848 : P.-PERCIF. (Labroid.) Labrid.

Rondeletia GOODE et BEAN 1894 : P.STEPHANOBERYCIF, Rondeletiid.

Rooseveltiella EIGENMANN 1915: P.-CYPRINIF. (Characoid.) Characid.

Roraimia CHAPMAN 1929: Av. PASSERIF. (Mesomyod.) Dendrocolaptid.

Rorotonga MATHEWS 1925 : syn. de Pomarea BONAPARTE 1854.

Rorqual CUVIER 1829: syn. de Balaenoptera de LACEPEDE 1804.

Rosanthias TANAKA 1918: P.PERCIF. (Percoid.) Serranid.

Rosaura TUCKER 1954: P.-CLUPEIF. (Salmonoid.) Rosaurid.

Rosicola JORDAN et EVERMANN 1896 : P.PERCIF. (Scorpaenoid.) Scorpaenid.

Rosmarus BRUNNICH 1772: syn. d'Odobenus BRISSON 1762.

Rostramnus LESSON 1831: vide Rostrhamnus LESSON 1830.

Rostratula VIEILLOT 1816: Av. CHARADRIIF. Charadriid. (Rostratulin.).

Rostrhamnus LESSON 1830: Av. FALCONIF, Falconid. (Milvin.). 
Rostrogobio : P.-CYPRINIF. (Cyprinoid.) Cyprinid.

Rothonia : s./g. de Diomedea LINNE 1758.

Rotschildia MOCQUARD 1905 : syn. de Mocquardia AHL 1931.

Rougetius BONAPARTE 1856: Av. RALLIF. Rallid.

Roughleyia WHITLEY 1931 : P.PERCIF. (Percoid.) Sparid.

Rouleina JORDAN 1923 : syn. de Jordanites FOWLER 1925.

Rouleina FOWLER 1925: syn. d'Eurypharynx VAILLANT 1882.

Rouleophis CHABANAUD 1916 : R.-SQUAMAT. (Serpent.) Boigid.

Roulini CUVIER 1829 : s./g. de Tapirus LINNE 1758.

Rousettus GRAY 1821: M.-CHIROPT. (Megachiropt.) Pteropid. (Pteropin.).

Rovettia LOWE 1923 : Av. PASSERIF. (Acromyod.) Fringillid.

Roxasella FOWLER 1943 : P.-PERCIF. (Trachinoid.) Trachinid.

Royigerygone MATHEWS 1912 : syn. de Gerygone GOULD 1841.

Rubellus FITZINGER 1874 : P.-CYPRINIF. (Cyprinoid.) Cyprinid.

Rubigula BLYTH 1845: Av. PASSERIF. (Acromyod.) Pycnonotid. ou Parid.

Ruboralga WHITLEY 1931: P.PERCIF. (Scorpaenoid.) Scorpaenid.

Rubrisciurus ELLERMAN 1954 : s./g. de Callosciurus GRAY 1867.

Rucervus HODGSON 1838 : M.-PARAXON. Cervid.

Rudarius JORDAN et F OWLER 1902: P.-PERCIF. (Balistoid.) Balistid. ou Monacanthid.

Rudolphius GRAY 1866: syn. de Balaenoptera de LACEPEDE 1804.

Rukaia GRAY 1867 : syn. de Ratufa GRAY 1867.

Rukia MOMIYAMA 1922 : syn. de Zosterops VIGORS et HORSFIELD 1826.

Runcinatus WHITLEY 1929 : P.SYNGNATHIF. Syngnathid.
Runula JORDAN et BOLLMAN 1889 : P.-PERCIF. (Blennioid.) Blenniid.

Rupestes THOMAS $1922: \mathrm{s} . / \mathrm{g}$. de Sciurotamias MILLER 1901.

Rupicapra de BLAINVILLE 1816 : M.-PARAXON. Bovid. (Rupicaprin.).

Rupicapra FRISCH 1777 : nom. nud. pro Rupicapra de BLAINVILLE 1816.

Rupicola BRISSON 1760 : Av. PASSERIF. (Mesomyod.) Cotingid.

Rupiscartes SWAINSON 1839 : P. PERCIF. (Blennioid.) Blenniid.

Rupornis KAUP 1845 : Av. FALCONIF. Falconid.

Ruppelia SWAINSON 1839: P.PERCIF. (Gobioid.) Gobiid.

Ruppellia CASTELNAU 1873: P.PERCIF. (Percoid.) Plesiopid.

Rusa SMITH 1827: M.-PARAXON. Cervid.

Ruscariops HUBBS 1926 : P.-PERCIF. (Cottoid.) Cottid.

Ruscarius JORDAN et STARKS 1895 : P.-PERCIF. (Cottoid.) Cottid.

Rusciculus GREELY 1901 : P.-PERCIF. (Cottoid.) Cottid.

Rusulus STARKS et MANN 1911: P.-PERCIF. (Cottoid.) Cottid.

Ruticilla BREHM 1828: syn. de Phoenicurus FORSTER 1817.

Rutilus RAFINESQUE 1820 : P. CYPRINIF. (Cyprinoid.) Cyprinid.

Ruvettus COCCO 1833 : P.-PERCIF. (Trichiuroid.) Gempylid.

Ruvenzorornis NEUMANN 1903 : Av. CUCULLIF. Musophagid.

Rypticus CUVIER et VALENCIENNES 1829: P.-PERCIF. (Percoid.) Serranid.

Ryzaena ILLIGER 1811: syn. de Suricata DESMAREST 1804.

\section{$\mathbf{s}$}

Sabanejewia VLADYKOV 1928 : P.CYPRINIF. (Cyprinoid.) Cobitid.

Sabota ROBERTS 1922: Av. PASSERIF. (Acromyod.) Alaudid. 
Sacalius SMITH 1839 : syn. de Canis LINNE 1758 .

Saccarius GUNTHER 1861 : P.-LOPHIIF. (Antennarioid.) Antennariid.

Saccobranchus CUVIER et VALENCIENNES 1840 : P.-CYPRINIF, (Siluroid.) Clariid.

Saccodeira GIRARD 1857 : syn. de Proctotretus DUMERIL et BIBRON 1837.

Saccoderma SCHULTZ 1944: P.CYPRINIF. (Characoid.) Characid.

Saccodon KNER et STEINDACHNER 1863 : P.-CYPRINIF, (Characoid.) Characid.

Saccogaster A L C O C K 1889 : P.PERCIF. (Ophidioid.) Brotulid.

Saccolaimus LESSON 1842: $s . / g$. de Taphozous OKEN 1816.

Saccolaimus GRAY 1866 : syn. de Taphozous OKEN 1816.

Saccomys CUVIER 1823 : syn. d'Heteromys DESMAREST 1817.

Saccopharynx MITCHILL 1824 : P.SACCOPHARYNGIF. Saccopharyngid.

Saccopteryx ILLIGER 1811 : M.CHIROPT. (Microchiropt.) Emballonurid.

Saccostoma SAUVAGE 1882: syn. de Chiasmichthys JORDAN 1903.

Saccostomus PETERS 1846 : M.RODENT. (Myomorph.) Murid. (Murin.).

Sacrestinus JORDAN, EVERMANN et TANAKA 1927: P.-PERCIF. (Percoid.) Lutjanid.

Sacura JORDAN et RICHARDSON 1910 : P.-PERCIF. (Percoid.) Serranid.

Saepiopterus REICHENBACH 1854: Av, APODIF. Trochilid.

Safole JORDAN 1912 : syn. de Moronopsis GILL 1863.

Sagamia JORDAN et SNYDER 1901: P.-PERCIF. (Gobioid.) Gobiid.

Sagamichthys : P.-CLUPEIF. (Elopoid.) Elopid.

Sagenichthys BERG 1895 : P.-PERCIF. (Percoid.) Sciaenid.
Sagittabarilius FOWLER 1936 : s./g. de Barilius HAMILTON 1822.

Sagittarius HERMANN 1783: Av. FALCONIF, Sagittariid.

Saiga GRAY 1843: M.-PARAXON. Bovid. (Caprin.).

Saimiri VOIGT 1831: M.-PRIMAT. (Simioid.) Cebid. (Cebin.).

Sakesphorus : Av. PASSERIF. (Mesomyod.) Formicariid.

Salamandra GRONOV 1763 : Amph. UROD. Salamandrid.

Salamandrella 'DYBOWSKI 1870 : Amph. UROD. Ambyostomatid.

Salamandrina 'FITZINGER '1826 : Amph. UROD. Salamandrid.

Salangana STREUBEL 1848 : syn. de Collocalia GRAY 1840.

Salanx OKEN 1817 : P.-CLUPEIF (Salmonoid.) Salangid.

Salar CUVIER et VALENCIENNES 1848: syn. de Salmo LINNE 1758.

Salarias CUVIER 1817 : P.-PERCIF. (Blennioid.) Blenniid.

Salarichthys GUICHENOT 1867 : P.-PERCIF. (Blennioid.) Blenniid.

Salea GRAY 1845: R.-SQUAMAT. (Saur.) Agamid.

Salema JORDAN et EVERMANN 1896 : P.-PERCIF. (Percoid.) Sparid.

Salilota GUNTHER 1887: P.-GADIF. Gadid.

Salmacis GLOGER 1841: syn. de Macaca de LACEPEDE 1799.

Salminus AGASSIZ 1829: P.-CYPRINIF. (Characoid.) Characid.

Salmo LINNE 1758 : P.-CLUPEIF. (Salmonoid.) Salmonid.

Salmoperca THOMPSON 1850 : $P$. PERCOPSIF. Percopsid.

Salmophasia SWAINSON 1839 : P.CYPRINIF. (Cyprinoid.) Cyprinid.

Salmostoma SWAINSON 1839 : P.CYPRINIF. (Cyprinoid.) Cyprinid.

Salmothymus BERG 1907 : P.-CLUPEIF. (Salmonoid.) Salmonid. 
Salpinctes CABANIS 1847: Av. PASSERIF. (Acromyod.) Troglodytid.

Salpingotus VINOGRADOV 1923 : M.-RODENT. (Myomorph.) Dipodid. (Cardiocranin.).

Salpornis GRAY 1847 : Av. PASSERIF. (Acromyod.) Certhiid.

Saltator VIEILLOT 1816 : Av. PASSERIF. (Acromyod.) Fringillid. (Pitylin.).

Salvadora BAIRD et GIRARD 1853: R.-SQUAMAT. (Serpent.) Colubrid.

Salvadorina ROTSCHILD et HARTERT 1894: Av. ANATIF. Anatid. (Anatin.).

Salvator DUMERIL et BIBRON 1839: syn. de Tupinambis DAUDIN 1802.

Salvelinus RICHARDSON 1836 : P.CLUPEIF. (Salmonoid.) Salmonid.

Samaris GRAY 1831 : P.-PLEURONECTIF. (Soleoid.) Cynoglossid.

Samariscus GILBERT 1905: P.PLEURONECTIF. (Soleo $i d$.) Cynoglossid.

Sambur HEUDE 1888 : syn. de Cervus LINNE 1758.

Sambwa: vide Sawbwa ANNANDALE 1918.

Samuela MATHEWS 1912 : syn. de Cinclosoma VIGORS et HORSFIELD 1826.

Sanagia HOLLY 1926 : P.-CYPRINIF. (Cyprinoid.) Cyprinid.

Sandakanus HERRE $1942: s . / g$. de Neostethus REGAN 1916.

Sandat CLOQUET 1827: P.-PERCIF. (Percoid.) Percid.

Sandelia CASTELNAU 1861: P.PERCIF. (Anabantoid.) Anabantid.

Sander OKEN 1817 : P.-PERCIF. (Percoid.) Percid.

Sanfordia McMURPHY et MATHEWS 1929: Av. PASSERIF. (Acromyod.) Zosteropid.

Sanopus SMITH 1952 : P.-PERCIF. (Batrachoid.) Batrachoidid.

Sanzinia GRAY 1849: R.-SQUAMAT. (Serpent.) Boid.
Sapheopipo HARGITT 1890: Av. PICIF. Picid. (Picin.).

Sappho REICHENBACH 1849: Av. APODIF. Trochilid.

Saraca STEINDACHNER 1875 : P.PERCIF. (Percoid.) Cichlid.

Sarchirus RAFINESQUE 1818 : P.AMIIF. Lepisosteid.

Sarcidiornis AGASSIZ 1846 : emend. pro Sarkidiornis EYTON 1838.

Sarcidium COPE 1872 : P.-CYPRINIF. (Cyprinoid.) Cyprinid.

Sarcina RAFINESQUE 1810: P.PERCIF. (Trichiuroid.) Trichiurid.

Sarciophorus STRICKLAND 1841: Av. CHARADRIIF. Charadriid. (Charadriin.).

Sarcocheilichthys BLEEKER 1860 : P.-CYPRINIF. (Cyprinoid.) Cyprinid.

Sarcodaces GUNTHER 1864: P.CYPRINIF. (Characoid.) Characid.

Sarcogeranus SHARPE 1893: Av. RALLIF. Gruid.

Sarcogrammus REICHENBACH 1852: Av. CHARADRIIF. Charadriid. (Charadriin.).

Sarcophanops SHARPE 1877 : Av. PASSERIF. (Desmodactyl.) Eurylaimid.

Sarcophilus CUVIER 1837: M.MARSUP. Dasyurid. (Dasyurin.).

Sarcops WALDER 1875: Av. PASSERIF. (Acromyod.) Sturnid.

Sarcorhamphus DUMERIL 1806 : Av. FALCONIF. Vulturid.

Sarda de LACEPEDE 1802: P. PERCIF. (Scombroid.) Scombrid.

Sardina ANTIPA 1905: P.-CLUPEIF. (Clupeoid.) Clupeid.

Sardinella CUVIER et VALENCIENNES 1847: P.-CLUPEIF. (Clupeoid.) Clupeid.

Sardinia POEY 1860 : P.-CLUPEIF. (Clupeoid.) Clupeid.

Sardinops HUBBS 1929: P.-CLUPEIF. (Clupeoid.) Clupeid.

Sargocentron FOWLER 1904 : P.BERYCIF. Holocentrid. 
Sargochromis REGAN 1920: P.PERCIF. (Percoid.) Cichlid.

Sargosomus AGASSIZ 1861: P.PERCIF, (Labroid.) Embiotocid.

Sargus WALBAUM 1792: P.-PERCIF. (Percoid.) Sparid.

Sarkidiornis EYTON 1838: Av. ANATIF, Anatid. (Anserin.).

Saroglossa BLYTH 1844: Av. PASSERIF. (Acromyod.) Sturnid. (Sturnin.).

Sarotherodon RUPPELL 1852 : P.PERCIF. (Percoid.) Cichlid.

Sarothrodus GILL 1861: P.-PERCIF. (Percoid.) Chaetodontid.

Sarothrura HEINE 1888: Av. RALLIF. Rallid.

Sarpa BONAPARTE 1831 : P.-PERCIF. (Percoid.) Sparid.

Sarritor JORDAN et EVERMANN 1896 : P.-PERCIF. (Cottoid.) Agonid.

Sartor MYERS et de CARVALHO 1959: P.-CYPRINIF. (Characoid.) Characid.

Sasia HODGSON 1836: Av. PICIF. Picid. (Picumnin.).

Sassius ROTSCHILD et HARTERT 1926: Av. PASSERIF. (Acromyod.) Drepanidid.

Satan HUBBS 1947 : P.-CLYPEIF. (Siluroid.) Bagrid.

Satanellus POCOCK 1926 : M.-MARSUP. Dasyurid. (Dasyurin.).

Satanoperca GUNTHER 1862: P.PERCIF. (Percoid.) Cichlid.

Sathrocerus NEUMANN 1920: Av. PASSERIF. (Acromyod.) Sylviid.

Sator : R.-SQUAMAT. (Saur.) Iguanid.

Satrapa STRICKLAND 1844: Av. PASSERIF. (Mesomyod.) Tyrannid. (Fluvicolin.).

Satsuma SMITH et POPE 1906 : P.PERCIF. (Percoid.) Serranid.

Satyrus OKEN 1816 : syn. de Pongo de LACEPEDE 1799.

Saucerottia BONAPARTE 1849 : Av. APODIF. Trochilid.

Saundersia DWIGHT 1925 : syn. de Saundersilarus DWIGHT 1926.

Saundersilarus DWIGHT 1926 : Av. LARIF. Larid. (Larin.).
Saurenchelys PETERS 1864: P. ANGUILLIF. (Congroid.) Nettastomid.

Sauresia GRA Y 1852 : R.-SQUAMAT. (Saur.) Anguid.

Saurichthys AGASSIZ 1834 : P.-

Saurichthyid.

Saurida CUVIER et VALENCIENNES 1849 : P.-CLUPEIF. (Myctophoid.) Synodid.

Sauridichthys BLEEKER 1858 : P.CLUPEIF. (Myctophoid.) Myctophiid.

Saurogobio BLEEKER 1870 : P.CYPPRINIF. (Cyprinoid.) Cyprinid.

Sauromalus DUMERIL 1856 : R.SQUAMAT. (Saur.) Iguanid.

Sauromarptis CABANIS et HEINE 1860 : Av. CORACIADIF. Alcedinid.

Sauromuraenesox ALCOCK 1889 : P.-ANGUILLIF. (Congroid.) Congrid.

Sauropatis CABANIS et HEINE 1860 : syn. de Halcyon SWAINSON 1821.

Sauropatoides LAUBMANN 1926 : s./g. d'Alcedo LINNE 1758.

Saurophis FITZINGER $1826:$ s./g. de Tetradactylus MERREM 1820 .

Saurothera VIEILLOT 1816: Av. CUCULLIF. Cucullid.

Saurus CUVIER 1817: P.-CYPRINIF. (Siluroid.) Bagrid.

Saurus S.D.W. 1837: syn. de Synodus GRONOV 1763.

Sauvagella BERTIN 1940 : P.-CLUPEIF. (Clupeoid.) Dussumierid.

Sawara JORDAN et HUBBS 1925 : P. - PERCIF. (Scombroid.) Scombrid.

Sawbwa ANNANDALE 1918: P.CYPRINIF. (Cyprinoid.) Cyprinid.

Saxicola BECHSTEIN 1802: Av. PASSERIF. (Acromyod.) Turdid. (Saxicolin.).

Saxicoloides GRAY 1841 : Av. PASSERIF. (Acromyod.) Turdid. (Saxicolin.).

Saxilaga SCOTT 1935 : P.-CLUPEIF. (Galaxioid.) Galaxiid.

Saxilauda LESSON 1837 : Av. PASSERIF. (Acromyod.) Alaudid. 
Sayonara JORDAN et SEALE 1906: P.-PERCIF. (Percoid.) Serranid.

Sayornis BONAPARTE 1854: Av. PASSERIF. (Mesomyod.) Tyrannid. (Fluvicolin.).

Sayris RAFINESQUE 1810 : P.-BELONIF. (Belonid.) Scombresocid.

Scaeophaëton MATHEWS 1913 : Av. PELECANIF. Phaetontid.

Scaeops JORDAN et STARKS 1904 : P. - PLEURONECTIF. (Pleuronectoid.) Bothid.

Scaeopus PETERS 1861: M.EDENT. (Xenarthr.) Bradypodid.

Scaevius : P.-PERCIF. (Percoid.) Lutjanid.

Scalabotes PETERS 1880: R.SQUAMAT. (Saur.) Geckonid.

Scalanago WHITLEY 1935 : P.-ANGUILLIF. (Congroid.) Congrid.

Scalicus JORDAN 1923: P.-PERCIF. (Scorpaenoid.) Triglid.

Scalops CUVIER 1800: syn. de Scalopus GEOFFROY - ST - HILAIRE 1803.

Scalopus GEOFFROY-ST-HILAIRE 1803 : M.-INSECTIV. Talpid. (Scalopin.).

Scapaneus CABANIS et HEINE 1863: Av. PICIF. Picid. (Picin.),

Scapanulus THOMAS 1912: M.-INSECTIV. Talpid. (Scalopin.).

Scapanus POMEL 1848 : M.-INSECTIV. Talpid. (Scalopin.).

Scaphesthes OSHIMA 1919 : P.-CYPRINIF. (Cyprinoid.) Cyprinid.

Scaphiodon HECKEL 1843 : P.-CYPRINIF. (Cyprinoid.) Cyprinid.

Scaphiodonichthys VINCIGUERRA 1890 : P.-CYPRINIF. (Cyprinoid.) Cyprinid.

Scaphiodontella OSHIMA 1920 : P.CYPRINIF. (Cyprinoid.) Cyprinid.

Scaphiodontophis TAYLOR et SMITH 1943 : R.-SQUAMAT. (Serpent.) Colubrid.

Scaphiodontopsis FOWLER 1934 : syn. de Scaphiodonichthys VINCIGUERRA 1890.
Scaphiophis PETERS $1870:$ R.SQUAMAT. (Serpent.) Colubrid.

Scaphiophryne BOULENGER 1882: Amph. ANOUR. Microhylid.

Scaphiopus HOLBROOK 1835 : Amph. ANOUR. Pelobatid.

Scaphirhynchops GILL 1863 : P.ACIPENSERIF. Acipenserid.

Scaphirhynchus HECKEL 1835 : P.ACIPENSERIF. Acipenserid.

Scaphognathops SMITH 1945 : P.PERCIF. (Gobioid.) Gobiid.

Scaphognathus SMITH 1931: syn. de Scaphognathops SMITH 1945.

Scapirhynchus : vide Scaphirhynchus HECKEL 1835.

Scapteira WIEGMANN 1834: R.SQUAMAT. (Saur.) Lacertid.

Scapteromys WATERHOUSE 1837: M.-RODENT. (Myomorph.) Cricetid. (Hesperomyin.).

Scaptira AGASSIZ 1846: emend. pro Scapteira WIEGMANN 1834.

Scaptochirus MILNE-EDWARDS 1867: syn. de Talpa LINNE 1758.

Scaptonyx MILNE-EDWARDS 1872: M.-INSECTIV. Talpid. (Scalopin.).

Scardafella BONAPARTE 1854: Av. COLUMBIF. Columbid. (Columbin.).

Scardiniopsis JACKEL 1864: P.CYPRINIF. (Cyprinoid.) Cyprinid.

Scardinius BONAPARTE 1840 : P.CYPRINIF. (Cyprinoid.) Cyprinid.

Scardinopsis BENECKE 1884 : P.CYPRINIF. (Cyprinoid.) Cyprinid.

Scarichthys BLEEKER 1861: syn. de Leptoscarus $S$ W A I N S ON 1839.

Scaridea JENKINS 1904: P.-PERCIF. (Labroid.) Scarid.

Scarodon TEMMINCK et SCHLEGEL 1845 : P.-CYPRINIF. (Characoid.) Characid.

Scaroides TOULA 1909: P.-PERCIF. (Labroid.) Scarid. 
Scarops SCHULTZ 1958: P.-PERCIF. (Labroid.) Scarid.

Scarostoma KNER 1867: P.-CYPRINIF. (Characoid.) Characid.

Scartelaos SWAINSON 1839: P.PERCIF. (Gobioid.) Periophthalmid.

Scartella JORDAN 1886 : P.-PERCIF. (Blennioid.) Blenniid.

Scartes JORDAN et EVERMANN 1896: syn. de Scartichthys JORDAN et EVERMANN 1898.

Scartichthys JORDAN et EVERMANN 1898 : P.-PERCIF. (Blennioid.) Blenniid.

Scartiscus COPE 1862: R.-SQUAMAT. (Sanr.) Iguanid.

Scartoblennius FOWLER 1946 : P.PERCIF. (Blennioid.) Blenniid.

Scartomyzon FOWLER $1913: s . / g$. de Moxostoma RAFINESQUE 1820 .

Scarturus GOLGER 1841: syn. d'Allactaga CUVIER 1836.

Scarus GRONOV 1763 : syn. de Callyodon GRONOV 1763.

Scarus FORSKAL 1775 : P.-PERCIF. (Labroid.) Scarid.

Scatharus CUVIER et VALENCIENNES 1830: P. - PERCIF. (Percoid.) Sparid.

Scatophagus CUVIER et VALENCIENNES 1831: P. - PERCIF. (Percoid.) Chaetodontid.

Sceloglaux KAUP 1848: Av. STRIGIF. Strigid. (Bubonin.).

Scelonorus WIEGMANN 1828: R.SQUAMAT. (Saur.) Iguanid.

Scelorchilus OBERHOLSER 1923 : Av. PASSERIF. (Mesomyod.) Pteroptochid.

Scelotes FITZINGER 1826: R.SQUAMAT. (Saur.) Scincid.

Scenopaeetes COUES 1891: Av. PASSERIF. (Acromyod.) Paradiseid. (Paradisein.).

Scenopoeus RAMSAY 1876: Av. PASSERIF. (Acromyod.) Paradiseid. (Ptylonorhynchin.).

Scepomycter GRANT et MACKWORTH - PREAD 1941: Av. PASSERIF. (Acromyod.) Nectariniid.

Scepterias JORDAN 1922 : P.-PERCIF. (Percoid.) Serranid.
Schacra GUNTHER 1868: P.-CYPRINIF. (Cyprinoid.) Cyprinid.

Schaeffia HILZHEIMER 1906 : syn. de Canis LINNE 1758.

Schaenicola BONAPARTE 1850 : Av. PASSERIF. (Acromyod.) Sylviid.

Schedophilopsis STEINDACHNER 1881 : P.-PERCIF. (Icosteoid.) Icosteid.

Schedophilus COCCO 1829: P.PERCIF. (Icosteoid.) Icosteid.

Schebta LESSON 1831 : Av. PASSERIF. (Acromyod.) Vangid.

Schiffornis BONAPARTE 1854: Av. PASSERIF. (Mesomyod.) Piprid.

Schilbe OKEN 1817 : P.-CYPRINIF. (Siluroid.) Schilbeid.

Schilbeichthys BLEEKER 1858 : P.CYPRINIF. (Siluroid.) Schilbeid.

Schilbeodes BLEEKER 1858: P.CYPRINIF. (Siluroid.) Schilbeid.

Schilus JAROCKI 1822 : P.-PERCIF. (Percoid.) Percid.

Schindleria GILTAY 1934 : P.-PERCIF. (Ophidioid.) Fierasferid.

Schipa BRANDT 1869 : P.-ACIPENSERIF. Acipenserid.

Schismatogobius BEAUFORT 1912 : P.-PERCIF. (Gobioid.) Gobiid.

Schismatorhynchos BLEEKER 1860 : P.-CYPRINIF. (Cyprinoid.) Cyprinid.

Schistes GOULD 1851: Av. APODIF. Trochilid.

Schistochlamys REICHENBACH 1850: Av. PASSERIF. (Acromyod.) Tanagrid.

Schistocichla TODD 1927: Av. PASSERIF. (Mesomyod.) Formicariid.

Schistometopon PARKER 1941: Amph. APOD. Caeciliid.

Schistoperca FOWLER 1943: P.PERCIF. (Percoid.) Serranid.

Schistorus GILL 1863: s./g. d'Epinephelus BLOCH 1793.

Schistospiza SHARPE 1888 : syn. de Lophospingus CABANIS 1878.

Schizocypris REGAN 1914 : P.CYPRINIF. (Cyprinoid.) Cyprinid. 
Schizodon WATERHOUSE 1841 : syn. d'Aconaemys AMEHINGO 1891.

Schizodon AGASSIZ 1829 : P.-CYPRINIF. (Characoid.) Characid.

Schizodontopsis GARMAN 1890 : P.-CYPRINIF. (Characoid.) Characid.

Schizoeaca CABANIS 1873: Av. PASSERIF. (Mesomyod.) Furnariid.

Schizophallus HUBBS $1926: s . / g$. de Gambusia POEY 1854.

Schizopygopsis STEINDACHNER 1866: P. - CYPRINIF. (Cyprinoid.) Cyprinid.

Schizonyge HECKEL 1847 : P.-CYPRINIF. (Cyprinoid.) Cyprinid.

Schizorhis WAGLER 1829: Av. CUCULLIF. Musophagid.

Schizorhiza WEILER 1930 : P.RAJIF. (Rhinobatoid.) Pristid.

Schizostoma GERVAIS 1855: syn. de Glyphonycteris THOS 1896.

Schizothorax HECKEL 1838: P.CYPRINIF. (Cyprinoid.) Cyprinid.

Schizura CABANIS 1847 : Av. PASSERIF. (Mesomyod.) Furnariid.

Schlegelia BERNSTEIN 1861: Av. PASSERIF. (Acromyod.) Paradiseid. (Paradisein.).

Schmidtia JORDAN et STARKS 1904: syn. de Schmidtina JORDAN et STARKS 1904.

Schmidtina JORDAN et STARKS 1904 : syn. de Stlengis JORDAN et STARKS 1904.

Schmidtophis TAYLOR 1942: R.SQUAMAT. (Serpent.) Colubrid.

Schoeinophylax RIDGWAY 1909: Av. PASSERIF. (Mesomyod.) Furnariid.

Schoenicola BLYTH 1844: Av. PASSERIF. (Acromyod.) Sylviid.

Schoeniparus DELACOUR et JABOUILLE 1928: Av. PASSERIF. (Acromyod.) Timaliid.

Schoinobates LESSON 1842: M.MARSUP. Phalangerid. (Phascolarctin.).

Schoutedenella de WITTE 1921: Amph. ANOUR. Brachycephalid.
Schubotzia BOULENGER 1914 : P.PERCIF. (Percoid.) Cichlid.

Schuettea STEINDACHNER 1866 : P.-PERCIF. (Percoid.) Monodactylid.

Schultzea WOODS 1958: P.-PERCIF. (Percoid.) Dipterygonotid.

Schulzidia GOSLINE 1952 : s./g. de Muraenichthys BLEEKER 1853.

Schwetzochromis : P. - PERCIF. (Percoid.) Cichlid.

Sciadeichthys BLEEKER 1863 : P.CYPRINIF. (Siluroid.) Bagrid.

Sciadeoides EINGENMANN 1888: P.-CYPRINIF. (Siluroid.) Bagrid.

Sciades MULLER et TROSCHEL 1849 : P.-CYPRINIF. (Siluroid.) Bagrid.

Sciaena LINNE 1758 : P.-PERCIF. (Percoid.) Sciaenid.

Sciaenoides RICHARDSON 1850 : P.-PERCIF. (Percoid.) Sciaenid. ou Cheilodactylid.

Sciaenops GILL 1863 : P.-PERCIF. (Percoid.) Sciaenid.

Scianectes ALCOCK 1889 : syn. de Laeops GUNTHER 1880.

Scidorhombus TANAKA 1915 : P.PLEURONECTIF. (Pleuronectoid.) Bothid.

Sciliogaleus BOULENGER 1902 : errat. pro Scylliogaleus $\mathrm{BOU}$ LENGER 1902.

Scincella MITTELMAN $1950:$ R.SQUAMAT. (Saur.) Scincid.

Scincus GRONOV 1763: R.-SQUAMAT. (Saur.) Scincid.

Scirtetes WAGNER 1841: syn. d'Allactaga CUVIER 1836.

Scirtomys BRANDT 1844: syn. d'Allactaga CUVIER 1836.

Scirtopoda POCOCK 1922 : syn. de Jaculus ERXLEBEN 1777.

Scirtopoda BRANDT 1844: M.-RODENT. (Myomorph.) Dipodid. (Dipodin.).

Scissirostrum de LAFRESNAYE 1845: Av. PASSERIF. (Acromyod.) Sturnid. (Graculin.).

Scissor GUNTHER 1864 : P.-CYPRINIF. (Characoid.) Characid. 
Sciurillus THOMAS 1914: M.-RODENT. (Sciuromorph.) Sciurid. (Sciurin.).

Sciuropterus CUVIER 1825: syn. de Petromys CUVIER 1800.

Sciurotamias MILLER 1901: M.RODENT. (Sciuromorph.) Sciurid. (Sciurin.).

Sciurus LINNE 1758 : M.-RODENT. (Sciuromorph.) Sciurid. (Sciurin.).

Sclateria OBERHOLSER 1899: Av. PASSERIF. (Mesomyod.) Formicariid.

Sclerocottus FISCHER 1885: P.PERCIF. (Trachinoid.) Trachinid.

Sclerognathus CUVIER et VALENCIENNES 1844 : P.-CYPRINIF. (Cyprinoid.) Catostomid.

Scleromystax GUNTHER 1864 : P.CYPRINIF. (Siluroid.) Callichthyid.

Scleronema EIGENMANN 1918: P.-CYPRINIF. (Siluroid.) Trichomycterid.

Scleropages GUNTHER 1864: P.CLUPEIF. (Osteoglossoid.) Osteoglossid.

Scleroporus : vide Sceloporus WIEGMANN 1828.

Scleroptila BLYTH 1852 : Av. PHASIANIF. Phasianid.

Sclerotis HUBBS 1926 : P.-PERCIF, (Percoid.) Centrarchid.

Sclerurus SWAINSON 1827: Av. PASSERIF. (Mesomyod.) Furnariid.

Scobinichthys WHITLEY 1931 : P.PERCIF. (Balistoid.) Balistid.

Scolecomorphus BOULENGER 1883: Amph. APODE. Caeciliid.

Scolecophis FITZINGER 1843 : R.SQUAMAT. (Serpent.) Boigid.

Scolecosaurus BOULENGER 1885 : R.-SQUAMAT, (Saur.) Tejid.

Scolecoseps LOVERIDGE 1920 : R.SQUAMAT. (Saur.) Scincid.

Scolecosoma GIRARD 1858: $\mathrm{Cy}$ clost. PETROMYZONIF. Petromyzonid.

Scolenchelys OGILBY 1897 : P.-ANGUILLIF. (Congroid.) Congrid. ou Echelid.
Scoliodon MULLER et HENLE 1837: P.-GALEIF. (Carcharhinoid.) Carcharhinid.

Scoliostomus RUPPELL 1828: P. CLUPEIF. (Chanoid.) Chanid.

Scolomys ANTHONY 1924 : M.-RODENT. (Myomorph.) Cricetid. (Cricetin.).

Scolopacichthys REGAN 1914 : P.AULOSTOMIF. Macrorhamphosid.

Scolopax LINNE 1758 : Av. CHARADRIIF. Charadriid. (Scolopacin.).

Scolopsides CUVIER 1829 : P.-PERCIF. (Percoid.) Lutjanid.

Scolopsis CUVIER 1814: P.-PERCIF. (Percoid.) Lutjanid.

Scomber LINNE 1758 : P.-PERCIF, (Scombroid.) Scombrid.

Scomberesox : vide Scombresox de LACEPEDE 1803.

Scomberoides de LACEPEDE 1802: P.-PERCIF. (Scombroid.) Scombrid.

Scomberomorus de LACEPEDE 1802 : P.-PERCIF. (Scombroid.) Scombrid.

Scombresox de LACEPEDE 1803 : P.-BELONIF. (Belonoid.) Scombresocid.

Scombrocottus PETERS 1872 : P.PERCIF. (Scorpaenoid.) Hexagrammid.

Scombrocypris GUNTHER 1889 : P.-CYPRINIF. (Cyprinoid.) Cyprinid.

Scombrolabrax ROULE 1921 : P.PERCIF. (Percoid.) Serranid.

Scombrops TEMMINCK et SCHLEGEL 1845: P.-PERCIF. (Percoid.) Serranid.

Scopelarchoides PARR 1929: P.CLUPEIF. (Alepisauroid.) Scopelarchid.

Scopelarchus ALCOCK 1896: P.CLUPEIF. (Alepisauroid.) Scopelarchid.

Scopelengys A L C OCK 1890 : P.CLUPEIF. (Myctophoid.) Myctophiid.

Scopeloberyx ZUGMAYER 1911 : P. - STEPHANOBERYCIF. Melamphaeid. 
Scopelogadus VAILLANT 1888 : P.-STEPHANOBERYCIF, Melamphaeid.

Scopelopsis BR A U E 1906 : P.CLUPEIF. (Myctophoid.) Myctophiid.

Scopelosaurus BLEEKER 1860 : P. -CLUPEIF. (Myctophoid.) Aulopid.

Scopelus CUVIER 1817: syn. de Myctophum RAFINESQUE 1810 .

Scophthalmus RAFINESQUE 1810 : P. - PLEURONECTIF. (Pleuronectoid.) Bothid.

Scops SAVIGNY 1809 : syn. de Otus PENNANT 1769.

Scoptelus CABANIS et HEINE 1860: Av. CORACIADIF. Upupid. (Phoeniculin.).

Scopus BRISSON 1760: Av. ARDEIF. Scopid.

Scorpaena LINNE 1758: P.-PERCIF. (Scorpaenoid.) Scorpaenid.

Scorpaenella FOWLER 1938 : P.PERCIF. (Scorpaenoid.) Scorpaenid.

Scorpaenichthys GIRARD 1854 : P.-PERCIF. (Cottoid.) Cottid.

Scorpaenodes BLEEKER 1857 : P.PERCIF. (Scorpaenoid.) Scorpaenid.

Scorpaenopsella FOWLER 1938: P.-PERCIF. (Scorpaenoid.) Scorpaenid.

Scorpaenopsis HECKEL 1839 : P.PERCIF. (Scorpaenoid.) Scorpaenid.

Scorpiodoras EIGENMANN 1925: P.-CYPRINIF. (Siluroid.) Doradid.

Scorpis CUVIER et VALENCIENNES 1831: P.-PERCIF. (Percoid.) Chaetodontid.

Scortornis : vide Scotornis SWAINSON 1837.

Scortum WHITLEY 1943 : P.-PERCIF. (Percoid.) Serranid.

Scoteinus DOBSON $1875: \mathrm{s} . / \mathrm{g}$. de Nycticeius RAFINESQUE 1819.

Scotiaptex SWAINSON 1836: Av. STRIGIF. Strigid. (Strigin.).

Scotinomys THOMAS 1913 : M.-RO-
DENT. (Myomorph.) Cricetid. (Hesperomyin.).

Scotobleps BOULENGER 1900 : Amph. ANOUR. Ranid.

Scotocerca SUNDEVALL 1872 : Av. PASSERIF. (Acromyod.) Sylviid. ou Timaliid.

Scotocichla SHARPE 1883: Av. PASSERIF. (Acromyod.) Timaliid.

Scotoecus THOMAS 1901 : M.-CHIRUPT. (Microchiropt.) Vespertilionid. (Vespertilionin.).

Scotomanes DOBSON 1875: M.CHIROPT. (Microchiropt.) Vespertilionid. (Vespertilionin.).

Scotonycteris MATSCHIE 1894 : M. - CHIROPT. (Megachiropt.) Pteropid. (Pteropin.).

Scotopelia BONAPARTE 1849 : Av. STRIGIF. Strigid. (Bubonin.).

Scotophilus LEACH 1821 : M.-CHIKOPT. (Microchiropt.) Vespertilionid. (Vespertilionin.).

Scotophis BAIRD et GIRARD 1853 : R.SQUAMAT. (Serpent.) Colubrid.

Scotornis SWAINSON 1837: Av. CAPRIMULGIF. Caprimulgid. (Caprimulgin.).

Scotozous DOBSON 1875 : syn. de Pipistrellus KAUP 1829.

Scrofa GRAY 1868: syn. de Sus LINNE 1758.

Scrofaria GISTEL 1848: P.-PERCIF. (Luvaroid.) Luvarid.

Scrophicephalus SWAINSON 1838 : P.-CLUPEIF. (Mormyroid.) Mormyrid.

Scurrilichthys FRASER-BRUNNER $1941: s . / g$. d'Arotrolepis.

Scutengraulis JORDAN et SEALE 1925: P.-CLUPEIF. (Clupeoid.) Engraulid. ou Clupeid.

Scutia JORDAN et EVERMANN 1896: syn. d'Uropterygius RUPPELL 1838.

Scuticaria JORDAN et SNYDER 1901: P.-ANGUILLIF. (Anguilioid.) Muraenid.

Scutiger THEOBALD 1868 : Amph. ANOUR. Pelobatid.

Scutisorex THOMAS 1913: M.-INSECTIV. Soricid. (Scutisoricin.). 
Scyliogaleus : vide Scylliogaleus BOULENGER 1902.

Scyliorhinus de BLAINVILLE 1816 P. - GALEIF. (Carcharhinoid.) Scyliorhinid.

Scylliogaleus BOULENGER 1902 P.-GALEIF. (Carcharhinoid.) Carcharhinid.

Scylliorhinus de BLAINVILLE 1828 : emend. pro Scyliorhinus de BLAINVILLE 1816.

Scyllium CUVIER 1817: syn. de Scyliorhinus de BLAINVILLE 1816.

Scymnodalatias GARRICK 1955 : P.-SQUALIF. (Squalioid.) Squalid.

Scymnodon BOCAGE et CAPELLO 1864: P.-SQUALIF. (Squalioid.) Squalid.

Scymnorhinus BONAPARTE 1846 : P.-SQUALIF. (Squalioid.) Scymnorhinid.

Scymnus CUVIER 1817 : P.-SQUALIF. (Squalioid.) Scymnorhinid.

Scyris CÜVIER 1829 : syn. de Alectis RAFINESQUE 1815.

Scytalichthys JORDAN et DAVIS 1892 : P. - ANGUILLIF. (Congroid.) Ophichthyid.

Scytallurus DUMERIL 1856 : P.ANGUILLIF. (Congroid.) Ophichthyid.

Scytalophis KAUP 1856 : P.-ANGUILLIF. (Congroid.) Ophichthyid.

Scytalopus GOULD 1837 : Av. PAS SERIF. (Mesomyod.) Pteroptochid.

Scythrops LATHAM 1790 : Av. CUCULLIF. Cucullid.

Scytopis COPE 1862 : syn. de Phrynohyas FITZINGER 1843.

Sealeina FOWLER 1907 : P.-CYPRINIF. (Characoid.) Characid.

Searsia PARR 1937 : P.-CLUPEIF. (Alepocephaloid.) Alepocephalid.

Sebastapistes STREETS 1878: P.PERCIF. (Scorpaenoid.) Scorpaenid.

Sebastavus JORDAN et GILBERT 1919 : P.-PERCIF. (Scorpaenoid.) Scorpaenid.
Sebastella TANAKA 1918 : P.-PERCIF. (Scorpaenoid.) Scorpaenid.

Sebastes CUVIER 1829: P.-PERCIF. (Scorpaenoid.) Scorpaenid.

Sebastichthys GILL 1863 : P.-PERCIF. (Scorpaenoid.) Scorpaenid.

Sebastinus JORDAN 1920 : P.-PERCIF. (Scorpaenoid.) Scorpaenid.

Sebastiscus JORDAN et STARKS 1904 : P. - PERCIF. (Scorpaenoid.) Scorpaenid.

Sebastocarus JORDAN et EVERMANN 1927: P.-PERCIF. (Scorpaenoid.) Scorpaenid.

Sebastocles JORDAN et HUBBS 1925: P.-PERCIF. (Scorpaenoid.) Scorpaenid.

Sebastodes GILL 1861 : P.-PERCIF (Scorpaenoid.) Scorpaenid.

Sebastoessus JORDAN 1920 : P.PERCIF. (Scorpaenoid.) Scorpaenid.

Sebastolobus GILL 1881 : P.-PERCIF. (Scorpaenoid.) Scorpaenid.

Sebastomus GILL 1864: P.-PERCIF. (Scorpaenoid.) Scorpaenid.

Sebastoplus GILL 1864: P.-PERCIF. (Scorpaenoid.) Scorpaenid.

Sebastopsis GILL 1863 : P.-PERCIF. (Scorpaenoid.) Scorpaenid.

Sebastopyr JORDAN et EVERMANN 1927 : P.-PERCIF. (Scorpaenoid.) Scorpaenid.

Sebastosemus GILL 1905 : P.-PERCIF. (Scorpaenoid.) Scorpaenid.

Sectator JORDAN et FESLER 1893: P.-PERCIF. (Percoid.) Kyphosid.

Securicula GUNTHER 1868: P.CYPRINIF. (Cyprinoid.) Cyprinid.

Secutor GISTEL 1848 : P.-PERCIF. (Percoid.) Leiognathid.

Seena BLYTH 1852: Av. LARIF. Larid. (Sternin.).

Segutilum WHITLEY 1931 : P.PERCIF. (Percoid.) Kyphosid. 
Seicercus SWAINSON 1837: Av. PASSERIF. (Acromyod.) Sylviid. ou Muscicapid.

Seisura VIGORS et HORSFIELD 1826 : Av. PASSERIF. (Acromyod.) Muscicapid.

Seisurus SWAINSON 1827: Av. PASSERIF. (Acromyod.) Parulid.

Sekeetamys ELLERMAN 1947 : s./g. de Meriones ILLIGER 1811 .

Selache CUVIER 1817 : syn. de Cetorhinus de BLAINVILLE 1816.

Selanonius FLEMING 1828 : P.-GALEIF. (Isuroid.) Isurid.

Selar BLEEKER 1851 : P.-PERCIF. (Percoid.) Carangid.

Selaroides BLEEKER 1851 : P.PERCIF. (Percoid.) Carangid.

Selasphorus SWAINSON 1832: Av. APODIF. Trochilid.

Seleina BOWDICH 1825 : P.-PERCIF. (Percoid.) Kyphosid.

Selenanthias TANAKA 1918 : P.PERCIF. (Percoid.) Serranid.

Selenarctos HEUDE 1901 : M.-FISSIP. Ursid.

Selenaspis BLEEKER 1858 : P.-CYPRINIF. (Siluroid.) Bagrid.

Selene de LACEPEDE 1802: P.PERCIF. (Percoid.) Carangid.

Selenidera GOULD 1834: Av. PICIF. Rhamphastid.

Selenotoca MYERS 1836: P.-CYPRINIF. (Cyprinoid.) Cyprinid.

Seleucidis LESSON 1834 : Av. PASSERIF. (Acromyod.) Paradiseid. (Paradisein.).

Selevinia BELOSLUDOV et BASHANAV 1939 : M.-RODENT. (Myomorph.) Glirid. (Glirin.).

Selysius BONAPARTE 1841: s./g. de Myotis KAUP 1829.

Sema JORDAN 1878: P.-PERCIF. (Labroid.) Embiotocid.

Semablennius FOWLER 1954 : P.PERCIF. (Blennioid.) Blenniid.

Semachlorella FOWLER et BEAN $1928: \mathrm{s} . / \mathrm{g}$. de Novaculichthys BLEEKER 1862.

Semadascyllus FOWLER 1941 : P.PERCIF. (Labroid.) Pomacentrid.
Semaprochilodus FOWLER 1941 : P.-CYPRINIF. (Characoid.) Characid.

Semathunnus FOWLER 1933 : P.PERCIF. (Scombroid.) Thunnid.

Semeiophorus GOULD 1838: Av. CAPRIMULGIF. Caprimulgid.

Semelcarinata : P.-CYPRINIF. (Characoid.) Characid.

Semicossyphus GUNTHER 1861 : P.-PERCIF. (Labroid.) Labrid.

Semiculter CHU 1935 : P.-CYPRINIF. (Cyprinoid.) Cyprinid.

Semilabeo PETERS 1880 : P.-CYPRINIF. (Cyprinoid.) Cyprinid.

Semimerula SCLATER 1859: Av. PASSERIF. (Acromyod.) Turdid. (Turdin.).

Seminatrix COPE 1895 : R.-SQUAMAT. (Serpent.) Colubrid.

Semiontera GRAY 1859: Av. PASSERIF. (Acromyod.) Paradiseid. (Paradisein.).

Semiplotus BLEEKER 1863: P.CYPRINIF, (Cyprinoid.) Cyprinid.

Semitapicis EIGENMANN et EINGENMANN 1889: P. - CYPRINIF. (Characoid.) Characid.

Semnodactylus HOFFMAN 1939 : Amph. ANOUR. Ranid. ou Bufonid.

Semnopithecus CUVIER 1821 : M.PRIMAT. (Simioid.) Cercopithecid. (Colobin.).

Semnopithecus DESMAREST 1822: syn. de Semnopithecus CUVIER 1921 nec 1825.

Semotilus RAFINESQUE 1820 : P.-CYPRINIF. (Cyprinoid.) Cyprinid.

Senex GRAY 1838: syn. de Phalcobaenus d'ORBIGNY 1835.

Seniocebus GRAY 1870: s./g. de Leontocebus WAGNER 1839.

Senonycteris GRAY 1870 : syn. de Rousettus GRAY 1821.

Sepacontias GUNTHER 1880 : R.SQUAMAT. (Saur.) Scincid.

Sepedon MERREM 1820 : R.-SQUAMAT. (Serpent.) Elapid.

Sepedonophis SMITH 1936 : R.SQUAMAT. (Serpent.) Boigid. 
Sepophis BOULENGER 1887: R.SQUAMAT. (Saur.) Scincid.

Seps LAURENTI 1768: syn. de Chalcides LAURENTI 1768.

Sepsina BOCAGE 1860: syn. de Scelotes FITZINGER 1826.

Septobranchus HARDENBERG 1941 : P.-CYPRINIF. (Siluroid.) Bagrid.

Sericagobioides HERRE 1927 : P.PERCIF. (Gobioid.) Eleotrid.

Sericonycteris MATSCHIE 1899 : syn. de Pteropus BRISSON 1762.

Sericornis GOULD 1838 : Av. PAS SERIF. (Acromyod.) Sylviid. ou Timaliid.

Sericossypha LESSON 1844: Av. PASSERIF. (Acromyod.) Tanagrid.

Sericotes REICHENBACH 1854 : Av. APODIF. Trochilid.

Sericulus SWAINSON 1825: Av. PASSERIF. (Acromyod.) Paradiseid. (Ptylonorhynchin.).

Serilophus SWAINSON 1837: Av. PASSERIF. (Desmodactyl.) Eurylaimid.

Serinops ROBERTS 1922: Av. PASSERIF. (Acromyod.) Fringillid. (Fringillin.).

Serinopsis RIDGWAY 1846: Av. PASSERIF. (Acromyod.) Fringillid. (Fringillin.).

Serinus BRISSON 1760: Av, PASSERIF. (Acromyod.) Fringillid. (Fringillin.).

Seriola CUVIER 1817 : P.-PERCIF. (Percoid.) Carangid.

Seriolella GUICHENOT 1849 : P.PERCIF. (Stromateoid.) Stromateid.

Seriolichthys BLEEKER 1854 : P.PERCIF. (Percoid.) Carangid.

Seriolina WAKIYA 1924 : P.-PERCIF. (Percoid.) Carangid.

Seriolophus GUICHENOT 1867 : P.-PERCIF. (Percoid.) Carangid.

Seriphus AYRES 1860 : P.-PERCIF. (Percoid.) Sciaenid.

Serpe RISSO 1810 : P.-CLUPEIF (Myctophoid.) Myctophiid.

Serpentarius CUVIER 1798: syn. de Sagittarius HERMANN 1783.
Serphophaga AGASSIZ 1845 : emend. pro Serpophaga GOULD 1839.

Serpophaga GOULD 1839: Av. PASSERIF. (Mesomyod.) Tyrannid. (Platyrhinchin.).

Serranellus JORDAN et EIGENMANN 1890 : P.-PERCIF. (Percoid.) Serranid.

Serranguilla WHITLEY et PHILLIPPS 1939 : P.-ANGUILLIF. (Anguilloid.) Muraenid.

Serranichthys BLEEKER 1855 : P.PERCIF. (Percoid.) Serranid.

Serraniculus GINSBURG 1952:P. PERCIF. (Percoid.) Serranid.

Serranochromus REGAN 1920 : $P$. PERCIF. (Percoid.) Cichlid.

Serranops REGAN 1914 : P.-PERCIF. (Percoid.) Serranid.

Serranus CUVIER 1817: P.-PERCIF. (Percoid.) Serranid.

Serraria GILBERT 1884: P.-PERCIF. (Percoid.) Etheostomid.

Serrasalmo DUMERIL 1805 : emend. pro Serrasalmus de LACEPEDE 1803.

Serrasalmus de LACEPEDE 1803 : P.-CYPRINIF. (Characoid.) Characid.

Serrator SPRUNGLI 1784 : syn. de Mergus LINNE 1758.

Serresius BONAPARTE 1855: Av. COLUMBIF. Columbid. (Treronin.).

Serrichromis FOWLER 1943: $P$. PERCIF. (Labroid.) Pomacentrid.

Serrihastaperca FOWLER 1944 : P.-PERCIF, (Percoid.) Serranid.

Serrivomer GILL et RYDER 1883 : P. - ANGUILLIF. (Nemichthyoid.) Serrivomerid.

Serval BREHM 1864 : syn. de Felis LINNE 1758.

Servalina GREVE 1897: syn. de Felis LINNE 1758.

Setarches JOHNSON 1862 : P.-PERCIF. (Scorpaenoid.) Scorpaenid.

Setaria BLYTH 1844: Av. PASSERIF. (Acromyod.) Timaliid.

Setifer (Froriep) DUMERIL 1806 : M.-INSECTIV. Tenrecid. (Tenrecin.). 
Setipinna SWAINSON 1839 : P.CLUPEIF. (Clupeoid.) Engraulid.

Setochalcis OBERHOLSER 1914 : Av. CAPRIMULGIF. Caprimulgid.

Setonyx LESSON 1842: M.-MARSUP. Macropodid. (Macropodin.).

Setopagis RIDGWAY 1912: Av. CAPRIMULGIF. Caprimulgid. (Caprimulgin.).

Setophaga SWAINSON 1827: Av. PASSERIF. (Acromyod.) Parulid.

Setosura MATHEWS 1913 : syn. de Rhipidura VIGORS et HORSFIELD 1826.

Settula: vide Cittura KAUP 1848.

Seweallia HORA 1932: P.-CYPRINIF. (Cyprinoid.) Homalopterid.

Seychellea SMITH 1957: P.-PERCIF. (Gobioid.) Gobiid.

Shacra BLEEKER 1860 : P.-CYPRINIF. (Cyprinoid.) Cyprinid.

Sheppardia HAAGNER 1909: Av. PASSERIF. (Acromyod.) Turdid.

Shinosaurus MELL 1930 : R.-SQUAMAT. (Saur.) Xenosaurid.

Shipwayia WHITLEY 1954 : P.PERCIF. (Gobioid.) Eleotrid.

Sherva VANZOLINI 1951: syn. de Zygaspis COPE 1885.

Siaga GRAY 1843 : errat, pro Saiga GRAY 1843.

Siagonodon PETERS 1881: R.SQUAMAT. (Serpent.) Leptotyphlopid.

Siaja BLEEKER 1860 : P.-CYPRINIF. (Cyprinoid.) Cyprinid.

Sialia SWAINSON 1827: Av. PASSERIF. (Acromyod.) Turdid. (Sialin.).

Sialis de LAFRESNAYE 1839 : syn. de Sialia SWAINSON 1827.

Siaphos GRAY 1839 : R.-SQUAMAT. (Saur.) Scincid.

Sibbaldus GRAY 1864 : syn. de Balaenoptera de LACEPEDE 1804.

Sibia HODGSON 1836 : Av. PASSERIF. (Acromyod.) Timaliid.
Sibilatrix KAUP 1829: s./g. de Phylloscopus BOIE 1826.

Sica TROUESSART 1898: emend. pro Sika SCLATER 1870.

Sicalis BOIE 1828: Av. PASSERIF. (Acromyod.) Fringillid.

Sicamugil FOWLER 1939 : P.-MUGILIF. Mugilid.

Sicista GRAY 1827: M.-RODENT. (Myomorph.) Zapodid. (Sicistin.).

Sicya JORDAN et EVERMANN 1896: syn. de Sicyosus JORDAN et EVERMANN 1898.

Sicyastes MULLER et TROSCHEL 1843 : P. -PERCIF. (Gobiesoxoid.) Gobiesocid.

Sicydium CUVIER et VALENCIENNES 1837: P.PERCIF. (Gobioid.) Gobiid.

Sicyogaster BRISOUT 1846 : P.PERCIF. (Gobiesoxoid.) Gobiesocid.

Sicyopterus GILL 1860 : P.-PERCIF. (Gobioid.) Gobiid.

Sicyopus GILL 1864: P.-PERCIF. (Gobioid.) Gobiid.

Sicyosus JORDAN et EVERMANN 1898: P.-PERCIF. (Gobioid.) Gobiid.

Sidera KAUP : P.-ANGUILLIF. (Anguilloid.) Muraenid.

Sideroderma PETERS 1871: syn. d'Hipposideros GRAY 1831.

Siebenrockiella LINDHOLM 1929 : R.-TESTUDIN. (Thecoph.) Emydid.

Sieboldia BONAPARTE 1838 : syn. de Megalobatrachus TSCHUDI 1837.

Sieboldornis MOMIYAMA 1928 : Av. PASSERIF. (Acromyod.) Bombycillid.

Sierra FOWLER 1905 : P.-PERCIF. (Scombroid.) Scombrid.

Siganites FOWLER 1904 : P.-PERCIF. (Siganoid.) Siganid.

Siganus FORSKALL 1775 : P.-PERCIF. (Siganoid.) Siganid. (serait non valide et syn. de Amphacanthus SCHNEIDER 1801).

Sigelus CABANIS 1851: Av. PASSERIF. (Acromyod.) Muscicapid. 
Sigmistes JORDAN et EVERMANN 1898 : P.-PERCIF. (Cottoid.) Cottid.

Sigmodon SAY et ORD 1825: M.RODENT. (Myomorph.) Cricetid. (Cricetin.).

Sigmodontomys ALLEN 1897 : syn. de Nectomys PETERS 1861.

Sigmodus BONAPARTE 1850 : Av. PASSERIF. (Acromyod.) Prionopid.

Sigmomys THOMAS 1901: M.-RODENT. (Myomorph.) Cricetid. (Cricetin.).

Sigmops GILL 1883 : P.-CLUPEIF. (Stomiatoid.) Gonostomid.

Sigmurus JORDAN et EVERMANN 1898: P.-PERCIF. (Percoid.) Sciaenid.

Signalosa EVERMANN et KENDALL 1898 : P.-CLUPEIF. (Clupeoid.) Dorosomid.

Sika SCLATER 1870: M.-PARAXON. Cervid.

Sikaillus HEUDE 1898: syn. de Cervus LINNE 1758.

Sikukia SMITH 1931: P.-CYPRINIF. (Cyprinoid.) Cyprinid.

Silenus GOLDFUSS 1820 : syn. de Macaca de LACEPEDE 1799.

Sillaginichthys BLEEKER 1876 : P.-PERCIF. (Percoid.) Latilid.

Sillaginodes GILL 1862: P.-PERCIF. (Percoid.) Latilid.

Sillaginoides: vide Sillaginodes GILL 1862.

Sillaginopodys FOWLER 1933 : P.PERCIF. (Percoid.) Latilid.

Sillaginopsis GILL 1862: P.-PERCIF. (Percoid.) Latilid.

Sillago CUVIER 1817 : P.-PERCIF (Percoid.) Latilid. (ou Sillaginid.).

Sillasomus : Av. PASSERIF. (Mesomyod.) Furnariid.

Silonia SWAINSON 1838: P.-CYPRINIF. (Siluroid.) Schilbeid.

Silonopangasius HORA : P.-CYPRINIF. (Siluroid.) Schilbeid.

Silubosaurus GRAY 1845: R.-SQUAMAT. (Saur.) Scincid.

Silundia CUVIER et VALENCIENNES 1840 : P.-CYPRINIF. (Siluroid.) Schilbeid.
Siluranodon BLEEKER 1858 : P.CYPRINIF. (Siluroid.) Schilbeid.

Silurichthys BLEEKER 1856 : P.CYPRINIF. (Siluroid.) Silurid.

Silurodes BLEEKER 1858 : P.-CYPRINIF. (Siluroid.) Silurid.

Silurus LINNE 1758 : P.-CYPRINIF. (Siluroid.) Silurid.

Silus GILL 1863: P.-CLUPEIF. (Opisthoproctoid.) Argentinid.

Silvesterina FOWLER 1934: P.ANGUILLIF. (Congroid.) Congrid.

Silybura PETERS 1861 : R.-SQUAMAT. (Serpent.) Uropeltid.

Simenchelys GOODE et BEAN 1879: P. - ANGUILLIF. (Anguilloid.) Simenchelyid.

Simia LINNE 1758 : syn. de Macaca de LACEPEDE 1799.

Simias MILLER 1903 : M.-PRIMAT. (Simioid.) Cercopithecid. (Colobin.).

Simobrama F O WLER 1944 : P. PERCIF. (Stromateoid.) Stromateid.

Simocantharus FOWLER 1933 : s./g. de Spondyliosoma CANTOR 1850.

Simocephalus GUNTHER 1858 : R.SQUAMAT. (Serpent.) Colubrid.

Simochromis BOULENGER 1898 : P.-PERCIF. (Percoid.) Cichlid.

Simocyon WAGNER 1858: syn. de Speothos LUND 1839.

Simophis PETER 1860 : R.-SQUAMAT. (Serpent.) Colubrid.

Simopias GILL 1905 : P.-PERCIF. (Scorpaenoid.) Synancejid.

Simorhynchus MERREM 1819: Av. ALCIF. Alcid. (Fraterculin.).

Simosciurus ALLEN 1915 : syn. de Sciurus LINNE 1758.

Simosyngnathus FOWLER 1940 : P.-SŸNGNATHIF. Syngnathid.

Simotes DUMERIL et BIBRON 1854 : R.-SQUAMAT. (Serpent.) Colubrid.

Simoxenops CHAPMAN 1937: Av. PASSERIF. (Mesomyod.) Furnariid.

Sineleotris HERRE 1940 : P.-PERCIF. (Gobioid.) Eleotrid. 
Sinibarbus SAUVAGE 1874 : P.CYPRINIF. (Cyprinoid.) Cyprinid.

Sinibrama WU 1939 : P.-CYPRINIF. (Cyprinoid.) Cyprinid.

Sinilabeo RENDAHL 1933 : P.-CYPRINIF. (Cyprinoid.) Cyprinid.

Siniperca GILL 1863 : P.-PERCIF. (Percoid.) Serranid.

Sinipercina : P.-PERCIF. (Percoid.) Serranid.

Sinisus HEUDE 1892 : syn. de Sus LINNE 1758.

Sinocyclocheilus FANG 1936: P.CYPRINIF. (Cyprinoid.) Cyprinid.

Sinoetherus CUVIER 1825 : syn. de Coendou de LACEPEDE 1799.

Sinogastromyzon FANG 1930 : P.CYPRINIF. (Cyprinoid.) Homalopterid.

Sinogobio: P.-CYPRINIF. (Cyprinoid.) Cyprinid.

Sinogobius LIU $1940: s . / g$. de Gobius LINNE 1758.

Sinomyrus LIN 1933 : P.-ANGUILLIF. (Congroid.) Echelid.

Sinosturio JAEKEL 1929: P.-ACIPENSERIF. Acipenserid.

Sinotes : R.-SQUAMAT. (Serpent.) Colubrid.

Siokunichthys HERALD 1953 : P.SYNGNATHIF. Syngnathid.

Siphagonus STEINDACHNER 1876: P.-PERCIF. (Cottoid.) Agonid.

Siphamia WEBER 1909: P.-PERCIF. (Percoid.) Serranid.

Siphateles COPE 1883 : P.-CYPRINIF. (Cyprinoid.) Cyprinid.

Siphia HODGSON 1837: Av. PASSERIF. (Acromyod.) Muscicapid.

Siphneus BRANDT 1827: syn. de Myospalax LAXMANN 1769.

Siphonochromis FOWLER 1946 : P.-PERCIF. (Labroid.) Pomacentrid.

Siphonognathus RICHARDSON 1858: P.-PERCIF. (Labroid.) Odacid.

Siphonops WAGLER 1828 : Amph. $A P O D$. Caeciliid.

Siphonorhis SCLATER 1861: Av. CAPRIMULGIF. Caprimulgid. (Caprimulgin.).
Siphonostoma KAUP 1856 : P.SYNGNATHIF. Syngnathid.

Siphostoma RAFINESQUE 1810 : s./g. de Syngnathus LINNE 1758.

Sipodus MATHEWS 1928 : Av. PASSERIF. (Acromyod.) Muscicapid.

Siptornis REICHENBACH 1853 : Av. PASSERIF. (Mesomyod.) Furnariid.

Sirembo BLEEKER 1858 : P.-PERCIF. (Ophidioid.) Brotulid. (serait syn. de Dicromita GOODE et BEAN 1895).

Siren LINNE 1766 : Amph. UROD. Sirenid.

Sirenodon WIEGMANN 1832 : formes larvaires d'Ambystomatid.

Siryctes CABANIS et HEINE 1890 : Av. PASSERIF. (Mesomyod.) Tyrannid. (Tyrannin.).

Sisopygis CABANIS et HEINE 1860 : Av. PASSERIF. (Mesomyod.) Tyrannid. (Fluvicolin.).

Sisor HAMILTON - B UCH A NAN 1822 : P.-CYPRINIF. (Siluroid.) Loricariid.

Sistrurus GARMAN 1883 : R.-SQUAMAT. (Serpent.) Crotalid.

Sisura STRICKLAND 1841: vide Seisura VIGORS et HORSFIELD 1826.

Sitagra REICHENBACH 1850:Av. PASSERIF. (Acromyod.) Ploceid. (Plocein.).

Sitagroides : Av. PASSERIF. (Acromyod.) Ploceid.

Sitana CUVIER 1829: R.-SQUAMAT. (Saur.) Agamid.

Sitomys FITZINGER 1867 : syn. de Peromyscus GLOGER 1841.

Sitta LINNE 1758: Av. PASSERIF. (Acromyod.) Sittid.

Sittace WAGLER 1832: syn. de Psittacus LINNE 1758.

Sittasomus SWAINSON 1827: Av. PASSERIF. (Mesomyod.) Dendrocolaptid.

Sittella RAFINESQUE 1815 : emend. pro Sitta LINNE 1758.

Sittiparus SELYS 1884: s./g. de Parus LINNE 1758.

Siva HODGSON 1838: Av. PASSERIF. (Acromyod.) Timaliid. 
Skagerakia NYBELIN 1947: P.CLUPEIF. (Myctophoid.) Bathypteroid.

Skeponopodus NARDO 1832 : P.PERCIF. (Scombroid.) Istiophorid.

Skiffia MEEK 1902 : P.-CYPRINODONTIF. Goodeid.

Sladenia REGAN 1908: P.-LOPHIIF. (Lophioid.) Lophiid.

Slatinia WERNER 1906 : P.-CYPRINIF. (Siluroid.) Loricariid.

Smaragdites BOIE 1831: syn. de Polytmus BRISSON 1760.

Smaragditis REICHENBACH 1849 : emend. pro Smaragdites BOIE 1831.

Smaragdochrysis GOULD 1861: Av. APODIF. Trochilid.

Smaragdolanius GRISCOM 1930 : Av. PASSERIF. (Acromyod.) Vireoid.

Smaragdus POEY 1860 : P.-PERCIF. (Gobioid.) Gobiid.

Smaris CUVIER 1814 : P.-PERCIF. (Percoid.) Maenid.

Smariscus : P.-PERCIF. (Percoid.) Maenid.

Smecticus VALENCIENNES 1855 : P.-PERCIF. (Percoid.) Serranid.

Smicrornis GOULD 1842 : Av. PASSERIF. (Acromyod.) Muscicapid.

Smiliogaster BLEEKER 1860 : P.CYPRINIF. (Cyprinoid.) Cyprinid.

Smilisca COPE 1865: Amph. ANOUR. Hylid.

Smilogobius HERRE 1934 : P.-PERCIF. (Gobioid.) Gobiid.

Smilorhis SUNDEVALL 1873: Av. PICIF. Capitonid.

Sminthillus BARBOUR et NOBLE 1920 : Amph. ANOUR. Brachycephalid.

Sminthopsis THOMAS 1887: M.MARSUP. Dasyurid. (Phascogalin.).

Sminthus NATHUSIUS 1840 : syn. de Sicista GRAY 1827.

Smithichthys HUBBS 1952: P.PERCIF. (Blennioid.) Clinid.

Smithiglaux BONAPARTE 1854:
Av. STRIGIF. Strigid. (Bubonin.).

Smithites JORDAN et GILBERT 1919 : P.-CLUPEIF. (Clupeoid.) Dussumierid.

Smithornis BONAPARTE 1850: Av. PASSERIF. (Desmodactyl.) Eurylaimid.

Smutsia GRAY 1865 : syn. de Manis LINNE 1758.

Snellius : P.-CLUPEIF. (Stomiatoid.) Sternoptychid.

Snethlagea BERLEPSCH 1909 : Av. PASSERIF. (Mesomyod.) Tyrannid.

Snyderichthys MILLER 1945 : P.CYPRINIF. (Cyprinoid.) Cyprinid.

Snyderina JORDAN et STARKS 1901: P.-PERCIF. (Scorpaenoid.) Congiopid.

Socnopaea STORMER 1904 : P.-CYPRINIF. (Siluroid.) Bagrid.

Solanoia GRAY : M.-FISSIP. Viverrid. (Galidiin.).

Solea WALBAUM 1792 : P.-PLEURONECTIF. (Soleoid.) Soleid.

Solegnathus SWAINSON 1839 : P.SYNGNATHIF. Syngnathid. ou Solegnathid.

Soleichthys BLEEKER 1860 : syn. d'Aesopia KAUP 1858.

Solenichthys BLEEKER 1865 : syn. de Solenostomus de LACEPEDE 1803.

Solenodon BRANDT 1833 : M.-INSECTIV. Solenodontid.

Solenognathina : P.-SYNGNATHIF. Solenostomid.

Solenognathus A G ASSIZ 1846 : emend. pro Solegnathus SWAINSON 1839.

Solenomormyrus BLEEKER 1874 : P.-CLUPEIF. (Mormyroid.) Mormyrid.

Solenophallus AURICH : P.-PHALLOSTETHIF. Phallostethid.

Solenostomus de LACEPEDE 1803: P. -SYNGNATHIF. Solenostomid.

Solenostomatichthys $B L E E K E R$ 1875: P.SYNGNATHIF. Solenostomid.

Soleonasus EIGENMANN 1912 : P.PLEURONECTIF. (Soleoid.) Soleid, 
Soleotalpa GUNTHER 1862: P.PLEURONECTIF. (Soleo $i d$.) Soleid.

Solisorex THOMAS 1924: M.-INSECTIV. Soricid. (Crocidurin.).

Solivomer MILLER 1947: P.-CLUPEIF. (Myctophoid.) Myctophiid.

Solomys THOMAS 1922 : s./g. de Melomys THOMAS 1922.

Somateria LEACH 1819 : Av. ANATIF. Anatid. (Anatin.).

Somersia BEEBE et TEE-VAN 1934: P.-PERCIF. (Blennioid.) Blenniid.

Somileptus SWAINSON 1839 : P.CYPRINIF. (Cyprinoid.) Cobitid.

Somniosus LESUEUR 1818: P.SQUALIF. (Squalioid.) Scymnorhinid.

Somnispinax WHITLEY $1940: s . / g$. de Centrophorus KNER 1859.

Sonora BAIRD et GIRARD 1853: R.-SQUAMAT. (Serpent.) Boid. (Boin.).

Sooglossus BOULENGER 1906 : Amph. ANOUR. Pelobatid.

Sordelina PROCTER 1923: R.SQUAMAT. (Serpent.) Colubrid.

Sorella HARTLAUB 1880 : Av. PASSERIF. (Acromyod.) Ploceid. (Passerin.).

Sorex LINNE 1735 : M.-INSECTIV. Soricid. (Soricin.).

Sorexglis CUVIER et GEOFFROYST-HILAIRE 1821 : syn. de Tupaia RAFFLES 1821.

Soricidus ALTOBELLO 1927 : syn. de Sorex LINNE 1735.

Soriculus BLYTH 1854 : M.-INSECTIV. Soricid. (Soricin.).

Soroplex GLOGER 1842: syn. de Colaptes SWAINSON 1825.

Sorosichthys WHITLEY 1945 : P.BERYCIF. Berycid.

Sorsogona HERRE 1934: P.-PERCIF. (Scorpaenoid.) Platycephalid.

Sorubim SPIX 1829 : P.-CYPRINIF. (Siluroid.) Bagrid.

Sorubimichthys BLEEKER 1862 : P.-CYPRINIF, (Siluroid.) Bagrid.
Sosia VAILLANT 1902 : P.-CYPRINIF. (Siluroid.) Bagrid.

Sotalia GRAY 1906: M.-CETAC. (Odontocet.) Delphinid.

Sovichthys SCHULTZ 1944 : P.-CYPRINIF. (Siluroid.) Bagrid.

Spalacomys PETERS 1860 : syn. de Nesokia GRAY 1842.

Spalacopus WAGLER 1832 : M.-RODENT. (Caviomorph.) Echimyid. (Octodontin.).

Spalax GULDENSTAEDT 1770 : M.-RODENT. (Hystricomorph.) Spalacid.

Spalerosophis JAN 1865 : R.-SQUAMAT. (Serpent.) Colubrid.

Spanius GISTEL 1848 : P.-PLEURONECTIF. (Soleoid.) Soleid.

Sparactodon de ROCHEBRUNE 1880: P.-PERCIF. (Labroid.) Pomacentrid.

Sparidentex MUNRO 1948: P.-PERCIF. (Percoid.) Sparid.

Sparisoma SWAINSON 1839 : P.PERCIF. (Labroid.) Scarid.

Sparodon SMITH 1938: P.-PERCIF. (Percoid.) Sparid.

Sparopsis KNER 1868 : P.-PERCIF. (Percoid.) Sparid.

Spartonoica PETERS 1950: Av. PASSERIF. (Mesomyod.) Dendrocolaptid. ou Furnariid.

Sparus LINNE 1758: P.-PERCIF. (Percoid.) Sparid.

Sparvius VIEILLOT 1816: Av. FALCONIF. Falconid.

Spasma GRAY 1866 : syn. de Megaderma GEOFFROY-ST-HILAIRE 1810.

Spathodus BOULENGER 1900 : P.PERCIF. (Percoid.) Cichlid.

Spathoscalabotes BOULENGER 1885: R.-SQUAMAT. (Saur.) Geckonid.

Spathura GOULD 1849: Av. APODIF. Trochilid.

Spatula BOIE 1822: Av. ANATIF. Anatid. (Anatin.).

Spatularia SHAW 1804: syn. de Polyodon SCHNEIDER 1801.

Spatuloricaria SCHULTZ 1944 : P.CYPRINIF. (Siluroid.) Loricariid. 
Spectrum de LACEPEDE 1799 : syn. de Pteropus BRISSON 1762 .

Speculanas $v$ on BOETTICHER 1829: Av. ANATIF. Anatid. (Anatin.).

Spedon : R.-SQUAMAT. (Serpent.) Elapid.

Spelaeophryne AHL 1924 : Amph. ANOUR. Microhylid.

Spelaeornis DAVID et OUSTALET 1877 : Av. PASSERIF. (Acromyod.) Troglodytid.

Spelerpes RAFINESQUE 1832 : Amph. UROD. Plethodontid.

Speorifera GRAY 1866 : syn. d'Hipposideros GRAY 1831.

Speothos LUND 1839: M.-FISSIP. Canid. (Simocyonin.).

Speotyto GLOGER 1842 : Av. STRIGIF. Strigid. (Bubonin.).

Spermestes SWAINSON 1837: Av. PASSERIF. (Acromyod.) Ploceid. (Estrildin.).

Spermophaga JARDINE et SELBY 1836: Av. PASSERIF. (Acromyod.) Ploceid. (Estrildin.).

Spermophila SWAINSON 1827: Av. PASSERIF. (Acromyod.) Fringillid. (Pitylin.).

Spermophilopsis BLASIUS 1884 : M.-RODENT. (Sciuromorph.) Sciurid. (Sciurin.).

Spermophilus CUVIER 1825 : syn. de Citellus OKEN 1816.

Spermospiza GRAY 1840 : Av. PAS SERIF. (Acromyod.) Ploceid. (Plocein.).

Sphaenops WAGLER 1830 : syn. de Chalcides LAURENTI 1768.

Sphaerias MILLER 1906: M.-CHIROPT. (Megachiropt.) Pteropid. (Pteropin.).

Sphaerichthys CANESTRINI 1860 : P.-PERCIF. (Anabantoid.) Anabantid.

Sphaerodactylus WAGLER 1830 : R.-SQUAMAT. (Saur.) Geckonid. (Sphaerodactylin.).

Sphaerodon RUPPELL 1838: P.PERCIF. (Percoid.) Lutjanid.

Sphaeroides AGASSIZ 1846: P.TETRAODONTIF. (Tetraodontoid.) Tetraodontid. (vide Spheroides).
Sphaeronycteris PETERS 1882 : $s . / g$. de Centurio GRAY 1842.

Sphagebranchus BLOCH 1795 : P.ANGUILLIF. (Congroid.) Ophichthyid.

Sphagemacrurus FOWLER 1925: P. - PLEURONECTIF. (Psettodoid.) Psettodid.

Sphargis MERREM 1820: syn. de Dermatochelys WAGLER 1830 (Dermochelys de BLAINVILLE 1816).

Sphecotheres VIEILLOT 1816: Av. PASSERIF. (Acromyod.) Oriolid.

Sphenanthias WEBER 1913: P.PERCIF. (Percoid.) Serranid.

Sphenentogobius FOWLER 1940 : P.-PERCIF. (Gobioid.) Gobiid.

Spheniscus BRISSON 1760: Av. SPHENISCIF. Spheniscid.

Sphenocercus GRAY 1840 : Av, COLUMBIF. Columbid. (Treronin.).

Sphenocichla GODWIN-AUSTEN et WALDEN 1875: Av. PASSERIF. (Acromyod.) Troglodytid.

Sphenoeacus STRICKLAND 1841: Av. PASSERIF. (Acromyod.) Timaliid.

Sphenomorphus FITZINGER 1843 : R.-SQUAMAT. (Saur.) Scincid.

Sphenophryne PETERS et DORIA 1878 : Amph. ANOUR. Microhylid.

Sphenoproctus CABANIS et HEINE 1860 : syn. de Pampa REICHENBACH 1854.

Sphenorhynchus LICHTENSTEIN 1823: Av. ARDEIF. Ciconid.

Sphenosargus FOWLER $1940: \mathrm{s} . / \mathrm{g}$. de Salema JORDAN et EVERMANN 1896.

Sphenosoma FITZINGER 1843 : R.SQUAMAT. (Saur.) Scincid.

Sphenostoma GOULD 1838: Av. PASSERIF. (Acromyod.) Parid.

Sphenura LICHTENSTEIN 1820 : Av. PASSERIF. (Acromyod.) Timaliid.

Sphenurus SWAINSON 1837 : syn. de Treron VIEILLOT 1816.

Spheramia FOWLER et BEAN 1930: s./g. d'Amia GRONOV 1854 (Serranid.). 
Spheroides BOSC 1803 : P.-TETRAODONTIF. (Tetraodontoid.) Tetraodontid.

Sphiggurus CUVIER $1825:$ s./g. de Coendou de LACEPEDE 1799.

Sphoenohyla LUTZ et LUTZ 1938 : Amph. ANOUR. Hylid.

Sphyraena WALBAUM 1792: P.MUGILIF. Sphyraenid.

Sphyraenocharax FOWLER 1907 : P.-CYPRINIF. (Characoid.) Characid.

Sphyraenops POEY 1860 : P.-PERCIF. (Percoid.) Serranid.

Sphyrapicus BAIRD 1858: Av. PICIF. Picid. (Picin.).

Sphyrichthys THIENEMANN 1828 : P. - GALEIF. (Carcharhinoid.) Sphyrnid.

Sphyrna RAFINESQUE 1810 : P.GALEIF. (Carcharhinoid.) Sphyrnid.

Sphyrnias RAFINESQUE 1815 : P.GALEIF. (Carcharhinoid.) Sphyrnid.

Spicara RAFINESQUE 1810 : P.PERCIF. (Percoid.) Maenid.

Spilocorydon REICHENOW 1879 : Av. PASSERIF. (Acromyod.) Alaudid.

Spilogale GRAY 1865: M.-FISSIP. Mustelid. (Mephitin.).

Spiloglaux KAUP 1848: Av. STRIGIF. Strigid. (Bubonin.).

Spilopelia SUNDEVALL 1873 : syn. de Turtur BODDAERT 1783.

Spiloptila SUNDEVALL 1872: Av. PASSERIF. (Acromyod.) Timaliid.

Spilornis GRAY 1840 : Av. FALCONIF. Falconid. (Aquilin.).

Spilostomias REGAN et TREWAVAS 1930: s./g. d'Eustomias FILHOL 1884.

Spilotes WAGLER 1830 : R.-SQUAMAT. (Serpent.) Colubrid.

Spilotichthys FOWLER 1904: P.PERCIF. (Percoid.) Lutjanid.

Spinachia CUVIER 1817 : P.-GASTEROSTEIF. Gasterosteid.

Spinax CLOQUET 1816 : syn. d'Etmopterus RAFINESQUE 1810.

Spinibarbichthys OSHIMA 1926 :
P.-CYPRINIF. (Cyprinoid.) Cyprinid.

Spinibarbus OSHIMA 1919 : P.-CYPRINIF. (Cyprinoid.) Cyprinid.

Spiniphryne BERTELSEN 1951 : syn. de Bertelsenna WHITLEY 1954.

Spinirhombus OSHIMA 1927 : syn. de Pseudorhombus BLEEKER 1862.

Spinivomer GILL et RYDER 1883 : P. - A N GUILLIF. (Nemichthyoid.) Nemichthyid.

Spinoblennius HERRE 1935 : P.PERCIF. (Blennioid.) Blenniid.

Spintherobolus EIGENMANN 1911: P.-CYPRINIF. (Characoid.) Characid.

Spinus KOCH 1816 : syn. de Carduelis BRISSON 1760.

Spirinchus JORDAN et EVERMANN 1893 : P. - CLUPEIF. (Salmonoid.) Osmerid.

Spirlingus FATIO 1890 : P.-CYPRINIF. (Cyprinoid.) Cyprinid.

Spirobranchus CUVIER 1829 : P.PERCIF. (Anabantoid.) Anabantid.

Spiza BONAPARTE 1824 : Av. PASSERIF. (Acromyod.) Fringillid.

Spizaëtus VIEILLOT 1816: Av. FALCONIF. Falconid. (Aquilin.).

Spizalauda BLYTH 1855 : Av. PASSERIF. (Acromyod.) Alaudid.

Spizella BONAPARTE 1831: Av. PASSERIF. (Acromyod.) Fringillid. (Emberizin.).

Spiziapteryx KAUP 1851 : Av. FALCONIF. Falconid. (Falconin.).

Spiziastur KAUP 1848: Av. FALCONIF. Falconid. (Aquilin.).

Spizithornis OBERHOLSER 1920 : Av. PASSERIF. (Mesomyod.) Tyrannid. (Spizithornin.).

Spizixos BLYTH 1845: Av. PASSERIF. (Acromyod.) Pycnonotid.

Spizocorys SUNDEVALL 1872 : Av. PASSERIF. (Acromyod.) Alaudid.

Spizornis : vide Spizithornis OBERHOLSER 1920.

Spodiopsar SHARPE 1889 : syn. de Sturnus LINNE 1758. 
Spodiornis SCLATER 1866: syn. de Haplospiza CABANIS 1851.

Spondyliosoma CANTOR 1850 : syn. de Cantharus CUVIER 1817.

Spondylurus FITZINGER 1826 : syn. de Mabuya RAFINESQUE 1815.

Sporadinus BONAPARTE 1854 : Av. APODIF. Trochilid.

Sporaeginthus CABANIS 1851 : syn. d'Amandava BLYTH 1836.

Sporophila CABANIS 1844:Av. PASSERIF. (Acromyod.) Fringillid. (Fringillin.).

Sporopipes CABANIS 1842: Av. PASSERIF. (Acromyod.) Ploceid. (Plocein.).

Spratella CUVIER et VALENCIENNES 1847 : P.-CLUPEIF. (Clupeoid.) Clupeid.

Spratellicypris HERRE et MYERS 1931 : P. - CYPRINIF. (Cyprinoid.) Cyprinid.

Spratelloides BLEEKER 1851 : P.CLUPEIF. (Clupeoid.) Dussumierid.

Spratellomorpha BERTIN 1946 : P.-CLUPEIF. (Clupeoid.) Clupeid.

Sprattus GIRGENSHON 1846 : P.CLUPEIF. (Clupeoid.) Clupeid.

Spreo GRAY 1840 : Av. PASSERIF. (Acromyod.) Sturnid. (Graculin.).

Springeria BIGELOW et SCHROEDER 1951 : Anacanthobatid.

Squalidus DYBOWSKI 1872: P.CYPRINIF. (Cyprinoid.) Cyprinid.

Squaliobarbus GUNTHER 1868 : P.-CYPRINIF. (Cyprinoid.) Cyprinid.

Squaliolus SMITH et RADCLIFFE 1912: syn. d'Euprotomicrus GILL 1864.

Squalius BONAPARTE 1841 : P.CYPRINIF. (Cyprinoid.) Cyprinid.

Squalogadus GILBERT et HUBBS 1916 : P.-GADIF. Macrourid.

Squalomugil OGILBY 1908 : P.MUGILIF. Mugilid.

Squaloraja RILEY 1833 : P.-SQUALIF. (Squatinoid.) Squatinid.
Squalus LIN NE 1758 : syn. de Acanthias LEACH 1814 pp. et de Mustelus LINCK 1790 pp.

Squamatornis BAKER 1928: Av, PASSERIF. (Acromyod.) Pycnonotid.

Squamicilia MITTELMANN 1952 : R.-SQUAMAT. (Saur.) Scincid.

Squatarola LEACH 1816 : Av. CHARADRIIF. Charadriid. (Charadriin.).

Squatina DUMERIL 1806 : P.SQUALIF. (Squatinoid.) Squatinid.

Squatinoraia NARDO 1824 : P.-RAJIF, (Rhinobatoid.) Rhinobatid.

Stachycolobus ROCHEBRUNE 1887: syn. de Colobus ILLIGER 1811.

Stachyridopsis SHARPE 1883 : Av. PASSERIF. (Acromyod.) Timaliid.

Stachyris HODGSON 1844: Av. PASSERIF. (Acromyod.) Timaliid.

Stactocichla SHARPE 1883: Av. PASSERIF. (Acromyod.) Timaliid. (Turdoidin.).

Stactolaema MARSHALL 1870 : Av. PICIF. Capitonid.

Stalix JORDAN et SNYDER 1902 : P.-PERCIF. (Trachinoid.) Opisthognathid.

Staphida GOULD 1871: Av. PASSERIF. (Acromyod.) Timaliid.

Stappersia BOULENGER 1914 : P.PERCIF. (Percoid.) Cichlid.

Starksina FOWLER 1906 : P.CYPRINIF. (Characoid.) Characid.

Starna BONAPARTE 1838 : syn. de Perdix BRISSON 1760.

Starnoenas BONAPARTE 1838 : Av. COLUMBIF. Columbid. (Columbin.).

Stathmonotus BEAN 1885 : P.-PERCIF. (Blennioid.) Clinid.

Staurois COPE 1865: Amph. ANOUR, Ranid.

Staurotypus WAGLER 1838: R.TESTUDIN. (Thecoph.) Dermatemyid.

Steatocranus BOULENGER 1899 : P.-PERCIF. (Percoid.) Cichlid. 
Steatogenys BOULENGER 1898 : P.-CYPRINIF. (Gymnotoid.) Sternarchid.

Steatomys PETERS 1846 : M.-RODENT. (Myomorph.) Murid. (Dendromyin.).

Steatornis de HUMBOLT et BONPLAND 1814:Av. CAPRIMULGIF. Steatornithid.

Steganopleura REICHENBACH 1850: Av. PASSERIF. (Acromyod.) Ploceid. (Estrildin.).

Steganopus VIEILLOT 1819: Av. CHARADRIIF. Charadriid. (Phalaropin.).

Steganura REICHENBACH 1850 : Av. PASSERIF. (Acromyod.) Ploceid. (Viduin.).

Stegastes JENYNS 1840 : P.-PERCIF. (Labroid.) Pomacentrid.

Stegonotus DUMERIL et BIBRON 1854: R.-SQUAMAT. (Serpent.) Colubrid.

Stegophilus REINHARDT 1859 : P.CYPRINIF. (Siluroid.) Trichomycterid.

Stegostoma MULLER et HENLE 1837 : P. - GALEIF. (Isuroid.) Orectolobid.

Steindachnerella EIGENMANN 1897 : syn. de Steindachneria GOODE et BEAN 1888.

Steindachneria EIGENMANN et EIGENMANN 1888: syn. de Steidachneridion EIGENMANN et EIGENMANN 1919.

Steidachneria GOODE et BEAN 1888 : P.-GADIF. Macrourid.

Steindachneridion EIGENMANN et EIGENMANN 1919 : P.-CYPRINIF. (Siluroid.) Bagrid.

Steindachnerina FOWLER 1906 : P.-CYPRINIF, (Characoid.) Characid.

Steinegeria JORDAN et EVERMANN 1896: syn. de Taractes LOWE 1843.

Stelgidillas OBERHOLSER 1899 : Av. PASSERIF. (Acromyod.) Pycnonotid.

Stelgidonotus GILBERT et THOMP. SON 1905: P.-PERCIF. (Cottoid.) Cottid.

Stelgidopteryx BAIRD 1858: Av. PASSERIF. (Acromyod.) Hirundinid.
Stelgis JORDAN et STARKS 1896 : P.-PERCIF. (Cottoid.) Agonid.

Stelgis CRAMMER 1896 : syn. d'Acanthostelgis FOWLER 1958.

Stelgistrops HUBBS 1926 : syn. de Stelgistrum JORDAN et EVERMANN 1898.

Stelgistrum JORDAN et EVERMANN 1898: P.-PERCIF. (Cottoid.) Cottid.

Stellerina CRAMMER 1896 : P.PERCIF. (Cottoid.) Agonid.

Stellicarens JORDAN et EVERMANN 1898 : P.-PERCIF. (Percoid.) Sciaenid.

Stellifer OKEN 1817 : P.-PERCIF. (Percoid.) Sciaenid.

Stelliferus STARKS 1828 : P.-PERCIF. (Percoid.) Sciaenid.

Stellio LAURENTI 1768: syn. d'Agama DAUDIN 1802.

Stellistius JORDAN et TANAKA 1927 : P.-PERCIF. (Scorpaenoid.) Hexagrammid.

Stellula GOULD 1861: Av. APODIF. Trochilid.

Stelophorus : vide Stolephorus de LACEPEDE 1803.

Stemmatopus CUVIER 1826 : syn. de Cystophora NILSSON 1820.

Stemonidium GILBERT 1905: P.ANGUILLIF. (Nemichthyoid.) Serrivomerid.

Stemonolepis : P.-CLUPEIF. (Myctophoid.) Sudid.

Stemonosudis HARRY 1951: P.CLUPEIF. (Myctophoid.) Sudid.

Stenatherina SCHULTZ 1948: P.MUGILIF. Atherinid.

Stenella GRAY 1866: M.-CETAC. (Odontocet.) Delphinid.

Stenesthes JORDAN 1917 : P.-PERCIF. (Percoid.) Sparid.

Steno GRAY 1846: M.-CETAC. (Odontocet.) Delphinid.

Stenobrachius EIGENMANN et EIGENMANN 1890: P.-CLUPEIF. (Myctophoid.) Myctophiid.

Stenobrycon : P.-CYPRINIF. (Characoid.) Characid.

Stenocaulus OGILBY 1908 : P.-BELONIF. (Belonoid.) Belonid. 
Stenocephalemys FRICK 1914 : M.RODENT. (Myomorph.) Murid. (Murin.).

Stenocephalus MERCERAT 1891: M.-RODENT. (Myomorph.) Murid. (Murin.).

Stenocercus DUMERIL et BIBRON 1837: R. -SQUAMAT. (Saur.) Iguanid.

Stenocranius KASTSCHENKO 1901: s./g. de Microtus SCHRANK 1798.

Stenodactylus: Amph. ANOUR. Bufonid.

Stenodelphis GERVAIS 1847 : M.CETAC. (Odontocet.) Platanistid. (Stenodelphinin.).

Stenoderma OKEN 1816 : M.-CHIROPT. (Microchiropt.) Phyllostomatid. (Stenodermin.).

Stenodus RICHARDSON 1836 : P.CLUPEIF. (Salmonoid.) Salmonid.

Stenogobius BLEEKER 1878: P.PERCIF. (Gobioid.) Gobiid.

Stenomys THOMAS 1910 : syn. de Rattus FISCHER 1803.

Stenophis BOULENGER 1896 : R.SQUAMAT. (Serpent.) Boigid.

Stenopontistes MIRANDA-RIBEIRO 1936: syn. de Steno GRAY 1846.

Stenops ILLIGER 1811: syn. de Loris GEOFFROY-ST-HILAIRE 1796.

Stenopsis CASSIN : Av. CAPRIMULGIF. Caprimulgid. (Caprimulgin.).

Stenorhina DUMERIL et BIBRON 1854 : R.-SQUAMAT. (Serpent.) Boigid.

Stenoscolopsis FOWLER: syn. de Lycogenis KUHL et HASSELT 1830.

Stenostira CABANIS et BONAPARTE 1850: Av. PASSERIF. (Acromyod.) Muscicapid.

Stenotomus GILL 1865: P.-PERCIF. (Percoid.) Sparid.

Stentor GEOFFROY -ST - HILAIRE 1812 : syn. d'Alouatta de LACEPEDE 1800.

Stephanibyx REICHENBACH 1852 : Av. CHARADRIIF. Charadriid. (Charadriin.).
Stephanoaëtus SCLATER 1922 : syn. de Spizaëtus VIEILLOT 1816.

Stephanoberyx GILL 1883 : P.-STEPHANOBERYCIF. Stephanoberycid.

Stephanolepis GILL 1861 : P.-PERCIF. (Balistoid.) Balistid. ou Monacanthid.

Stephanophorus STRICKLAND 1841: Av. PASSERIF. (Acromyod.) Tanagrid.

Stercorarius BRIISON 1760: Av. LARIF. Stercorariid.

Stereochilus COPE 1869: Amph. UROD. Plethodontid.

Stereocyclops COPE 1869 : Amph. ANOUR. Microhylid.

Stereolepidella WHITLEY 1954 : P.-PERCIF. (Percoid.) Serranid.

Stereolepis CASIER: syn. de Stereolepidella WHITLEY 1954.

Stereolepis AYRES 1859 : P.-PERCIF. (Percoid.) Serranid.

Sterletus RAFINESQUE $1820: s . / g$. d'Acipenser LINNE 1758.

Sterna LINNE 1758: Av. LARIF. Larid. (Sternin.).

Sternarchella EIGENMANN 1905: P. - CYPRINIF. (Gymnotoid.) Sternarchid.

Sternarchogiton EIGENMANN 1905: P.-CYPRINIF. (Gymnotoid.) Gymnotid.

Sternarchorhamphus EIGENMANN 1905: P.-CYPRINIF. (Gymnotoid.) Sternarchid.

Sternarchorhynchus CASTELNAU 1855 : P.-CYPRINIF. (Gymnotoid.) Sternarchid.

Sternarchus SCHNEIDER 1801: P. - CYPRINIF. (Gymnotoid.) Sternarchid.

Sternias JORDAN et EVERMANN 1898 : P.-PERCIF. (Cottoid.) Cottid.

Sternoclyta GRAY 1855 : Av. APODIF. Trochilid.

Sternoptychides DOUGLAS-OGILBY 1888 : P.-CLUPEIF. (Stomiatoid.) Sternoptychid.

Sternopteryx : errat. pro Sternoptyx HERMANN 1781. 
Sternoptyx HERMANN 1781 : P.CLUPEIF. (Stomiatoid.) Sternoptychid.

Sternopygus MULLER 1846 : P.-CYPRINIF. (Gymnotoid.) Sternarchid.

Sternothaerus BELL 1825 : errat. pro Sternotherus GRAY 1825.

Sternotherus GRAY 1825 : R.-TESTUDIN. (Thecoph.) Pelomedusid.

Sternotremia NELSON 1876 : P.PERCOPSIF. Percopsid.

Sternula BOIE 1822: Av. LARIF. Larid. (Sternin.).

Stethaprion COPE 1870 : P.-CYPRINIF. (Characoid.) Characid.

Stethochaetus GUNTHER 1854 : syn. de Trichogaster SCHNEIDER 1801.

Stethojulis GUNTHER 1861: P.PERCIF. (Labroid.) Labrid.

Stethopristis GILBERT 1905: P.ZEIF. Zeid.

Stethopterus BLEEKER 1853 : P.ANGUILLIF. (Congroid.) Ophichthyid.

Stevardia GILL 1858 : P.-CYPRINIF. (Characoid.) Characid.

Sthenelides STEJNEGER 1884: Av. ANATIF. Anatid.

Sthenopus RICHARDSON 1843 : P.PERCIF. (Percoid.) Aploactid.

Stichaeopsis STEINDACHNER et KNER 1870 :P.-PERCIF. (Blenntoid.) Blenniid.

Stichaeus REINHARDT 1836 : P.PERCIF. (Blennioid.) Blenniid. ou Clinid.

Sticharium GUNTHER 1867: P.PERCIF. (Blennioid.) Blenniid.

Stichonodon EIGENMANN 1903 : P.-CYPRINIF. (Characoid.) Characid.

Stictapteryx IREDALE et MATHEWS 1926: Av. APTERYGIF. Apterygid.

Stictocarbo BONAPARTE 1855: Av. PELECANIF. Phalacrocoracid.

Stictomys THOMAS 1924 : syn. de Cuniculus BRISSON 1762.

Stictonetta REICHENBACH 1852 : Av. ANATIF. Anatid. (Anatin.).

Stictophorus POCOCK 1926: M.-
MARSUP. Dasyurid. (Dasyurin.).

Stictoptera REICHENBACH 1863 : Av. PASSERIF. (Acromyod.) Ploceid. (Estrildin.).

Stictospiza SHARPE 1890: Av. PASSERIF. (Acromyod.) Ploceid. (Estrildin.).

Stigmatogobius BLEEKER 1874: P.-PERCIF. (Gobioid.) Gobiid.

Stigmatonotus PETERS 1877 : P.PERCIF. (Percoid.) Serranid.

Stigmatopelia SUNDEVALL 1873 : syn. de Turtur BODDAERT 1783.

Stigmatopora KAUP 1853 : P.-SYNGNATHIF. Syngnathid.

Stigmatops GOULD 1865 : Av. PASSERIF. (Acromyod.) Meliphagid.

Stigmatura SCLATER et SALVADORI 1866: Av. PASSERIF. (Mesomyod.) Tyrannid. (Platyrhinin.).

Stigogenes: P.-CYPRINIF. (Siluroid.) Loricariid.

Stilbe de KAY 1842 : P.-CYPRINIF. (Cyprinoid.) Cyprinid.

Stilbiscus JORDAN et BOLLMAN 1889 : P.-ANGUILLIF. (Anguilloid.) Moringuid.

Stilbius GILL 1865 : errat. pro Stilbe de KAY 1842.

Stilosoma BROWN 1890 : R.-SQUAMAT. (Serpent.) Colubrid.

Stiltia BONAPARTE $1854: s . / g$. de Glareola BRISSON 1760.

Stipecampus WHITLEY 1948 : P.SYNGNATHIF. Syngnathid.

Stiphodon WEBER 1895 : P.-PERCIF. (Gobioid.) Gobiid.

Stiphrornis HARTLAUB 1855: Av. PASSERIF. (Acromyod.) Turdid. ou Timaliid.

Stipituropsis BERTONI 1901: Av. PASSERIF. (Acromyod.) Timaliid.

Stipiturus LESSON 1831 : Av. PASSERIF. (Acromyod.) Timaliid. ou Sylviid.

Stizorhina OBERHOLSER 1899 : Av. PASSERIF. (Acromyod.) Muscicapid.

Stizostedion RAFINESQUE 1820 : P.-PERCIF. (Percoid.) Percid. 
Stlengicottus BOLIN 1936 : P.-PERCIF. (Cottoid.) Cottid.

Stlengis JORDAN et STARKS 1904: P.-PERCIF. (Cottoid.) Cottid.

Stoasodon CANTOR 1850 : P.-RAJIF. (Dasyatoid.) Myliobatid.

Stochomys THOMAS $1926: \mathrm{s} . / \mathrm{g}$. de Rattus FISCHER 1803.

Stokellia WHITLEY 1955 : P.-CLUPEIF. (Salmonoid.) Osmerid.

Stolephorus de LACEPEDE 1803 : P.-CLUPEIF. (Clupeoid.) Engraulid. ou Dussumierid.

Stoliczkaia BOULENGER 1890 : R.SQUAMAT. (Serpent.) Colubrid.

Stolothrissa REGAN 1917 : P.-CLUPEIF. (Clupeoid.) Clupeid.

Stomatorhincus BOULENGER 1898: P.-CLUPEIF. (Mormyroid.) Mormyrid.

Stombus GRAVENHORST 1825 : Amph. ANOUR. Leptodactylid.

Stomianodon BLEEKER 1849 : P.CLUPEIF. (Stomiatoid.) Stomiatid.

Stomias CUVIER 1817 : P.-CLUPEIF. (Stomiatoid.) Stomiatid.

Stomiasunculus KAUP 1860: P.CLUPEIF. (Stomiatoid.) Stomiatid.

Stomioides PARR 1933: P.-CLUPEIF. (Stomiatoid.) Stomiatid.

Stomiopera REICHENBACH 1852 : Av. PASSERIF. (Acromyod.) Zosteropid.

Stomocatus BONAPARTE 1841 : P.-CYPRINIF. (Cyprinoid.) Catostomid.

Stomodon MITCHILL 1814 : P.GADIF. Gadid.

Stomogobius WHITLEY 1931 : syn. d'Orthostomus KNER 1868.

Stoniella FOWLER 1914: P.-CYPRINIF. (Siluroid.) Loricariid.

Stoparola BLYTH 1836: Av. PASSERIF. (Acromyod.) Muscicapid.

Storeria BAIRD et GIRARD 1843 : R.-SQUAMAT. (Serpent.) Colubrid.

Strabo KNER et STEINDACHNER 1866 : P.-MUGILIF. Atherinid.

Strabozebrias CHABANAUD 1943 : P.-PLEURONECTIF. (Soleoid.) Soleid.
Strachyrhidopsis SHARPE : vide Stachyridopsis OATES 1883.

Strachyris : vide Stachyris HODGSON 1844.

Strandichthys WHITLEY 1937 : P.PLEURONECTIF. (Soleoid.) Soleid.

Strepera LESSON 1831: Av. PASSERIF. (Acromyod.) Corvid.

Strephon GISTEL 1848: syn. de Brontes CUVIER et VALENCIENNES 1840.

Strepitovagus FRIEDMANN 1927 : s./g. de Molothrus SWAINSON 1832.

Strepsiceros FRISCH 1775 : M.-PARAXON. Bovid. (Tragelaphin.).

Strensilas ILLIGER 1811 : syn. d'Arenaria BRISSON 1760.

Streptoceryle BONAPARTE 1854 : Av. CORACIADIF. Alcedinid.

Streptopelia BONAPARTE 1854 : syn. de Turtur BODDAERT 1783.

Streptophorus DUMERIL et BIBRON 1854: R.-SQUAMAT. (Serpent.) Boigid.

Streptoprocne OBERHOLSER 1906: Av. CAPRIMULGIF. Caprimulgid.

Streptosaurus MITTLEMANN 1942 : R.-SQUAMAT. (Saur.) Iguanid.

Streptozogognathus OBERHOLSER : Av. APODIF. Apodid.

Stresemannia MEISE 1950: Av. PASSERIF. (Acromyod.) Meliphagid.

Strializa : P.-MUGILIF. Mugilid.

Strigiceps BONAPARTE 1831 : syn. de Circus de LACEPEDE 1799.

Strigops GRAY 1841: Av. PSITTACIF. Psittacid.

Strigopsis GRAY 1845: Av. PSITTACIF. Psittacid.

Stringops FINSCH 1867: syn. de Strigops GRAY 1841.

Stringopsis van der HOEVEN 1955 : syn. de Strigopsis GRAY 1845.

Strinsia RAFINESQUE 1810 : P.GADIF. Gadid.

Strix LINNE 1758: Av. STRIGIF. Strigid. (Strigin.).

Strobilurus WIEGMANN 1834 : R.SQUAMAT. (Saur.) Iguanid. 
Stromateoides BLEEKER 1851 : P.PERCIF. (Stromateoid.) Stromateid.

Stromateus LINNE 1758 : P.-PERCIF. (Stromateoid.) Stromateid.

Strongyloceros OWEN 1846: syn. de Cervus LINNE 1758.

Strongylura van HASSELT 1823 : P.-BELONIF. (Belonoid.) Belonid.

Strophidon Mc CLELLAND 1844 : P. - ANGUILLIF. (Anguilloid.) Muraenid.

Strophiurichthys FRASER-BRUNNER 1935: P.-TETRAODONTIF. (Ostracionoid.) Ostracionid.

Strophodus A G A S I Z 1838: P.PERCIF. (Percoid.) Lutjanid.

Struthidea GOULD 1837 : Av. PASSERIF. (Acromyod.) Corvid.

Struthio LINNE 1758: Av. STRUTHIONIF. Struthionid.

Stumpffia BOETTE GER 1881 : Amph. ANOUR. Microhylid.

Stupens WHITLEY 1954: P.CYPRINIF. (Characoid.) Chara. cid.

Stupidogobius AURICH 1938: P. PERCIF. (Gobioid.) Gobiid.

Sturio RAFINESQUE 1810 : syn. d'Acipenser LINNE 1758.

Sturisoma SWAINSON 1838: P.CYPRINIF. (Siluroid.) Loricariid.

Sturnella VIEILLOT 1816: Av. PASSERIF. (Acromyod.) Icterid. (Sturnellin.).

Sturnia LESSON 1837: Av, PAS. SERIF. (Acromyod.) Sturnid. (Sturnin.).

Sturnira GRAY 1842 : M.-CHIROPT. (Microchiropt.) Phyllostomatid. (Sturnirin.).

Sturnirops GOODWIN 1938: M.CHIROPT. (Microchiropt.) Phyllostomatid. (Sturnirin.).

Sturnopastor BLYTH 1843: $A v$. PASSERIF. (Acromyod.) Sturnid. (Sturnin.).

Sturnus LINNE 1758: Av. PASSERIF. (Acromyod.) Sturnid. (Sturnin.).

Stygicola GILL 1864 : P.-PERCIF. (Ophidioid.) Brotulid.
Stygnobrotula SMITH 1957 : P.PERCIF. (Ophidioid.) Brotulid.

Stygogenes GUNTHER 1864: P. CYPRINIF. (Siluroid.) Loricariid. ou Silurid.

Stylephorus SHAW 1791: P.-LAMPRIDIF. Lampridid. ou Stylephorid.

Stylocerus SMITH 1827: syn. de Muntiacus RAFINESQUE 1815.

Styloctenium MATSCHIE 1899 : M.CHIROPT. (Megachiropt.) Pteropid. (Pteropin.).

Stylodipus ALLEN 1925: syn. de Scirtopoda BRANDT 1844.

Stylophis B E R G 1901 : R.-SQUAMAT. (Serpent.) Colubrid.

Stylophorus BERTHOLD 1827 : errat. pro Stylephorus SHAW 1791.

Stylophthalmella ROULE et ANGEL 1930 : P.-CLUPEIF. (Stomiatoid.) Idiacanthid.

Stylophthalmoides SANZO 1915 : P.-CLUPEIF. (Stomiatoid.) Sternoptychid.

Stylophthalmus BRAUER 1902 : P.CLUPEIF. (Stomiatoid.) Sternoptychid.

Stypodon GARMAN 1881: P.-CYPRINIF. (Cyprinoid.) Cyprinid.

Suaheliornis NEUMANN 1920 : Av. PASSERIF. (Acromyod.) Timaliid. (Timaliin.).

Sublegatus SCLATER et SALVIN 1868: Av. PASSERIF. (Mesomyod.) Tyrannid. (Spizitornin.).

Submyiagra MATHEWS 1913 : syn. de Myiagra VIGORS et HORSFIELD 1826.

Sudis CUVIER 1817: syn. de Arapaima MULLER $1843 \mathrm{pp}$. et de Heterotis MULLER $1843 \mathrm{pp}$.

Sudis RAF IN ES QUE 1810:P. CLUPEIF. (Myctophoid.) Sudid.

Sufflamen JORDAN 1916 : P.-PERCIF. (Balistioid.) Balistid.

Suggrundus WHITLEY 1930 : P.PERCIF. (Scorpaenoid.) Platycephalid.

Suillomeles ALLEN et BARBOUR 1909: M.-MARSUP. Peramelid. 
Suiriria REICHENBACH 1850:Av. PASSERIF. (Mesomyod.) Tyrannid.

Sula BRISSON 1760 : Av. PELECANIF. Sulid.

Sumeriomys ARGYROPOULO 1933: syn. de Microtus SCHRANK 1798.

Suncus EHRENBERG 1833 : M.-INSECTIV. Soricid. (Crocidurin.).

Sundasciurus MOORE 1958: M.RODENT. (Sciuromorph.) Sciurid. (Sciurin.).

Sunkus WAGLER 1843 : vide Suncus EHRENBERG 1833.

Suprasinelepichthys : P.-CYPRINIF. (Characoid.) Characid.

Surdisorex THOMAS 1906: M.-INSECTIV. Soricid. (Crocidurin.).

Suricata DESMAREST 1804: M.FISSIP. Viverrid. (Herpestin.).

Surnia DUMERIL 1806 : Av. STRIGIF. Strigid. (Bubonin.).

Surniculoides ROBERTS 1922 : Av. CUCULLIF. Cucullid.

Surniculus LESSON 1830 : Av, CUCULLIF. Cucullid.

Suruga JORDAN et SNYDER 1901 : P.-PERCIF. (Gobioid.) Gobiid.

Sus LINNE 1758: M.-PARAXON. (Suif.) Suid. (Suin.).

Suthora HODGSON 1838 : syn. de Paradoxornis GOULD 1836.

Sutorectus WHITLEY 1939: P.GALIF. (Isuroid.) Orectolobid.

Sutoria NICHOLSON 1853: Av. PASSERIF. (Acromyod.) Timaliid.

Suttonia SMITH 1953 : P.-PERCIF. (Percoid.) Serranid.

Suya HODGSON 1836 : syn. de Prinia HORSFIELD 1821.

Swainia JORDAN et EVERMANN 1896 : P.-PERCIF. (Percoid.) Etheostomid.

Swinhoia GRAY 1866 : syn. de Balaenoptera de LACEPEDE 1804.

Syacium RANZAN1 1840 : P.-PLEURONECTIF. (Pleuronectoid.) Bothid.

Sycalis TSCHUDI 1844 : syn. de Sicalis BOIE 1828.

Sycobrotus CABANIS 1851: Av.
PASSERIF. (Acromyod.) Ploceid. (Plocein.).

Syconycteris MATSCHIE 1899 : syn. de Macroglossus CUVIER 1824.

Syletor JORDAN 1919 : P.-ANGUILLIF. (Congroid.) Ophichthyid.

Sylvaemus $O G N E V$ 1927: syn. d'A podemus KAUP 1829.

Sylvanus OKEN 1816 : syn. de Macaca de LACEPEDE 1799.

Sylvestrornis MATHEWS 1928: s./g. de Tricholimnas SHARPE 1893.

Sylvia SCOPOLI 1769 : Av. PASSERIF. (Acromyod.) Sylviid.

Sylvicapra OGILBY 1836 : M.-PARAXON. Bovid. (Cephalophin.).

Sylvietta de LAFRESNAYE 1839 : Av. PASSERIF. (Acromyod.) Sylviid:

Sylvilagus GRAY 1867 : M.-LAGOMORPH. Leporid. (Leporin.).

Sylviorthorhynchus GRAY 1845: Av. PASSERIF. (Mesomyod.) Furnariid. ou Dendrocolaptid.

Sylviparus BURTON 1836: Av. PASSERIF. (Acromyod.) Parid.

Sylvisorex THOMAS 1905: s./g. de Suncus EHRENBERG 1833.

Syma LESSON 1827: Av. CORACIADIF. Alcedinid.

Symboulichthys CHABANAUD 1927: P.-PLEURONECTIF. (Pleuronectoid.) Bothid.

Symbranchus MULLER 1841: P. SYMBRANCHIF. Symbranchid.

Symmetrurus JORDAN 1878: P.CYPRINIF. (Cyprinoid.) Cyprinid.

Symmorphus GOULD 1838: Av. PASSERIF. (Acromyod.) Campephagid.

Sympeltophis WERNER 1925 : R.SQUAMAT. (Serpent.) Boigid.

Symphalangus GLOGER $1841: \mathrm{s} . / \mathrm{g}$. de Hylobates ILLIGER 1811.

Symphemia RAFINESQUE 1819 : Av. CHARADRIIF. Charadriid. (Scolopacin.).

Sympheotis : Av. RALLIF. Otidid.

Symphimus COPE 1869 : R.-SQUAMAT. (Serpent.) Colubrid.

Symphodus RAFINESQUE 1810 : syn. de Crenilabrus OKEN 1817. 
Sympholis COPE 1862 : R.-SQUAMAT. (Serpent.) Colubrid.

Symphorus GUNTHER 1872: P.PERCIF. (Percoid.) Sparid.

Symphurus RAFINESQUE 1810 : P.-PLEURONECTIF. (Soleoid.) Cynoglossid.

Symphysanodon BLEEKER 1878 : P.-PERCIF. (Percoid.) Lutjanid.

Symphysodon HECKEL 1840 : P.PERCIF. (Percoid.) Cichlid.

Symphysoglyphus RIBEIRO 1913 : P.-PERCIF. (Percoid.) Sciaenid.

Symplectes SWAINSON 1837: Av. PASSERIF. (Acromyod.) Ploceid. (Plocein.).

Sympodoichthys FACCIOLA 1888 : P.-GADIF. Gadid.

Symposiachrus BONAPARTE 1854: sun. de Monarcha VIGORS et HORSFIELD 1826.

Symproptopterus COCCO 1885 : P.CLUPEIF. (Myctophoid.) Sudid.

Symptervoia MULLER et HENLE 1837 : P.-RAJIF. (Rajioid.) Rajid.

Synagris WALBAUM 1792 : P.-PERCIF. (Percoid.) Lutjanid.

Synagrops GUNTHER 1887: P.PERCIF. (Percoid.) Lutjanid.

Synallaxis VIEILLOT 1819: Av. PASSERIF. (Mesomyod.) Furnariid.

Synanceichthys BLEEKER 1863 : P.-PERCIF. (Scorpaenoid.) Synancejid.

Synanceja SCHNEIDER 1801: P.PERCIF. (Scorpaenoid.) Synancejid.

Synanchia SWAINSON 1839: syn. de Synanceja SCHNEIDER 1801.

Synancia AGASSIZ 1846 : syn. de Synanceja SCHNEIDER 1801.

Synancidium MULLER 1843: P.PERCIF. (Scorpaenoid.) Synancejid.

Synaphobranchus JOHSON 1862 : P.-ANGUILLIF. (Congroid.) Synaphobranchid.

Synanteretmus GOODE et BEAN
1896: P.-CLUPEIF. (Stomiatoid.) Bathypteroid.

Synaptolaemus MYERS 1950 : P.CYPRINIF. (Characoid.) Characid.

Synaptomys BAIRD 1857 : M.-RODENT. (Myomorph.) Microtid.

Synaptura CANTOR 1849 : $P$.PLEURONECTIF. (Soleoid.) Synapturid.

Synapturanus de CARVALHO 1954: Amph. ANOUR. Microhylid.

Synapturichthys CHABANAUD 1927 : P.-PLEURONECTIF. (Soleoid.). Synapturid.

Synchalinus COPE 1894 : R.-SQUAMAT. (Serpent.) Colubrid.

Synchiropus GILL 1859 : P.-PERCIF. (Callionymoid.) Callionymid.

Synchirus BEAN 1890 : P.-PERCIF. (Cottoia.) Cottid.

Synchismus GILL 1861: P.-GALEIF. (Isuroid.) Orectolobid.

Synclidopus CHABANAUD 1943 : P.-PLEURONECTIF. (Soleoid.) Solezd.

Syncrossus BLYTH 1860 : P.-CYPRINIF. (Cyprinoid.) Cobitid.

Syndactyla REICHENBACH 1853 : Av. PASSERIF. (Mesomyod.) Dendrocolaptid.

Syndesmotis PETERS 1871: M.CHIROPT. (Microchiropt.) Hipposiderid.

Synechoglanis GILL 1859 : P.-CYPRINIF. (Siluroid.) Bagrid.

Synechogobius GILL 1864 : P.-PERCIF. (Gobioid.) Gobiid.

Synechopterus NORMAN 1935 : P.PERCIF. (Percoid.) Serranid.

Syngnathina : P.-SYNGNATHIF. Syngnathid.

Syngnathoides BLEEKER 1851 : P.SYNGNATHIF. Syngnathid.

Syngnathus LINNE 1758 : P.-SYNGNATHIF. Syngnathid.

Synistius GILL 1863 : P.-PERCIF. (Percoid.) Leiognathid.

Synodontis CUVIER 1817 : P.-CYPRINIF. (Siluroid.) Bagrid.

Synodus GRONOV 1763: P.-CLUPEIF. (Myctophoid.) Synodid.

Ann. de Parasitologie, T. XXXVII, N ${ }^{\circ}$ 5-6. -1962. 
Synoicus GOULD 1843 : Av. PHASIANIF. Phasianid. (Phasianin.).

Synophis PERACCA 1896 : syn. de Diaphorolepis JAN 1863.

Synotus KEYSERLING et BLASIUS 1839: syn. de Barbastella GRAY 1821.

Syntheosciurus BANGS 1902 : M. RODENT. (Sciuromorph.) Sciurid. (Sciurin.).

Synthliborhamphus BRANDT 1837: Av. ALCIF. Alcid. (Fraterculin.).

Sypheotides LESSON 1839: Av. RALLIF. Otidid.

Sypheotis BONAPARTE 1854: Av. RALLIF. Otidid.

Syrictes JORDAN et EVERMANN 1927: P.-SYNGNATHIF. Syngnathid.

Syrigma RIDGWAY 1878: Av. ARDEIF. Ardeid.

Syrmaticus WAGLER 1832: Av. PHASIANIF. Phasianid. (Phasianin.).

Syrnium SAVIGNY 1809 : syn. de Strix LINNE 1758.

Syrrhaptes ILLIGER 1811: Av. COLUMBIF. (Pteroclidif.) Pteroclidid.

Syrrhina MULLER et HENLE 1841: s./g. de Rhinobatus LINCK 1790.

Syrrhophus COPE 1878: Amph. ANOUR. Bufonid.

Syrrhothonus CHABANAUD 1933 : P.-PERCIF. (Gobioid.) Gobiid.

Systellura RIDGWAY 1912: Av. CAPRIMULGIF. Caprimulgid.

Systomus Mc-CLELLAND 1839 : P. CYPRINIF. (Cyprinoid.) Cyprinid.

\section{$\mathbf{T}$}

Taccocua LESSON 1830: Av, CUCULLIF. Cucullid.

Tachornis GROSSE 1847 : Av. APODIF. Apodid.

Tachuris de LAFRESNAYE 1836: Av. PASSERIF. (Mesomyod.) Tyrannid.

Tachyboa : errat. pro Trachyboa PETERS 1860.
Tachycineta CABANIS 1851: Av. PASSERIF. (Acromyod.) Hirundinid.

Tachydromus OPPEL 1811 : R.SQUAMAT. (Saur.) Lacertid.

Tachyeres OWEN 1875: Av. ANATIF. Anatid. (Anatin.).

Tachygia MITTELMANN 1952 : R.SQUAMAT. (Saur.) Scincid.

Tachyglossus ILLIGER 1811: M.MONOTREM. Tachyglossid.

Tachymenis WIEGMANN 1835 : R.SQUAMAT. (Serpent.) Boigid.

Tachynautes OBERHOLSER 1905 : syn. de Cypsiurus LESSON 1843.

Tachyophis de ROCHEBRUNE 1885 : R.-SQUAMAT. (Serpent.) Colubrid.

Tachyophis MARTENS $1934: s . / g$. de Dendrelaphis BOULENGER 1890.

Tachyoryctes RUPPELL 1835 : M.RODENT. (Hystricomorph.) Rhizomyid.

Tachypetes VIEILLOT 1816: syn. de Fregata de LACEPEDE 1799.

Tachyphonus VIEILLOT 1816: Av. PASSERIF. (Acromyod.) Tanagrid.

Tachyplectes WOLTERS 1943 : s./g. de Coliuspasser RUPPELL 1840.

Tachysaurus GRAY 1845: R.SQUAMAT. (Saur.) Scincid.

Tachysurus de LACEPEDE 1803 : P. - CYPRINIF. (Siluroid.) Bagrid.

Tachytriorchis K A UP 1844: Av. FALCONIF. Falconid. (Buteonin.).

Tacoccua : vide Taccocua LESSON 1847.

Tactostoma BOLIN 1939 : P.-CLU PEIF. (Stomiatoid.) Stomiatid. (ou Photostomiatid.).

Taczanowskia STOLCMAN 1926 : syn. de Phrenotriccus RICHEMOND 1927.

Tadarida RAFINESQUE 1814: M.CHIROPT. (Microchiropt.) Molossid.

Tadorna OKEN 1817 : Av. ANATIF. Anatid. (Anatin.). 
Tadornes GRAY 1871 : syn. de Tadorna OKEN 1817.

Taenarichthvs WHITLEY 1953 : P.PERCIF. (Percoid.) Sparid.

Taenarus WHITLEY : syn. de Taenarichthys WHITLEY 1953.

Taendopygia : Av. PASSERIF. (Acromyod.) Ploceid.

Taenia ROSE 1793: P.-PERCIF. (Percoid.) Cepolid.

Taeniacara MYERS 1935 : P.-PERCIF. (Percoid.) Cichlid.

Taenianotus de LACEPEDE 1802: P. - PERCIF. (Scorpaenoid.) Scorpaenid.

Taeniaparadisea KINGHORN 1939: Av. PASSERIF. (Acromyod.) Paradiseid. (Paradisein.).

Taenioconger HERRE 1923: P.ANGUYLLIF. (Congroid.) Nettastotid.

Taenioides de LACEPEDE 1800 : P.-PERCIF. (Gobioid.) Gobiid.

Taeniogale GRAY 1864: syn. d'Herpestes ILLIGER 1811.

Taeniolabrus STEINDACHNER 1867: P.-PERCIF. (Trachinoid.) Pinguipedid.

Taeniomembras OGILBY 1898 : P.MUGILIF. Atherinid.

Taenionema EIGENMANN et BEAN 1907 : P.-CYPRINIF. (Siluroid.) Bagrid.

Taeniophis KAUP 1860: P.-ANGUILLIF. (Anguilloid.) Muraenid.

Taenionhorus BERTELSEN et MARSHALL 1956 : syn. d'Eutaeniophorus BERTELSEN et MARSHALL 1958.

Taeniopygia REICHENBACH 1863: Av. PASSERIF. (Acromyod.) Ploceid. (Estrildin.).

Taeniopsetta GILBERT 1905: P.PLEURONECTIF. (Pleuronectoid.) Bothid.

Taenioptera BONAPARTE 1830 : Av. PASSERIF. (Mesomyod.) Tyrannid. (Fluvicolin.).

Taeniopyga REICHENBACH 1862 : Av. PASSERIF. (Acromyod.) Ploceid.

Taeniotoca A G ASSIZ 1862 : P.PERCIF. (Labroid.) Embiotocid.
Taeniotriccus BERLEPSCH et HERTERT 1902: Av. PASSERIF. (Mesomyod.) Tyrannid.

Taeniura MULLER et HENLE 1837: P.-RAJIF. (Dasyatoid.) Dasyatid.

Taenopus MERRIAM 1903 : M.-RODENT. (Myomorph.) Cricetid. (Hesperomyin.).

Taeromys SODY 1941: M.-RODENT. (Myomorph.) Murid. (Murin.).

Tagusa HERRE 1935 : P.-PERCIF. (Blennioid.) Blenniti.

Taha REICHENBACH $1863: s . / g$. d'Euplectes SWAINSON 1829.

Taius JORDAN et THOMPSON 1912 : P.-PERCIF. (Percoid.) Sparid.

Takatsukasaia HACKISUKA 1934: Av. PASSERIF. (Acromyod.) Muscicapid.

Takydromus DAUDIN 1802 : syn. de Tachydromus OPPEL 1811.

Talaphorus MULSANT et VERREAUX 1874: Av. APODIF. Trochilid.

Talegallus LESSON 1826: Av. PHASIANIF. Megapodid.

Talismania GOODE et BEAN 1895 : s./g. de Bathytroctes GUNTHER 1878.

Talpa LINNE 1766 : M.-INSECTIV. Talpid. (Talpin.).

Tamandua RAFINESQUE 1815: M.-EDENT. (Xenarthr.) Myrmecophagid.

Tamanka HERRE 1927 : P.-PERCIF. (Gobioid.) Eleotrid.

Tamias ILLIGER 1811: M.-RODENT. (Sciuromorph.) Sciurid. (Sciurin.).

Tamiasciurus TROUESSART 1880: M. -RODENT. (Sciuromorph.) Sciurid. (Sciurin.).

Tamiodes POCOCK 1923 : syn. de Funambulus LESSON 1835.

Tamiops ALLEN $1906: \mathrm{s} . / \mathrm{g}$. de Callosciurus GRAY 1864 .

Tamiscus THOMAS 1918: syn. de Paraxerus FORSYTH-MAJOR 1893.

Tana LYON 1913 : syn. de Tupaia RAFFLES 1821 . 
Tanagra LINNE 1764: Av. PASSERIF. (Acromyod.) Tanagrid.

Tanagrella SWAINSON 1837: Av. PASSERIF. (Acromyod.) Tanagrid.

Tanagroides BONAPARTE 1831: Av. PASSERIF. (Acromyod.) Tanagrid.

Tanakia JORDAN et THOMPSON 1914: P.-CYPRINIF. (Cyprinoid.) Cyprinid.

Tanakius HUBBS 1918 : P.-PLEURONECTIF. (Pleuronectoid.) Pleuronectid.

Tandanus MITCHELL 1838 : P.-CYPRINIF. (Siluroid.) Plotosid.

Tandya WHITLEY 1930 : P.-PERCIF. (Trachinoid.) Opisthognathid.

Tanganicodus : P.-PERCIF. (Percoid.) Cichlid.

Tanganikallabes POLL 1943 : $P$.CYPRINIF. (Siluroid.) Clariid.

Tangara BRISSON 1760 : Av. PASSERIF. (Acromyod.) Tanagrid.

Tangavius LESSON 1839 : Av. PASSERIF. (Acromyod.) Icterid.

Tanichthys LIN 1932: P.-CYPRINIF. (Cyprinoid.) Cyprinid.

Tannayia RIBEIRO 1918: P.-CYPRINIF. (Siluroid.) Doradiá.

Tantalisor : P.-PERCIF. (Balistoid.) Balistid.

Tantalophis DUELLMAN 1958 : R.SQUAMAT. (Serpent.) Boigid.

Tantaleus REICHENBACH 1852: syn. de Plegadis KAUP 1829.

Tantalus LINNE 1758: Av. ARDEIF. Ciconid.

Tantilla BAIRD et GIRARD 1853 : R.-SQUAMAT. (Serpent.) Boigid.

Tantillita SMITH 1941 : R.-SQUAMAT. (Serpent.) Boigid.

Tanygnathus WAGLER 1832: Av. PSITTACIF. Psittacid.

Tanysiptera VIGORS 1825 : Av. CORACIADIF. Alcedinid.

Taoniscus GLOGER 1842: Av. TINAMIF. Tinamid.

Tapaia GRAY 1860 : syn. de Tupaia RAFFLES 1821.

Tapaja OKEN 1816 : syn. de Tupaia RAFFLES 1821.
Tapayia GRAY 1825: syn. de Tupaia RAFFLES 1821.

Tapaya FITZINGER 1825 : syn. de Phrynosoma WIEGMANN 1828.

Tapera BONAPARTE 1857: Av. CUCULLIF. Cucullid.

Tapetis GRAY 1867 : syn. de Sylvilagus GRAY 1867.

Taphonycteris DOBSON 1876 : syn. de Taphozous GEOFFROY-STHILAIRE 1812.

Taphozous GEOFFROY-ST-HILAIRE 1812: M.-CHIROPT. (Microchiropt.) Emballonurid.

Taphrometopon BRANDT 1838: R.-SQUAMAT. (Serpent.) Boigid.

Tapinophis BOULENGER 1899 : R.SQUAMAT. (Serpent.) Colubrid.

Tapinurus AMARAL 1932: R.SQUAMAT. (Saur.) Iguanid.

Tapir BLUMENBACH 1779: syn. de Tapirus BRISSON 1762.

Tapirella PALMER 1903: syn. d'Elasmognathus GILL 1865.

Tapirus BRISSON 1762 : M.-PERISSODACTYL. (Ceratomorph.) Tapirid.

Tapyra LIAIS 1872 : syn. de Tapirus BRISSON 1762.

Taraba LESSON 1831: Av. PASSERIF. (Mesomyod.) Formicariid.

Taractes LOWE 1843: P.-CORYPHAENIF. Bramid.

Taractichthys MEAD et MAUL 1958: P.-CORYPHAENIF. Bramid.

Tarandichthys JORDAN et EVERMANN 1896 : P.-PERCIF. (Cottoid.) Cottid.

Tarandus BILLBERG 1827 : syn. de Rangifer SMITH 1827.

Tarbophis FLEISCHMANN 1831: R.-SQUAMAT. (Serpent.) Boigid.

Tarentola GRAY 1825: R.-SQUAMAT. (Saur.) Geckonid.

Tardigradus BODDAERT 1785 : syn. de Loris GEOFFROY-STHILAIRE 1796.

Taricha GRAY 1850 : Amph. UROD. Salamandrid.

Tarimolagus : M.-LOGOMORPH. Leporid. (Leporin.). 
Tarletonbeania EIGENMANN et EIGENMANN 1891: P. $C L U$ PEIF. (Myctophoid.) Myctophiid.

Tarphops JORDAN et THOMPSON 1914.

Tarpon JORDAN et EVERMANN 1896 : P.-CLUPEIF. (Elopoid.) Elopid.

Tarsiger HODGSON 1844 : Av. PASSERIF. (Acromyod.) Turdid. (Turdin.).

Tarsipes GERVAIS 1842: M.-MARSUP. Phalangerid. (Tarsipedin.).

Tarsites JORDAN 1919 : P.-RAJIF. (Rhinobatoid.) Rhinobatid.

Tarsius STORR 1780: M.-PRIMAT. (Tarsioid.) Tarsiid.

Tarsomys MEARNS 1905: M.-RODENT. (Myomorph.) Murid. (Murin.).

Tasmanogobius SCOTT 1935 : P.PERCIF. (Gobioid.) Gobiid.

Tateichthys LAMONTE 1929 : P.CYPRINIF. (Gymnotoid.) Sternarchid.

Tatera LA TA S T E 1882 : M.-RODENT. (Myomorph.) Gerbillid.

Taterillus THOMAS 1910: M.-RODENT. (Myomorph.) Gerbillid.

Taterina WETTSTEIN 1916: syn. de Taterillus THOMAS 1910.

Taterona WROUGHTON 1917 : M.RODENT. (Myomorph.) Gerbillid.

Tateurndina NICHOLS 1955 : P.PERCIF. (Gobioid.) Eleotrid.

Tathicarpus OGILBY 1907 : P.-LOPHIIF. (Ogcocephaloid.) Ogcocephalid.

Tatia RIBEIRO 1912: P.-CYPRINIF. (Siluroid.) Doradid.

Tatu FRISCH 1775 : syn. de Dasypus LINNE 1758.

Tatusia LESSON 1827 : syn. de Dasypus LINNE 1758.

Tatusuia CUVIER: syn. de Dasypus LINNE 1758.

Taumakoides WH I T LE Y 1955 : $s . / g$. d'Acanthoclinus JENYNS 1841.

Tauphysa RENDHAL 1933 : P.-CYPRINIF. (Cyprinoid.) Cobitid.
Tauraco KLUK 1779 : syn. de Touraco de LACEPEDE 1799.

Tauredophidium ALCOCK 1890 : P.-PERCIF. (Ophidioid.) Brotulid.

Taurichthys CUVIER 1829 : P.PERCIF. (Percoid.) Chaetodontid.

Tauridea JORDAN et RICE 1878 : P.-PERCIF. (Cottoid.) Cottid.

Taurocottus SOLDATOV et PAVLENKO 1916 : P.-PERCIF. (Cottoid.) Cottid.

Taurotragus WAGNER 1855: M.PARAXON. Bovid. (Tragelaphin.).

Taurulus GRACIANOV 1907: P.PERCIF. (Cottoid.) Cottid.

Taurus RAFINESQUE 1814: syn. de Bos LINNE 1758.

Tautagus KLOSS 1917 : syn. de Mus LYNNE 1758.

Tautoga MITCHILL 1814 : P.-PERCIF. (Labroid.) Labrid.

Tautogolabrus GUNTHER 1862 : P.-PERCIF. (Labroid.) Labrid.

Taxidea WATERHOUSE 1839 : M.FISSIP. Mustelid. (Melin.).

Taxus CUVIER et GEOFFROY-STHILAIRE 1795 : syn. de Meles BRISSON 1762.

Tayassu FISCHER 1814: M.-PARAXON. (Suif.) Suid. (Dicotylin.).

Tayra OKEN 1816 : syn. de Galera (BROWNE) GRAY 1842.

Tchagra LESSON 1831: Av. PASSERIF. (Acromyod.) Laniid.

Tchagroides ROBERTS 1922: Av. PASSERIF. (Acromyod.) Laniid.

Tchitrea LESSON 1831: Av. PASSERIF. (Acromyod.) Muscicapid.

Taenopus MERRIAM 1903 : M.-RODENT. (Myomorph.) Cricetid. (Cricetin.).

Thecabteryx FOWLER 1948: P.TETRAODONTIF. (Tetraodontoid.) Tetraodontid.

Tectovaranus BLOESCH 1948 : s./g. de Varanus MERREM 1820.

Teixeirichthys SMITH 1953: P.PERCIF. (Labroid.) Pomacentrid. 
Tejus (MERREM) SCHINZ 1822: R.-SQUAMAT. (Saur.) Tejid.

Tekla NICHOLS 1922 : syn. de Paraclinus $M O C Q U A R D 1889$.

Telara GUNTHER 1868: P.-CLUPEIF. (Clupeoid.) Engraulid.

Telecanthura: Av. APODIF. Apodid.

Teleogramma BOULENGER 1899 : P.-PERCIF. (Percoid.) Cichlid.

Teleonema REICHENBACH 1850 : Av. PASSERIF. (Mesomyod.) Piprid.

Teleotrema REGAN et TREWAVAS 1932: P.-LOPHIIF. (Ceratoid.) Gigantactinid.

Teleovipera REUSS 1927: R.SQUAMAT. (Serpent.) Viperid.

Telescopias JORDAN et SNYDER 1901: P.-PERCIF. (Percoid.) Serranid.

Telescops B LEE K ER 1876 : P.PERCIF. (Percoid.) Serranid.

Telescopus WA GLER 1830 : R.SQUAMAT. (Serpent.) Colubrid.

Telesilla REICHENBACH 1853: Av. APODIF. Trochilid.

Telestes BONAPARTE 1840 : P.-CYPRINIF. (Cyprinoid.) Cyprinid.

Teletrema RIBEIRO 1937: Amph. ANOUR. Leptodactylid.

Telipomis RAFINESQUE 1820 : P.PERCIF. (Percoid.) Centrarchid.

Tellia GERVAIS 1853 : P.-CYPRINODONTIF. Cyprinodontid.

Telmatherina BOULENGER 1897 : P.-MUGILIF. Atherinid.

Telmatobius WIEGMANN 1835 : Amph. ANOUR. Leptodactylid.

Telmatobufo SCHMIDT 1952: Amph. ANOUR. Leptodactylid.

Telmatochromis BOULENGER 1898: P.-PERCIF. (Percoid.) Cichlid.

Telmatodytes CABANIS 1851 : syn. de Cistothorus CABANIS 1851.

Telophonus SWAINSON 1832: Av. PASSERIF. (Acromyod.) Vangid.

Telophorus SWAINSON 1832: Av. PASSERIF. (Acromyod.) Laniid.
Temeculina COCKERELL $1909: P$. CYPRINIF. (Cyprinoid.) Cyprinid.

Temenuchus CABANIS 1851: Av. PASSERIF. (Acromyod.) Sturnid. (Sturnin.).

Temera GRAY 1831 : P.-TORPEDINIF. Torpedinid.

Temnistia RICHARDSON 1836 : P.PERCIF. (Cottoid.) Cottid.

Temnocara BURKE 1930 : P.-PERCIF. (Cottoid.) Cyclopterid.

Temnodon SCHINZ 1822 : P.-PERCIF. (Percoid.) Carangid.

Temnotrogon BONAPARTE 1854 : Av. TROGONIF. Trogonid.

Temnurus LESSON 1831 : Av. PASSERIF. (Acromyod.) Corvid.

Tenes THOMAS 1909 : s./g. de Sciurus LINNE 1758.

Tengujei ISHIYAMA $1958:$ s./g. de Raja LINNE 1758.

Tenrec de LACEPEDE 1799: M.INSECTIV. Tenrecid. (Tenrecin.).

Tentoriceps WHITLEY 1948: P.PERCIF. (Trichiuroid.) Trichiurid.

Tenualosa FOWLER 1934 : s./g. de Hilsa REGAN 1917.

Teonoma GRAY 1843 : s./g. de Neotoma $S A Y$ et ORD 1825.

Tephraeops GUNTHER 1858 : P.PERCIF. (Percoid.) Sparid.

Tephraerops REICHENBACH 1852: Av. CORACIADIF. Meropid.

Tephras HARTLAUB 1868 : syn. de Zosterops VIGORS et HORSFIELD 1826.

Tephrinectes G UN THER 1862 : nom. nov. pro Tephritis GUNTHER 1862.

Tephritis GUNTHER 1862: P.PLEURONECTIF. (Pleuronectoid.) Bothid.

Tephrocorys SHARPE 1875: Av. PASSERIF. (Acromyod.) Alaudid.

Tephrodiglossa CASSIN 1864: Av. PASSERIF. (Acromyod.) Coerebid.

Tephrodornis SWAINSON 1832 : Av. PASSERIF. (Acromyod.) Prionopid. 
Tephrophilus MOORE 1934: Av. PASSERIF. (Acromyod.) Tanagrid.

Tephrozosterops STRESEMANN 1931: Av. PASSERIF. (Acromyod.) Zosteropid.

Terathopius LESSON 1830: Av. FALCONIF. Falconid. (Aquilin.).

Teratichthys GIGLIOLI 1882 : P.NOTACANTHIF. Notacanthid.

Teratohyla : Amph. ANOUR. Leptodactylid.

Teratolepis GUNTHER 1870: R.SQUAMAT. (Saur.) Geckonid.

Teratorhombus MacLEAY 1882 : P. - PLEURONECTIF. (Pleuronectoid.) Bothid.

Teratoscincus STRAUCH 1863 : R.SQUAMAT. (Saur.) Geckonid.

Terekia BONAPARTE 1838: Av. CHARADRIIF. Charadriid. (Scolopacin.).

Terenotriccus RIDGWAY 1907:Av. PASSERIF. (Mesomyod.) Tyrannid.

Terenura CABANIS 1859 : Av. PAS SERIF. (Mesomyod.) Formicariid. (Formicariin.).

Teretistris CABANIS 1855: Av. PASSERIF. (Acromyod.) Parulid.

Teretristis BAIRD 1865 : errat. pro Teretistris CABANIS 1855.

Teretulus RAFINESQUE 1820 : P.CYPRINIF. (Cyprinoid.) Catostomid.

Terpsiphone GLOGER 1827: Av. PASSERIF. (Acromyod.) Muscicapid.

Terrapene MERRIAM 1820: R.TESTUDIN. (Thecoph.) Emydid.

Terreornis : Av. PASSERIF. (Acromyod.) Fringillid.

Terricolumba HACHISUKA 1931: Av. COLUMBIF. Columbid. (Columbin.).

Terrificus LAURENTI 1768: syn. de Crotalus LINNE 1758.

Tersina VIEILLOT 1819: Av. PASSERIF. (Acromyod.) Tanagrid.

Tesia HODGSON 1837 : Av. PASSERIF. (Acromyod.) Sylviid.

Testudo LINNE 1758: R.-TESTUDIN. (Thecoph.) Testudinid.
Tethysia MATHEWS 1933: Av. PROCELLARIIF. Hydrobatid.

Tetrabrachium GUNTHER 1880 : Cyclost. PETROMYZONIF. Tetrabrachiid.

Tetrabranchus BLEEKER 1851: P.-SYMBRANCHIF. Symbranchid.

Tetracentrum MACLEAY 1883 : syn. de Negambassis WHITLEY 1935.

Tetraceros BROOKES 1827: syn. de Tetracervus LEACH 1825.

Tetracervus LEACH 1825 : M.-PARAXON. Bovid. (Tragelaphin.).

Tetrachaetodon WEBER et de BEAUFORT 1936 : P.-PERCIF. (Percoid.) Chaetodontid.

Tetradactylus MERREM 1820 : R.SQUAMAT. (Saur.) Gerrhosaurid.

Tetradrachmum CANTOR 1850: P.-PERCIF. (Labroid.) Pomacentrid.

Tetraenura REICHENBACH 1850 : Av. PASSERIF. (Acromyod.) Ploceid. (Viduin.).

Tetragonops JARDINE 1855: Av. PICIF. Capitonid.

Tetragonopterus CUVIER 1817 : P.-CYPRINIF. (Characoid.) Characid.

Tetragonoptrus WALBAUM 1792 : P.-PERCIF. (Percoid.) Chaetodontid.

Tetragonopyga BANNERMAN 1932: Av. APODIF. Apodid.

Tetragonurus RISSO 1810 : P.-PERCIF. (Stromateoid.) Tetragonurid.

Tetralepis BOETTEGER 1892 : R.SQUAMAT. (Serpent.) Colubrid.

Tetramerodon RHOADS 1894 : syn. de Microtus SCHRANK 1798.

Tetranematichthys BLEEKER 1858: P.-CYPRINIF. (Siluroid.) Doradid.

Tetranesodon WEBER 1913: P.CYPRINIF. (Siluroid.) Bagrid.

Tetrao LINNE 1758: Av. PHASIANIF. Tetraonid.

Tetraodon LINNE 1758: P.-TETRAODONTIF. (Tetraodontoid.) Tetraodontid. 
Tetraogallus GRAY 1832 : Av. PHASIANIF. Phasianid. (Phasianin.).

Tetraophasis ELLIOT 1871: Av. PHASIANIF. Phasianid. (Phasianin.).

Tetrapleurodon CREASER et HUBBS 1922 : syn. de Lampetra OKEN 1816.

Tetraprion STEJNEGER 1891 : Amph. ANOUR. Hylid.

Tetrapteryx THUNBERG 1819 : Av. RALLIF. Gruid.

Tetrapturus RAFINESQUE 1810 : P.-PERCIF. (Scombroid.) Istiophorid.

Tetraroge GUNTHER 1860 : P.PERCIF. (Scorpaenoid.) Scorpaenid.

Tetrastes KEYSERLING et BLASIUS 1840: Av. PHASIANIF. Tetraonid.

Tetrax LEACH 1816: Av. RALLIF. Otidid.

Tetrodon LINNE 1766: vide Tetraodon LINNE 1758.

Tetronarcine TANAKA 1908: P.TORPEDINIF. Torpedinid.

Tetronarce GILL 1862: s./g. de Torpedo HOUTTNYN 1764.

Tetrosomus SWAINSON 1839 : syn. de Rhinesomus SWAINSON 1839.

Teuthis LINNE 1766 : syn. de Siganus FORSKALL 1775 si valide ou de Amphacanthus SCHNEIDER 1801.

Tewara GRIFFIN 1933 : P.-PERCIF. (Trachinoid.) Pinguipedid.

Textor TEMMINCK 1827: Av. PASSERIF. (Acromyod.) Ploceid. (Plocein.).

Thaerodontis Mc CLELLAND 1845 : P. - ANGUILLIF. (Anguilloid.) Muraenid.

Thaigobiella SMITH 1931 : P.-PERCIF. (Gobioid.) Gobiid.

Thalacomys BLYTH 1840 : syn. de Macrotis REID 1837.

Thalarctos GRAY 1825 : M.-FISSIP. Ursid.

Thalassaea KAUP 1829 : Av. PROCELLARIIF. Procellariid.

Thalassarche REICHENBACH
1852 : Av. PROCELLARIF. Diomedeid.

Thalassarctos GRAY 1825 : syn. de Thalarctos GRAY 1825.

Thalasseus BOIE 1822: Av. LARIF. Larid. (Sternin.).

Thalassiarchus KOBELT 1896: syn. de Thalarctos GRAY 1825.

Thalassidroma VIGORS 1825 : syn. d'Hydrobates TEMMINCK 1826.

Thalassinus MOREAU : syn. de Prionace CANTOR 1850.

Thalassornis EYTON 1838: Av. ANATIF. Anatid. (Anatin.).

Thalassoaëtus AGASSIZ 1845: Av. FALCONIF. Falconid. (Aquilin.).

Thalassochelys FITZINGER 1835 : R.-TESTUDIN. (Thecoph.) Chelonid.

Thalassogeron RIDGWAY 1884 : Av. PROCELLARIF. Diomedeid.

Thalassogobius HERRE 1953 : P.PERCIF. (Gobioid.) Gobiid.

Thalassoklephtes GISTEL 1848 : P. - GALEIF. (Carcharhinoid.) Scyliorhinid.

Thalassophina SMITH 1926: R.SQUAMAT. (Serpent.) Hydrophiid.

Thalassophis SCHMIDT 1852 : R.SQUAMAT. (Serpent.) Hydrophiid.

Thalassophryne GUNTHER 1861: P.PERCIF. (Batrachoid.) Batrachoidid.

Thalassorhinus VALENCIENNES 1839: syn. de Prionace CANTOR 1850.

Thalassosteus JORDAN, EVERMANN et TANAKA 1927: P.BELONIF. (Belonoid.) Belonid.

Thaleichthys GIRARD 1858 : P.CLUPEIF. (Salmonoid.) Osmerid.

Thalerophis OLIVER 1947 : syn. de Leptophis BELL 1825.

Thalliurus SWAINSON 1839: P.PERCIF. (Labroid.) Labrid.

Thallomys THOMAS 1920: M.-RODENT. (Myomorph.) Murid. (Murin.).

Thallomyscus THOMAS 1926 : syn. d'Oryzomys BAIRD 1857. 
Thalpomys THOMAS 1916: s./g. d'Akodon MEYEN 1833.

Thalurania GOULD 1848 : Av. APODIF. Trochilid.

Thamnistes SCLATER 1860: Av. PASSERIF. (Mesomyod.) Formicariid. (Thamnophilin.).

Thamnobia SWAINSON 1832: Av. PASSERIF. (Acromyod.) Timaliid.

Thamnocharis SCLATER 1870 : Av. PASSERIF. (Mesomyod.) Formicariid. (Grallarin.).

Thamnodynastes WAGLER 1830 : R.-SQUAMAT. (Serpent.) Boigid.

Thamnolaea CABANIS 1851: Av. PASSERIF. (Acromyod.) Turdid.

Thamnomanes CABANIS 1847 : Av. PASSERIF. (Mesomyod.) Formicariid. (Thamnophilin.).

Thamnomys THOMAS 1907: M.RODENT. (Myomorph.) Murid. (Murin.).

Thamnophilus VIEILLOT 1816 : Av. PASSERIF. (Mesomyod.) Formicariid. (Thamnophilin.).

Thamnophis FITZINGER 1843 : R.SQUAMAT. (Serpent.) Colubrid.

Thamnornis MILNE-EDWARDS 1882: Av. PASSERIF. (Acromyod.) Sylviid.

Thanatophis ARANGO 1889: R.SQUAMAT. (Serpent.) Crotalid.

Thaocervus POCOCK 1943: s./g. de Cervus LINNE 1758.

Thaptomys THOMAS 1916: s./g. d'Akodon MEYEN 1833.

Tharbacus SMITH 1952 : P.-PERCIF. (Batrachoid.) Batrachoidid.

Tharrhaleus KAUP 1829 : Av. PASSERIF. (Acromyod.) Prunellid.

Thaumastomias ALCOCK 1890 : P.CLUPEIF. (Stomiatoid.) Malacosteid.

Thaumastura BONAPARTE 1849 : Av, APODIF. Trochilid.

Thaumatibis ELLIOT 1877: Av. ARDEIF. Plataleid. (Threskiornithin.).

Thaumatichthys SMITH et RADCLIFFE 1912 : P.-LOPHIIF. (Ceratoid.) Ceratiid.
Thayeria EIGENMANN 1909: P.CYPRINIF. (Characoid.) Cha. racid.

Thecadactylus $O K E N$ 1817: R.SQUAMAT. (Saur.) Geckonid.

Thecapteryx FOWLER 1948: P.TETRAODONTIF. (Tetraodontoid.) Tetraodontid.

Thecopsenes FOWLER 1944: P.PERCIF. (Stromateoid.) Stromateid.

Thecopterus SMITH 1907 : P.-PERCIF. (Cottoid.) Cottid.

Thectocercus RIDGWAY 1912 : Av. PSITTACIF. Psittacid.

Thecurus LYON 1907: R.-RODENT. (Hystricomorph.) Hystricid.

Thelotornis SMITH 1849 : R.-SQUAMAT. (Serpent.) Boigid.

Theragra LUCAS 1898 : P.-GADIF. Gadid.

Therauthropus BROOKES 1828 : syn. de Pan OKEN 1816.

Therapaina KAUP 1860 : P.-PERCIF. (Percoid.) Chaetodontid.

Therapon (CUVIER) CLOQUET 1819 : P. - PERCIF. (Percoid.) Serranid.

Theraps GUNTHER 1862 : P.-PERCIF. (Labroid.) Pomacentrid.

Theratopius LESSON 1836: Av. FALCONIF. Falconid. (Aquilin.).

Thereiceryx BLANFORD $1894: A v$. PICIF. Capitonid.

Theristicus WAGLER 1832:Av. ARDEIF. Plataleid. (Threskiornithin.).

Therobromus LUCAS 1898: syn. de Bathylagus GUNTHER 1878.

Theropithecus GEOFFROY-ST-HILAIRE 1843 : M.-PRIMAT. (Simioid.) Cercopithecid. (Cercopithecin.).

Thetomys THOMAS $1910: s . / g$. de Pseudomys GRAY 1832.

Thinocorus ESCHSCHOLTZ 1829 : Av. CHARADRIIF. Thinocorythide.

Thinocorys WAGLER 1830 : emend. pro Thinocorus ESCHSCHOLTZ 1829.

Thinornis GRAY 1845: Av. CHARADRIIF. Charadriid. (Charadriin.). 
Thlypopsis CABANIS 1851: Av. PASSERIF. (Acromyod.) Tanagrid.

Thoburnia JORDAN et SNYDER 1917 : P. - CYPRINIF. (Cyprinoid.) Catostomid.

Tholichthys GUNTHER 1868: P.PERCIF. (Percoid.) Chaetodontid.

Thomasomys COUES 1884 : M.-RODENT. (Myomorph.) Cricetid. (Cricetin. ou Hesperomyin.).

Thomasophantes SCLATER 1925: s.ig. de Ploceus CUVIER 1817.

Thomomys WIED 1839: M.-RODENT. (Myomorph.) Geomyid.

Thoopterus MATSCHIE 1899 : syn. de Cynopterus CUVIER 1824.

Thoracatherina FOWLER 1941: P.-MUGILIF. Atherinid.

Thoracocharax FOWLER 1907 : P.CYPRINIF. (Characoid.) Characid.

Thorichthys MEEK 1904 : P.-PERCIF. (Percoid.) Cichlid.

Thorius COPE 1869 : Amph. UROD. Plethodontid.

Thoropa COPE 1865: Amph. ANOUR. Leptodactylid.

Thorophos FRASER-BRUNNER 1932 : P. - CLUPEIF. (Stomiatoid.) Gonostomatid.

Thos OKEN 1816: $s . / q$. de Canis LINNE 1758.

Thous SMITH 1839 : syn. de Canis LINNE 1758.

Thrasaëtos GRAY 1837: syn. de Harpia VIEILLOT 1816.

Thrasops HALLOWELL 1857 : R.SQUAMAT. (Serpent.) Boigid.

Thraupis BOIE 1826: Av. PASSERIF. (Acromyod.) Tanagrid.

Threnetes GOULD 1852: Av. APODIF. Trochilid.

Threpterius RICHARDSON 1850 : P.-PERCIF. (Trachinoid.) Nototheniid.

Threptria REICHENBACH 1854: Av. APODIF. Trochilid.

Threskiornis GRAY 1842: Av. ARDEIF. Plataleid. (Threskiornithin.).

Thricomys TROUESSART 1881: syn. de Cercomys CUVIER 1829.
Thrinacodus GUNTHER 1879 : M.RODENT. (Caviomorph.) Echimyid. (Dactylomyin.).

Thriothores LESSON 1840 : syn. de Thryothorus VIEILLOT 1816.

Thripadectes SCLATER 1862: Av. PASSERIF. (Mesomyod.) Furnariid.

Thripias CABANIS et HEINE 1863 : Av. PICIF. Picid. (Picin.).

Thriponax CABANIS et HEINE 1863: Av. PICIF. Picid. (Picin.).

Thripophaga CABANIS 1847: Av. PASSERIF. (Mesomyod.) Furnariid.

Thrissa RAFINESQUE 1815 : emend. pro Clupanodon de LACEPEDE 1803.

Thrissa CUVIER 1817 : vide Thryssa CUVIER 1829.

Thrissina JORDAN et SEALE 1925: P.-CLUPEIF. (Clupeoid.) Engraulid. ou Clupeid.

Thrissocharax MYERS 1926: syn. d'Hemigrammocharax PELLEGRIN 1923.

Thrissochromis FOWLER 1941: P.-PERCIF. (Labroid.) Pomacentrid.

Thrissocles JORDAN et EVERMANN 1917: P.-CLUPEIF. (Clupeoid.) Dorosomid.

Thrissomimus JORDAN et EVERMANN 1911 : P.-CLUPEIF. (Salmonoid.) Salmonid.

Thryomanes SCLATER 1862: Av. PASSERIF. (Acromyod.) Trogiodytid.

Tryonomys FITZINGER 1867 : M.RODENT. (Caviomorph.) Echimyid. (Thryonomyin.).

Thryophilus BAIRD 1854: syn. de Thryothorus VIEILLOT 1816.

Thryospiza OBERHOLSER 1917 : Av. PASSERIF. (Acromyod.) Fringillid.

Thryothorus VIEILLOT 1816: Av. PASSERIF. (Acromyod.) Troglodytid.

Thryssa CUVIER 1829: P.-CLUPEIF. (Clupeoid.) Engraulid.

Thunnus SOUTH 1845 : P.-PERCIF. (Scombroid.) Thunnid. 
Thyellodroma KURODA $1954: s . / g$. de Puffinus BRISSON 1760.

Thylacinus TEMMINCK 1824: M.MARSUP. Dasyurid. (Phascogalin.).

Thylacis ILLIGER 1811: syn. d'Isoodon GEOFFROY-ST-HILAIRE 1817.

Thylacomys OWEN 1838: syn. de Macrotis REID 1837.

Thylacomys WAITE 1898 : syn. de Notomys LESSON 1842.

Thylacynus TEMMINCK 1827 : errat. pro Thylacinus TEMMINCK 1824.

Thyliphaps MURPHY 1924: Av. COLUMBIF. Columbid. (Columbin.).

Thylogale GRAY 1837: M.-MARSUP. Macropodid. (Macropodin.).

Thymalloides BERG 1908 : P.-CLUPEIF. (Salmonoid.) Thymallid.

Thymallus CUVIER 1829 : P.-CLUPEIF. (Salmonoid.) Thymallid.

Thynnichthyina FOWLER 1938: s./g. de Thynnichthys BLEEKER 1860 .

Thvnnichthys BLEEKER 1860 : P.CYPRINIF. (Cyprinoid.) Cyprinid.

Thynnus CUVIER 1817: préemploy., syn. de Thunnus SOUTH 1845.

Thyreoconger WADE 1946 : P.-ANGUILLIF. (Congroid.) Congrid.

Thyreorhina PETERS 1871: syn. d'Hipposideros GRAY 1831.

Thyrina JORDAN et CULVER 1895: syn. d'Atherthyrina FOWLER 1958.

Thyrinops HUBBS 1918 : P.-MUGILIF. Atherinid.

Thyris GOODE 1881 : P.-PLEURONECTIF. (Pleuronectoid.) Bothid.

Thyriscus GILBERT et BURKE 1912: P.-PERCIF. (Cottoid.) Cottid.

Thyroptera SPIX 1824 : M.-CHIROPT. (Microchiropt.) Thyropterid.

Thyrorhina SCLATER et SALVIN 1869: Av. RALLIF. Rallid.
Thyrsites LESSON 1831: P.-PEF CIF. (Trichiuroid.) Gempylià.

Thyrsitoides FOWLER 1929: P.PERCIF. (Trichiuroid.) Gempylid.

Thyrsitops GILL 1862 : P.-PERCIF. (Trichiuroid.) Gempylid.

Thyrsoidea KAUP 1856: P.-ANGUILLIF. (Anguilloid.) Muraenid.

Thysanactis REGAN et TREWAVAS 1930: P.-CLUPEIF. (Stomiatoid.) Stomiatid.

Thysanichthys JORDAN et STARKS 1904 : P.-PERCIF. (Scorpaenoid.) Scorpaenid.

Thysanocara REGAN 1906 : P.-CYPRINIF. (Siluroid.) Loricariid.

Thysanocheilus KNER 1864: P.PERCIF. (Labroid.) Labrid.

Thysanophrys JORDAN et EVER$M A N N 1898$ : P.-PERCIF. (Scorpaenoid.) Platycephalid.

Thysanopsetta GUNTHER 1880 : P. - PLEURONECTIF. (Pleuronectoid.) Bothid.

Tiaporus COPE 1892 : syn. d'Ameiva MEYER 1795.

Tiaris SWAINSON 1827: Av. PASSERIF. (Acromyod.) Fringillid.

Tiaroga GIRARD 1856 : P.-CYPRINIF. (Cyprinoid.) Cyprinid.

Tibetholagus ARGYROPOULO et PIDOPLICHKA 1939: syn. d'Ochotona LINK 1795.

Tichodroma ILLIGER 1811: Av. PASSERIF. (Acromyod.) Certhiid.

Tifia JORDAN 1922 : P.-PERCIF. (Percoid.) Chaetodontid.

Tiga KAUP 1836 : Av. PICIF. Picid. (Picin.).

Tigoma GIRARD 1856 : P.-CYPRINIF. (Cyprinoid.) Cyprinid.

Tigrigobius FOWLER 1931 : s./g. de Gobiosoma GIRARD 1858.

Tigriornis SHARPE 1895: Av. ARDEIF. Ardeid.

Tigris OKEN 1816 : s./g. de Panthera OKEN 1816.

Tigris GRAY 1843 : syn. de Tigris OKEN 1816.

Tigrisoma SWAINSON 1827: Av. ARDEIF. Ardeid. 
Tijuca LESSON 1829: Av. PASSERIF. (Mesomyod.) Cotingid.

Tilapia SMITH 1840 : P.-PERCIF. (Percoid.) Cichlid.

Tilesia SWAINSON 1838: P.-GADIF. Gadid.

Tilesina SCHMIDT 1905: P.-PERCIF. (Cottoid.) Agonid.

Tiliqua GRAY 1825 : syn. de Cyclodus WAGLER 1828.

Tilmatura REICHENBACH 1854: Av. APODIF. Trochilid.

Tilodon THOMINOT 1881 : P.-PERCIF. (Percoid.) Chaetodontid.

Tilurella ROULE 1911 : P.-PERCIF (Ophidioid.) Brotulid.

Tiluropsis ROULE 1911: P.-PERCIF. (Ophidioid.) Brotulid.

Tilurus KOELLIKER 1853: P.-PERCIF. (Ophidioid.) Brotulid.

Timalia HORSFIELD 1821: Av. PASSERIF. (Acromyod.) Timaliid.

Timalides BLYTH 1895: Av. PASSERIF. (Acromyod.) Timaliid.

Timelia SUNDEVALL 1872: emend. pro Timalia HORSFIELD 1821.

Timeliopsis SALVADORI 1875 : Av. PASSERIF. (Acromyod.) Timaliid.

Timolia MULSANT 1875: Av. APODIF. Trochilid.

Tinamotis VIGORS 1837: Av. TINAMIF. Tinamid.

Tinamus HERRMANN 1783: Av. TINAMIF. Tinamid.

Tinca CUVIER 1817 : P.-CYPRINIF, (Cyprinoid.) Cyprinid.

Tinnunculus LINNE 1766 : syn. de Falco LINNE 1758.

Tirica BONAPARTE 1854: Av. PSITTACIF. Psittacid.

Tiricoris WHITLEY 1955 : P.-PERCIF. (Labroid.) Labrid.

Tirodon HAY 1882 : P.-CYPRINIF, (Cyprinoid.) Cyprinid.

Tirus RAFINESQUE 1810 : P.-CLUPEIF. (Myctophoid.) Synodid.

Tisa CLARK 1907: Av. PASSERIF. (Acromyod.) Fringillid.

Tityra VIEILLOT 1816: Av. PASSERIF. (Mesomyod.) Cotingid.

Tlaloc ALVAREZ et CARRANZA
1951 : P.-CYPRINODONTIF. Cyprinodontid.

Tobinia WHITLEY 1933 : P.-PERCIF. (Balistoid.) Balistid.

Toccus STRICKLAND 1841: emend. pro Tockus LESSON 1830.

Tocichthys UBBS 1918 : P.-PERCIF. (Labroid.) Embiotocid.

Tockus LESSON 1830: Av. CORACIADIF. Bucerotid.

Todalcyon MATHEWS : syn. d'Alcedo LINNE 1758.

Todarna REICHENBACH 1852 : errat. pro Tadorna OKEN 1817.

Todarus GRASSI et CALANDRUCCIO 1896 : P.-ANGUILLIF. (Congroid.) Congrid.

Todirhamphus (LESSON) VOIGT 1831: Av. CORACIADIF. Alcedinid.

Todirostrum LESSON 1831: Av. PASSERIF. (Mesomyod.) Tyrannid. (Platyrhinchin.).

Todopsis BONAPARTE 1854: Av. PASSERIF. (Acromyod.) Muscicapid.

Todus BRISSON 1760: Av, CORACIADIF. Todid.

Tokudamys JOHNSON 1946: M.RODENT. (Myomorph.) Murid. (Murin.).

Toledia RIBEIRO 1915: P.-PERCIF. (Stromateoid.) Stromateid.

Tolmarchus RIDGWAY 1907: Av. PASSERIF. (Mesomyod.) Tyrannid.

Tolmomyias HELLMAYR 1927: Av. PASSERIF. (Mesomyod.) Tyrannid.

Toluca KENNICOTT 1859 : syn. de Conopsis GUNTHER 1858.

Tolypeutes ILLIGER 1811 : M.EDENT. (Xenarthr.) Dasypodid. (Dasypodin.).

Tometes CUVIER et VALENCIENNES 1849 : P.-CYPRINIF. (Characoid.) Characid.

Tomeutes THOMAS 1915 : syn. de Callosciurus GRAY 1867.

Tomicodon BRISOUT de BARNEVILLE 1846 : P.-PERCIF. (Gobiesoxoid.) Gobiesocid.

Tomistoma MULLER 1846 : R.-LORICAT. Gavialid. 
Tomiyamichthys SMITH 1956 : P.PERCIF. (Gobioid.) Gobiid.

Tomocichla REGAN 1908 : P.-PERCIF. (Percoid.) Cichlid.

Tomodactylus GUNTHER 1900 : Amph. ANOUR. Bufonid.

Tomodon DUMERIL 1853: R.SQUAMAT. (Serpent.) Boigid.

Tomopeas MILLER 1900 : M.-CHIROPT. (Microchiropt.) Vespertilionid. (Tomopeatin.).

Tomopterna DUMERIL et BIBRON 1840: s./g. de Rana LINNE 1758.

Tomuropeltis : $\quad$ R. $-S Q U A M A T$. (Saur.) Amphisbaenid.

Tonatia GRAY 1827 : M.-CHIROPT. (Microchiropt.) Phyllostomatid. (Phyllostomatin.).

Topaza GRAY 1840 : Av. APODIF. Trochilid.

Tor GRAY 1834: P.-CYPRINIF. (Cyprinoid.) Cyprinid.

Torafugu ABE 1954: P.-TETRAODONTIF. (Tetraodontoid.) Tetraodontid.

Torgos KAUP 1828: Av. FALCONIF. Falconid. (Aegypiin.).

Torictus PARR 1951 : P.-CLUPEIF. (Alepocephaloid.) Alepocephalid.

Torpedo HOUTTNYN 1764: P.TORPEDINIF. Torpedinid.

Torquigener WHITLEY 1930 : P.TETRAODONTIF. (Tetraodontoid.) Tetraodontid.

Torrentaria JORDAN et EVERMANN 1896 : P.-PERCIF. (Percoid.) Etheostomid.

Torreornis BARBOUR et PETERS 1927: Av. PASSERIF. (Acromyod.) Fringillid.

Torresia CASTELNAU 1875 : P.PERCIF. (Labroid.) Labrid.

Tortix OPPEL: syn. de Ilysia (HEMPRICH) FITZINGER 1826

Tosana SMITH et POPE 1906 : P.PERCIF. (Percoid.) Plesiopid.

Toshia WHITLEY 1933 : P.-RAJIF. (Dasyatoid.) Dasyatid.

Totanus MOEHRING 1758: Av. CHARADRIIF. Charadriid. (Scolopacin.).

Touit GRAY 1855 : Av. PSITTACIF. Psittacid.
Touraco de LACEPEDE 1799 : Av. CUCULLIF. Musophagid.

Toxabramis GUNTHER 1873 : P.CYPRINIF. (Cyprinoid.) Cyprinid.

Toxicocalamus BOULENGER 1896 : syn. d'Ultrocalamus STERNFELD 1913.

Toxorhamphus STRESEMANN 1914: Av. PASSERIF. (Acromyod.) Meliphagid.

Toxostoma WAGLER 1831: Av. PASSERIF. (Acromyod.) Mimid.

Toxotes CUVIER et CLOQUET 1816 : P.-PERCIF. (Percoid.) Toxotid.

Toxoteuches CABANIS et HEINE 1860 : Av. APODIF. Trochilid.

Toxus EIGENMANN 1904: P.-CYPRINODONTIF. Poeciliid.

Tracheligone MATHEWS 1925: Av. PASSERIF. (Acromyod.) Muscicapid.

Trachelocele ELLERMAN et MORRISON-SCOTT 1951: s./g. de Gazella de BLAINVILLE 1816.

Trachelochismus BRISOUT de BARNEVILLE 1846 : P.-PERCIF. (Gobiesoxoid.) Gobiesocid.

Trachelocirrus DOUMET 1863 : P.PERCIF. (Stromateoid.) Stromateid.

Tracheloptychus PETERS 1854 : R.-SQUAMAT. (Saur.) Gerrhosaurid.

Trachelyopterichthys BLEEKER 1862: P.-CYPRINIF. (Siluroid.) Doradid.

Trachelyopterus CUVIER et VALENCIENNES 1840 : P.-CYPRINIF. (Siluroid.) Doradid.

Trachemys AGASSIZ 1857 : R.-TESTUDIN. (Thecoph.) Emydid.

Trachicephalus SWAINSON 1839 : P.-PERCIF. (Scorpaenoid.) Synancejid.

Trachichthodes GILCHRIST 1903: P.-BERYCIF. Trachichthyid.

Trachichthys SHAW 1799 : P.-BERYCIF. Trachichthyid.

Trachinocephalus GILL 1861: P.CLUPEIF. (Myctophoid.) Synodid. 
Trachinoides BORODIN 1934 : syn. de Merluccius RAFINESQUE 1810.

Trachinoides : P.-PERCIF. (Trachinoid.) Trachinid.

Trachinops GUNTHER 1861: P.PERCIF. (Percoid.) Plesiopid.

Trachinostomias PARR 1927 : P.CLUPEIF. (Stomiatoid.) Stomiatid.

Trachinotus de LACEPEDE 1802: P.-PERCIF. (Percoid.) Carangid.

Trachinus LINNE 1758: P.-PERCIF. (Trachinoid.) Trachinid.

Trachipterus GOUAN 1770 : P.LAMPRIDIF. Trachipterid.

Trachischium GUNTHER 1858 : R.SQUAMAT. (Serpent.) Colubrid.

Trachonurus GUNTHER 1887 : P.GADIF. Macrourid.

Trachops GRAY 1847: M.-CHIROPT. (Microchiropt.) Phyllostomatid. (Phyllostomatin.).

Trachurops GILL 1862: P.-PERCIF. (Percoid.) Carangid.

Trachurus de LACEPEDE 1802: P.-PERCIF. (Percoid.) Carangid.

Trachyberyx ROULE 1929 : P.-BE$R Y C I F$. Trachyberycid.

Trachyboa PETERS 1860: R.SQUAMAT. (Serpent.) Boid.

Trachybrama BLEEKER 1863 : P. CYPRINIF. (Cyprinoid.) Cyprinid.

Trachycephalus DE VIS 1884 : P.PERCIF. (Scorpaenoid.) Scorpaenid.

Trachycephalus TSCHUDI 1838 : Amph. ANOUR. Hylid.

Trachycomus CABANIS 1851: Av. PASSERIF. (Acromyod.) Pycnonotid. ou Parid.

Trachycorystes BLEEKER 1858 : P.-CYPRINIF. (Siluroid.) Doradid.

Trachydermus AGASSIZ 1845 : P.PERCIF. (Cottoid.) Cottid.

Trachydoras EIGENMANN 1925: P.-CYPRINIF. (Siluroid.) Doradid.

Trachyglanis BOULENGER 1902 :
P.-CYPRINIF. (Siluroid.) Bagrid.

Trachylaemus REICHENOW 1891 : Av. PICIF. Capitonid.

Trachynotus BORODIN 1934: errat. pro Trachinotus de LACEPEDE 1802.

Trachyophis WERNER 1926: R.SQUAMAT. (Serpent.) Colubrid.

Trachyops GRAY 1847: syn. de Trachops GRAY 1847.

Trachyphonus RANZANI 1821: Av. PICIF. Capitonid.

Trachypithecus REICHENBACH 1862 : syn. de Presbytis ESCHSCHOLTZ 1821.

Trachypoma GUNTHER 1859 : P.PERCIF. (Percoid.) Serranid.

Trachynoma GIEBEL 1870 : P.-CYPRINIF. (Siluroid.) Trichomycterid.

Trachypterophrys FRANZ 1911: P.-PLEURONECTIF. (Pleuronectoid.) Bothid.

Trachypterus SCHNEIDER 1801: syn. de Trachipterus GOUAN 1770.

Trachyrhamphus KAUP 1853 : P.PERCIF. (Gobioid.) Gobiid.

Trachyrhamphus : P.-S YNGNATHIF. Syngnathid.

Trachyrhynchus GIORNA 1809 : P.-GADIF. Macrurid.

Trachysaurus GRAY 1826 : syn. de Tíliqua GRAY 1825.

Trachyscorpia GINSBURG 1953 : P.-PERCIF. (Scorpaenoid.) Scorpaenid.

Trachytoma DOUGLAS-OGILBY 1888 : P.-MUGILIF. Mugilid.

Trachysurus RAFINESQUE 1815: P.-CYPRINIF. (Siluroid.) Bagrid.

Tragelaphus de BLAINVILLE 1816: M.-PARAXON. Bovid. (Tragelaphin.).

Tragopan GRAY 1829: Av. PHASIANIF. Phasianid. (Phasianin.).

Tragops WAGLER 1830 : syn. de Dryophis DALMAN 1823.

Tragops HODGSON 1847 : syn. de Gazella de BLAINVILLE 1816.

Tragopsis FITZINGER 1869 : syn. 
de Gazella de BLAINVILLE 1816.

Tragulichthys WHITLEY 1931 : P.TETRAODONTIF. (Tetraodontoid.) Diodontid.

Tragulus PALLAS 1779: M.-PARAXON. Tragulid.

Tragulus BRISSON 1762: syn. de Tragulus PALLAS 1779.

Tragus SCHRANK 1798: syn. de Capra LINNE 1758.

Tralatitius GRAY 1866: syn. de Myotis KAUP 1829.

Tralatitus GERVAIS 1849 : syn, de Myotis KAUP 1829.

Travancoria HORA 1941 : syn. de Homaloptera van HASSELT 1823.

Tregellasia MATHEWS 1912: Av. PASSERIF. (Acromyod.) Muscicapid.

Tremarctos GERVAIS 1855: M.FISSIP. Ursid.

Trematocara BOULENGER 1899 : P.-PERCIF. (Percoid.) Cichlid.

Trematocranus TREWAVAS 1935 : P.-PERCIF. (Percoid.) Cichlid.

Trematomus BOULENGER 1902: P.-PERCIF. (Trachinoid.) Nototheniid.

Trematorhynchus REGAN et TREWAVAS 1933 : P.-LOPHIIF. (Ceratoid.) Himantolophid.

Treron VIEILLOT 1816: Av. COLUMBIF. Columbid. (Treronin.).

Tretanorhinus DUMERIL et BI$B R O N$ 1854: R.-SQUAMAT. (Serpent.) Colubrid.

Tretioscincus COPE 1862：R.SQUAMAT. (Saur.) Tejid.

Trewara GRIFFIN 1933: P.-PERCIF. (Trachinoid.) Pinguipedid.

Triancanthodes BLEEKER 1858: P.-PERCIF. (Balistoid.) Triacanthid.

Triacanthopsis : P.-PERCIF. (Percoid.) Pempherid.

Triacanthurodes FOWLER 1944: P.-PERCIF. (Siganoid.) Siganid.

Triacanthus (CUVIER) OKEN 1817: P.-PERCIF. (Balistoid.) Triacanthid.

Triadulifer FRASER-BRUNNER
1940 : s./g. de Paratriacanthodes FOWLER 1934.

Triaenapogon BLEEKER 1874 : P.PERCIF. (Gobioid.) Gobiid.

Triaenodon MULLER et HENLE 1837: P.-GALEIF. (Carcharhinoid.) Carcharhinid.

Triaenopholis WERNER 1924 : R.SQUAMAT. (Serpent.) Colubrid.

Triaenophorichthys GILL 1859 : P.-PERCIF. (Gobioid.) Gobiid.

Triaenophorus GILL 1858 : syn. de Triaenophorichthys GILL 1859.

Triaenops DOBSON 1871 : M.-CHIROPT. (Microchiropt.) Rhinolophid. (Hipposiderin.).

Triaerus : P.-CLUPEIF. (Stomiatoid.) Gonostomid.

Triakis MULLER et HENLE 1838 : P. -GALEIF. (Carcharhinoid.) Carcharhinid.

Trianectes RAMSAY et OGILBY 1918: syn. de Verconectes WHITLEY 1931.

Triantennatus SCHULTZ 1957 : s./g. de Phrynelox WHITLEY 1931.

Trianthallosothia FOWLER 1943 : P.-PERCIF. (Batrachoid.) Batrachoidid.

Tribolodon SAUVAGE 1883 : P.-CYPRINIF. (Cyprinoid.) Cyprinid.

Tribolonotus DUMERIL et BIBRON 1839: R. -SQUAMAT. (Saur.) Scincid.

Tribolurus : errat. pro Triloburus JORDAN 1882.

Tribonvx DU BUS 1840: Av. RALLIF. Rallid.

Tribranchus PETERS 1844: P.ANGUILLIF. (Anguilloid.) Anguillid.

Tribura HODGSON 1844 : s./g. de Bradypterus SWAINSON 1837.

Triccus CABANIS 1845: Av. PASSERIF. (Mesomyod.) Tyrannid.

Trichaelurus SATUNIN 1905 : syn. de Felis LINNE 1758.

Trichastoma BLYTH 1842: syn. d'Alcippe BLYTH 1854.

Trichechus MULLER 1773 : syn. de Dugong de LACEPEDE 1799.

Trichechus LINNE 1758: M.-SIREN. Trichechid. 
Trichechus LINNE 1766: syn. de Odobenus BRISSON 1762.

Trichidion WALBAUM 1792: P.POLYNEMIF. Polynemid.

Trichiurus LINNE 1758: P.-PERCIF. (Trichiuroid.) Trichiurid.

Trichixos LESSON 1839: Av. PASSERIF. (Acromyod.) Timaliid.

Trichobatrachus BOULENGER 1900 : Amph. ANOUR. Ranid.

Trichobrachirus CHABANAUD 1943 : P.-PLEURONECTIF. (Soleoid.) Soleid.

Trichochirus REGAN et TREWAVAS $1930:$ s./g. de Bathophilus GIGLIOLI 1884.

Trichocichla REICHENOW 1890 : syn. d'Ortygocichla SCLATER 1881.

Trichocottus SOCDATOV et PAVLENKO 1916 : P.-PERCIF. (Cottoid.) Cottid.

Trichocyclus GUNTHER 1870 : P.TETRAODONTIF. (Tetraodontoid.) Diodontid.

Trichodiodon BLEEKER 1865 : P.TETRAODONTIF. (Tetraodontoid.) Diodontid.

Trichodon CUVIER 1829 : P.-PERCIF. (Trachinoid.) Trachinid.

Trichodroma : vide Tichodroma ILLIGER 1811.

Trichogaster SCHNEIDER 1801 : syn. de Colisa CUVIER et VALENCIENNES 1831.

Trichoglossus VIGORS et HORSFIELD 1826: Av. PSITTACIF. Psittacid.

Tricholaema VERREAUX et VERREAUX 1855 : Av. PICIF. Capitonid.

Tricholestes SALVADORI 1874: Av. PASSERIF. (Acromyod.) Pycnonotid. ou Parid.

Tricholimnas SHARPE 1893: Av. RALLIF. Rallid.

Trichomanis HUBRECHT 1891 : syn. d'Arctonyx CUVIER 1825.

Trichometopus CABANIS 1851: syn. de Chibia HODGSON 1837.

Trichomycterus VALENCIENNES 1833: P.-CYPRINIF. (Siluroid.) Trichomycterid.

Trichonotus SCHNEIDER 1801:
P.-PERCIF. (Trachinoid.) Pinguipedid.

Trichonotus RAFINESOUE 1815 : P.-CLUPEIF. (Elopoid.) Elopid.

Trichoparadisea MEYER $1893:$ Av. PASSERIF. (Acromyod.) Paradiseid. (Paradisein.).

Trichopharynx OGILBY 1898: P. PERCIF. (Gobioid.) Gobiid.

Trichophoropsis BONAPARTE 1854: Av. PASSERIF. (Acromyod.) Parid. ou Pycnonotid.

Trichophorus TEMMINCK 1821: Av. PASSERIF. (Acromyod.) Pycnonotid.

Trichopicus BONAPARTE 1854: Av. PICIF. Picid.

Trichopipo CABANIS 1863: Av. PASSERIF. (Mesomyod.) Piprid

Trichonleura KAUP 1858 : P.-PERCIF. (Scorpaenoid.) Aploactid.

Trichopodus de LACEPEDE 1802 : P.-PERCIF. (Anabantoid.) Anabantid.

Trichopsetta GILL 1889 : P.-PLEURONECTIF. (Pleuronectoid.) Bothid.

Trichonsis KNER 1860 : P.-PERCIF. (Anabantoid.) Anabantid.

Trichopterus GRONOV 1854: P.PERCIF. (Percoid.) Cheilodactylid.

Trichopus SHAW 1803: syn. de Trichopodus de LACEPEDE 1802.

Trichosoma SWAINSON 1838 : P.CLUPEIF. (Clupeoid.) Engraulid.

Trichostoma (BLYTH) STRICKLAND 1849: Av. PASSERIF. (Acromyod.) Timaliid.

Trichostomias ZUGMAYER 1911: s./g. de Bathophilus GIGLIOLI 1884.

Trichosurus LESSON 1828: M.MARSUP. Phalangerid. (Phalangerin.).

Trichothraupis CABANIS 1851:Av. PASSERIF. (Acromyod.) Tanagrid.

Trichys GUNTHER 1876: M.-RODENT. (Hystricomorph.) Hystricid.

Triclaria WAGLER 1832: Av. PSITTACIF. Psittacid. 
Triclonostomias REGAN et TREWAVAS 1930: s./g. d'Eustomias VAILLANT 1888.

Tridens EIGENMANN 1889 : P.-CYPRINIF. (Siluroid.) Trichomycterid.

Tridensimilis SCHULTZ 1944 : P.CYPRINIF. (Siluroid.) Trichomycterid.

Tridentiger GILL 1858: P.-PERCIF. (Cottoid.) Cottid.

Tridentopsis MYERS 1925 : P.-CYPRINIF. (Siluroid.) Trichomycterid.

Trifarcius POEY 1860 : P.-CYPRINODONTIF. Poeciliid.

Trifissus JORDAN et SNYDER 1900 : P.-PERCIF. (Gobioid.) Gobiid.

Trigla LINNE 1758: P.-PERCIF. (S̈corpaenoid.) Triglid.

Triglochis MULLER et HENLE 1837 : P.-GALEIF. (Carcharhinoid.) Carcharhinid.

Trigloporus SMITH 1934 : P.-PERCIF. (Scorpaenoid.) Triglid.

Triglops REINHARDT 1832 : P.PERCIF. (Cottoid.) Cottid.

Triglopsis GIRARD 1851: syn. de Myoxocephalus TILESIUS 1811.

Triglyphodon DUMERIL 1853: syn. de Dipsadomorphus FITZINGER 1843.

Trigon: syn. de Dasyatis RUPPELL 1829.

Trigonectes MYERS 1927 : P.-CYPRINODONTIF. Cyprinodontoid.

Trigonocephalus OPPELL 1811: syn. de Lachesis DAUDIN pp. et de Ancistrodon PALISOT pp.

Trigonoceps LESSON 1842: Av. FALCONIF, Falconid. (Aegypiin.).

Trigonodactylus HAAS 1957: R.SQUAMAT. (Saur.) Geckonid.

Trigonolampa REGAN et TREWAVAS 1930 : P.-CLUPEIF. (Stomiotoid.) Melanostomiatid.

Trigonophallus HUBBS 1926 : P.CYPRINODONTIF. Goodeid.

Trigonophis EICHEWALD 1831 : R.-SQUAMAT. (Saur.) Amphisbaenid.
Trilatitus GRAY 1842: syn. de Myotis KAUP 1829.

Triloburus JORDAN 1882 : P.-PERCIF. (Percoid.) Serranid.

Trimegistius : errat. pro Trismegistius JORDAN et SNYDER 1904.

Trimeresurus de LACEPEDE 1804: R.-SQUAMAT. (Serpent.) Crotalid.

Trimerodytes COPE 1895 : syn. de Liparophis PERACCA 1904.

Trimerorhinus SMITH 1847: R.SQUAMAT. (Serpent.) Boigid.

Trimesurus GRAY 1840: syn. de Lachesis DAUDIN 1803.

Trimetopon COPE 1855 : R.-SQUAMAT. (Serpent.) Colubrid.

Trimma JORDAN et SEALE 1906 : P.-PERCIF. (Gobioid.) Eleotrid.

Trimolagus GUREEV 1947: s./g. de Lepus LINNE 1758.

Trimorphodon COPE 1869: R.SQUAMAT. (Serpent.) Boigid.

Trinectes RAFINESQUE 1832 : P.PLEURONECTIF. (Soleoid.) Soleid.

Trinematichthys BLEEKER 1860 : P.-CYPRINIF. (Cyprinoid.) Cyprinid.

Tringa LINNE 1758: Av. CHARADRIIF. Charadriid. (Scolopacin.).

Tringoides BONAPARTE 1827: Av. CHARADRIIF. Charadriid. (Scolopacin.).

Trinodontomys RHOADS 1894 : syn. de Peromyscus GLOGER 1841.

Trinomys THOMAS $1921: s . / g$. de Proechimys ALLEN 1899.

Triodon CUVIER 1829 : P.-TETRAODONTIF. (Tetraodontoid.) Triodontid.

Trionyx GEOFFROY-ST-HILAIRE 1809 : R.-TESTUDIN. (Thecoph.) Trionychid.

Triorchis LEACH 1816: syn. de Buteo de LACEPEDE 1799.

Triorus JORDAN et HUBBS 1925 : syn. de Rhinesomus SWAINSON 1839.

Triplophos BRAUER 1902 : P.-CLUPEIF. (Stomiatoid.) Gonostomid. 
Triplophysa RENDHAL 1933 : P.CYPRINIF. (Cyprinoid.) Cobitid.

Triportheus COPE 1872 : P.-CYPRINIF. (Characoid.) Characid.

Triprion COPE 1866: Amph. ANOUR. Hylid.

Tripsurus SWAINSON 1837: Av. PICIF. Picid.

Tripterodon PLAYFAIR 1866 : P.PERCIF. (Percoid.) Sparid.

Tripteronotus de LACEPEDE 1803: P.-CLUPEIF. (Salmonoid.) Salmonid.

Tripterophycis BOULENGER 1902 : P.-GADIF. Gadid.

Tripterorhinus CABANIS: syn. d'Eugralla LESSON 1842.

Tripterygion RISSO 1826 : P.-PERCIF.(Blennioid.) Clinid.

Trirhinopholis BOULENGER 1892 : R.-SQUAMAT. (Serpent.) Colubrid.

Triscurrichthys WHITLEY 1931 : P.-PERCIF. (Scorpaenoid.) Triglid.

Trimegistius JORDAN et SNYDER 1904: P.-PERCIF. (Cottoid.) Cyclopterid.

Trisopterus RAFINESQUE 1814: P.-GADIF. Gadid.

Trisotropis GILL 1865 : P.-PERCIF. (Percoid.) Serranid.

Tristramella TREWAVAS 1942 : P. PERCIF. (Percoid.) Cichlid.

Triton LINNE 1758: Amph. UROD. Salamandrid.

Trituroides CHANG 1936: Amph. UROD. Salamandrid.

Triturus RAFINESQUE 1815 : syn. de Triton LINNE 1758.

Triurobrycon EIGENMANN 1909 : P.-CYPRINIF. (Characoid.) Characid.

Triurus de LACEPEDE 1800 : P.CLUPEIF. (Alepocephaloid.) Alepocephalid.

Trixiphichthys FRASER-BRUNNER 1940: s./g. de Triacanthus (CUVIER) OKEN 1817.

Trochalopteron HODGSON 1843 : Av. PASSERIF. (Acromyod.) Timaliid.

Trochalopterum AGASSIZ 1846 : emend. pro Trochalopteron HODGSON 1843.

Trochilus LINNE 1758: Av. APODIF. Trochilid.

Trochocercus CABANIS 1850: Av. PASSERIF. (Acromyod.) Muscicapid.

Trochopus GUNTHER 1862 : P.PERCIF. (Labroid.) Labrid.

Trochus KLUK : syn. de Mellisuga BRISSON 1760.

Troglichthys EIGENMANN 1899 : P.-AMBLYOPSIF. Amblyopsid.

Troglodytes GEOFFROY-ST-HILAIRE 1812: syn. de Pan OKEN 1816.

Troglodytes MOEHRING 1758: Av. PASSERIF. (Acromyod.) Troglodytid.

Trogloglanis EIGENMANN 1919 : P.-CYPRINIF. (Siluroid.) Silurid.

Trogon MOEHRING 1758: Av. TROGONIF. Trogonid.

Trogonophis KAUP 1830: R.-SQUAMAT. (Saur.) Amphisbaenid.

Trogonurus BONAPARTE 1854: Av. TROGONIF. Trogonid.

Trogonopterus HEUDE 1898 : M.RODENT. (Sciuromorph.) Sciurid. (Petauristin.).

Trompe GISTEL 1848 : P.-PERCIF. (Percoid.) Toxotid.

Tropheus BOULENGER 1898: P.PERCIF. (Percoid.) Cichlid.

Tropicolobus de ROCHEBRUNE 1887: syn. de Colobus ILLIGER 1811.

Tropicoperdix BLYTH 1859: Av. PHASIANIF. Phasianid. (Phasianin.).

Tropicranus SCLATER 1922: Av. CORACIADIF, Bucerotid.

Tropidechis GUNTHER 1863: R.SQUAMAT. (Serpent.) Elapid.

Tropidichthys BLEEKER 1854 : P.TETRAODONTIF. (Tetraodontoid.) Canthigasterid.

Tropidinius GILL 1868: P.-PERCIF. (Percoid.) Lutjanid.

Tropidoclonion $C O P E$ 1860: R.SQUAMAT. (Serpent.) Colubrid.

Tropidodactylus BOULENGER 1885 : R.-SQUAMAT. (Saur.) Iguanid. 
Tropidodipsas GUNTHER 1858: R.-SQUAMAT. (Serpent.) Colubrid.

Tropidodus GILL 1862 : P.-HETERODONTIF. Heterodontid.

Tropidolepis GRAY 1830 : syn. de Gerrhonotus WIEGMANN 1828.

Tropidonotus KUHL 1826 : syn. de Natrix LAURENTI 1768.

Tropidoperdix : errat. pro Tropicoperdix BLYTH 1859.

Tropidophis COCTEAU et BIBRON 1843: R.-SQUAMAT. (Serpent.) Boid. (Boin.).

Tropidophorus DUMERIL et BIBRON 1839: R.-SQUAMAT. (Saur.) Scincid.

Tropidosaura BOIE 1826: R.-SQUAMAT. (Saur.) Lacertid.

Tropidostethus DOUGLAS-OGILBY 1895 : P.-MUGILIF. Atherinid.

Tropidurus WIED 1824 : R.-SQUAMAT. (Saur.) Iguanid.

Tropidocolotes PETERS 1880 : R.SQUAMAT. (Saur.) Geckonid.

Trudis WHITLEY 1931: P.-PERCIF. (Scorpaenoid.) Platycephalid.

Trulla KAUP 1858: s./g. de Cynoglossus HAMILTON-BUCHANAN 1822.

Trugon GRAY 1849: Av. COLUMBIF. Columbid.

Trupialis MERREM 1826 : syn. d'Icterus BRISSON 1760.

Trupialis BONAPARTE 1850: Av. PASSERIF. (Acromyod.) Icterid. (Sturnellin.).

Trutta GEOFFROY - ST - HILAIRE 1767: syn. de Salmo LINNE 1758.

Trycherodon FORBES 1883 : P.CYPRINIF. (Cyprinoid.) Cyprinid.

Trygon CUVIER 1817 : syn. de Dasyatis RUPPELL 1829.

Trygonobatus de BLAINVILLE 1816: syn. de Urobatis GARMAN 1913.

Trygonoptera MULLER et HENLE 1841: P.-RAJIF. (Dasyatoid.) Dasyatid.

Trigonorhina MULLER et HENLE 1841 : P.-RAJIF. (Rhinobatoid.) Rhinobatid.
Trygonorrhina MULLER et HENLE 1838: syn. de Trygonorhina MULLER et HENLE 1841.

Tryngas GLOGER 1842: syn. de Tringa LINNE 1758.

Tryngites CABANIS 1856: Av. CHARADRIIF. Charadriid. (Scolopacin.).

Trypanocorax KAUP 1854: Av. PASSERIF. (Acromyod.) Corvid.

Trypanurgos FITZINGER 1846 : syn. d'Eudipsas FITZINGER 1843.

Trypauchen CUVIER et VALENCIENNES 1837：P.-PERCIF. (Callionymoid.) Callionymid. (ou Gobiid.).

Trypauchenichthys BLEEKER 1860 : P.-PERCIF. (Callionymaoid.) Callionymid.

Trypauchenophrys FRANZ 1911: P.-PERCIF. (Gobioid.) Gobiid.

Trypauchenopsis VOLTZ 1903 : P.PERCIF. (Callionymoid.) Callionymid.

Tryphomys MILLER 1910 : M.-RODENT. (Myomorph.) Murid. (Murin.).

Trypterodon: vide Tripterodon PLAYFAIR 1866.

Tschagra: vide Tchagra LESSON 1831.

Tschagroides ROBERTS 1922 : vide Tchagroides ROBERTS 1922.

Tscherskia OGNEV 1914 : s./g. de Cricetulus MILNE-EDWARDS 1867.

Tubbia WHITLEY 1943 : P.-PERCIF. (Stromateoid.) Stromateid.

Tuitatus KISHIDA et MORI 1931: syn. d'Eptesicus RAFINESQUE 1820.

Tukugobius HERRE 1927 : P.-PERCIF. (Gobioid.) Eleotrid.

Tulelepis SMITH 1954 : P.-PERCIF. (Percoid.) Serranid.

Tumbezia CHAPMAN 1925: Av. PASSERIF. (Mesomyod.) Tyrannid.

Tupa de BUEN 1954 : s./g. d'Odontesthes EVERMANN et KEN$D A L L 1906$.

Tupaia RAFFLES 1821 : M.-INSECTIV. Tupaid. (Tupain.). 
Tupinambis DAUDIN 1802 : R.SQUAMAT. (Saur.) Tejid.

Turacoena BONAPARTE 1854: Av. COLUMBIF. Columbid. (Columbin.).

Turacus CUVIER 1800 : vide Touraco de LACEPEDE 1799.

Turanomolge : Amph. UROD. Ambystomatid.

Turckichthys : P.-CYPRINODONTIF. Cyprinodontid.

Turdinulus HUME 1878 : Av. PASSERIF. (Acromyod.) Timaliid.

Turdinus BLYTH 1844: Av. PASSERIF. (Acromyod.) Timaliid.

Turdoides CRETZSCHMAR 1826 : Av. PASSERIF. (Acromyod.) Timaliid. (Turdoidin.).

Turdus LINNE 1758: Av. PASSERIF. (Acromyod.) Turdid. (Turdin.).

Turnagra LESSON 1837 : Av. PASSERIF. (Acromyod.) Timaliid.

Turnix BONNATERRE 1790: Av. RALLIF. Turnicid.

Turocapra de BEAUX 1949: syn. de Capra LINNE 1758.

Turrum WHITLEY 1932 : P.-PERCIF. (Percoid.) Carangid.

Tursio WAGLER 1830 : syn. de Lissodelphis GLOGER 1841.

Tursiops GERVAIS 1855: M.-CETAC. (Odontocet.) Delphinid.

Turtur BODDAERT 1783: Av, COLUMBIF. Columbid. (Columbin.).

Turtur SELBY 1835 : syn. de Streptopelia BONAPARTE 1854.

Turturoena BONAPARTE 1854 : Av. COLUMBIF. Columbid. (Columbin.).

Turus HILZHEIMER 1916 : syn, de Capra LINNE 1758.

Twingonia PASCOE 1908 : P.-PERCIF. (Percoid.) Sciaenid.

Tychaedon RICHEMOND 1917 : Av. PASSERIF. (Acromyod.) Turdid.

Tydemania WEBER 1913 : P.-PERCIF. (Balistoid.) Triacanthid.

Tylas HARTLAUB 1862: Av. PASSERIF. (Acromyod.) Pycnonotid. ou Parid.
Tylibyx REICHENBACH 1852: Av. CHARADRIIF. Charadriid. (Charadriin.).

Tylobranchus EIGENMANN 1912 : P.-CYPRINIF. (Characoid.) Characid.

Tylochromis REGAN 1920 : P.-PERCIF. (Percoid.) Cichlid.

Tylognathus HECKEL 1843: P. CYPRINIF. (Cyprinoid.) Cyprinid.

Tylomys PETERS 1866 : M.-RODENT. (Myomorph.) Cricetid. (Cricetin.).

Tylonycteris PETERS 1872: M.CHIROPT. (Microchiropt.) Vespertilionid. (Vespertilionin.).

Tylostoma GERVAIS 1855 : syn. de Phyllostomus de LACEPEDE 1799.

Tylosurus COCCO 1833 : P.-BELONIF. (Belonoid.) Belonid.

Tylotriton ANDERSON 1871 : Amph. UROD. Salamandrid.

Tympanistria REICHENBACH 1853: Av. COLUMBIF. Columbid. (Columbin.).

Tympanocryptis PETERS 1863 : R.-SQUAMAT. (Saur.) Agamid.

Tympanopleura EIGENMANN 1912 : P.-CYPRINIF. (Siluroid.) Doradid.

Tympanuchus GLOGER 1842: Av. PHASIANIF. Tetraonid.

Tyntlastes GUNTHER 1862 : P.PERCIF. (Gobioid.) Gobiid.

Typhlachirus HERDENBERG 1931: P.-PLEURONECTIF. (Soleoid.) Soleid.

Typhlacontias BARBOZA du BOCAGE 1873: R.-SQUAMAT. (Saur.) Scincid.

Typhle AGASSIZ 1846: P.-SYNGNATHIF. Syngnathid.

Typhleleotris PETIT 1933 : P.-PERCIF. (Gobioid.) Eleotrid.

Typhlias HUBBS 1938: syn. de Typhliasima.

Typhliasina WHITLEY 1951 : P.PERCIF. (Ophidioid.) Brotulid.

Typhlichthys GIRARD 1860 : P.$A M B L Y O P S I F$. Amblyopsid.

Typhlobagrus RIBEIRO 1907: P.CYPRINIF. (Siluroid.) Bagrid. 
Typhlobelus MYERS 1944 : P.-CYPRINIF. (Siluroid.) Trichomycterid.

Typhlobranchus SCHNEIDER 1801:P.-SYMBRANCHIF. Symbranchid.

Typhloceratias BARBOUR 1941: P.-LOPHIIF. (Ceratoid.) Ceratiid.

Typhlogarra TREWAVAS 1955 : P.-CYPRINIF. (Cyprinoid.) Cyprinid.

Typhlogeophis GUNTHER 1879 : R.-SQUAMAT. (Serpent.) Colubrid.

Typhlogobius STEINDACHNER 1880: P.-PERCIF. (Gobioid.) Gobiid.

Typhlomolge STEJNEGER 1896 : Amph. UROD. Proteid.

Typhlomys MILNE-EDWARDS 1877: M. - RODENT. (Myomorph.) Platacanthomyid. (Typhlomyin.).

Typhlonarke WAITE 1900 : P.TORPEDINIF. Torpedinid.

Typhlonectes PETERS 1879: Amph. APOD. Caecilitd.

Typhlonus GUNTHER 1878 : P.PERCIF. (Ophidioid.) Brotulid.

Typhlophis FITZINGER 1843: R.SQUAMAT. (Serpent.) Typhlopid.

Typhlops OPPELL 1811 : R.-SQUAMAT. (Serpent.) Typhlopid.

Typhlosaurus WIEGMANN 1838 : R.-SQUAMAT. (Saur.) Anelytropid.

Typhlotes FISCHER 1813 : P.-ANGUILLIF. (Congroid.) Ophichthyid.

Typhlotriton STEJNEGER 1892 : Amph. UROD. Desmognathid.

Typhobagrus: vide Typhlobagrus RIBEIRO 1907.

Typhon REICHENBACH 1852 : Av ARDEIF. Ardeid.

Typomys THOMAS 1911: syn. d'Hybomys THOMAS 1910.

Tyranneutes SCLATER 1881: Av, PASSERIF. (Mesomyod.) Tyrannid.

Tyranniscus CABANIS 1851: Av. PASSERIF. (Acromyod.) Tyrannid. (Spizitornin.).
Tyrannophryne REGAN et TREWAVAS 1932 : P.-LOPHIIF. (Ceratoid.) Himantolophid.

Tyrannopsis RIDGWAY 1907: Av. PASSERIF. (Acromyod.) Tyrannid.

Tyrannula SWAINSON 1827 : syn. de Serpophaga GOULD 1839.

Tyrannulus VIEILLOT 1816: Av. PASSERIF. (Mesomyod.) Tyrannid. (Spizitornin.).

Tyrannus de LACEPEDE 1799 : Av. PASSERIF. (Mesomyod.) Tyrannid. (Tyrannin.).

Tyto BILLBERG 1828: Av. STRIGIF. Strigid. (Tytonin.).

Tyttocharax FOWLER 1913 : P.CYPRINIF. (Characoid.) Characid.

Tzarevscya REUSS 1930 : R.-SQUAMAT. (Serpent.) Viperid.

\section{$\mathbf{U}$}

Uaru HECKEL 1840 : P.-PERCIF. (Percoid.) Cichlid.

Ubidia MILES 1945 : P.-CYPRINIF. (Gymnotoid.) Sternarchid.

Uegitglanis GIANFERRARI 1923 : P.-CYPRINIF. (Siluroid.) Clariid.

Ugaliophis MULLER 1882: vide Ungaliophis MULLER 1880.

Ujhelyiana STRAND 1922 : syn. de Spalax GULDENSTAEDT 1770.

Ulaema JORDAN et EVERMANN 1891 : P.-PERCIF. (Percoid.) Leiognathid.

Ulapiscis WHITLEY 1933 : P.-PERCIF. (Percoid.) Lutjanid.

Ulaula JORDAN et THOMPSON 1911 : P.-PERCIF. (Percoid.) Lutjanid.

Ulca JORDAN et EVERMANN 1896: P.-PERCIF. (Cottoid.) Cottid.

Ulcigobius FOWLER 1918 : P.-PERCIF. (Gobioid.) Gobiid.

Ulcina CRAMER 1896 : P.-PERCIF. (Cottoid.) Agonid.

Ulocentra JORDAN 1878 : P.-PERCIF. (Percoid.) Etheostomid.

Ultimostomias BEEBE 1933 : P.CLUPEIF. (Stomiatoid.) Malacosteid. ou Photostomiatid. 
Ultrocalamus STERNFELD 1913 : R.-SQUAMAT. (Serpent.) Elapid.

Ulua JORDAN et SNYDER 1908 : P.-PERCIF. (Percoid.) Carangid.

Ulvicola GILBERT 1897 : P.-PERCIF. (Blennioid.) Blenniid.

Uma BAIRD 1858 : R.-SQUAMAT. (Saur.) Iguanid.

Umalius HERRE et HERALD 1951: P.-PERCIF. (Ophidioid.) Ophidiid.

Umbla CATESBY 1777: syn. de Salvelinus RICHARDSON 1836.

Umbra GRONOV 1763 : P.-CLUPEIF. (Esoxoid.) Umbrid.

Umbretta RAFINESQUE 1815:Av.

Umbrina CUVIER 1817 : P.-PERCIF. (Percoid.) Sciaenid.

Unagius JORDAN 1919 : P.-ANGUILLIF. (Anguilloid.) Moringuid.

Uncia GRAY 1854: s./g. de Panthera OKEN 1816.

Undecimus WHITLEY 1934 : P.PERCIF. (Percoid.) Lutjanid.

Ungalia GRAY 1842 : R.-SQUAMAT. (Serpent.) Boid.

Ungaliophis MULLER 1880: R.SQUAMAT. (Serpent.) Boid.

Uniantennatus SCHULTZ 1957: s./g. de Lophiocharon WHITLEY 1933.

Unibranchapertura de LACEPEDE 1803 : P.SYMBRANCHIF. Symbranchid.

Unicomys TROUGHTON 1935 : syn. de Melomys THOMAS 1922.

Unipertura DUMERIL 1856 : errat. pro Unibranchapertura de LACEPEDE 1803.

Upeneichthys BLEEKER 1855 : P.PERCID. (Percoid.) Mullid.

Upeneoides BLEEKER 1849: P.PERCIF. (Percoid.) Mullid.

Upeneus CUVIER et VALENCIENNES 1829: P.-PERCIF. (Percoid.) Mullid.

Uperodon DUMERIL et BIBRON 1841 : Amph. ANOUR. Microhylid.

Uperoleia GRAY 1841: Amph. ANOUR. Leptodactylid,
Upsilodus RIBEIRO 1925 : P.-CYPRINIF. (Siluroid.) Loricariid.

Upsilognathus : P.-PERCIF. (Trachinoid.) Opisthognathid.

Upucerthia GEOFFROY-ST-HILAIRE 1832: Av. PASSERIF. (Mesomyod.) Furnariid.

Upupa LINNE 1758: Av. CORACIADIF. Upupid.

Uradia : P.-PERCIF. (Scorpaenoid.) Triglid.

Uraeginthus CABANIS 1851: Av. PASSERIF. (Acromyod.) Ploceid. (Estrildin.).

Uraeotyphlus PETERS 1879 : Amph. APOD. Caeciliid.

Uragus KEYSERLING et BLASIUS 1840: Av. PASSERIF. (Acromyod.) Fringillid. (Fringillin.).

Uraleptus COSTA 1858 : P.-GADIF. Morid.

Uranichthys POEY 1867: P.-ANGUILLIF. (Congroid.) Ophichthyid.

Uranidea de KAY 1842 : P.-PERCIF. (Cottoid.) Cottid.

Uraniscodon KAUP 1826: R.-SQUAMAT. (Saur.) Iguanid.

Uranoblepus GILL 1861: P.-PERCIF. (Scorpaenoid.) Synancejid.

Uranoconger FOWLER 1934 : syn. de Congrina JORDAN et HUBBS 1925.

Uranodon ILLIGER 1811: syn. d'Hyperoodon de LACEPEDE 1804 .

Uranomitra REICHENBACH 1853 : Av. APODIF. Trochilid.

Uranomys DOLLMAN 1909 : M.RODENT. (Myomorph.) Murid. (Murin.).

Uranoscodon BONAPARTE 1832 : R.-SQUAMAT. (Saur.) Iguanid.

Uranoscopus LINNE 1758 : P.-PERCIF. (Trachinoid.) Uranoscopid.

Uraptera MULLER et HENLE 1837 : P.-RAJIF. (Rajoid.) Rajid.

Uraspis BLEEKER 1855: P.-PERCIF. (Percoid.) Carangid.

Uratelornis ROTSCHILD 1895 : Av. CORACIADIF. Coraciadid. (Coraciadin.), 
Uria MOEHRING 1758: Av. ALCIF. Alcid. (Alcin.).

Uribos HEUDE 1901: syn. de Bos LINNE 1758.

Urichthys SWAINSON 1839 : P.PERCIF. (Labroid.) Labrid.

Urile BONAPARTE 1855: syn. de Phalacrocorax BRISSON 1760.

Urinator de LACEPEDE 1799 : syn. de Colymbus LINNE 1758.

Urinophilus EIGENMANN 1918 : P.-CYPRINIF. (Siluroid.) Trichomycterid.

Uriphaëton SWAINSON 1839 : P.PERCIF. (Percoid.) Serranid.

Uroaëtus KAUP 1844: Av. FALCONIF. Falconid. (Aquilin.).

Urobatis GARMAN 1913 : P.-RAJIF. (Dasyatoid.) Dasyatid.

Urobrachya BONAPARTE 1850 : s./g. de Coliuspasser RUPPELL 1840.

Urocampus GUNTHER 1879 : P.SYNGNATHIF. Syngnathid.

Urocentron (KAUP) FITZINGER 1843 : R.-SQUAMAT. (Saur.) Iyuanid.

Urocentrus KNER 1868: P.-PERCIF. (Blennioid.) Blenniid.

Urocharis SALVADORI 1881: syn. de Melanocharis SCLATER 1858.

Urochroa GOULD 1856: Av. APODIF. Trochilid.

Urochroma BONAPARTE 1856 : Av. PSITTACIF. Psittacid.

Urocichla SHARPE 1881 : Av. PASSERIF. (Acromyod.) Troglodytid.

Urocissa CABANIS 1851: Av. PASSERIF. (Acromyod.) Corvid.

Urocitellus OBOLENSKY 1927 : syn. de Citellus OKEN 1816.

Urococcyx SHELLY 1891: Av. CUCULLIF. Cucullid.

Urocolius BONAPARTE 1854: Av. COLIIF. Coliid.

Urocolus CABANIS 1860 : vide Urocolius BONAPARTE 1854.

Uroconger KAUP 1856 : P.-ANGUILLIF. (Congroid.) Congrid.

Urocricetus SATUNIN 1902: syn. de Cricetulus MILNE-EDWARDS 1867.
Urocynchramus PREJEVALSKY 1878: Av. PASSERIF. (Acromyod.) Fringillid. (Fringillin.).

Urocyon BAIRD 1857 : M.-FISSIP. Canid. (Canin.).

Uroderma PETERS 1865 : M.-CHIROPT. (Microchiropt.) Phyllostomatid. (Stenodermin.).

Urodrepanis SHELLEY 1876: Av. PASSERIF. (Acromyod.) Drepanidid.

Urodynamis SALVADORI 1881 : syn. de Eudynamis LESSON 1828.

Urogalba BONAPARTE 1854: Av. PICIF. Galbulid.

Uroglaux MAYR 1937: Av. STRIGIF. Strigid. (Bubonin.).

Urogymnus MULLER et HENLE 1837: P.-RAJIF. (Dasyatoid.) Dasyatid.

Urolais ALEXANDER 1903:Av. PASSERIF. (Acromyod.) Sylviid.

Urolestes CABANIS 1851 : Av. PASSERIF. (Acromyod.) Laniid.

Uroleuca BONAPARTE 1850: Av. PASSERIF. (Acromyod.) Corvid.

Uroloncha CABANIS 1851: Av. PASSERIF. (Acromyod.) Ploceid. (Estrildin.).

Urolophoides LINDBERG 1930 : P.-RAJIF. (Dasyatoid.) Dasyatid.

Urolophus MULLER et HENLE 1837: P.-RAJIF. (Dasyatoid.) Dasyatid.

Urolychnus SEVERTZOV 1858: syn. de Felis LINNE 1758.

Uromacer DUMERIL et BIBRON 1853: R.-SQUAMAT. (Serpent.) Colubrid.

Uromacerina do AMARAL 1930 : R.-SQUAMAT. (Serpent.) Colubrid.

Uromanis POCOCK 1924 : syn. de Manis LINNE 1758.

Uromastix MERREM 1820: R.SQUAMAT. (Saur.) Agamid.

Uromitus BONAPARTE $1854: s . / g$. d'Hirundo LINNE 1758.

Uromyias HELLMAYR 1927: Av. PASSERIF. (Mesomyod.) Tyrannid. 
Uromys PETERS 1867: M.-RODENT. (Myomorph.) Murid. (Murin.).

Uronectes GUNTHER 1862: syn. de Lycocara GILL 1884.

Uropelia BONAPARTE 1854: Av. COLUMBIF. Columbid. (Columbin.).

Uropeltis CUVIER 1829 : R.-SQUAMAT. (Serpent.) Uropeltid.

Urophycis GILL 1864 : P.-GADIF. Gadid.

Uroplates GRAY 1825 : R.-SQUAMAT. (Saur.) Geckonid. (Uroplatin.).

Uropsila SCLATER et SALVIN 1873: Av. PASSERIF. (Acromyod.) Troglodytid.

Uropsilus MILNE-EDWARDS 1872 : M.-INSECTIV. Talpid. (Uropsilin.).

Uropterygius RUPPELL 1838 : P.ANGUILLIF. (Anguilloid.) Muraenid.

Urosaura PETERS $1870: s . / g$. de Cercosaura WAGLER 1830.

Urosaurus HALLOWELL 1854 : R.SQUAMAT. (Saur.) Iguanid.

Urosciurus ALLEN 1915: syn. de Sciurus LINNE 1758.

Urospatha SALVADORI 1869: Av. CORACIADIF. Momotid.

Urosnhena SWINHOE $1877: s . / g$. de Cettia BONAPARTE 1834.

Urospiza KAUP 1845 : Av. FALCONIF. Falconid. (Buteonin.).

Urosticte GOULD 1853: Av. APODIF. Trochilid.

Urostomias REGAN et TREWAVAS $1930: s . / g$. d'Eustomias VAILLANT 1888.

Urostrophus DUMERIL et BIBRON 1837: R. -SQUAMAT. (Saur.) Iguanid.

Urotheca BIBRON 1843 : R.-SQUAMAT. (Serpent.) Colubrid.

Urothraupis TACZANOWSKY 1885: Av. PASSERIF. (Acromyod.) Tanagrid.

Urotragus GRAY 1871: syn. de Naemorhedus SMITH 1827.

Urotrichus TEMMINCK 1841: M.INSECTIV. Talpid. (Scalopin.).

Urotriorchis SHARPE 1874: Av.
FALCONIF. Falconid. (Accipitrin.).

Urotrygon GILL 1864 : P.-RAJIF. (Dasyatoid.) Dasyatid.

Uroxis RAFINESQUE 1810 : P.-RAJIF. (Dasyatoid.) Dasyatid.

Ursarctos HEUDE 1898: syn. de Ursus LINNE 1758.

Ursitaxus HODGSON 1836 : syn. de Mellivora STORR 1780.

Ursus LINNE 1758: M.-FISSIPED. Ursid.

Urubitornis VERREAUX 1856: Av. FALCONIF. Falconid. (Buteonin.).

Urubitinga GRAY 1840: Av. FALCONIF. Falconid. (Buteonin.).

Urus SMITH 1827: syn. de Bos LINNE 1758.

Urva HODGSON 1837 : syn. de Herpestes ILLIGER 1811.

Usacaranx WHITLEY 1931: P.PERCIT. (Percoid.) Carangid.

Usinosita JORDAN et SNYDER 1900 : P.-PLEURONECTIF. (Soleoid.) Cynoglossid.

Usinosta JORDAN et SNYDER 1901 : vide Usinosita JORDAN et SNYDER 1901.

Ussuria NIKOLSKI 1904: P.-CYPRINIF. (Cyprinoid.) Cobitid.

Uta BAIRD et GIRARD 1859 : R.SQUAMAT. (Saur.) Iguanid.

\section{V}

Vacuogua JORDAN et EVERMANN 1927: P.-PERCIF. (Percoid.) Sciaenid.

Vadesuma WHITLEY 1933 : P.PERCIF. (Scorpaenoid.) Scorpaenid.

Vailima JORDAN et SALE 1906 : P.-PERCIF. (Gobioid.) Gobiid.

Vaillantella FOWLER 1905 : P.-CYPRINIF. (Cyprinoid.) Cobitid.

Vaillantia JORDAN 1878: P.-PERCIF. (Percoid.) Etheostomid.

Vaimosa JORDAN et SEALE 1906: P.-PERCIF. (Gobioid.) Gobiid.

Valamugil : P.-MUGILIF. Mugilid.

Valencia MYERS 1928: P.-PHALLOSTETHIF. Phallostethid.

Valenciennea BLEEKER 1868 : P.PERCIF. (Gobioid.) Eleotrid. 
Valenciennellus JORDAN et EVERMANN 1895 : P.-CLUPEIF. (Stomiatoid.) Sternoptychid.

Vampyressa THOMAS $1900: s . / g$. de Vampyrops PETERS 1865.

Vampyriscus THOMAS $1900: s . / g$. de Vampyrops PETERS 1865.

Vampyrodes THOMAS $1900: s . / g$. de Vampyrops PETERS 1865.

Vampyrops PETERS 1865: M.CHIROPT. (Microchiropt.) Phyllostomatid. (Stenodermin.).

Vampyrum RAFINESQUE 1815: M. - CHIROPT. (Microchiropt.) Phyllostomatid. (Phyllostomatin.).

Vandeleuria GRAY 1842: M.-RODENT. (Myomorph.) Murid. (Murin.).

Vandellia CUVIER et VALENCIENNES 1846 : P.-CYPRINIF. (Siluroid.) Trichomycterid.

Vanderbiltella FOWLER 1935 : s./g. de Barbus CUVIER et CLOQUET 1816.

Vanderhorstia SMITH 1949: P.PERCIF. (Gobioid.) Gobiid.

Vanellus MOEHRING 1758: Av. CHA R A D R I I F. Charadriid. (Charadriin.).

Vanellus BRISSON 1760 : syn. de Gavia GLOGER 1842.

Vanga VIEILLOT 1816: Av. PASSERIF. (Acromyod.) Vangid.

Vanmanenia HORA 1932: P.-CYPRINIF. (Cyprinoid.) Homalopterid.

Vansonia ROBERTS 1846 : syn. de Pipistrellus KAUP 1829.

Vanstraelenia : P.-PLEURONECTIF. (Soleoid.) Soleid.

Varanus MERREM 1820 : R.-SQUAMAT. (Saur.) Varanid.

Varicogobio LIN 1931 : syn. de Ptychidio MYERS 1930.

Varicorhinus RUPPELL 1836 : P.CYPRINIF. (Cyprinoid.) Cyprinid.

Variola SWAINSON 1839 : P.-PERCIF. (Percoid.) Serranid.

Vastres CUVIER et VALENCIENNES 1846: syn. d'Arapaima MILLER 1843.
Vauclusella WHITLEY 1931: P.PERCIF. (Blennioid.) Clinid.

Vegetichthys TANAKA 1918: P.PERCIF. (Percoid.) Lutjanid.

Velambassis WHITLEY 1935 : P.PERCIF. (Percoid.) Serranid.

Velasia GRAY 1853 : Cyclost. PETROMYZONIF. Petromyzonid.

Veles BANGS 1918: Av. CAPRIMULGIF. Caprimulgid.

Velesionymus WHITLEY 1934 : P.PERCIF. (Callionymoid.) Callionymid.

Velifer TEMMINCK et SCHLEGEL 1850: P.-LAMPRIDIF. Veliferid.

Velifracta JORDAN 1907: P.PLEURONECTIF. (Pleuronectoid.) Bothid.

Vellitor JORDAN et STARKS 1904 : P.-PERCIF. (Cottoid.) Cottid.

Venefica JORDAN et DAVIS 1892 : P.-ANGUILLIF. (Congroid.) Nettastomatid.

Veniliornis BONAPARTE $1854: A v$. PICIF. Picid.

Ventrifossa GILBERT et HUBBS 1920: P.-GADIF. Macrurid.

Veraequa JORDAN et STARKS 1904: P.-PLEURONECTIF. (Pleuronectoid.) Pleuronectid.

Verasper JORDAN et EVERMANN 1898 : P.-PLEURONECTIF. (Pleuronectoid.) Pleuronectid.

Verater JORDAN 1919 : P.-GADIF. Gadid.

Verconectes WHITLEY 1931: P.PERCIF. (Blennioid.) Clinid.

Verdithorax WHITLEY 1931: P.ANGUILLIF. (Anguilloid.) Anguillid.

Verecundum JORDAN 1890: P.PLEURONECTIF. (Pleuronectoid.) Bothid.

Verilus POEY 1860: P.-PERCIF. (Percoid.) Lutjanid.

Verma JORDAN et EVERMANN 1896 : P.-ANGUILLIF. (Anguilloid.) Echelid.

Vermivora SWAINSON 1827: Av. PASSERIF. (Acromyod.) Parulid.

Verreauxia HARTLAUB 1856 : syn. de Sasia HODGSON 1836 ou de Nannopipo CABANIS et HEINE 1863. 
Verreo JORDAN et SNYDER 1902 : P.-PERCIF. (Labroid.) Labrid.

Verriculus JORDAN et EVERMANN 1903: P. - PERCIF. (Labroid.) Labrid.

Verrugato JORDAN 1923 : P.-PERCIF. (Percoid.) Lobotid.

Verticaria COPE 1869: syn. de Cnemidophorus WAGLER 1830.

Vesicatrus EIGENMANN 1911 : P.C'YPRINIF. (Characoid.) Characid.

Vespadelus IREDALE et TROUGHTON 1934: syn. d'Eptesicus RAFINESQUE 1820.

Vesperimus COUES 1874 : syn. de Peromyscus GLOGER 1841.

Vespertilio LINNE 1758: M.-CHIROPT. (Microchiropt.) Vespertilionid. (Vespertilionin.).

Vesperugo KEYSERLING et BLASIUS 1839 : syn. de Vespertillo LINNE 1758.

Vesperus KEYSERLING et BLASIUS 1839 : syn. de Vespertillo LINNE 1758.

Vespicula JORDAN et RICHARDSON 1910 : P.-PERCIF. (Scorpaenoid.) Scorpaenid.

Vesposus JORDAN 1921 : P.-ZEIF. Zeid.

Vestiaria JAROCKI 1821 : Av. PASSERIF. (Acromyod.) Drepanidid.

Vestipedes LESSON 1843: Av. APODIF. Trochilid.

Vestula BOLIN 1946 : P.-CLUPEIF. (Myctophoid.) Myctophiid.

Veternio SNYDER 1904: P.-ANGUILLIF. (Congroid.) Congrîa.

Vetulus REICHENBACH 1862 : syn. de Macaca de LACEPEDE 1799.

Vexillicaranx FOWLER 1905 : P.PERCIF. (Percoid.) Carangid.

Vexillitrigla WHITLEY 1931: P.PERCIF. (Scorpaenoid.) Triglid.

Vibrissaphora : Amph. ANOUR. Pelobatid.

Victoriornis : Av. COLUMBIF. Didunculid.

Vidua CUVIER 1800: Av. PASSERIF. (Acromyod.) Ploceid. (Viduin.).
Vigil JORDAN 1919 : P.-PERCIF, (Nercoid.) Etheostomid.

Villarius REITTER 1896 : P.-CYPRINIF. (Siluroid.) Bagrid.

Villora HUBBS et MOTT 1935 : P.PERCIF. (Percoid.) Etheostomid.

Vimba FITZINGER 1873 : P.-CYPRINIF. (Cyprinoid.) Cyprinid.

Vinago CUVIER 1817 : Av. COLUMBIF. Columbid. (Treronin.).

Vincentia CASTELNAU 1872: P.PERCIF. (Percoid.) Serranid.

Vinciguerria JORDAN et EVERMANN 1895: P.-CLUPEIF. (Stomiatoid.) Gonostomid.

Vinculum Mc CULLOCH 1914: P. PERCIF. (Percoid.) Chaetodontid.

Vini LESSON 1833: Av. PSITTACIF. Psittacid.

Vinia SHARPE 1900: errat. pro Vini LESSON 1833.

Vipera GRONOV 1763: R.-SQUAMAT. (Serpent.) Viperid.

Virago NEWTON: Av. ANATIF. Anatid. (Anatin.).

Vireo VIEILLOT 1807 : Av. PASSERIF. (Acromyod.) Vireonid.

Vireolanius $D U B U S$ 1850: $A v$. PASSERIF. (Acromyod.) Vireonid.

Vireosa JORDAN et SNYDER 1901: P.-PERCIF. (Gobioid.) Eleotrid.

Vireosylvia BONAPARTE 1836 : Av. PASSERIF. (Acromyod.) Vireonid.

Virginia BAIRD et GIRARD 1853 : R.-SQUAMAT. (Serpent.) Colubrid.

Viridibucco OBERHOLSER 1905 : Av. PICIF. Bucconid.

Viscaccia OKEN 1816 : syn. de Lagidium MERREM 1833.

Vison GRAY 1865 : syn. de Mustela LINNE 1758.

Vitia RAMSAY 1876: Av. PASSERIF. (Acromyod.) Timaliid.

Vitraria JORDAN et EVERMANN 1903: P.-PERCIF. (Gobioid.) Gobiid.

Vitreola JORDAN et SEALE 1909 : P.-PERCIF. (Gobioid.) Gobiid. 
Viverra LINNE 1758: M.-FISSIP. Viverrid. (Viverrin.).

Viverriceps GRAY 1867: syn. de Felis LINNE 1758.

Viverricula HODGSON 1838 : M.FISSIPED. Viverrid. (Viverrin.).

Vivia HODGSON 1837: Av. PICIF. Picid. (Picin.).

Vizcacia SCHINZ 1824 : syn. de Lagostomus BROOKES 1828.

Voeltzkovia BOETTEGER 1893 : R.-SQUAMAT. (Saur.) Scincid.

Vogmarus REINHARDT 1832: errat. pro Bogmarus SCHNEIDER 1801 .

Volatinia REICHENBACH 1850 : Av. PASSERIF. (Acromyod.) Fringillid. (Pitylin.).

Volcanus GOSLINE 1954: P.-PERCIF. (Ophidioid.) Brotulid.

Volgiolus WHITLEY 1931 : s./g. de Parvicrepis WHITLEY 1931.

Volvocivora HODGSON 1837: Av. PASSERIF. (Acromyod.) Campephagid.

Vombatus GEOFFROY-ST-HILAIRE 1803 : M.-MARSUP. Vombatid.

Vomer CUVIER 1817 : P.-PERCIF. (Percoid.) Carangid.

Vomeropsis HE C K E L 1853 : P.PERCIF. (Percoid.) Carangid.

Vormela BLASIUS 1884 : M.-FISSIPED. Mustelid. (Mustelin.).

Vulpecula JAROCKI 1822: syn. d'Alopias RAFINESQUE 1810.

Vulpes OKEN 1816 : M.-FISSIP. Canid. (Canin.).

Vulpes FRISCH 1775: nom. nud. syn. de Vulpes OKEN 1816.

Vulpes FLEMING 1822: syn. de Vulpes OKEN 1816.

Vulpicanis de BLAINVILLE 1837 : syn. de Canis LINNE 1758.

Vulsiculus JORDAN et EVERMANN 1896: P.-PERCIF. (Scorpaenoid.) Triglid.

Vulsus GUNTHER 1861: P.-PERCIF. (Callionymoid.) Callionymid.

Vultur LINNE 1758: Av. FALCONIF. Vulturid.

Vultur BRISSON 1760 : syn. de Aegypius SAVIGNY 1809. w

Wagneria JENTINK 1886 : syn, de Potos GEOFFROY-ST-HILAIRE et CUVIER 1795.

Waitea JORDAN et SEALE 1906 : P.-PERCIF. (Gobioid.) Gobiid.

Waiteina FOWLER 1907: P.-CYPRINIF. (Characoid.) Characid.

Waiteopsis WHITLEY 1930 : P.PERCIF. (Gobioid.) Gobiid.

Wakiyus JORDAN et HUBBS 1925 : P.-PERCIF. (Scorpaenoid.) Platycephalid.

Walbaumina : P.-CYPRINIF. (Characoid.) Characid.

Waldronia CHAPMAN 1929: syn. de Polytmus BRISSON 1760.

Wallabia : M.-MARSUP. Macropodid. (Macropodin.).

Wallago BLEEKER 1851: P.-CYPRINIF. (Siluroid.) Silurid.

Wallagonia MYERS : syn. de Wallago BLEEKER 1851.

Wallia WERNER 1925: R.-SQUAMAT. (Serpent.) Uropeltid.

Wallsaurus UNSERWOOD 1954: R.-SQUAMAT. (Saur.) Geckonid.

Walterinnesia LATASTE 1887 : R.SQUAMAT. (Serpent.) Elapid.

Wamizichthys SMITH 1954: P.PERCIF. (Percoid.) Serranid.

Wanderer: P.-PERCIF. (Scombroid.) Scombrid.

Wangia FOWLER 1954: P.-PERCIF. (Labroid.) Pomacentrid.

Warmizichthys

Warreenula WHITLEY $1941:$ s./g. d'Astronesthes RICHARDSON 1845.

Warsanglia CLARKE 1919: Av. PASSERIF. (Acromyod.) Fringillid. (Fringillin.).

Warszewiczia BOUCARD 1895 : Av. APODIF. Trochilid.

Watasea JORDAN et SNYDER 1901 : $P$.-

Weberogobius KOUMANS 1953 : P.-PERCIF. (Gobioid.) Gobiid.

Weidomys HERSKHOWITZ 1959 : M.-RODENT. (Myomorph.) Cricetid. (Cricetin.).

Werneria POCHE 1903: Amph. ANOUR. Bufonid. 
Wernerodakaria STRAND 1928: R.-SQUAMAT. (Serpent.) Colubrid.

Wertheimeria STEINDACHNER 1877 : P.-CYPRINIF. (Siluroid.) Doradid.

Wetmorella FOWLER et BEAN 1928 : P. - PERCIF. (Labroid.) Labrid.

Wetmorena COCHERAN 1927 : R.SQUAMAT. (Saur.) Anguid.

Weyenberghia KRAGLIEVITCH 1927 : syn. de Dolichotis DESMAREST 1819.

Whitlevia (FOWLER et BEAN) CHABANAUD 1930: syn. de Paradicula WHITLEY 1931.

Whitleyidea FOWLER $1934:$ s./g. d'Alepocephalus RISSO 1820.

Whitleyina FOWLER et BEAN 1930 : syn. d'Acanthoperca CASTELNAU 1878.

Wilsonavis MATHEWS 1912 : syn. de Gerygone GOULD 1841.

Wilsonia BONAPARTE 1838: Av. PASSERIF. (Acromyod.) Parulid.

Winteria BRAUER 1902 : P.-CLUPEIF. (Opisthoproctoid.) Opisthoproctid.

Wolterstoffina: Amph. ANOUR. Leptodactylid.

Wombatula IREDALE et TROUGHTON 1934: M.-MARSUP. Vombatid.

Woodfordia NORTH 1907: Av. PASSERIF. (Acromyod.) Zosteropid.

Wuia FOWLER $1934: s . / g$. d'Heterodontus de BLAINVILLE 1816.

Wyulda ALEXANDER 1918: M.MARSUP. Phalangerid. (Phalangerin.).

\section{$\mathbf{X}$}

Xantharpyia GRAY 1843 : syn. de Rousettus GRAY 1821.

Xanthichthys RICHARDSON 1856 : P.-PERCIF. (Balistoid.) Balistid.

Xanthixus OATES 1889: syn. de Pycnonotus BOIE 1826.

Xanthocephalus BONAPARTE 1850: Av. PASSERIF. (Acromyod.) Icterid. (Agelaein.).
Xanthocorys SHARPE 1885: Av. PASSERIF. (Acromyod.) Motacillid.

Xanthogenyx d' HAMONVILLE 1883: Av. APODIF. Trochilid.

Xantholaema BONAPARTE 1854 : Av. PICIF. Capitonid.

Xanthomelus BONAPARTE 1854: Av. PASSERIF. (Acromyod.) Paradiseid. (Ptilonorhynchin.).

Xanthomixis SHARPE 1881: Av. PASSERIF. (Acromyod.) Timaliid.

Xanthomyias BERLEPSCH 1907: Av. PASSERIF. (Mesomyod.) Tyrannid.

Xanthophilus REICHENBACH 1863: Av. PASSERIF. (Acromyod.) Ploceid. (Plocein.).

Xanthoploceus $M A C K W O R T H$ PREAD et GRANT 1946: Av. PASSERIF. (Acromyod.) Ploceid. (Plocein.).

Xanthopygia BONAPARTE 1850 : Av. PASSERIF. (Acromyod.) Muscicapid.

Xanthornus PALLAS 1769: Av. PASSERIF. (Acromyod.) Icterid. (Cacicin.).

Xanthotis REICHENBACH 1852 : Av. PASSERIF. (Acromyod.) Meliphagid.

Xanthoura BONAPARTE 1850 : Av. PASSERIF. (Acromyod.) Corvid.

Xanthocles JORDAN 1917 : P.-PERCIF. (Scorpaenoid.) Hexagrammid.

Xantusia BAIRD 1858: R.-SQUAMAT. (Saur.) Xantusid.

Xema LEACH 1819: Av. LARIF. Larid. (Larin.).

Xenagama BOULENGER 1895 : R.SQUAMAT. (Saur.) Agamid.

Xenagoniates MYERS 1942 : P.-CYPRINIF. (Characoid.) Characid.

Xenambassis SCHULTZ 1945: $P$.PERCIF. (Percoid.) Serranid.

Xenanthias REGAN 1908 : P.-PERCIF. (Percoid.) Serranid.

Xenatherina REGAN 1907 : P.-MUGILIF. Atherinid.

Xenelaphis GUNTHER 1864: R.SQUAMAT. (Serpent.) Colubrid. 
Xenendum JORDAN et SNYDER 1901 : P. - CYPRINODONTIF. Goodeid.

Xenengraulis JORDAN et SEALE 1925: P.-CLUPEIF. (Clupeoid.) Engraulid.

Xenetodon REGAN 1911: P.-BELONIF. (Belonoid.) Belonid.

Xeneretmus GILBERT 1903: P.PERCIF. (Cottoid.) Agonid.

Xenerpestes BERLEPSCH 1886 : Av. PASSERIF. (Mesomyod.) Furnariid.

Xenica WESTWOOD 1851: Av. PASSERIF. (Acromyod.) Xenicid.

Xenichthys GILL 1863 : P.-PERCIF. (Percoid.) Lutjanid.

Xenicopsis CABANIS 1860: Av. PASSERIF. (Mesomyod.) Dendrocolaptid.

Xenicopsoides CORY 1919: Av. PASSERIF. (Mesomyod.) Dendrocolaptid.

Xenicornis IREDALE et MATHEWS 1926: Av. PASSERIF. (Acromyod.) Xenicid.

Xenicus GRAY 1855: Av. PASSERIF. (Acromyod.) Xenicid.

Xenisma JORDAN 1877: P.-CYPRINODONTIF. Poeciliid.

Xenistius JORDAN et GILBERT 1882: P.-PERCIF. (Percoid.) Lutjanid.

Xenobatrachus PETERS et DORIA 1878: Amph. ANOUR. Microhylid.

Xenoboa HOGE 1953: R.-SQUAMAT. (Serpent.) Boid.

Xenobuglossus : P.-PLEURONECTIF. (Soleoid.) Soleid.

Xenobythites RADCLIFFE 1913 : P.PERCIF. (Ophidioid.) Brotulid.

Xenocalamus GUNTHER 1868 : R.SQUAMAT. (Serpent.) Boigid.

Xenocara REGAN 1904 : P.-CYPRINIF. (Siluroid.) Loricariid.

Xenocephalus KAUP 1858 : P.-GADIF. Macrurid.

Xenoceratias REGAN et TREWAVAS 1932 : P.-LOPHIIF. (Ceratoid.) Melanocetid.

Xenocharax GUNTHER 1867 : P.-
CYPRINIF. (Characoid.) Characid.

Xenocheilichthys SMITH 1934 : P.CYPRINIF. (Cyprinoid.) Cyprinid.

Xenochirus GILBERT 1890 : P.PERCIF. (Cottoid.) Agonid.

Xenochromis BOULENGER 1899 : P.-PERCIF. (Percoid.) Cichlid.

Xenochropis GUNTHER 1864: R.SQUAMAT. (Serpent.) Colubrid.

Xenocichla HARTLAUB 1857 : Av. PASSERIF. (Acromyod.) Pycnonotid.

Xenoclarias GREENWOOD 1958 : P.-CYPRINIF. (Siluroid.) Clariid.

Xenoconger REGAN 1912 : P.-ANGUILLIF. (Anguilloid.) Xenocongrid.

Xenocopsoides : Av. PASSERIF. (Mesomyod.) Furnariid.

Xenocopsychus HARTERT 1907: Av. PASSERIF. (Mesomyod.) Dendrocolaptid.

Xenoctenes MILLER $1907: s . / g$. de Micronycteris GRAY 1866.

Xenoctistes HELLMAYR 1925 : syn. de Syndactyla REICHENBACH 1853.

Xenocypris GUNTHER 1808: P.CYPRINIF. (Cyprinoid.) Cyprinid.

Xenocys JORDAN et BOLLMAN 1889: P.-PERCIF. (Percoid.) Lutjanid.

Xenodacnis CABANIS 1873: Av. PASSERIF. (Acromyod.) Coerebid.

Xenodermus REINHARDT 1837: R.-SQUAMAT. (Serpent.) Colubrid.

Xenodexia HUBBS 1950 : P.-CYPRINODONTIF. Poeciliid.

Xenodermichthys GUNTHER 1870: P.-CLUPEIF. (Alepocephaloid.) Alepocephalid.

Xenodon BOIE 1826: R.-SQUA. MAT. (Serpent.) Colubrid.

Xenogale ALLEN 1919 : M.-FISSIP. Viverrid. (Herpestin.).

Xenognathus GILBERT 1915 : P.CLUPEIF. (Alepocephaloid.) Alepocephalid. 
Xenogramma WAITE 1904: P.PERCIF. (Trichiuroid.) Gempylid.

Xenojulis de BEAUFORT 1939 : P.PERCIF. (Labroid.) Labrid.

Xenolepidichthys GILCHRIST 1928 : P.-ZEIF. Zeid. (ou Grammicolepidid.).

Xenomelaniris SCHULTZ 1948 : P.MUGILIF. Atherinid.

Xenomugil SCHULTZ 1946: $P$.MUGILIF. Mugilid.

Xenomys MERRIAM 1892 : M.-RODENT. (Myomorph.) Cricetid. (Cricetin.).

Xenomystax GILBERT 1891: P.ANGUILLIF. (Congroid.) Congrid.

Xenomystus LUTKEN 1873 : P.-CYPRINIF. (Siluroid.) Callichthyid.

Xenomystus GUNTHER 1868: P.CLUPEIF. (Notopteroid.) Notopterid.

Xenonetta FLEMING 1935: Av. ANATIF. Anatid. (Anatin.).

Xenoophorus HUBBS et TURNER 1939 : P. - CYPRINODONTIF. Goodeid.

Xenopeltis REINWARDT 1826 : R.SQUAMAT. (Serpent.) Xenopeltid.

Xenophallus HUBBS 1925 : P.CYPRINODONTIF. Poeciliid.

Xenopholis PETERS 1869 : R.SQUAMAT. (Serpent.) Boigid.

Xenopholis DAVIS 1887: syn. de Xenopholoides FOWLER 1958.

Xenopholoides FOWLER 1958 : P.PERCIF. (Blennioid.) Blenniid.

Xenophrynichthys SCHULTZ 1957: s./g. d'Antennatus SCHULTZ 1957.

Xenophthalmichthys REGAN 1925: P.-CLUPEIF. (Opisthoproctoid.) Microstomid.

Xenopicus BAIRD 1858: Av. PICIF. Picid. (Picin.).

Xenopipo $C A B A N I S$ 1847: Av. PASSERIF. (Mesomyod.) Piprid.

Xenopirostris BONAPARTE 1850 : Av. PASSERIF. (Acromyod.) Vangid.
Xenopoclinus : P.-PERCIF. (Blennioid.) Clinid.

Xenopoecilus REGAN 1911 : P.CYPRINODONTIF. Adrianichthyid.

Xenopomichthys PELLEGRIN 1905: syn. de Kneria STEINDACHNER 1866.

Xenops ILLIGER 1811: Av. PASSERIF. (Mesomyod.) Dendrocolaptid.

Xenopterus (BIBRON) TROSCHEL 1856 : P.-TETRAODONTIF.

(Tetraodontoid.) Tetraodontid.

Xenopus WAGLER 1827: Amph. ANOUR. Pipadid.

Xenorhina PETERS 1863: Amph. ANOUR. Microhylid.

Xenorhynchichthys REGAN 1908 : P.-MUGILIF. Mugilid.

Xenorhynchus BONAPARTE 1855 : Av. ARDEIF. Ciconid.

Xenosaurus $P$ E T E R $S$ 1861: R.SQUAMAT. (Saur.) Xenosaurid.

Xenoscarops SCHULTZ 1958: P. PERCIF. (Labroid.) Scarid.

Xenoscarus EVERMANN et RADCLIFFE 1917 : P.-PERCIF. (Labroid.) Scarid.

Xenospiza BANGS 1931: Av. PASSERIF. (Acromyod.) Fringillid.

Xenotaenia TURNER 1946 : P.-CYPRINODONTIF, Goodeid. ou Poeciliid.

Xenotilapia BOULENGER 1899 : P.PERCIF. (Percoid.) Cichlid.

Xenotis JORDAN 1877 : P.-PERCIF. (Percoid.) Centrarchid.

Xenotoca HUBBS et TURNER 1939: P.-CYPRINODONTIF. Goodeid.

Xenotriccus DWIGHT et GRISCOM 1927: Av. PASSERIF. (Mesomyod.) Tyrannid.

Xenurobrycon MYERS et MIRANDA-RIBEIRO 1945 : P.-CYPRINIF. (Characoid.) Characid.

Xenurocharax REGAN 1913 : P.CYPRINIF. (Characoid.) Characid.

Xenurophis GUNTHER 1863 : R.SQUAMAT. (Serpent.) Colubrid.

Xenurus WAGLER 1830 : syn. de Cabassous Mc MURTRIE 1831.

Xenus KAUP 1829: Av. ARDEIF. Ardeid. 
Xeocephus BONAPARTE 1854 : Av. PASSERIF. (Acromyod.) Muscicapid.

Xeromys THOMAS 1889: M.-RODENT. (Myomorph.) Murid. (Hydromyin.).

Xerophila GOULD 1841: Av. PASSERIF. (Acromyod.) Parid.

Xerospermophilus MERRIAM 1893: s./g. de Citellus OKEN 1816.

Xerus HEMPRICH et EHRENBERG 1833: M.-RODENT. (Sciuromorph.) Sciurid. (Sciurin.).

Xesurus JORDAN et EVERMANN 1896 : P.-PERCIF. (Acanthuroid.) Acanthurid.

Xiliphius EIGENMANN 1912 : P.CYPRINIF. (Siluroid.) Bunocephalid.

Xiphasia $S$ W A IN S ON 1839 : P.PERCIF. (Blennioid.) Blenniid.

Xiphias LINNE 1758 : P.-PERCIF. (Scombroid.) Xiphiid.

Xiphichthys SWAINSON 1839 : P.LAMPRIDIF. Trachypterid.

Xiphidion GIRARD 1859: P.-PERCIF. (Blennioid.) Blenniid.

Xiphidiopicus BONAPARTE 1854 : Av. PICIF. Picid. (Picin.).

Xiphidiopterus REICHENBACH 1852: Av. CHARADRIIF. Charadriid. (Charadriin.).

Xiphypops JORDAN 1923 : P.-PERCIF. (Percoid.) Chaetodontid.

Xiphirhynchus BLYTH 1842: Av. PASSERIF. (Acromyod.) Timaliid.

Xiphister JORDAN et STARKS 1895 : P.-PERCIF. (Blennioid.) Blenniid.

Xiphius AGASSIZ 1846: emend. pro Ziphius CUVIER 1823.

Xiphocercus FITZINGER 1843 : R.SQUAMAT. (Saur.) Iguanid.

Xiphocharax FOWLER 1914 : P.CYPRINIF. (Characoid.) Characid.

Xiphocheilus BLEEKER 1856 : P.PERCIF. (Labroid.) Labrid.

Xiphocolaptes LESSON 1840: Av. PASSERIF. (Mesomyod.) Furnariid. ou Dendrocolaptid.

Xiphogadus GUNTHER 1862: P.PERCIF. (Blennioid.) Blenniid.
Xipholena GLOGER 1842 : Av. PASSERIF. (Mesomyod.) Cotingid.

Xiphonycteris DOLLMANN 1911 : M. -CHIROPT. (Microchiropt.) Molossid.

Xiphophorus HECKEL 1848 : P.CYPRINODONTIF. Poeciliid.

Xiphorhamphus MULLER et TROSCHEL 1844: P.-CYPRINIF. (Characoid.) Characid.

Xiphorhynchus AGASSIZ 1829: syn. d'Acestrorhynchus EIGENMANN 1903.

Xyphorhynchus SWAINSON 1827 : Av. PASSERIF. (Mesomyod.) Dendrocolaptid.

Xiphosoma WA GLER 1824: R.SQUAMAT. (Serpent.) Boid.

Xiphostoma SPIX 1829 : P.-CYPRINIF. (Characoid.) Characid.

Xiphotheca AGASSIZ 1846 : P.PERCIF. (Trichiuroid.) Trichiurid.

Xolmis BOIE 1828 : Av. PASSERIF. (Mesomyod.) Tyrannid. (Fluvicolin.).

Xurel JORDAN et EVERMANN 1927: P.-PERCIF. (Percoid.) Carangid.

Xylocopus CABANIS 1863: Av. PICIF. Picid. (Picin.).

Xylomys MERRIAM $1902: s . / g$. de Heteromys DESMAREST 1817.

Xylophis BEDDOME 1878 : R.SQUAMAT. (Serpent.) Colubrid.

Xypterus RAFINESQUE 1810 : P.LAMPRIDIF. Trachypetrid.

Xyrauchen KIRSCH 1889: P.-CYPRINIF. (Cyprinoid.) Catostomid.

Xyrias JORDAN et SNYDER 1901 : P.ANGUILLIF. (Congroid.) Ophichthyid.

Xyrichthys QUOY et GAIMARD 1824: P.-PERCIF. (Labroid.) Labrid.

Xyrocharax FOWLER 1914 : P.-CYPRINIF. (Characoid.) Characid.

Xyrula JORDAN 1891 : P.-PERCIF. (Labroid.) Labrid.

Xystaema JORDAN 1895 : P.-PERCIF. (Percoid.) Leiognathid.

Xyster de LACEPEDE 1803: P.PERCIF. (Percoid.) Kyphosid. 
Xysterus RAFINESQUE 1815 : P.PERCIF. (Percoid.) Kyphosid.

Xystes JORDAN et STARKS 1895 : P.-PERCIF. (Cottoid.) Agonid.

Xystodus OGILBY 1911 : P.-CLUPEIF. (Myctophoid.) Synodid.

Xystramia JORDAN 1917 : P.-PERCIF. (Percoid.) Serranid.

Xystreurys JORDAN 1881: P.PLEURONECTIF, (Pleuronectoid.) Bothid.

Xystrias JORDAN et STARKS 1904: P.-PLEURONECTIF. (Pleuronectoid.) Pleuronectid.

Xystroperca JORDAN et EVERMANN 1896 : P.-PERCIF. (Percoid.) Serranid.

Xystrophorus RICHARDSON 1845 : P.-PERCIF. (Percoid.) Carangid.

Xystronlites JORDAN 1877 : P.PERCIF. (Percoid.) Centrarchid.

Xystrosus JORDAN et SNYDER 1901: P.-CYPRINIF. (Cyprinoid.) Cyprinid.

Yabotichthys HERRE 1945: P.PERCIF. (Gobioid.) Gobiid.

Yaci de BUEN : P.-MUGILIF. Atherinid.

Yanacea CARRIKER 1933: Av. PASSERIF. (Mesomyod.) Tyrannid. (Spizithornin.).

Yaoshanicus LIN 1931 : P.-CYPRINIF. (Cyprinoid.) Cyprinid.

Yarica WHITLEY 1930: P.-PERCIF. (Percoid.) Serranid.

Yarra CASTELNAU 1872 : Cyclost. PETROMYZONIF. Petromyzonid.

Yarella GOODE et BEAN 1895 : P.CLUPEIF. (Stomiatoid.) Gonostomid.

Yarutius WHITLEY 1931 : P.-PERCIF. (Callionymoid.) Callionymid.

Yirrkala WHITLEY 1940 : P.-ANGUILLIF. (Congroid.) Ophichthyid.

Yoga WHITLEY $1954:$ s./g. de Ctenogobius GILL 1858.
Yongeichthys WHITLEY 1932 : P.PERCIF. (Gobioid.) Gobiid.

Yozia JORDAN et SNYDER 1901: P. -SYNGNATHIF. Solenostomid.

Ypsigramma SCHULTZ 1953 : P.PERCIF. (Percoid.) Serranid.

Ypsiscarus SCHULTZ 1958: $s . / g$. de Scarus GRONOV 1763.

Yuhina HODGSON 1837: Av. PASSERIF. (Acromyod.) Timaliid.

Yungipicus BONAPARTE 1854: Av. PICIF. Picid. (Picin.).

Yunx LINNE 1766: syn. de Jynx LINNE 1758.

Yuriria JORDAN et EVERMANN 1896 : P.CYPRINIF. (Cyprinoid.) Cyprinid.

\section{$\mathbf{z}$}

Zabidius WHITLEY 1930 : P.-PERCIF. (Percoid.) Ephippid.

Zabulon WHITLEY 1928 : P.-PERCIF. (Labroid.) Pomacentrid.

Zacco JORDAN et EVERMANN 1902: P.-CYPRINIF. (Cyprinoid.) Cyprinid.

Zachaenus COPE 1866: Amph. ANOUR. Leptodactylid.

Zaclemus GILBERT 1896 : P.-PERCIF. (Percoid.) Sciaenid.

Zaëdypus AMEHINGO 1889 : M.EDENT. (Xenarthr.) Dasypodid. (Euphractin.).

Zaglossus GILL 1877 : M.-MONOTREM. Tachyglossid.

Zalanthias JORDAN et RICHARDSON 1910 : P.-PERCIF. (Percoid.) Serranid.

Zalarges JORDAN et WILLIAMS 1895: P.-CLUPEIF. (Stomiatoid.) Gonostomid.

Zalembius JORDAN et EVERMANN 1896: P.-PERCIF. (Labroid.) Embiotocid.

Zalescopus JORDAN et $H U B B S$ 1925: P.-PERCIF. (Trachinoid.) Uranoscopid.

Zalieutes JORDAN et EVERMANN 1896 : P.-LOPHIIF. (Ogcocephaloid.) Ogcocephalid.

Zalistes JORDAN et SNYDER 1901 : P.-PERCIF. (Pegasoid.) Pegasid. 
Zalocys JORDAN et Mc GREGOR 1899 : P.-PERCIF. (Percoid.) Carangid.

Zalophus GILL 1866: M.-PINNIP. Otariid. (Otariin.).

Zalopyr : s./g. de Sebastodes GILL 1861.

Zalypnus JORDAN et EVERMANN 1896 : P.-PERCIF. (Gobioid.) Gobiid.

Zamelodia COUES 1880 : Av. PASSERIF. (Acromyod.) Fringillid.

Zamenis WAGLER 1830 : R.-SQUAMAT. (Serpent.) Colubrid.

Zameus JORDAN et FOWLER 1903 : P.-SQUALIF. (Squalioid.) Squalid.

Zamora WHITLEY 1931 : syn. de Bessetina WHITLEY 1941.

Zanclistius JORDAN 1907 : P.-PERCIF. (Percoid.) Lutjanid.

Zanclorhynchus GUNTHER 1880 : P.PERCIF. (Percoid.) Chaetodontid.

Zanclostomus SWAINSON 1837: Av. CUCULLIF. Cucullid.

Zanclurus SWAINSON. 1839 : P.PERCIF. (Scombroid.) Istiophorid.

Zanclus (Commerson) CUVIER et VALENCIENNES 1831 : P.PERCIF. (Acanthuroid.) Zanclid.

Zaniolepis GIRARD 1857 : P.-PERCIF. (Ophidioid.) Ophidiid.

Zantecla CASTELNAU 1873 : P.MUGILIF. Atherinid.

Zanthomiza SWAINSON 1837: Av. PASSERIF. (Acromyod.) Meliphagid.

Zanthopygia BLYTH 1847 : Av. PASSERIF. (Acromyod.) Muscicapid.

Zaocys COPE 1860 : R.-SQUAMAT. (Serpent.) Colubrid.

Zaphotias GOODE et BEAN 1898 : syn. de Bonapartia GOODE et $B E A N 1896$.

Zapornia LEACH 1816: Av. RALLIF. Rallid.

Zaprora JORDAN 1896 : P.-PERCIF. (Trachinoid.) Pinguipedid.

Zapteryx JORDAN 1881: syn. de Syrrhina MULLER et HENLE 1841.
Zapus COUES 1876 : M.-RODENT. (Myomorph.) Dipodid. (Zapodin.).

Zarhynchus OBERHOLSER 1899 : Av. PASSERIF. (Acromyod.) Icterid.

Zastomias GILBERT 1915 : P.-CLUPEIF. (Stomiatoid.) Stomiatid.

Zathorax COPE 1872: P.-CYPRINIF. (Siluroid.) Doradid.

Zati REICHENBACH 1862 : syn. de Macaca de LACEPEDE 1799.

Zavattariornis MOLTONI 1938: Av PASSERIF. (Acromyod.) Corvid. (Zavattariornithin.).

Zeablennius WHITLEY 1930 : P.PERCIF. (Blennioid.) Blenniid.

Zeanida : vide Zenaida BONAPARTE 1838.

Zearaja WHITLEY 1939 : P.-RAJIF. (Rajoid.) Rajid.

Zebrapicus MALHERBE 1849: Av. PlCIF. Picid. (Picin.).

Zebrasoma SWAINSON 1839 : P.PERCIF. (Acanthuroid.) Acanthurid.

Zebreleotris HERRE 1953 : P.-PERCIF. (Gobioid.) Eleotrid.

Zebrias JORDAN et SNYDER 1900 : P.-PLEURONECTIF. (Soleoid.) Soleid.

Zebrilus BONAPARTE 1855: Av. ARDEIF. Ardeid.

Zebrus de BUEN 1930: s./g. de Gobius LINNE 1758.

Zecoturnix IREDALE et MATHEWS 1926: Av. PHASIANIF. Phasianid. (Phasianin.).

Zelotomys OSGOOD 1910 : M.-RODENT. (Myomorph.) Murid. (Murin.).

Zeluco WHITLEY 1931: P.-PERCIF. (Percoid.) Sciaenid.

Zen JORDAN 1903 : P.-ZEIF. Zeid,

Zenaida BONAPARTE 1838: Av. COLUMBIF. Columbid. (Columbin.).

Zenaidura BONAPARTE 1854:Av. COLUMBIF. Colnmbid. (Columbin.).

Zenarchopterus GILL 1864 : P.-BELONIF. (Exocoetoid.) Hemirhamphid.

Zenion JORDAN et EVERMANN 1896 : P.-ZEIF. Zeid. 
Zenkerella MATSCHIE 1898: M.RODENT. (Theridomyomorph.) Anomalurid. (Zenkerellin.).

Zenoida des MURS 1856 : syn. de Zenaidura BONAPARTE 1854

Zenopsis GILL 1862 : P.-ZEIF. Zeid.

Zeocephus SHARPE 1879: vide Xeocephus BONAPARTE 1854.

Zeodrius CASTELNAU 1879: P. PERCIF. (Percoid.) Cirrhitid.

Zesticelus JORDAN et EVERMANN 1896 : P.-PERCIF. (Cottoid.) Cottid.

Zestichthys JORDAN et HUBBS 1925: P.-PERCIF. (Blennioid.) Zoarcid.

Zestidium JORDAN et EVERMANN 1898: P.-PERCIF. (Percoid.) Sciaenid.

Zestis JORDAN et EVERMANN 1898 : P.-PERCIF. (Percoid.) Sciaenid.

Zetis THOMAS 1908 : syn. de Dremomys HEUDE 1898.

Zeugopterus GOTTSCHE 1835 : P.PLEURONECTIF. (Pleuronectoid.) Bothid.

Zeus LINNE 1758: P.-ZEIF. Zeid.

Zev WHITLEY 1927: P.-GALEIF. (Isuroid.) Orectolobid.

Zevaia CHABANAUD 1943 : P.PLEURONECTIF. (Soleoid.) Soleid.

Zezera JORDAN et FOWLER 1903 : P.-CYPRINIF. (Cyprinoid.) Cyprinid.

Zibellina KAUP 1829 : syn. de Martes PINEL 1792.

Zibethailurus SEVERTZOV 1858: syn. de Felis LINNE 1758.

Zingel OKEN 1817 : P.-PERCIF. (Percoid.) Percid.

Ziphiorhynchus BURMEISTER 1865: syn. de Ziphius CUVIER 1823.

Ziphister: vide Xiphister.

Ziphius CUVIER 1823: M.-CETAC. (Odontocet.) Ziphiid.

Zoarces CUVIER et VALENCIENNES 1836 : P.-PERCIF. (Blennioid.) Zoarcid.

Zoarchias JORDAN et $S N Y D E R$ 1902: P.-PERCIF. (Blennioid.) Zoarcid.
Zoarcites ZUGMAYER 1914：P. PERCIF. (Blennioid.) Zoarcid.

Zodalia MULSANT 1874: Av. APODIF. Trochilid.

Zokor ELLERMAN 1941: syn. d'Eospalax ALLEN 1938.

Zonaeginthus CABANIS 1851: Av. PASSERIF. (Acromyod.) Ploceid. (Estrildin.).

Zonibyx REICHENBACH 1852: Av. CHARADRIIF, Charadriid. (Charadriin.).

Zonichthys SWAINSON 1839: P. PERCIF. (Percoid.) Carangid.

Zonifer SHARPE 1896: Av. CHARADRIF. Charadriid. (Charadriin.).

Zonobatus GARMAN : syn. de Platyrhinoides JORDAN 1881.

Zonogobius BLEEKER 1874: syn. de Priolepis CUVIER et VALENCIENNES 1837.

Zonophichthys WHITLEY 1930 : P.-ANGUILLIF. (Congroid.) Ophichthyid.

Zonosaurus BOULENGER 1887 : R.-SQUAMAT. (Saur.) Gerrhosaurid.

Zonoscion JORDAN et EVERMANN 1896: P.-PERCIF. (Percoid.) Sciaenid.

Zonotrichia SWAINSON 1832: Av. PASSERIF. (Acromyod.) Fringillid. (Emberizin.).

Zonurus MERREM 1820 : R.-SQUAMAT. (Saur.) Zonurid.

Zoogoneticus MEEK 1902: P.-CYPRINODONTIF. Goodeid.

Zoonavena MATHEWS 1918: Av. APODIF. Apodid.

Zoothera VIGORS 1832: Av. PASSERIF. (Acromyod.) Turdid.

Zootoca WAGLER 1830: s./g. de Lacerta LINNE 1758.

Zopa FITZINGER 1873 : P.-CYPRINIF. (Cyprinoid.) Cyprinid.

Zophendum JORDAN 1878 : P.-CY PRINIF. (Cyprinoid.) Cyprinid.

Zoramia JORDAN 1915: P.-PERCIF. (Percoid.) Serranid.

Zorilla OKEN 1816: M.-FISSIP. Mustelid. (Mustelin.).

Zostericola ILJIN 1927: syn. de Zosterisessor WHITLEY 1935. 
Zosterisessor WHITLEY 1935 : P.PERCIF. (Gobioid.) Gobiid.

Zosterops VIGORS et HORSFIELD 1826: Av. PASSERIF. (ACTOmyod.) Zosteropid.

Zosterornis OGILVIE-GRANT 1894: Av. PASSERIF. (Acromyod.) Timaliid.

Zunasia JORDAN et METZ 1913 : P.-CLUPEIF. (Clupeoid.) Clupeid.

Zungaro BLEEKER 1858 : P.-CYPRINIF. (Siluroid.) Bagrid.

Zungaropsis STEINDACHNER 1908: P.-CYPRINIF. (Siluroid.) Bagrid.

Zygaena CUVIER 1817: syn. de Sphyrna RAFINESQUE 1810.

Zygaspis COPE 1885: R.-SQUAMAT. (Serpent.) Amphisbaenid.
Zygobatis AGASSIZ 1838: P.-RAJIF. (Dasyatoid.) Myliobatid.

Zygodontomys ALLEN 1897 : M.RODENT. (Myomorph.) Cricetid. (Cricetin.).

Zygogaster EIGENMANN 1913 : P.CYPRINIF. (Characoid.) Characid.

Zygogeomys MERRIAM 1895: M.RODENT. (Myomorph.) Geomyid.

Zygonectes AGASSIZ 1854 : P.-CYPRINODONTIF. Cyprinodontid.

Zyphothyca SWAINSON 1839 : P.PERCIF. (Trichiuroid.) Trichiurid.

Zyzomys THOMAS 1909: M.-RODENT. (Myomorph.) Murid. (Murin.). 


\section{TABLE DES AUTEURS (1)}

A

Amyot (Bernard). - v. Condoret (A.), Bailenger (J.) et -

Arfan (Fayedoum). - v. Gaud (J.), - et Zeini (A.).

ArfaA (Fereydun). - A study on Paramphistomum microbothrium in Khuzis-

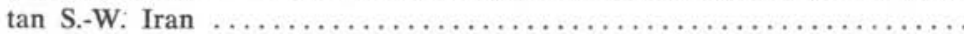

Bailenger (Jean) et LaGarde (E.). - Teigne favique du cuir chevelu de l'enfant déterminée par Sabouraudites gypseus (Bodin 1907) Ota et Langeron

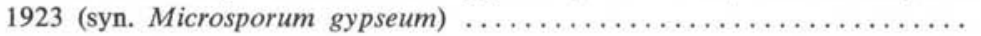

-. v. Condoret (A.), - et Amyot (B.).

Barbosa (Frederico S.). - Problèmes de nomenclature au sujet des vecteurs actuels et potentiels de Schistosoma mansoni en Afrique et en Amérique . .

Beaucournu (J.-C.). - Nouvelles captures de Nycteribiidae (Diptera, Pupi-

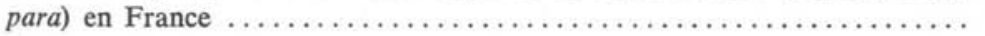

Bhatia (B.-B). - v. Pande (B.-P.), Rai (P.) and -

Biguet (Jean), Capron (A.) et Tran-Van Ky (P.). - Les antigènes de Fasciola

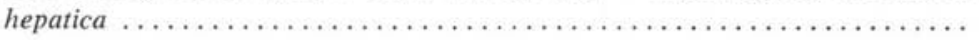

Brygoo (Edouard-R.). - v. Chabaud (A.-G.) et -, v. Deblock (S.), Capron (A.) et -, v. Grétillat (S.), - et Domergue (C.-A.).

Buttner (Alice). - v. Rousset (J.-J.), Houin (R.) et -

\section{C}

CAllot (J.). - Nouvelle localisation continentale française d'Aedes vittatus

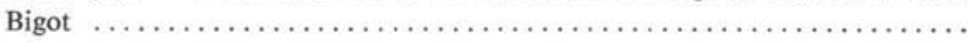

-, Krémer (M.) et Deduit (Y.). - Nouvelles espèces et nouvelles localisations de Culicoides (Diptera: Ceratopogonidae) des Ardennes, du Centre de la France, du Jura Français et du Jura suisse ...................

Capron (André). - v. Biguet (J.), - et Tran-Van Ky (P.), v. Deblock (S.) et -, v. Deblock (S.), - et BRygoo (E.-R.).

(1) Chiffres romains: articles originaux.

Chiffres italiques: revues critiques, notes et informations, analyses, répertoires. 
Chabaud (Alain-G.) et Brygoo (E.-R.). - Nématodes parasites de Caméléons

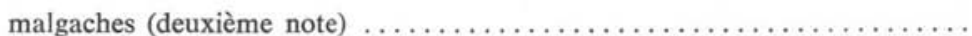

-. v. Mazzoti (L.) et -

Chevrel (Marie-Louise). - v. Doby-Dubois (M.), - , Doby (J.-M.) et RoBERT (Y.).

Condoret (Ange), Bailenger (J.) et Amyot (B.). - La piroplasmose canine dans le Sud-Ouest de la France. I. Epidémiologie ..................

Coste (Marguerite). - v. Galliard (H.), Lapierre (J.) et -

Coumbaras (Alexis). - Devenir et avenir de la bilharziose en Tunisie ....

\section{D}

Deblock (Stéphane) et CAPron (A.). - Description d'Astriotrema (Biguetrema) tananarivensis, n. subgen., n. sp., Plagiorchiidae intestinal d'Amphi-

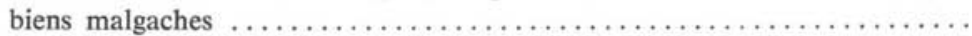

-, Capron (A.) et Brygoo (E.-R.). - Trématodes de Caméléons de Madagascar. II. Description de trois espèces nouvelles des genres Lecithoden-

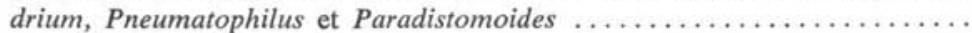

- et Dinouré (A.). - Quelle est la valeur du genre Thysanotaenia Beddard, 1911 ? (A propos d'une redescription de $T$. lemuris Beddard, Anoplocepha-

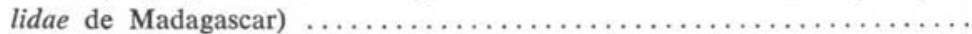

- et Rose (F.). - Les Hymenolepis (sensu lato) de Charadriiformes. (A pro-

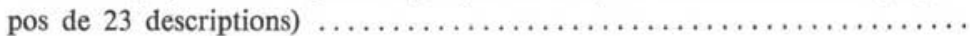

Deduit (Y.). - v. Callot (J.), Krémer (M.) et -

Deltour (Françoise), - v. Rioux (J.-A.), Golvan (Y.-J.), Houin (R.) et -

De Puytorac (Pierre) et Lom (J.). - La tomitogénèse des Collinia, Ciliés apostomes sanguicoles, endoparasites des Crustacés

Diaouré (A.). - v. Deblock (S.) et -

Di Campo (A.). - v. Nicoli (R.-M.), - et Quilici (M.).

Doby (Jean-Marie). - v. Doby-Dubois (M.), Chevrel (M.-L.), - et RoBERT (Y.).

Doby-Dubois (Michèle), Chevrel (M.-L.), Doby (J.-M.) et Robert (Y.). Abcès mycosique cérébral humain par Cladosporium trichoides ..........

Dollfus (Robert-Ph.). - Variations intra-spécifiques chez un Carmyerius (Trematoda, Gastrothylacidae), parasite du buffle au Congo belge .........

—. Deux espèces de Trématodes monogénétiques parasites du \& Bluefin tuna » de Californie

Domergue (Charles-A.). - v. Grétillat (S.), Brygoo (E.-R.) et -

$\mathbf{E}$

ECHARD (Geneviève). - v. THÉOdoridès (J.) et -

Euzet (Louis) et TriLles (J.-P.). - Encore un Monogène nouveau, parasite de Peristhedion cataphractum L. (Teleostei, Triglidae) ............. 216

-. v. Prost (M.) et - 
Galliard (Henri). - Recherches sur la biologie des Culicidés à Hanoï (Tonkin, Nord-Vietnam). II. Reproduction et ponte d'Aedes albopictus,

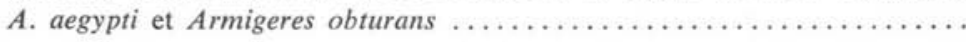

- -, Lapierre (J.) et Coste (M.). - Contribution à l'étude d'une souche

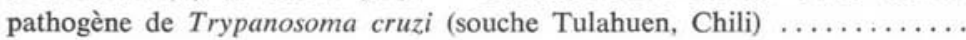

Gaud (Jean), Arfan (F.) et Zeini (A.). - Observations sur la biologie de Buli-

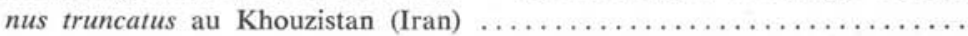

GHerman (I.). - Dégénérescence spontanée des œufs d'Hymenolepis nana, forme de manifestation de l'immunité dans cette parasitose $\ldots . \ldots \ldots \ldots$.

Giudicelli (J.) et Nicoli (R.-M.). - Contribution à l'étude du peuplement de la Corse ( $10^{\circ}$ note). Un Gordionus nouveau, Gordionus cyrnensis n. sp. (Gordioïdea)

Golvan (Yves-J.). - Le Phylum des Acanthocephala (4 ${ }^{\circ}$ note). La classe des

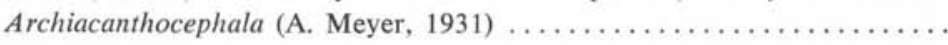

—. Répertoire systématique des noms de genres de Vertébrés 180, 419, 688,

—. Catalogue systématique des genres de Poissons actuels décrits de 1758 à 1960 ( $\mathrm{n}^{\circ} 6$ bis).

-. v. Rioux (J.-A.), - Houin (R.) et Deltour (F.).

Gordon (R.-M.) et LAVoipierRe (M.-M.-J.). - Entomology for students of Medicine. Blackwell Scientific Publications, Oxford, 1962, 353 pp. (Analyse par H. Galliard) . . . . . . . . . . . . . . . . . . . . . . . . . . .

Grassé (P.-P.), Poisson (R.) et Tuzet (O.). - Zoologie. I. Invertébrés. Précis de sciences zoologiques, Masson, édit., Paris, 1961, 919 pp. (Analyse par

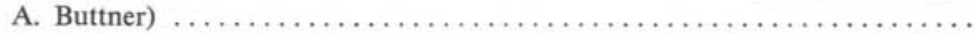

GrétIllat (Simon). - Carmyerius papillatus n. sp. et Carmyerius parvipapillatus n. sp. (Trematoda, Gastrothylacidae), parasites des réservoirs gastri-

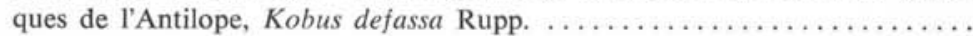

- Etude du cycle évolutif du Schistosome des Ruminants domestiques de l'Ouest africain et confirmation de l'espèce Schistosoma curassoni Brumpt,

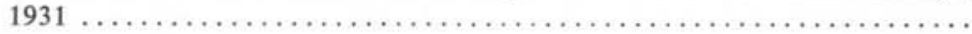

—, Brygoo (E.-R.) et Domergue (A.). - Pentastomes de Reptiles malgaches GuY (Y.). - Renseignements fournis par l'étude du rapport larves/adultes

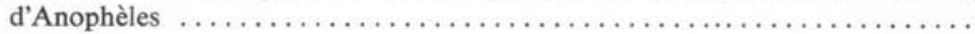

Hörning (Bernd) et Hörning-Pezendiurg (E.). - L'adiaspiromycose (haplomycose) chez les Mammifères de la Suisse $\ldots \ldots \ldots \ldots \ldots \ldots \ldots \ldots \ldots$

Hörning-Pezenburg (Erika). - v. Hörning (B.) et -

Houin (René). - v. Rioux (J.-A.), Golvan (Y.-J.), - et Deltour (F.), v. Rousset (J.-J.), - et Buttner (A.). 
$\mathbf{K}$

Krémer (M.). - v. Callot (J.), - et Deduit (Y.).

LAGARde (E.). - v. BAILenger (J.) et -

LAHOURCADE (Michel). - Quelques précisions sur la morphologie et la biologie de Scleroderma domestica Latr., petit Hyménoptère Béthylide vulnérant

LAPEYSSONIE (L.). - Eléments d'hygiène et de santé publique sous les Tropiques, Paris, 1961, Gauthier-Villars, édit., 363 pp. (Analyse par A. Buttner) Lapierre (Jacques). - v. Galliard (H.), - et Coste (M.).

LAVoIPIERRe (M.-M.-J.). - v. Gordon (R.-M.) et -.

L.F Guyon (R.). - Précis de Bactériologie, Paris, 1961, G. Doin, édit., 955 pp.

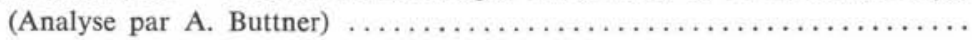

Lom (Jiri). - v. DE Puytorac (P.) et -

M

Matricon-Gondran (Michelle). - v. Nouveau (A.) et -

MAY (Jacques-M.). - Studies on Disease Ecology. Edited by -, M.D. Director, Medical Geography department, the American Geographical Society, New-York, and 13 contributors, Hafner Publ. Cy, New-York, 1961. (Ana-

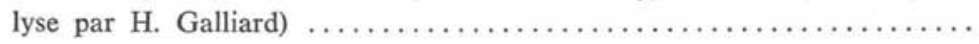

Mazzotti (Luis) et Chabaud (A.-G.). - Présence de Dipetalonema recondi-

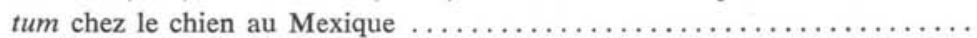

Monroe (Lee-S.). - v. Spencer (F.-M.) et -

$\mathbf{N}$

Nicoli (R.-M.), di Campo (A.) et Quilici (M.). - Une méthode simple pour l'obtention de préparations microscopiques définitives en coprologie ...... 675

-. v. Giudicelli (J.) et -

Nouveau (André) et Matricon-Gondran (M.). - Contribution à l'étude de Xiphidocercaria polyxena E. Brumpt, 1944 (Trématode), parasite de Pla-

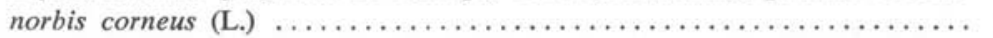

PAnde (B.-P.), RAi (P.) and Bhatia (B.-B.). - Note on a filarial infection in

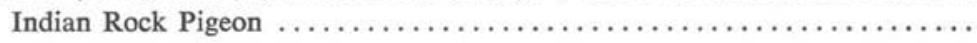

PETTER (Annie-J.). - Redescription et analyse critique de quelques espèces d'oxyures de la Tortue grecque (Testudo graeca L.). Diversité des structures

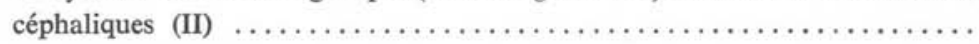

—. Synonymie de Grassenema procaviae Petter 1959, Cobboldina longicauda Köhler et Supperer 1960, et Acanthostephanocephalus caballeroi Kreis 1960 
PICK (F.). - Sur la mise en évidence expérimentale du jeu des stylets des Réduvidés hématophages, permettant la reconstitution du mécanisme de

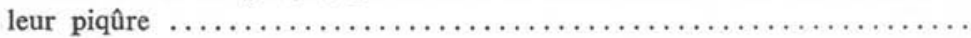

- Sur le mode de déposition des œufs par Triatoma megista et par Rhod-

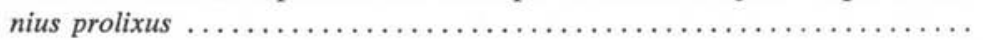

- A propos de la détermination du premier vecteur de Trypanosoma cruzi

-. Enregistrement expérimental de la démarche des Réduvidés hématophages

- Sur la signification fonctionnelle de l'ornementation des œufs des Rédu-

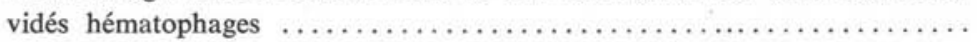

Poisson (R.). - v. Grassé (P.-P.), - et Tuzet (O.).

Prost (Maria) et Euzet (L.). - Flexophora ophidii n. g., n. sp., un Diclidophoridae (Monogenea), parasite d'Ophidium barbatum (L.) (Teleostei) ...

\section{$\mathbf{Q}$}

Quilici (M.). - v. Nicoli (R.-M.), di CAMPo (A.) et -.

\section{$\mathbf{R}$}

RAI (P.). - v. PANDE (B.-P.), - and Bhatia (B.-B.).

Raynaud (Jean-Pierre). - Splénectomie des Bovins et parasites sanguins ...

RiBeIRo (Henrique). - Un nouveau milieu de montage pour microscopie avec

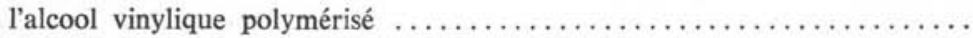

- et SoARès de Fontès E SousA (C.). - Données nouvelles sur l'élevage de Phlebotomus perniciosus Newstead 1911 (Diptera, Psychodidae) au labora-

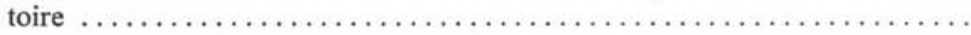

Rioux (Jean-A.), Golvan (Y.-J.), Houin (R.) et Deltour (F.). - Nouvelles stations françaises de Phlebotomus ariasi Tonnoir 1921, P. perniciosus Newstead 1911, P. mascitti Grassi 1908 et P. minutus Rondani 1843 . . . . .

Robert (Yvon). - v. Doby-Dubots (M.), Chevrel (M.-L.), Doby (J.-M.) et -

Rose (Francis). - v. Deblock (S.) et -

Rousset (Jean-Jacques), Hourn (R.) et ButTNER (A.). - Acido-alcoolo-résistance de divers œufs de Schistosomes. Modification de la technique de

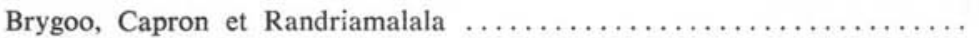

SonRès de Fontès E Sousa (Carlos). - v. Ribeiro (H.) et -

SPENCER (Francis-M.) et MonRoe (L.-S.). - The colour atlas of intestinal parasites, Charles-C. Thomas, édit., Springfield, Illinois (U.S.A.), 142 pp. (Ana-

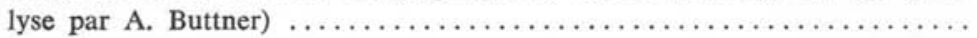

STEFANSKI (Witold). - Quelles conditions exige le parasite pour s'établir dans

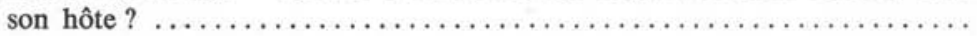


$\mathbf{T}$

THÉodoridès (Jean) et ECHARD (G.). - Stenophora gryllodes-sigillatae Narain 1961 est une Leidyana (Eugregarina, Gregarinidae) ............... 391

Tran-VAn Ky (Philippe). - v. Biguet (J.), Capron (A.) et -

Trilles (Jean-Paul). - v. EuZet (L.) et -

Tuzet (O.). - v. Grassé (P.-P.), Poisson (R.) et -

\section{$\mathbf{W}$}

WierzBicki (Krystyn). - Présence du Nématode Sterliadochona tenuissima

(Zeder 1800) dans le Nord de la Pologne

$\mathbf{z}$

Zeini (Ahmad). - v. Gaud (J.), ArfaA (F.) et - 